\title{
Aerodynamische Wirkung schnell bewegter bodennaher Körper auf ruhende Objekte
}

\author{
Dissertation
}

zur Erlangung des mathematisch-naturwissenschaftlichen Doktorgrades "Doctor rerum naturalium"

der Georg-August-Universität Göttingen

im Promotionsprogramm ProPhys

der Georg-August University School of Science (GAUSS)

vorgelegt von

Sabrina Rutschmann

aus Göttingen

Göttingen, 2017 
Betreuungsausschuss

Prof. Dr. Dr. Andreas Dillmann

PD Dr. Klaus Ehrenfried

\section{Mitglieder der Prüfungskommission:}

Referent: $\quad$ Prof. Dr. Dr. Andreas Dillmann

Institut für Aerodynamik und Strömungstechnik,

Deutsches Zentrum für Luft- und Raumfahrt e.V.

Korreferent: Prof. Dr. Wolfgang Glatzel

Institut für Astrophysik, Georg-August-Universität Göttingen

Weitere Mitglieder der Prüfungskommission:

Prof. Dr. Martin Rein

Institut für Aerodynamik und Strömungstechnik, Deutsches Zentrum für Luft- und Raumfahrt e.V.

PD Dr. Jörn Große-Knetter

II. Physikalisches Institut, Georg-August-Universität Göttingen

Prof. Dr. Ulrich Parlitz

Biomedical Physics Group, Max-Planck-Institut für Dynamik und Selbstorganisation

Prof. Dr. Andreas Tilgner

Institut für Geophysik, Georg-August-Universität Göttingen

Tag der mündlichen Prüfung: 
Man hat den Eindruck, dass die moderne Physik auf Annahmen beruht, die irgendwie dem Lächeln einer Katze gleichen, die gar nicht da ist.

(Albert Einstein) 



\section{Danksagung}

Mein ganz besonderer Dank gilt an dieser Stelle meinem Doktorvater Prof. Dr. Dr. Andreas Dillmann für die Möglichkeit, nach der Bachlor- und der Masterarbeit auch meine Dissertation am Deutschen Zentrum für Luft- und Raumfahrt anzufertigen. Er hatte immer ein offenes Ohr für meine Sorgen und Nöte und hat mich auch in schwierigen Zeiten immer unterstützt und an mich geglaubt. Diese Unterstützung hat mich auch in scheinbar ausweglosen Situationen nicht aufgeben lassen.

Vielen Dank an Prof. Dr. Claus Wagner, dass ich die Arbeit in der Abteilung Fluidsysteme in der Gruppe Verkehr durchführen durfte. Für die fachliche Betreuung bedanke ich mich bei Dr. Klaus Ehrenfried.

Ein besonderer Dank gilt Stefan Huntgeburth, der mir bei den Experimenten immer mit Rat und Tat zur Seite stand und immer ein offenes Ohr und eine helfende Hand für mich war. Danke für die guten Gespräche und fürs Zuhören!

Ich bedanke mich bei Dr. Jan Martinez Schramm, der ebenfalls immer ein offenes Ohr hatte und vielen Dank für die tollen und motivierenden Gespräche.

Ich danke Dr. Jan Martinez Schramm, Dr. Lars Graber, Stephan Hock und Jennifer Helmholdt für das Korrekturlesen und die hilfreichen Kommentare.

Ich danke meinen Freunden und meiner Familie, die mich während der gesamten Zeit unterstützt und aufgebaut haben, allen voran meiner Mutter, die mir das Physikstudium überhaupt erst ermöglichen konnte und immer für mich da ist. 



\section{Aerodynamische Wirkung schnell bewegter bodennaher Körper auf ruhende Objekte}

\section{Kurzfassung}

In einem instationären Strömungsfeld üben Druckschwankungen aerodynamische Kräfte auf Körper, wie Kugeln oder Scheiben aus. In reibungsfreien Strömungen können diese Kräfte potentialtheoretisch berechnet werden. Im instationären Strömungsfeld eines vorbeifahrenden Zuges können diese Kräfte wartende Personen am Bahnsteig oder Gleisarbeiter auf offener Strecke aus dem Gleichgewicht bringen. Bisherige Veröffentlichungen berücksichtigen für die Vorhersage der Kräfte jedoch weder die Zuggeometrie noch die Form des Körpers.

In dieser Arbeit wird ein genaueres und flexibleres analytisches Modell hergeleitet, welches es ermöglicht, die wirkenden Kräfte auf Objekte sowohl im skalierten Modellexperiment als auch im Originalmaßstab adäquat vorherzusagen.

Zur Validierung des theoretischen Modells werden skalierte Modellexperimente an der Tunnel-Simulations-Anlage Göttingen (TSG) durchgeführt. Zur genaueren Untersuchung der physikalischen Effekte der Kräfte in instationären Strömungen auf Kugeln werden anschließend Kraftmessungen im instationären Strömungsfeld eines Lautsprechers durchgeführt. Hierbei wird die Gültigkeit der Gleichung von Basset, Boussinesq und Oseen mit den Anpassungen von Odar und Hamilton überprüft, die bisher nur für kleine Reynoldszahlen bis $R e=100$ untersucht wurde.

In dieser Arbeit wird ein bisher nicht gemessener Bereich von $R e=200$ bis $R e=1800$ abgedeckt. Die instationären Kräfte auf eine Kugel werden im gesamten untersuchten Reynoldszahlbereich von der potentialtheoretischen zusätzliche-Masse-Kraft (engl: added-mass force) dominiert. Reibungskräfte und die Basset-Kraft (engl. history-force) spielen keine Rolle. Lediglich der von Odar und Hamilton eingeführte Gewichtungsfaktor $C_{A}^{*}$ der zusätzliche-Masse-Kraft beeinflusst die Kraft auf die Kugel und hängt nur von der Beschleunigungszahl ab, welche als Verhältnis von Kugelgröße zur ausgelenkten Luft ausgedrückt werden kann.

Für potentialtheoretische Strömungen gilt $C_{A}^{*}=2$. Bereits für sehr kleine Beschleunigungszahlen nähert sich $C_{A}^{*}$ einem Grenzwert von $C_{A}^{*}=2,3$ an. Da $C_{A}^{*}$ von der Reynoldszahl unabhängig ist, kann sowohl im skalierten Modellexperiment als auch im Originalmaßstab $C_{A}^{*}=2,3$ gesetzt werden.

Ein Vergleich der Messergebnisse aus den TSG-Messungen mit den theoretischen Kräften sowohl mit dem potentialtheoretischen Wert $C_{A}^{*}=2$ als auch mit dem empirisch bestimmten Wert $C_{A}^{*}=2,3$ zeigt, dass die Abweichung zwischen gemessener und theoretischer Kraftamplitude signifikant reduziert werden kann, die wirkenden Kräfte also sehr viel genauer vorhergesagt werden können. 



\title{
Aerodynamic loads on resting objects induced by fast-moving near-ground bodies
}

\begin{abstract}
In an unsteady flow field pressure variations lead to aerodynamic loads on objects like spheres or disks. Under the assumption of inviscid flow, the potential flow theory is a suitable method to predict aerodynamic loads on those objects. In an unsteady flow field of a passing train aerodynamic loads are able to destabilize waiting passengers on a platform or trackside workers on open track. In literature neither real train geometries nor the object geometry were considered so far.

In this thesis a more flexible and more precise analytical model is derived. This model is able to predict the aerodynamic loads on an object in unsteady flow induced by the pressure pulse of a passing train head for model-scale experiments as well as for full-scale measurements adequately.

In order to validate the accuracy of the analytical model model-scale experiments are performed in the Tunnel-Simulation Facility Göttingen.

Additional experiments in the unsteady flow field of a huge loudspeaker are performed in order to investigate the physical effects causing the loads on spheres. The validity of the equation by Basset, Boussinesq, and Oseen with the modifications by Odar and Hamilton is examined. Only small Reynolds numbers up to $R e=100$ were investigated so far in literature. In this thesis a Reynolds number range from $R e=200$ until $R e=1800$ is considered for the first time.

The so called added-mass force dominates the force on the spheres in all measured cases. Neither drag forces nor the history force plays a major role. Only the factor $C_{A}^{*}$ of the added-mass force influences the loads on the sphere and depends on the so-called acceleration number which can be expressed as the ratio between the displacement of the fluid and the diameter of the sphere.

For a potential flow it is $C_{A}^{*}=2$. For small acceleration numbers $C_{A}^{*}$ approaches a threshold value which is $C_{A}^{*}=2,3$. Since $C_{A}^{*}$ is independent of the Reynolds number it can be set $C_{A}^{*}=2,3$ for model-scale experiments as well as for full-scale measurements. A comparison with the results obtained from the TSG measurements shows that the deviations between experiment and theory can be reduced significantly by setting $C_{A}^{*}=$ 2,3 instead of $C_{A}^{*}=2$. Therefore, aerodynamic loads on spheres induced by the pressure pulse of a passing train can be predicted more precisely.
\end{abstract}



Tabellenverzeichnis $\quad$ XI

$\begin{array}{lll}\text { Nomenklatur } & \text { XIII }\end{array}$

$\begin{array}{ll}\text { 1. Einleitung } & 1\end{array}$

2. Analytische Berechnung der Zugvorbeifahrt mittels Potentialtheorie 5

2.1. Grundgleichungen der reibungsfreien Strömung . . . . . . . . . . . . 6

2.2. Superposition elementarer Potentiallösungen: Stromlinienkörper . . . . . . 7

2.2.1. Schlanke Zuggeometrie . . . . . . . . . . . . . . . . 7

2.2.2. Stumpfe Zuggeometrie . . . . . . . . . . . . . . . . . 13

2.2.3. Kugel . . . . . . . . . . . . . . . . . . . . 15

2.3. Simulation der Vorbeifahrt und daraus resultierende Kraft auf Objekte . . 16

2.3.1. Kraft auf eine Kugel . . . . . . . . . . . . . . . . . . 16

2.3.2. Berechnung der Kraft auf eine Kugel mit akustischem Ansatz . . . 19

2.3.3. Kräfte auf ebene Platten . . . . . . . . . . . . . . . . . 21

2.4. Vergleich der aerodynamischen Lasten durch verschiedene Zuggeometrien 23

3. Die Tunnel-Simulations-Anlage Göttingen (TSG) 27

3.1. Aufbau der Versuchsanlage . . . . . . . . . . . . . . . . . . 27

3.2. Verwendete Modellzüge . . . . . . . . . . . . . . . . . . . . . 32

4. Geschwindigkeitsmessungen an der TSG mithilfe von Hitzdrahtanemometrie $\mathbf{3 3}$

4.1. Verwendete Messtechnik . . . . . . . . . . . . . . . . . 33

4.2. Kalibrierung . . . . . . . . . . . . . . . . . . 35

4.3. Winkelkalibrierung . . . . . . . . . . . . . . . 35

4.4. Aufbau und Durchführung der Vorbeifahrtmessungen . . . . . . . . . . . 38 
Inhaltsverzeichnis

4.5. Auswertung und Diskussion . . . . . . . . . . . . . . . . . . . 39

5. Instationäre Kraftmessungen - Verwendete Messtechniken 41

5.1. Eindimensionales Kraftmesselement . . . . . . . . . . . . . . . . . . . 41

5.1.1. Kalibrierung des Kraftmesselements . . . . . . . . . . . . . . 41

5.1.2. Filterung des Spannungssignals . . . . . . . . . . . . . . . . . . . 44

5.2. Optische Objektverfolgung . . . . . . . . . . . . . . . . . . . 49

6. Instationäre Kraftmessungen an der TSG $\mathbf{5 3}$

6.1. Kraftmessungen mit einem Kraftmesselement: ICE3 mit Bahndamm . . . 53

6.1.1. Aufbau und Durchführung . . . . . . . . . . . . . . . 53

6.1.2. Ergebnisse . . . . . . . . . . . . . . . . 54

6.2. Exkurs: Iterative Anpassung der theoretischen Zugkontur . . . . . . . . . 59

6.3. Kraftmessungen mit einem Kraftmesselement: Potentialzug mit flachem

Boden . . . . . . . . . . . . . . . . . . 62

6.3.1. Aufbau und Durchführung. . . . . . . . . . . . . . . 62

6.3.2. Ergebnisse . . . . . . . . . . . . . . . . 65

6.4. Instationäre Kraftmessungen mit optischer Objektverfolgung . . . . . . 74

6.4.1. Versuchsaufbau und Durchführung . . . . . . . . . . . . 75

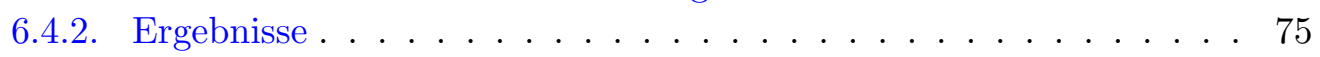

6.5. Diskussion . . . . . . . . . . . . . . . . . . 79

7. Kräfte auf Objekte in periodischen instationären Strömungen 83

7.1. Theoretische Überlegungen . . . . . . . . . . . . . . . . . . . . 83

7.2. Experimentelle Untersuchungen . . . . . . . . . . . . . . . . . 85

7.2.1. Versuchsanordnung . . . . . . . . . . . . . . 86 8

7.3. Vermessung des Geschwindigkeitsfeldes des Lautsprechers . . . . . . . . 87

7.4. Messung der instationären Kraft auf eine Kugel mithilfe eines Kraftmess-

elements . . . . . . . . . . . . . . . . . . . . 91

7.4.1. Resonanzen der Kraftwaage . . . . . . . . . . . . . . . . . 91

7.4.2. Einfluss der Kraftwaage auf die gemessene Kraft . . . . . . . . . 93

7.4.3. Durchführung der Kraftmessung . . . . . . . . . . . . . . . 95

7.4.4. Auswertung und Bestimmung der Kraftanteile . . . . . . . . . . . 95

7.4.5. Ergebnisse . . . . . . . . . . . . . . . . . . . . 97

7.5. Messung des Druckgradienten an der Kugelposition . . . . . . . . . . . . 103

7.6. Messung der Kraft auf eine Kugel mithilfe von optischer Objektverfolgung 103

7.6.1. Aufbau und Durchführung . . . . . . . . . . . . . . . . 103

7.6.2. Auswertung . . . . . . . . . . . . . . . . . . 104

7.6.3. Ergebnisse . . . . . . . . . . . . . . . . 106

7.7. Diskussion . . . . . . . . . . . . . . . . . . 108

$\begin{array}{ll}\text { 8. Zusammenfassung und Ausblick } & 111\end{array}$

$\begin{array}{lr}\text { Literaturverzeichnis } & 115\end{array}$

II 
Inhaltsverzeichnis

$\begin{array}{ll}\text { Appendices } & 121\end{array}$

$\begin{array}{ll}\text { A. Geschwindigkeitskurven der Vorbeifahrtmessungen } & 123\end{array}$

$\begin{array}{ll}\text { B. Kraftkurven der Vorbeifahrtmessungen } & 127\end{array}$

$\begin{array}{ll}\text { C. Lautsprechermessungen } & 131\end{array}$ 

2.1. Beispiel für eine Kontur mit zu weit auseinander liegenden Quellen (schematische Darstellung). . . . . . . . . . . . . . . . . . 99

2.2. Vergleich der Konturen des Potentialszugs (schwarz) mit Quellpositionen und Trägerbalken (grau) und des ICE3 (rot). . . . . . . . . . . . . 9

2.3. Potentialkörper mit Spiegelzug, Quellen und Senken und den daraus resultierenden Stromlinien, Schnitt durch die x-z-Ebene. . . . . . . . . . . . 11

2.4. 3D-Kontur des Potentialzugs. . . . . . . . . . . . . . . . 12

2.5. Dreidimensionale Darstellung des Potentialzugkopfs. Die farbige Skala gibt den $c_{p}$-Wert auf der Oberfläche an. . . . . . . . . . . . . . 12

2.6. Darstellung des entstehenden Strömungsfeldes durch eine rechteckige Quellenverteilung (aus $[1]$, bearbeitet). . . . . . . . . . . . . 13

2.7. Berechnete Kontur für einen Kastenzug mit $a=4 \mathrm{~cm}, b=5 \mathrm{~cm}$ und $A=0,016 \mathrm{~m}^{2} \ldots \ldots \ldots \ldots \ldots \ldots \ldots \ldots \ldots$

2.8. Blick von oben auf den Potentialzug und die Kugel $R=2 \mathrm{~cm}$ bei $y_{M}=$ $10 \mathrm{~cm}$ an der Position $x=0 . \ldots \ldots \ldots \ldots \ldots$

2.9. Getrennte Berechnung des Kraftbeiwertes aus dem stationären und dem instationärem Anteil der Bernoulli-Gleichung (schwarz: Gesamtkraft, rot: instationärer Anteil, blau: stationärer Anteil). . . . . . . . . . . . . . 18

2.10. Kugel im Druckfeld eines fahrenden Zuges (schematische Darstellung). . . 19

2.11. Kraft auf die Kugel durch die Kopfwelle, Vergleich der beiden theoretischen Modelle. . . . . . . . . . . . . . . . . . . . . 22

2.12. Betrag der Geschwindigkeiten am Ort der Kugel, induziert durch drei verschiedene Zugkkopfgeometrien: Punktquelle, Dreifachquelle, Kasten$\operatorname{zug}\left(y_{M}=0,12 \mathrm{~m}\right.$ und $\left.u_{\infty}=40 \mathrm{~m} / \mathrm{s}\right) \ldots \ldots \ldots \ldots 24$

2.13. Vergleich der Kräfte auf eine Kugel $R=2 \mathrm{~cm}$, bei $y_{M}=12 \mathrm{~cm}$ und $u_{\infty}=40 \mathrm{~m} / \mathrm{s}$ induziert durch verschiedene potentialtheoretischer Zuggeometrien: Punktquelle, Mehrfachquelle, Kastenzug. . . . . . . . . . . . . . 25 


\section{ABBILDUNGSVERZEICHNIS}

3.1. Schematische Darstellung der TSG [2] . . . . . . . . . . . . . . . . 28

3.2. Katapult zur Beschleunigung der Modelle in der Versuchsanlage. . . . . . 28

3.3. Schematische Darstellung der Kraftübertragung auf das Modell durch einen Hilfsschlitten [2] . . . . . . . . . . . . . . . . . . . . . . . . 29

3.4. Messstrecke mit Seitenwindmodul. . . . . . . . . . . . . . . 30

3.5. Ansicht der Bremsbox der TSG. . . . . . . . . . . . . . . 31

3.6. Für die Vorbeifahrtmessungen verwendete Zugmodelle: a) Potentialzug, b) ICE3 und c) Next Generation Train (NGT) . . . . . . . . . . . . . . . . 32

4.1. Schematische Darstellung der verwendeten Ein-Drahtsonden. . . . . . . . 34

4.2. Blockschaltbild CTA, der Widerstand des Drahtes $R_{w}$ wird auf $R_{0}$ abgeglichen. . . . . . . . . . . . . . . . . . 35

4.3. Geschwindigkeitskalibrierung der Hitzdrahtsonde mithilfe des King'schen Gesetzes. . . . . . . . . . . . . . . . . 36

4.4. Theoretischer Winkel der Strömung bei der Vorbeifahrt (Gl. 4.3). Die Stelle $x=0$ bezeichnet den Ort, an der sich die Zugnase auf Höhe der Hitzdrahtsonde befindet. . . . . . . . . . . . . . . . 37

4.5. Experimenteller Aufbau der Winkelkalibrierung der Hitzdraht Eindrahtsonde im Seitenwindmodul in der TSG. . . . . . . . . . . . . . . 37

4.6. Korrekturkurve für den Hitzdrähte in Fahrtrichtung (Hitzdraht 2 in Abb. 4.7) (Gl. 4.4). Für den Hitzdraht lateral zur Fahrtrichtung (Hitzdraht 1 in Abb. 4.7) wird die Kurve entsprechend um $90^{\circ}$ verschoben. . . . . . . . 38

4.7. Schematische Darstellung des Versuchsaufbau in der Messstrecke der TSG zur Geschwindigkeitsmessung mittels Hitzdrahtanemometrie. . . . . . . . 38

4.8. Vergleich unkorrigiertes und korrigiertes Geschwindigkeitssignal für $y_{M}=$ $10 \mathrm{~cm}, z_{M}=4,5 \mathrm{~cm}, u_{\infty}=40,48 \mathrm{~m} / \mathrm{s} \ldots \ldots \ldots . \ldots . \ldots 39$

5.1. Schematische Darstellung des Kraftmesselements. . . . . . . . . . . . . . . 42

5.2. Graphische Darstellung der Filterfunktion $\psi$. . . . . . . . . . . . . 45

5.3. Darstellung des Ergenbnisses der Signalverarbeitung. Das gemessene Signal $U(t)$ ist rot dargestellt, das gefilterte Signal in schwarz und das Ergebnis der Entfaltung $U(\omega)$ in blau. . . . . . . . . . . . . . 47

5.4. Exemplarische Auswertung für einen Vorbeifahrtversuch. . . . . . . . . . . 48

5.5. Demonstration der Kantendetektion anhand eines Beispielbildes. . . . . . 50

5.6. Schematische Darstellung der Verfolgung der Kugel während der Vorbeifahrt des Zuges. Die gepunktete Linie stellt die gefundenen Kantenpixel dar und die durchgezogene Linie die analytische Modellbeschreibung des Kreises. . . . . . . . . . . . . . . . . . . . . . 50

6.1. Versuchsaufbau für die Kraftmessungen mit dem 1D-Kraftmesselement und dem ICE3-Modell an der TSG. . . . . . . . . . . . . . . . . . . . . 54

6.2. Vergleich der gemessenen Kraft auf den Tischtennisball mit der potentialtheoretisch berechneten Kraft. . . . . . . . . . . . . . . . . 55

6.3. Graphische Darstellung der Ermittelung von $\Delta F \ldots \ldots \ldots$. . . . . . 56 
6.4. Kräfte auf einen Tischtennisball $(R=2 \mathrm{~cm})$ bei verschiedenen Gleisabständen und einer Fahrtgeschwindigkeit von $u_{\infty}=37,9 \mathrm{~m} / \mathrm{s} . \ldots . . .56$

6.5. Kräfte auf Styroporkugeln unterschiedlicher Radien bei einem Gleisabstand von $y_{M}=11 \mathrm{~cm}$ und einer Fahrtgeschwindigkeitkeit von $u_{\infty}=$ $37,8 \mathrm{~m} / \mathrm{s} . \ldots \ldots \ldots \ldots \ldots \ldots \ldots \ldots$

6.6. Vergleich der Kräfte auf die Kugel für die angepasste und nicht-angepasste Zuggeometrie sowie der experimentellen Kraftkurve. . . . . . . . . . . . 60

6.7. Vergleich der Kräfte auf die Kugel für die angepasste und nicht-angepasste Zuggeometrie sowie der experimentellen Kraftkurve als Polardiagramm. . 61

6.8. Vergleich der angepassten Zugkonturen (drei bzw. fünf Quellen, blaue bzw. magentafarbene Linie) mit der nicht angepassten Zugkontur mit drei Quellen (schwarz) und der Kontur des ICE3-Modells (rot). . . . . . . . . . 62

6.9. Versuchsaufbau zur instationären Kraftmessung in der TSG. . . . . . . . . 63

6.10. Gemittelte Kraft mit RMS-Wert über fünf Fahrten bei der Vorbeifahrt des Potentialzugs auf die Styroporkugel $(R=2 \mathrm{~cm})$, bei einem Gleisabstand von $y_{M}=12 \mathrm{~cm}$ und einer Fahrtgeschwindigkeit von $u_{\infty}=40 \mathrm{~m} / \mathrm{s}$ verglichen mit der Potentialtheorie. . . . . . . . . . . . . . . .

6.11. Gemittelte Kraft über fünf Fahrten mit RMS-Wert (schwarze Linie) bei der Vorbeifahrt des Potentialzugs auf die Karbonscheibe $(R=2 \mathrm{~cm})$, bei einem Gleisabstand von $y_{M}=12 \mathrm{~cm}$ und einer Fahrtgeschwindigkeit von $u_{\infty}=40 \mathrm{~m} / \mathrm{s}$ verglichen mit der Potentialtheorie für Kreisscheiben (blaue Linie) und rechteckige Platten (rote Linie). . . . . . . . . . . . . . .

6.12. Kraft quer zur Fahrtrichtung auf die Scheibe, verglichen mit stationärer und instationärer Theorie. . . . . . . . . . . . . . . . . .

6.13. Kräfte auf Styroporkugeln und Karbonscheiben unterschiedlicher Radien bei einem Gleisabstand von $y_{M}=12 \mathrm{~cm}$ und einer Fahrtgeschwindigkeit-

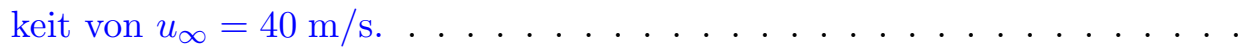

6.14. Kräfte auf eine Kugel bzw. Scheibe $(R=2 \mathrm{~cm})$ bei verschiedenen Gleisabständen und einer Fahrtgeschwindigkeit von $u_{\infty}=40 \mathrm{~m} / \mathrm{s}$. . . . . . . 70

6.15. Kraftamplitude bei der Kugel für verschiedene Zuggeschwindigkeiten $u_{\infty}$. $\quad 71$

6.16. Vergleich der experimentellen Kräfte auf die Kugel für verschiedene Höhen des Kugelmittelpunktes $(R=2 \mathrm{~cm})$ über der Schienenoberkante mit der potentialtheoretischen Kraftkurve für $z_{M}=6 \mathrm{~cm} . \ldots \ldots \ldots$. . . . 72

6.17. Abhängigkeit der Phasenlänge $\Delta x$ von der Höhe der Kugel über der Schienenoberkante. . . . . . . . . . . . . . . . . . 73

6.18. Vergleich der induzierten Kraft auf eine $\operatorname{Kugel}(R=2 \mathrm{~cm})$ durch verschiedene Zugköpfe bei einem Gleisabstand von $y_{M}=12 \mathrm{~cm}, z_{M}=4,5 \mathrm{~cm}$ und einer Fahrtgeschwindigkeit von $u_{\infty}=40 \mathrm{~m} / \mathrm{s} \ldots \ldots \ldots . \ldots 74$

6.19. Schematischer Versuchsaufbau zur instationären Kraftmessung mithilfe von optischer Objektverfolgung in der TSG. . . . . . . . . . . . . . 75

6.20. Aufnahme der Vorbeifahrt des Potentialzuges an der Kugel $R=25 \mathrm{~mm}$ zum Zeitpunkt $t=0,4 \mathrm{~ms}$. Die gefundenen Kantenpixel sind eingezeichnet. 76 


\section{ABBILDUNGSVERZEICHNIS}

6.21. Vergleich der Kraftbeiwerte auf eine Kugel $R=25 \mathrm{~mm}$, bei $y_{M}=12 \mathrm{~cm}$ und $u_{\infty}=40 \mathrm{~m} / \mathrm{s}$ für verschiedene Messtechniken: Kraftwaage (blau) und optische Objektverfolgung (rot) im Vergleich mit der Potentialtheorie (schwarz). . . . . . . . . . . . . . . . . . 78

6.22. Vergleich der Kraftänderung $\mathrm{d} c_{F} / \mathrm{d} x$ auf eine Kugel $R=25 \mathrm{~mm}$, bei $y_{M}=12 \mathrm{~cm}$ und $u_{\infty}=40 \mathrm{~m} / \mathrm{s}$ für verschiedene Messtechniken: Kraftwaage (blau) und optische Objektverfolgung (rot) im Vergleich mit der Potentialtheorie $($ schwarz $) \ldots \ldots \ldots . \ldots . \ldots . \ldots 78$

7.1. Versuchsaufbau der Geschwindigkeitsmessungen mit Microflown-Sonden. . 88

7.2. Geschwindigkeitsfeld bei $f=30 \mathrm{~Hz}$. . . . . . . . . . . . . . . 89

7.3. Geschwindigkeits-Frequenz-Spektrum bei verschiedenen Höhen über der Membran, relativ zur Kugelposition, gemessen mit Miroflown-Sonden. $0 \mathrm{~cm}$ entspricht der Höhe der Kugel oberhalb der Membran. . . . . . . . . 90

7.4. Vergleich der Geschwindigkeiten an der Membran (Laservibrometer) und auf Kugelhöhe (Microflown-Sonden). . . . . . . . . . . . . . . . . 90

7.5. Versuchsaufbau der Kraftmessungen im instationären Lautsprecherfeld mithilfe eines Kraftmesselements. . . . . . . . . . . . . . . . . . . . . . 91

7.6. Resonanzen des Masse-Feder-Systems: Waage-Kugel mit unterschiedlichen Halterungen und Waagensteifigkeiten. . . . . . . . . . . . . . . 92

7.7. Vergleich der Kraft auf die Waage mit $R=20 \mathrm{~mm}$ Kugel (blaue Kurve) und ohne angeschraubte Kugel (schwarze Kurve) bei $\mathrm{f}=71 \mathrm{~Hz}$. . . . . . . . 94

7.8. Gemessene Geschwindigkeit (bereits normiert, violette Kurve) und gemessene Kraft (grüne Kurve) bei $f=71 \mathrm{~Hz}$ und der $R=20 \mathrm{~mm}$ Kugel. . . . 96

7.9. Kraftanteile im Vergleich mit der gemessenen Kraft für $f=110 \mathrm{~Hz}$, $R=20 \mathrm{~mm}$ und einer Amplitude von $200 \mathrm{mV}$. Die Position $t_{\text {mess }}$ zur Bestimmung von $C_{A}^{*}$ ist ebenfalls eingezeichnet. . . . . . . . . . . 98

7.10. Experimentell ermittelter Wert für $C_{A}^{*}$ für verschiedene Frequenzen. .. . 99

7.11. Vergleich der Anpassungsfunktion nach Odar und Hamilton $(\mathrm{OH})$ mit den experimentellen Werten für $C_{A}^{*}$ und den experimentellen Werten von Odar und Hamilton. . . . . . . . . . . . . . . . . . . . . . . . . 100

7.12. Experimentell ermittelter Wert für $C_{H}$ für verschiedene Frequenzen. . . . 101

7.13. Versuchsaufbau für die Messung des Druckgradienten mithife von zwei Drucksensoren im Abstand von $2 \mathrm{~cm}$. . . . . . . . . . . . . . . . 101

7.14. Gemessene Drucksignale und daraus berechneter Druckgradient bei $f=$ $30 \mathrm{~Hz}$ und einer Amplitude von $500 \mathrm{mV}$. . . . . . . . . . . . . . 102

7.15. Vergleich der Frequenz-Geschwindigkeits-Spektren der Drucksensoren und des Laservibrometers bei einer Amplitude von 500 mV an der Kugelposition.102

7.16. Versuchsaufbau für die Kraftmessung im Lautsprecherfeld mithilfe von optischer Objektverfolgung. . . . . . . . . . . . . . . . . . . . 104

7.17. Verfolgte Bewegung der $R=15 \mathrm{~mm}$-Kugel bei $f=30 \mathrm{~Hz}$ und einer Amplitude von $1000 \mathrm{mV}$. . . . . . . . . . . . . . . 105 
7.18. Berechnete Kraft aus der gemessenen Kugeltrajektorie mittels optischer Objektverfolgung bei $f=30 \mathrm{~Hz}, A m p=1000 \mathrm{mV}, R=15 \mathrm{~mm}$. . . . . 107

7.19. Vergleich der Messwerte und angepassten Funktionsgleichungen für $C_{A}^{*}$ in Abhängigkeit von $A c$, ermittelt mit der Kraftwaage sowie der optischen Objektverfolgung mit der empirischen Funktion von Odar und Hamilton

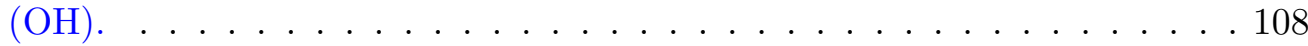

7.20. Vergleich der gemessenen Kraft auf eine Kugel $(R=20 \mathrm{~mm})$ bei der Vorbeifahrt $\left(u_{\infty}=40 \mathrm{~m} / \mathrm{s}, y_{M}=12 \mathrm{~cm}\right)$ im Vergleich mit der potentialtheoretischen Kraftvorhersage mit $C_{A}^{*}=2$ und $C_{A}^{*}=2,3 . \ldots \ldots 110$

A.1. Vergleich unkorrigiertes und korrigiertes Geschwindigkeitssignal für $y_{M}=$

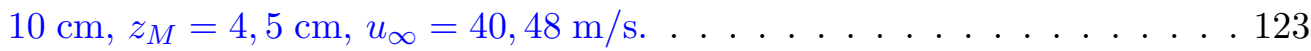

A.2. Vergleich unkorrigiertes und korrigiertes Geschwindigkeitssignal für $y_{M}=$

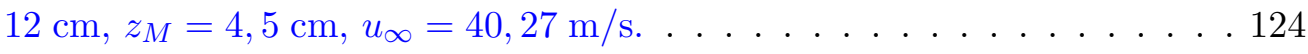

A.3. Vergleich unkorrigiertes und korrigiertes Geschwindigkeitssignal für $y_{M}=$

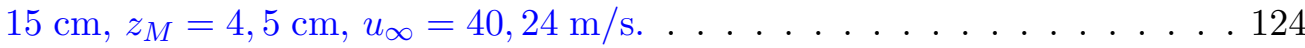

A.4. Vergleich unkorrigiertes und korrigiertes Geschwindigkeitssignal für $y_{M}=$

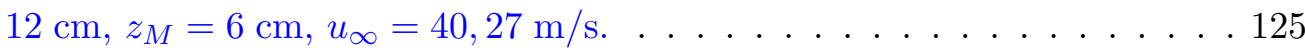

A.5. Vergleich unkorrigiertes und korrigiertes Geschwindigkeitssignal für $y_{M}=$

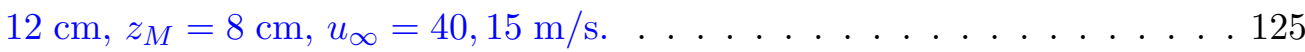

A.6. Vergleich unkorrigiertes und korrigiertes Geschwindigkeitssignal für $y_{M}=$ $12 \mathrm{~cm}, z_{M}=10 \mathrm{~cm}, u_{\infty}=40,33 \mathrm{~m} / \mathrm{s}$. Hitzdraht 2 ist bei diesem Versuch leider kaputt gegangen. . . . . . . . . . . . . . . . . . 126

B.1. Vergleich der experimentellen Kräfte induziert vom ICE3-Modell auf die Kugel $(R=2 \mathrm{~cm})$ für verschiedene Gleisabstände mit den jeweiligen potentialtheoretischen Kraftkurven für $z_{M}=4,5 \mathrm{~cm}$ und $u_{\infty}=37,7 \mathrm{~m} / \mathrm{s} . .127$

B.2. Vergleich der experimentellen Kräfte induziert vom ICE3-Modell auf verschiedene Kugeln mit den jeweiligen potentialtheoretischen Kraftkurven für $z_{M}=4,5 \mathrm{~cm}, u_{\infty}=37,7 \mathrm{~m} / \mathrm{s}$ und $y_{M}=11 \mathrm{~cm} \ldots \ldots 128$

B.3. Vergleich der experimentellen Kräfte auf die Kugel $(R=2 \mathrm{~cm})$ induziert vom Potentialmodell für verschiedene Fahrtgeschwindigkeiten mit den jeweiligen potentialtheoretischen Kraftkurven für $z_{M}=4,5 \mathrm{~cm}$ und

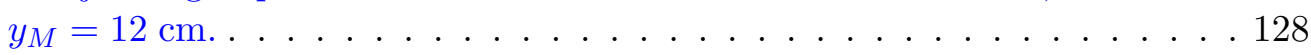

B.4. Vergleich der experimentellen Kräfte auf die Kugel $(R=2 \mathrm{~cm})$ induziert vom Potentialmodell für verschiedene Gleisabstände mit den jeweiligen potentialtheoretischen Kraftkurven für $z_{M}=4,5 \mathrm{~cm}$ und $u_{\infty}=40 \mathrm{~m} / \mathrm{s}$.

B.5. Vergleich der experimentellen Kräfte induziert vom Potentialmodell auf verschiedene Kugeln mit den jeweiligen potentialtheoretischen Kraftkurven für $z_{M}=4,5 \mathrm{~cm}, u_{\infty}=40 \mathrm{~m} / \mathrm{s}$ und $y_{M}=12 \mathrm{~cm} . \ldots \ldots . . \ldots 129$

B.6. Vergleich der experimentellen Kräfte induziert vom Potentialmodell auf verschiedene Scheiben mit den jeweiligen potentialtheoretischen Kraftkurven für $z_{M}=4,5 \mathrm{~cm}, u_{\infty}=40 \mathrm{~m} / \mathrm{s}$ und $y_{M}=12 \mathrm{~cm} . \ldots \ldots 130$ 


\section{ABBILDUNGSVERZEICHNIS}

B.7. Vergleich der experimentellen Kräfte induziert vom Potentialmodell auf die Scheibe $(R=2 \mathrm{~cm})$ für verschiedene Gleisabstände mit den jeweiligen potentialtheoretischen Kraftkurven für $z_{M}=4,5 \mathrm{~cm}$ und $u_{\infty}=40 \mathrm{~m} / \mathrm{s} . \quad .130$

C.1. Kraftanteile im Vergleich mit der gemessenen Kraft für $f=30 \mathrm{~Hz}$. . . . . 133

C.2. Kraftanteile im Vergleich mit der gemessenen Kraft für $f=71 \mathrm{~Hz}$. . . . . 134

C.3. Kraftanteile im Vergleich mit der gemessenen Kraft für $f=110 \mathrm{~Hz}$. . . . 135

C.4. Kraftanteile im Vergleich mit der gemessenen Kraft für $f=110 \mathrm{~Hz}$. . . . 136 
2.1. Vergleich der Geometrien des ICE3-Modells und des berechneten Potentialzugs im Maßstab 1:25. . . . . . . . . . . . . . . . . . 8

2.2. Positionen der Quellen und Senken und ihre Quellstärken. . . . . . . . . . 9

3.1. Vergleich der Geometrien der verwendeten Modellzüge. . . . . . . . . . . . 32

4.1. Messmatrix Hitzdrahtmessungen, $u_{\infty} \approx 40 \mathrm{~m} / \mathrm{s} . y_{M}$ ist die Entfernung der Ballmitte von der Gleismitte und $z_{M}$ die Position der Ballmitte über der Schienenoberkante. . . . . . . . . . . . . . . . . . 39

6.1. Variationskoeffizient $\vartheta$ zwischen den theoretischen und dem experimentellen Kraftverläufen in x- und in y-Richtung für die verschiedenen Zuggeometrien (angepasst und nicht angepasst) . . . . . . . . . . . . . 62

6.2. Messmatrix Potentialzug, Kugel. . . . . . . . . . . . . . . . . . . 64

6.3. Messmatrix Potentialzug, Scheibe, $u_{\infty}=40 \mathrm{~m} / \mathrm{s} . \ldots \ldots \ldots$

7.1. Resonanzfrequenzen von Waage, Halterung und Kugel für verschiedene Kugelradien und Halterungen/Waage-Kombinationen. . . . . . . . . . . . 92

7.2. Vergleich des Anteils der Kraft auf die Waage ohne Kugel im Vergleich zur jeweiligen Kugelgröße. . . . . . . . . . . . . . . . . . . . . . 94

7.3. Massen der verwendeten Kugeln. . . . . . . . . . . . . . . . . . . . 105

C.1. Normierfaktoren für die Geschwindigkeit auf Kugelhöhe. . . . . . . . . . . 131

C.2. Ergebnisse: Messung mit der Kraftwaage. . . . . . . . . . . . . . . . . 133

C.3. Ergebnisse der Kraftmessung im instationären Lautsprecherfeld mit optischer Objektverfolgung. . . . . . . . . . . . . . . . . 137 



\section{Lateinische Buchstaben}

\begin{tabular}{lll} 
Variable & Bedeutung & Einheit \\
\hline $\mathrm{a}$ & Beschleunigung & $\mathrm{m} / \mathrm{s}^{2}$ \\
$\mathrm{~A}$ & Querschnittsfläche & $\mathrm{m}^{2}$ \\
$\mathrm{~b}$ & Abstand zur Spiegelquelle & $\mathrm{m}$ \\
$\mathrm{c}$ & Schallgeschwindigkeit & $\mathrm{m} / \mathrm{s}$ \\
$c_{1}$ & Konstante & {$[\mathrm{a} . \mathrm{u}]$.} \\
$\mathrm{d}$ & Dicke der Scheibe & $\mathrm{m}$ \\
$\mathrm{D}$ & Zugbreite & $\mathrm{m}$ \\
$E_{k i n}$ & kinetische Energie & $\mathrm{Nm}$ \\
$\mathrm{F}$ & Kraft & $\mathrm{N}$ \\
$F_{A}$ & zusätzliche-Masse-Kraft & $\mathrm{N}$ \\
$F_{D}$ & Reibungskraft & $\mathrm{N}$ \\
$F_{H}$ & Basset-Kraft & $\mathrm{N}$ \\
$\mathrm{f}$ & Frequenz & $\mathrm{s}^{-1}$ \\
$\mathrm{~h}$ & Höhe & $\mathrm{m}$ \\
$\mathrm{I}$ & Strom & $\mathrm{A}$ \\
$\mathrm{k}$ & Wellenzahl & - \\
$\vec{n}$ & Normalenvektor & - \\
$\mathrm{m}$ & Masse & $\mathrm{kg}$ \\
$\mathrm{p}$ & Druck & $\mathrm{N} / \mathrm{m}^{2}$ \\
$\mathrm{Q}$ & Quellstärke & $\mathrm{m} / \mathrm{s}$ \\
$\mathrm{R}$ & Kugelradius & $\mathrm{m}$ \\
$R_{w}$ & Ohm'scher Widerstand & $\Omega$ \\
$\mathrm{t}$ & Zeit & $\mathrm{s}$ \\
$\mathrm{T}$ & Temperatur & $\mathrm{K}$ \\
$\mathrm{U}$ & Spannung & $\mathrm{V}$ \\
$\vec{u}$ & Geschwindigkeitsvektor & $\mathrm{m} / \mathrm{s}$ \\
$u_{\infty}$ & Anströmgeschwindigkeit & $\mathrm{m} / \mathrm{s}$ \\
$\mathrm{u}$ & Geschwindigkeit in $x$-Richtung & $\mathrm{m} / \mathrm{s}$ \\
& &
\end{tabular}




$\begin{array}{lll}\mathrm{v} & \text { Geschwindigkeit in } y \text {-Richtung } & \mathrm{m} / \mathrm{s} \\ \mathrm{w} & \text { Geschwindigkeit in z-Richtung } & \mathrm{m} / \mathrm{s} \\ \mathrm{x} & \text { Fahrtrichtung } & \mathrm{m} \\ \vec{x} & \text { Ortsvektor } & \mathrm{m} \\ \vec{x}_{M} & \text { Mittelpunkt der Kugel } & \mathrm{m} \\ \mathrm{y} & \text { Lateral zur Fahrtrichtung } & \mathrm{m} \\ y_{M} & \text { Gleisabstand vom Kugelmittelpunkt } & \mathrm{m} \\ & \text { zur Gleismitte } & \\ \mathrm{z} & \text { Vertikal zur Fahrtrichtung } & \mathrm{m}\end{array}$

\section{Griechische Buchstaben}

\begin{tabular}{lll} 
Variable & Bedeutung & Einheit \\
\hline$\beta$ & Abklingkonstante & $\mathrm{s}^{-1}$ \\
$\rho$ & Dichte & $\mathrm{kg} / \mathrm{m}^{3}$ \\
$\varphi$ & Winkel & $\mathrm{rad}$ \\
$\phi$ & Geschwindigkeitspotential & $\mathrm{m}^{2} / \mathrm{s}$ \\
$\Phi$ & Anströmwinkel & $\circ$ \\
$\lambda$ & Wellenlänge & $\mathrm{m}$ \\
$\mu$ & dynamische Viskosität & $\mathrm{N} / \mathrm{m}^{2} \cdot \mathrm{s}$ \\
$\vartheta$ & Variationskoeffizient & - \\
$\omega$ & Kreisfrequenz & $\mathrm{s}^{-1}$
\end{tabular}

\section{Abkürzungen}

\begin{tabular}{ll} 
Abkürzung & Bedeutung \\
\hline 1D & Eindimensional \\
2D & Zweidimensional \\
3D & Dreidimensional \\
BBO & Basset, Boussinesq und Oseen \\
CFD & Computional Fluid Dynamics \\
DLR & Deutsches Zentrum für Luft- und Raumfahrt \\
ICE & Inter City Express \\
OH & Odar und Hamilton \\
TSG & Tunnel Simulationsanlage Göttingen
\end{tabular}




\section{Indices}

\begin{tabular}{ll} 
Index & Bedeutung \\
\hline 0 & Umgebungsbedingung \\
Aufn & Aufnahme \\
D & Dipol \\
F & Kraft \\
eff & effektiv \\
ein & einfallende Welle \\
ges & gestreute Welle \\
K & Kugel \\
M & Monopol \\
max & Maximum \\
min & Minimum \\
tot & total \\
$\infty$ & Umgebungsbedingung
\end{tabular}

\section{Dimensionslose Größen}

\begin{tabular}{lll} 
Abkürzung & Name & Formel \\
\hline$A c$ & Beschleunigungszahl & $\frac{u^{2}}{a D}$ \\
$c_{F}$ & Kraftbeiwert & $F /\left(\frac{1}{2} \rho_{0} u_{\infty}^{2} A\right)$ \\
$c_{p}$ & Druckbeiwert & $p /\left(\frac{1}{2} \rho_{0} u_{\infty}^{2}\right)$ \\
$M$ & Mach Zahl & $u / c$ \\
$R e$ & Reynolds Zahl & $(\rho \cdot u \cdot d) / \mu$
\end{tabular}



KAPITEL 1

Einleitung

In einem instationären Strömungsfeld üben Druckschwankungen aerodynamische Kräfte auf Körper, wie Kugeln oder Scheiben aus. In reibungsfreien Strömungen können diese Kräfte potentialtheoretisch berechnet werden. Im Allgemeinen kann nicht davon ausgegangen werden, dass Reibung vernachlässigt werden kann. In diesem Fall wäre die Potentialtheorie als alleiniges Vorhersagemodell unzureichend und müsste erweitert werden.

Für Kugeln als einfache Körper wurde die Strömung in periodisch instationären Strömungen über die Potentialtheorie hinaus bereits untersucht, jedoch nur für schleichende Strömungen, also für Strömungen in denen die Geschwindigkeiten und damit die Reynoldszahlen sehr klein sind. Diese kleinen Reynoldszahlen findet man in der Natur kaum. Interessant ist hingegen das Verhalten für größere Geschwindigkeiten und damit wesentlich größere Reynoldszahlen.

Im Jahr 1851 untersuchte Stokes erstmalig die Kräfte auf eine einfach geradlinig harmonisch oszillierende Kugel, einen Zylinder und eine unendlich langen Platte [3]. Er vernachlässigte die nichtlinearen Terme der Navier-Stokes-Gleichung und gab eine exakte Lösung für den instationären Fall an. Später untersuchten Basset [4], Boussinesq [5] und Oseen [6] die geradlinige Bewegung einer Kugel unter großer Beschleunigung in einem viskosen Fluid. Sie vernachlässigten ebenfalls die nichtlinearen Terme der Navier-StokesGleichung und stellten fest, dass die Kraft einer beschleunigten Kugel nicht nur von ihrer unmittelbaren Geschwindigkeit und Beschleunigung abhängt, also dem potentialtheoretischen Term und der Stokes'schen Reibungskraft, sondern zusätzlich auch von einem integralen Ausdruck, der Basset-Kraft, welche die zeitliche Änderung der Beschleunigung berücksichtigt. Die Kräfte auf oszillierende Kugeln für kleine Reynoldszahlen wurden bisher überwiegend numerisch untersucht [7-10]. Nur Odar und Hamilton untersuchten das 


\section{Einleitung}

Problem experimentell [11]. Sie modifizierten die Gleichung von Basset, Boussinesq und Oseen, indem sie einzelne Kraftterme mit experimentell bestimmten Faktoren gewichteten, um eine Aussage über die jeweiligen Kraftanteile treffen zu können. Für große Reynoldszahlen wurde die Gültigkeit der Gleichung von Basset, Boussinesq und Oseen hingegen noch nicht bestätigt.

Die Bestimmung von Kräften auf Körper in instationären Strömungsfeldern hat bei fahrenden Zügen große Bedeutung. Im Bezugssystem eines fahrenden Zuges ist die ihn umgebene Druckverteilung zeitlich und räumlich stationär. Eine sich im Bezugssystem relativ zum fahrenden Zug befindende Person wird diese Druckverteilung hingegen als instationäre Druckschwankung erfahren [12]. Durch diese Druckschwankungen werden wiederum aerodynamische Lasten auf die Person ausgeübt. Betroffene Personen können wartende Passagiere am Bahnsteig oder auch Gleisarbeiter auf offener Strecke sein. Da moderne Hochgeschwindigkeitszüge immer schneller fahren und dadurch die wirkende Kraft auf diese Personen größer wird, ist es umso wichtiger, die Sicherheit von Personen am Bahnsteig und an der Strecke bei Zugdurchfahrten weiterhin zu gewährleisten.

In einer europäischen Norm, der sogenannten „Technischen Spezifikation für die Interoperabilität (TSI)" sind unter anderem maximale Grenzwerte für die induzierten Geschwindigkeiten an bestimmten Positionen am Bahnsteig vorgegeben [13]. Wirkende Kräfte auf einen Körper in Abhängigkeit seiner Form, Größe oder des Abstandes zum Gleis werden hingegen nicht berücksichtigt, da es bisher unklar ist, welche physikalischen Effekte die Kräfte in instationären Strömungen, wie der Zugvorbeifahrt, dominieren. Dies soll durch Experimente geklärt werden.

Es ist jedoch nur bedingt möglich Messungen mit der benötigten Genauigkeit an realen Zügen durchzuführen. In einem skalierten Modellexperiment, können Messungen hingegen effektiver durchgeführt werden. Durch die Möglichkeit, eine große Anzahl an Versuchen durchführen zu können, ergibt sich eine bessere Genauigkeit, zudem kann im Experiment eine bessere Reproduzierbarkeit der Messungen durch gleichbleibende Umgebungsbedingungen (Temperatur, Wind usw.) erreicht werden. Des Weiteren lassen sich Versuchsumbauten, wie zum Beispiel die Anwendung unterschiedlicher Messtechniken oder der Wechsel des Untergrundes, einfacher realisieren. Daher wäre es für die Entwicklung von neuen Zuggeometrien günstig, wenn die aerodynamisch induzierten Geschwindigkeiten und wirkende Kräfte auf Körper in Gleisnähe durch einen vorbeifahrenden Zug bereits in der Entwurfsphase in Modellexperimenten überprüft werden könnten.

Das Strömungsfeld eines fahrenden Zuges kann in drei Bereiche eingeteilt werden: Die durch den Zugkopf hervorgerufene Druckwelle, genannt Kopfwelle, die Grenzschicht, welche sich neben dem Zug in Abhängigkeit der Länge des Zuges aufbaut und der Nachlauf hinter dem Zug [14,15]. Dieser Arbeit konzentriert sich auf die durch die Kopfwelle eines vorbeifahrenden Zuges induzierten Kräfte auf Kugeln und Scheiben. In geltenden Normen, wie der TSI-Norm [13], werden lediglich Geschwindigkeiten am Gleis berücksichtigt, 
jedoch nicht die Druckschwankung durch die Kopfwelle, welche besonders gefährlich sein kann, da durch sie Personen unter die Lok geraten können. Der Verlauf der Kopfwelle hängt stark von der Kopfform ab. Je schlanker die Kopfgeometrie, desto schwächer ausgeprägt ist die zeitliche Druckänderung [16]. Während der Vorbeifahrt des Zuges an einem Körper ist dieser starken Druckänderungen ausgesetzt. Vor dem Zugkopf herrscht ein Überdruck. Direkt nach dem Passieren der Zugnase herrscht jedoch starker Unterdruck $[12,16]$. Es besteht die Gefahr, dass Personen durch die transiente Kraft aus dem Gleichgewicht und unter die Lok geraten können [17]. Als Richtwert werden in etwa $100 \mathrm{~N}$ angegeben [12]. Allerdings ist nicht nur die Amplitude relevant, sondern auch die Schnelle der Druckwelle. Diese hängt, genau wie die Amplitude der Druckschwankung, maßgeblich von der Kopfgeometrie des Zuges ab [18]. Diese Auswirkungen der Kräfte auf Personen durch die Kopfwelle zeigen, dass lediglich eine Angabe einer maximalen Geschwindigkeit am Gleis, wie aktuell von der TSI-Norm vorgegeben, unzureichend für die Gefährdungsbeurteilung erscheint, da in der TSI-Norm weder die Zuggeometrie noch die Körperform noch der Einfluss der Druckschwankungen berücksichtigt werden.

Die Kopfwelle eines vorbeifahrenden Zuges lässt sich akkurat mit der Potentialtheorie beschreiben $[12,16]$. Bisher beschränkt sich die Literatur lediglich auf rotationssymmetrische Halbkörper-Modelle als Zuggeometrien [12,19]. Diese stellen heutige Konfigurationen nur unzureichend dar. Mithilfe dieses elementaren Modells des „räumlichen Halbkörpers" lässt sich abschätzen, welche Wirkung ein fahrender Zug auf einen neben dem Gleis befindlichen unendlich langen, unendlich dünnen Zylinder ausübt [12]. Daraus lassen sich bereits Sicherheitsvorschriften ableiten: zum Beispiel der von Gleisarbeitern beim Passieren eines Zuges einzuhaltende Mindestabstand vom Gleis oder der Sicherheitsabstand von wartenden Passagieren am Bahnsteig [16]. Es ist unklar, bei welchen Objekten weitere physikalische Effekte, wie beispielsweise Reibung, relevant werden und wo die Grenzen der Potentialtheorie liegen. Sie wäre dann in diesen Bereichen als Vorhersagemodell nicht mehr hinreichend genau und Gefahren können unterschätzt werden. Das Ziel dieser Arbeit ist die Ableitung eines einfachen analytischen Vorhersagemodells, welches die aerodynamischen Lasten auf einen Körper in instationären Strömungen, induziert durch einen vorbeifahrenden $\mathrm{Zug}$, genau genug vorhersagen kann.

Um dieses Ziel zu erreichen, wird zunächst zur Berechnung der Kräfte auf verschiedene Körper ein flexibles potentialtheoretisches Modell benutzt, welches nur eine sehr kurze Rechenzeit benötigt. Zur Überprüfung des potentialtheoretischen Modells werden in der Tunnel-Simulations-Anlage Göttingen (TSG) am Deutschen Zentrum für Luftund Raumfahrt (DLR) Geschwindigkeits- und Kraftmessungen durchgeführt. Im Anschluss werden zur Validierung der Gleichung von Basset, Boussinesq und Oseen die Kräfte auf eine Kugel in einem instationären Strömungsfeld für große Reynoldszahlen gemessen. Das Ergebnis dieser Messung wird abschließend verwendet, um das potentialtheoretische Vorhersagemodell für die induzierten Kräfte auf Kugeln durch einen vorbeifahrenden Zug zu optimieren. 


\section{Einleitung}

In Kapitel 2 werden die theoretischen Zusammenhänge erläutert, insbesondere die Herleitung des potentialtheoretischen Zugmodells und die Modellbildung der Körper. In den Kapiteln 3 bis 6 werden die durchgeführten Experimente an der TSG und die verwendete Messtechnik vorgestellt und gezeigt, ob und wie genau das potentialtheoretische Modell die Kräfte für verschiedene Körper vorhersagt. Es werden verschiedene Messmethoden miteinander verglichen, um auszuschließen, dass Abweichungen zwischen Theorie und Experiment durch die verwendete Messtechnik entstehen. In Kapitel 7 wird die Theorie von Basset, Boussinesq und Oseen vorgestellt, welche die bisher verwendeten potentialtheoretischen Terme zur Kraftberechnung um weitere Terme erweitert. Zur Überprüfung dieser Theorie werden Kraftmessungen auf Kugeln im instationären Strömungsfeld eines Lautsprechers gezeigt. Die dort erhaltenen Ergebnisse werden schließlich auf die Ergebnisse der Vorbeifahrt-Messungen in der TSG angewendet. In Kapitel 8 werden die Ergebnisse zusammengefasst und es wird ein Ausblick auf weitere Arbeiten gegeben. 
KAPITEL 2

\section{Analytische Berechnung der Zugvorbeifahrt mittels Potentialtheorie}

Das Strömungsfeld eines fahrenden Zuges kann in drei Bereiche eingeteilt werden: Die durch den Zugkopf hervorgerufene Druckwelle, genannt Kopfwelle, die Grenzschicht, welche sich neben dem Zug in Abhängigkeit der Länge des Zuges aufbaut und der Nachlauf hinter dem Zug [14,15]. Die Kopfwelle eines vorbeifahrenden Zuges kann näherungsweise mithilfe der Potentialtheorie berechnet werden [12,14,18-20]. Diese Arbeit konzentriert sich auf Kräfte auf Körper in instationären Strömungen, welche durch die Kopfwelle eines vorbeifahrenden Zuges induziert werden.

Um die Sicherheit von Passagieren und Gleisarbeitern bei Zugdurchfahrten zu gewährleisten, sind Richtlinien erforderlich, welche Kräfte hierbei maximal auf Personen in Gleisnähe wirken dürfen. Entsprechend werden in einer europäischen Norm, der so genannten „Technischen Spezifikation für die Interoperabilität (TSI)“, verschiedene Anforderungen an vorbeifahrende Züge gestellt [13]. Diese Anforderungen berücksichtigen allerdings nicht die wirkenden Kräfte, ausgelöst durch die Druckschwankungen der Kopfwelle des vorbeifahrenden Zuges auf einen Körper in Abhängigkeit seiner Größe oder seines Abstandes zum Gleis. Unter Anderem sind maximale Grenzwerte für die induzierten Geschwindigkeiten an bestimmten Positionen am Bahnsteig vorgegeben.

Die Druckschwankungen hingegen sind aber besonders gefährlich, da wartende Personen am Gleis aus dem Gleichgewicht geraten können [17,18]. Relevant ist nicht nur die Amplitude der Druckwelle, sondern auch die Schnelle. Die Schnelle hängt maßgeblich von der Kopfgeometrie des Zuges ab [18]. Lediglich eine Angabe einer maximalen, durch den vorbeifahrenden Zug induzierten Geschwindigkeit am Gleis scheint daher unzureichend, da diese weder die Zuggeometrie noch die Körperform berücksichtigt. Daher ist es wichtig, sich mit den aerodynamischen Lasten auf Körper in instationären Strömungen zu befassen. 


\section{Analytische Berechnung der Zugvorbeifahrt mittels Potentialtheorie}

In diesem Kapitel werden die Grundlagen für drehungsfreie, also reibungsfreie, inkompressible Strömungen erläutert (Abschnitt 2.1). Die analytischen Berechnungen einer schlanken (Abschnitt 2.2.1) und einer stumpfen Zugkontur (Abschnitt 2.2.1) als Superposition elementarer Potentiallösungen werden vorgestellt. Der Körper am Gleis wird ebenfalls mittels potentialtheoretischer Methoden beschrieben (Abschnitt 2.2.3). Schließlich wird der instationäre Fall der Vorbeifahrt eines Zuges an diesem Körper potentialtheoretisch hergeleitet und daraus die aerodynamischen Lasten auf den Körper bei der Vorbeifahrt berechnet (Abschnitt 2.3). Zur Untersuchung des Einflusses der Zuggeometrie werden die Kräfte auf eine Kugel durch die verschiedenen berechneten potentialtheoretischen Zuggeometrien miteinander verglichen (Abschnitt 2.4).

\subsection{Grundgleichungen der reibungsfreien Strömung}

Üblicherweise ist die mittlere freie Weglänge von Molekülen in der Strömung klein gegenüber charakteristischen geometrischen Abmessungen. Daher können Fluide als Kontinuum idealisiert werden, dessen makroskopische Eigenschaften stetige Funktionen des Ortes sind. Somit gilt die Hypothese, dass ein Fluid mit beliebig kleinem Volumen immer noch makroskopische Eigenschaften besitzt. Zur mathematischen Beschreibung des Fluids wird das Konzept des „Fluidteilchens“ eingeführt. Es ist definiert als ein kleines fluidgefülltes Volumen, welches die lokalen makroskopischen Eigenschaften besitzt. Es ist jedoch groß gegen die mittlere freie Weglänge der einzelenen Moleküle. Es kann also jedem Teilchen an einem Ort $\vec{r}=x \overrightarrow{e_{x}}+y \overrightarrow{e_{y}}+z \overrightarrow{e_{z}}$ zur Zeit $t$ eine Dichte $\rho(\vec{r}, t, T(\vec{r}))$, ein Druck $p(\vec{r}, t, T(\vec{r}))$ usw. bei einer Temperatur $T(\vec{r})$ zugeordnet werden. Das Fluidteilchen wird wiederum so klein gewählt, dass es als Punktteilchen im Sinne der Newtonschen Mechanik behandelt werden kann. Ihm wird also eine Geschwindigkeit $\vec{u}(\vec{r}, t)$ zugeordnet und es kann durch Kräfte $\vec{F}(\vec{r}, t)$ beschleunigt werden [21,22].

In reibungsfreier Strömung können keine Schubspannungen auf Fluidteilchen wirken, da die Viskosität und daher die Reibung als vernachlässigbar klein angesehen werden. Es sind also nur Druck und Massenkräfte vorhanden. Dies wird durch die Impulsgleichung für reibungsfreie Strömungen, der EuLER-Gleichung beschrieben [21]:

$$
\frac{\partial \vec{u}}{\partial t}+(\vec{u} \nabla) \vec{u}=-\frac{1}{\rho} \nabla p
$$

In einer drehungsfreien Strömung gilt

$$
\operatorname{rot} \vec{u}=0 .
$$

Es existiert wegen der allgemeinen Identität

$$
\operatorname{rot} \operatorname{grad} \phi=0,
$$

ein Geschwindigkeitspotential $\phi(x, y, z, t)$ mit $\vec{u}=\operatorname{grad} \phi$, den Raumkoordinaten $x, y$ und $z$ und der Zeit $t$. Daher kommt auch der Name „Potentialströmung“ [21]. 
Die Koordinaten sind so definiert, dass $x$ in Fahrtrichtung, $y$ quer zur Fahrtrichtung und $z$ nach oben zeigt. Ist das Fluid zudem noch inkompressibel, so folgt aus der Kontinuitätsgleichung

$$
\underbrace{\frac{D \rho}{D t}}_{=0}+\rho \operatorname{div} \vec{u}=0
$$

und aus div grad $\phi=\Delta \phi$ sofort die LAPLACE-Gleichung [21]:

$$
\Delta \phi=0 .
$$

Jede Funktion $\phi$, für die Gleichung 2.5 gilt, kann, unter Erfüllung der Randbedingungen als Geschwindigkeitspotential einer Potentialströmung aufgefasst werden. Diese können sein, dass feste Wände nicht durchströmt werden können, also die Normalgeschwindigkeit $\partial \phi / \partial n$ verschwinden muss. In Potentialströmungen existiert keine Wandhaftung, weshalb gilt: $\vec{u} \cdot \vec{n} \stackrel{!}{=} 0$ mit $\vec{n}$ als Normalenvektor der Wand [21].

\subsection{Superposition elementarer Potentiallösungen: Stromlinienkörper}

Mit der Einführung eines Geschwindigkeitspotentials ist es möglich, durch die Verwendung verschiedener Funktionen, beliebig viele Strömungsfelder zu erhalten. Auch Körperkonturen sind möglich, da jede Stromlinie auch wieder als Wand interpretierbar ist. Zudem ist die Feldgleichung der skalaren Funktion $\phi(x, y, z, t)$ linear [21], so dass auch jede Linearkombination von Gleichungen die Laplace-Gleichung löst und damit wieder ein Geschwindigkeitspotential darstellt.

So kann beispielsweise ein geschlossener Strömungskörper durch die Superposition einer ebenen Strömung mit einer Quellen-/Senkenströmung dargestellt werden. Mithilfe der Superposition elementarer Potentiallösungen können verschiedene Stromlinienkörper erzeugt werden, zum Beispiel Schiffe [23] oder Züge [12]. Nähern sich eine Quelle und eine Senke an, so dass der Abstand zwischen beiden gegen Null geht, erhält man eine Dipolverteilung. Wird diese mit einer ebenen Potentialströmung überlagert, erhält man einen Kreiszylinder, bzw. eine Kugel im dreidimensionalen Strömungsfeld [21].

\subsubsection{Schlanke Zuggeometrie}

Zur Validierung der Potentialtheorie als Vorhersagemethode für Kräfte auf Objekte in Gleisnähe werden Modellexperimente in der Tunnel-Simulations-Anlage Göttingen (TSG) durchgeführt (siehe Kapitel 6). Um möglichst die gleichen Voraussetzungen in der Theorie und dem Experiment gewährleisten zu können, soll die Geometrie des potentialtheoretischen Modells an das existierende ICE3-Modell angenähert werden. Der hier analytisch berechnete Potentialzug wird ebenfalls als Modell im Maßstab 1:25 konstruiert. Der Potentialzug wird als Haube konstruiert und muss über einen $l=2,10 \mathrm{~m}$ langen Zugträger passen, der in der TSG fahren kann (siehe Abbildung 2.2). 


\section{Analytische Berechnung der Zugvorbeifahrt mittels Potentialtheorie}

In dieser Arbeit wird eine generische Zuggeometrie mit der Querschnittfläche $A$ als RANKINE-Körper in einer ebenen Potentialströmung $u_{\infty}$ in $x$-Richtung modelliert. In der Literatur wurden bisher der Einfachheit halber rotationssymmetrische Einpunktquellen als Zuggeometrie verwendet und damit häufig zweidimensional gerechnet [12, 14, 18, 20]. Zur besseren Genauigkeit und Vergleichbarkeit mit den Modellexperimenten wird hier die Zuggeometrie durch eine dreidimensionale Quellen-/Senkenströmung dargestellt. Das Geschwindigkeitspotential $\phi$ als Lösung der Laplace-Gleichung (2.5) einer solchen Strömung lautet wie folgt:

$$
\phi_{Z u g}=u_{\infty} x-\sum_{i}^{n} \frac{Q_{i}}{4 \pi} \frac{1}{\sqrt{\left(x-x_{i}\right)^{2}+\left(y-y_{i}\right)^{2}+\left(z-z_{i}\right)^{2}}} .
$$

Hierbei handelt es sich bei $Q_{i}$ um die Stärken der Quellen bzw. Senken und bei $x_{i}, y_{i}$ und $z_{i}$ um ihre Positionen. Die Quellstärke entspricht dem Durchfluss durch den Körper:

$$
Q^{*}=\sum_{i=1}^{k} Q_{i}=A \cdot u_{\infty},
$$

für $k$ Quellen im Zugkopf. Der Zugquerschnitt $A=0,016 \mathrm{~m}^{2}$ entspricht in etwa dem des ICE3-Modells (siehe Tabelle 2.1). Da es sich hierbei um ein 1:25 Modell handelt, entspricht $A$ einer Querschnittsfläche von $A^{\prime}=10 \mathrm{~m}^{2}$ im Großmaßstab. Die Quellpositionen $x_{i}, y_{i}$ und $z_{i}$ können Tabelle 2.2 entnommen werden. $u_{\infty}$ ist die Fahrt- bzw. Anströmgeschwindigkeit.

\begin{tabular}{c|c|c|c} 
Modell & Höhe $[\mathrm{cm}]$ & Breite $[\mathrm{cm}]$ & $A\left[\mathrm{~cm}^{2}\right]$ \\
\hline ICE3 & 14,5 & 11,80 & 155 \\
Potentialzug & 14,4 & 13,81 & 160
\end{tabular}

Tabelle 2.1.: Vergleich der Geometrien des ICE3-Modells und des berechneten Potentialzugs im Maßstab 1:25.

Durch Variation der Quellpositionen und -anzahl können verschiedene Geometrien realisiert werden. In dieser Arbeit wird der Zugkopf durch $k=3$ in der $x-z$-Ebene äquidistante, kollinear angeordnete Quellen realisiert. Entsprechende, an der $y-z-$ Ebene gespiegelten Senken bilden das Heck. Die Quellpositionen werden manuell iterativ ermittelt, so dass der Potentialzug als Haube für die späteren Experimente auf den Zugträger mit einer Länge von $l=2,1 \mathrm{~m}$ passt. Die Quellen dürfen auch nicht zu weit auseinander liegen, da die Kontur sonst „eingedellt“ wird (siehe Abbildung 2.1). In Abschnitt 6.2 wird ein automatisiertes Iterationsverfahren vorgestellt, welches die Quellpositionen und damit die Zugkopfgeometrie anhand experimenteller Ergebnisse optimiert. In Abbildung 2.2 sind die Konturen des ICE3 Modells des Potentialzugs mit Zugträger und Quellpositionen skizziert. 


\begin{tabular}{ccc|c}
$x_{i}[\mathrm{~m}]$ & $y_{i}[\mathrm{~m}]$ & $z_{i}[\mathrm{~m}]$ & $Q_{i} /\left(A \cdot u_{\infty}\right)$ \\
\hline 0,000 & 0,0 & 0,00 & $1 / 3$ \\
0,025 & 0,0 & 0,02 & $1 / 3$ \\
0,050 & 0,0 & 0,04 & $1 / 3$ \\
2,150 & 0,0 & 0,04 & $-1 / 3$ \\
2,175 & 0,0 & 0,02 & $-1 / 3$ \\
2,200 & 0,0 & 0,00 & $-1 / 3$
\end{tabular}

Tabelle 2.2.: Positionen der Quellen und Senken und ihre Quellstärken.

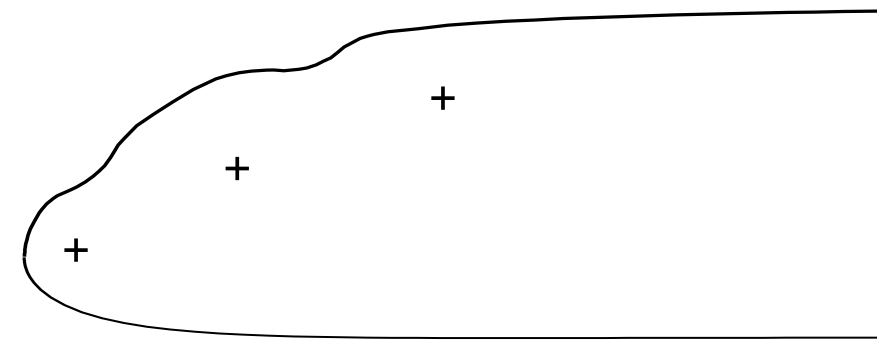

Abbildung 2.1.: Beispiel für eine Kontur mit zu weit auseinander liegenden Quellen (schematische Darstellung).

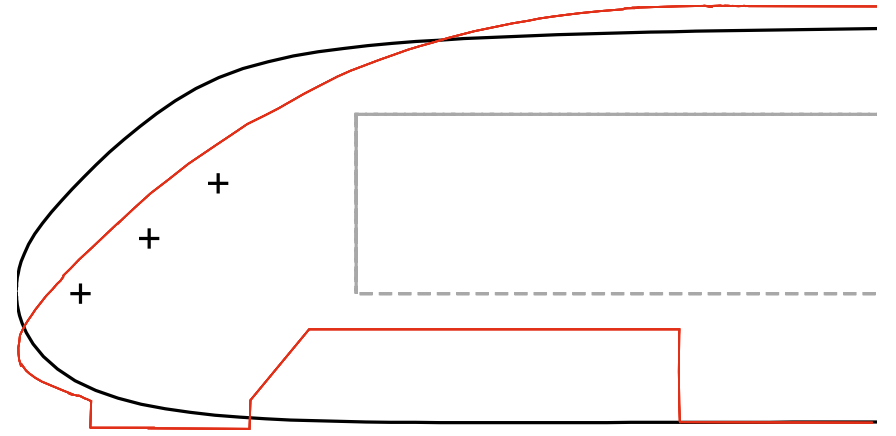

Abbildung 2.2.: Vergleich der Konturen des Potentialszugs (schwarz) mit Quellpositionen und Trägerbalken (grau) und des ICE3 (rot). 


\section{Analytische Berechnung der Zugvorbeifahrt mittels Potentialtheorie}

Für die Simulation des Bodens werden die Quellen und Senken in der Ebene $z=-b / 2$ gespiegelt, mit $b=0,1275 \mathrm{~m}$, so dass ein Spiegelzug entsteht. Es gibt, bei dem hier berechneten $\mathrm{Zug}$, insgesamt (mit gespiegeltem Zug) $n=4 \cdot k=12$ Quellen. Der Wert für $b$ wurde so gewählt, dass der Abstand zwischen Zugboden und Schienenoberkante ca. 3 $\mathrm{cm}$ entspricht, welches auch der Abstand beim ICE3-Modell ist, damit auch hier eine Ähnlichkeit der beiden Zugmodelle gewährleistet ist.

Es kann mit drei Quellen nicht die exakte Geometrie des ICE3 nachgebildet werden, da es bei einer so geringen Quellenanzahl nicht möglich ist, die Drehgestelle, die Stromabnehmer oder die Übergänge zwischen den einzelnen Wagen darzustellen. Die Quellen wurden jedoch so gewählt, dass die Geometrien zumindest ähnlich sind. Die genauen Geometriedaten des potentialtheoretischen Zugmodells im Vergleich zum ICE3 können Tabelle 2.1 entnommen werden.

Für die Darstellung der Zugkontur müssen die Stromlinien berechnet werden. Stromlinien sind ein geometrisches Hilfsmittel zur anschaulichen Beschreibung einer Strömung. Sie sind so definiert, dass ihre Tangentenrichtung mit der Richtung der Geschwindigkeitsvektoren zu einem bestimmten Zeitpunkt übereinstimmt. Diese werden aus den Geschwindigkeiten $u, v$ und $w$ in $x$-, $y$ - und $z$-Richtung berechnet, welche der partiellen Ableitung des Potentials nach der jeweiligen Raumrichtung entspricht. Die Ableitungen lauten wie folgt:

$$
\begin{aligned}
& u_{Z u g}=\frac{\partial \phi_{Z u g}}{\partial x}=\sum_{i}^{n} \frac{Q_{i}}{4 \pi} \cdot \frac{x-x_{i}}{\left(\left(x-x_{i}\right)^{2}+\left(\left(y-y_{i}\right)^{2}\left(\left(z-z_{i}\right)^{2}\right)^{3 / 2}\right.\right.} \\
& v_{Z u g}=\frac{\partial \phi_{Z u g}}{\partial y}=\sum_{i}^{n} \frac{Q_{i}}{4 \pi} \cdot \frac{y-y_{i}}{\left(\left(x-x_{i}\right)^{2}+\left(\left(y-y_{i}\right)^{2}\left(\left(z-z_{i}\right)^{2}\right)^{3 / 2}\right.\right.} \\
& w_{Z u g}=\frac{\partial \phi_{Z u g}}{\partial z}=\sum_{i}^{n} \frac{Q_{i}}{4 \pi} \cdot \frac{z-z_{i}}{\left(\left(z-z_{i}\right)^{2}+\left(\left(y-y_{i}\right)^{2}\left(\left(z-z_{i}\right)^{2}\right)^{3 / 2}\right.\right.}
\end{aligned}
$$

Mit (2.8-2.10) kann die Geschwindigkeit eines Fluidteilchens an jedem Ort im Strömungsfeld bestimmt werden. Der Punkt auf der Oberfläche des Körpers, an dem die Strömungsgeschwindigkeit gleich Null ist, wird „Staupunkt" genannt. Um ihn herum zerteilen sich die Stromlinien, um den Körper zu umströmen. Diejenige Stromlinie, die als einzige im Staupunkt endet, wird „Staupunktsstromlinie“ genannt [21].

Um den Staupunkt zu finden, wird der „Predictor-Corrector-Algorithmus“ verwendet [24]. Dazu wird genügend weit vor der ersten Quelle gestartet (hier bei $x=-1 \cdot 10^{-4} \mathrm{~m}$ ). In einer vorgegebenen Schrittweite von $d x=1 \cdot 10^{-6} \mathrm{~m}$ wird vom Startpunkt in $x, y$ und $z$ Richtung um den Wert $1,1 \cdot d x$ vorangegangen und $|\vec{u}|^{2}$ berechnet. Ist der neue Wert für $|\vec{u}|^{2}$ kleiner als der vorherige, wird dieser Wert als neuer Wert $|\vec{u}|^{2}$ gespeichert und weiter um $1,1 \cdot d x$ vorangegangen. Andernfalls wird um $0,5 \cdot d x$ zurückgegangen. Dies 
geschieht für alle Raumrichtungen so lange, bis $|\vec{u}|^{2}=u^{2}+v^{2}+w^{2} \approx 0$ erfüllt ist. In diesem Fall bei einer Anströmgeschwindigkeit von $u_{\infty}=40 \mathrm{~m} / \mathrm{s}$ befindet sich der Staupunkt bei $\vec{x}_{S P}(x, y, z)=(-0,0232 ; 0,0 ; 0,00102) \mathrm{m}$.

Zur Bestimmung der Staupunktsstromlinie, welche die Körperkontur ergibt, wird die Geschwindigkeitsberechnung entlang einer Stromlinie in einem kleinen Kreis $(R=$ $4,5 \cdot 10^{-5} \mathrm{~m}$ ) um den Staupunkt herum gestartet, da sonst kein Gradient im Raum von $\phi$ gebildet werden kann. Die Körperkontur ergibt sich schließlich aus der Berechnung der Geschwindigkeiten (Gleichungen 2.8-2.10) in einer Schrittweite von $d x=u_{\infty} \cdot d t$ mit $d t=10^{-7} \mathrm{~s}$. Abbildung 2.3 zeigt den Potentialkörper mit seinem Spiegelzug, den daraus resultierenden Boden und den Stromlinien als Schnitt durch die x-z-Ebene. Abbildung 2.4 zeigt die dreidimensionale Darstellung des Potentialzugs.

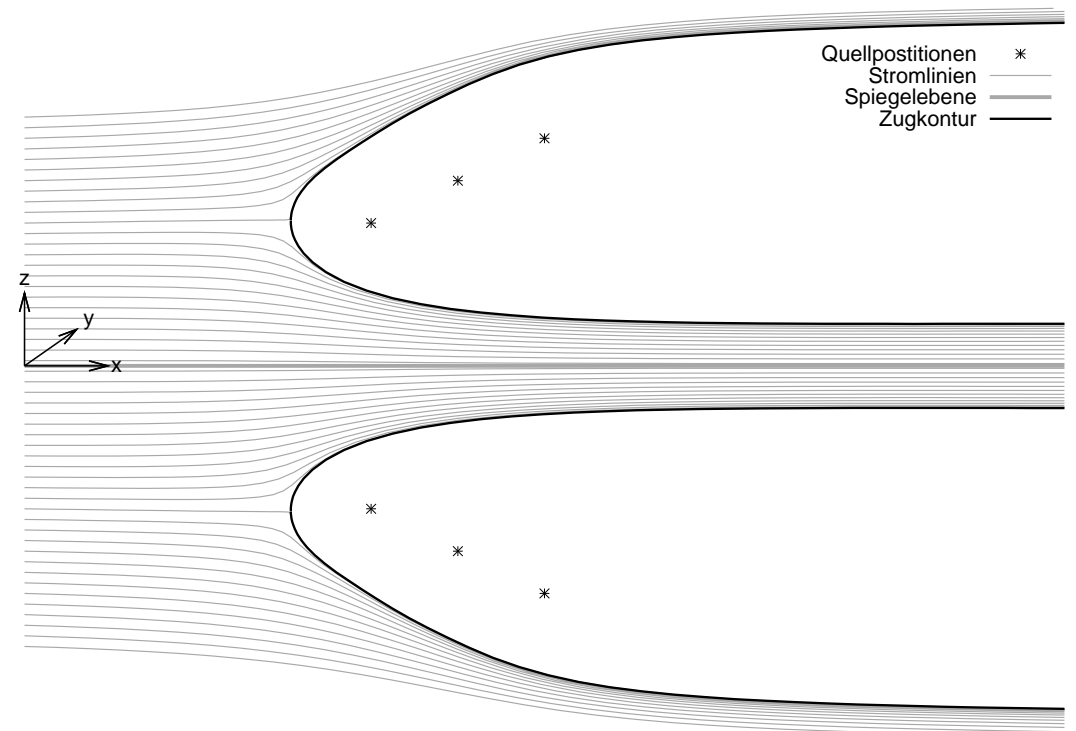

Abbildung 2.3.: Potentialkörper mit Spiegelzug, Quellen und Senken und den daraus resultierenden Stromlinien, Schnitt durch die x-z-Ebene.

Damit das Potentialzugmodell in der TSG bei den späteren Experimenten nicht abhebt, darf es keinen Auftrieb haben. Das heißt, dass die Kraft in $z$-Richtung negativ sein muss. Dazu wird der Druck auf der Zugoberfläche berechnet und über die gesamten Oberfläche integriert. Als Ergebnis erhält man $F_{z}=-7,37 \cdot 10^{-2} \mathrm{~N}$, das heißt der Zug hat einen Abtrieb. Abbildung 2.5 zeigt die $c_{p}$-Verteilung am Zugkopf. 


\section{Analytische Berechnung der Zugvorbeifahrt mittels Potentialtheorie}

Der Druckbeiwert wird dabei wie folgt berechnet:

$$
c_{p}=1-\frac{\left|\vec{u}^{2}\right|}{u_{\infty}^{2}} .
$$

Auf diesem Bild ist bereits zu erkennen, dass direkt an der Zugnase ein Überdruckgebiet herrscht und kurz hinter der Nase ein Unterdruckgebiet. Das heißt, ohne die Kraft auf ein Objekt berechnet zu haben, ist am bloßen Druckfeld des Zuges schon zu erkennen, dass ein Objekt neben dem Zug erheblichen Druckschwankungen ausgesetzt sein wird, wenn der Zug an ihm vorbeifährt.

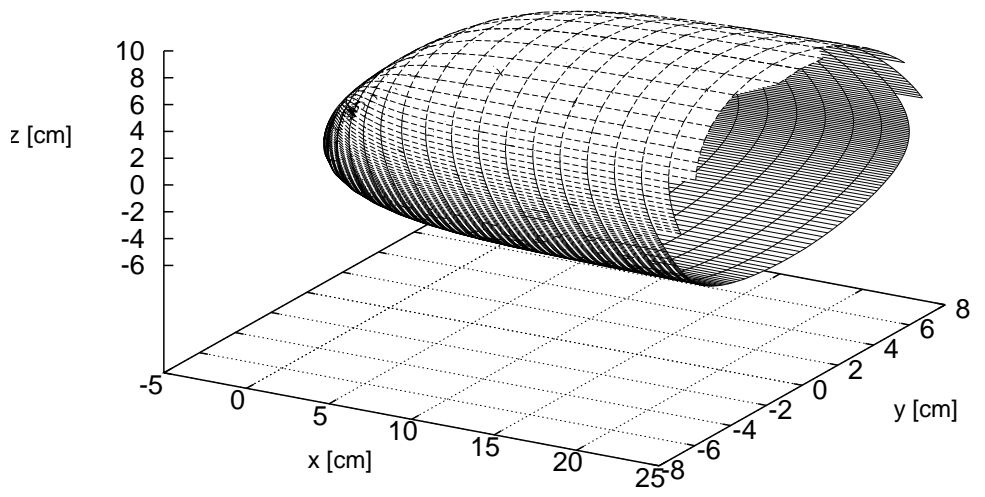

Abbildung 2.4.: 3D-Kontur des Potentialzugs.

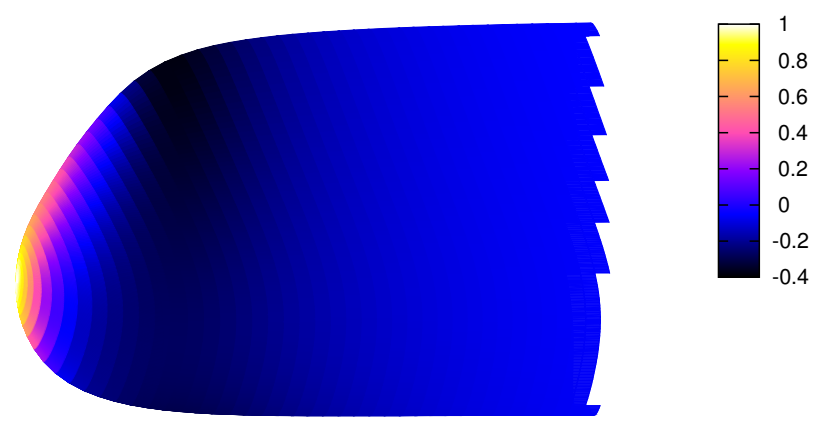

Abbildung 2.5.: Dreidimensionale Darstellung des Potentialzugkopfs. Die farbige Skala gibt den $c_{p}$-Wert auf der Oberfläche an. 


\subsubsection{Stumpfe Zuggeometrie}

Im vorigen Kapitel wurde gezeigt, dass sich schlanke Zuggeometrien gut mithilfe von Superposition von Elementarlösungen der Laplace-Gleichung darstellen lassen. Allerdings haben insbesondere Güterzüge oder die Baureihe E120 der Deutschen Bahn eher kastenförmige Lokomotivbugformen. In [1] wurde eine Methode entwickwelt, wie auch solche Zuggeometrien potentialtheoretisch berechnet werden können. Hierbei wird statt dem Geschwindigkeitsfeld mehrerer Punktquellen, wie im vorherigen Abschnitt, das Geschwindigkeitsfeld einer rechteckigen Quellverteilung mit konstanter Quellstärke $q_{0}$ berechnet und ebenfalls mit einer ebenen Potentialströmung $u_{\infty}$ superponiert. Die dadurch entstehende Verdrängungsströmung bildet die Zugkontur. Der Zug wird am Boden bei $z=0$ gespiegelt (siehe Abbildung 2.6).

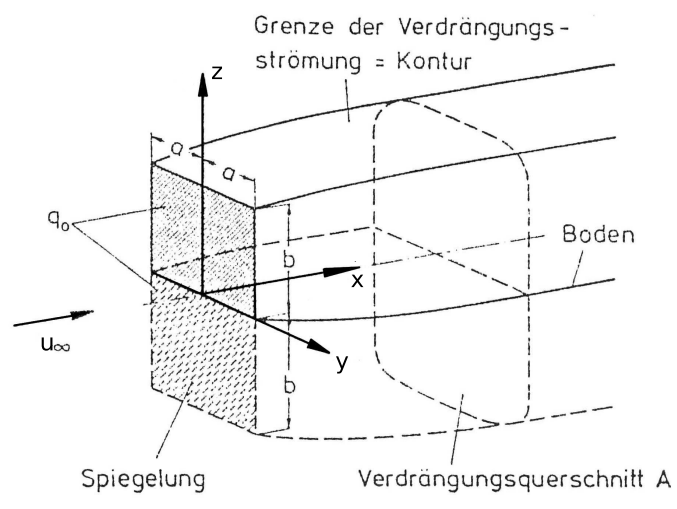

Abbildung 2.6.: Darstellung des entstehenden Strömungsfeldes durch eine rechteckige Quellenverteilung (aus [1], bearbeitet).

Die Geschwindigkeit senkrecht auf einer ebenen Quellfläche muss $u_{q_{0}}=\frac{q_{0}}{2}$ sein, da nach beiden Richtungen gleich viel ausströmt. $u_{q_{0}}$ nimmt mit abnehmender Entfernung $x$ zur Quellfläche zu und erreicht gerade den Wert $-u_{\infty}$, so dass die Anströmung $u_{\infty}$ $\mathrm{zu} u_{q_{0}}=0$ aufgestaut wird. Dies ist gerade der Staupunkt. Die rechteckige Quellfläche gehört daher zur Körperkontur der Verdrängungsströmung. In diesem Fall ergibt sich für den Gesamtvolumenstrom $Q^{*}$ der Quellfläche [1]:

$$
Q^{*}=q_{0} \cdot 2 a \cdot 2 b=8 u_{\infty} \cdot a \cdot b .
$$

Für große positive $x$ ergibt sich die Querschnittsfläche:

$$
A=8 \cdot a \cdot b
$$

und für die induzierten Geschwindigkeiten gilt [1]:

$$
u_{Z u g}=\frac{q_{0}}{4 \pi}[f(\alpha, \beta)-f(\bar{\alpha}, \beta)-f(\alpha, \bar{\beta})+f(\bar{\alpha}, \bar{\beta})],
$$




\section{Analytische Berechnung der Zugvorbeifahrt mittels Potentialtheorie}

mit

$$
f(\alpha, \beta)=\arctan \frac{\alpha \cdot \beta}{x \cdot \sqrt{\alpha^{2}+\beta^{2}+x^{2}}} .
$$

Dabei wurden folgende Abkürzungen verwendet:

$$
\alpha=y+a ; \quad \bar{\alpha}=y-a ; \quad \beta=z+b ; \quad \bar{\beta}=y-b .
$$

Für die Geschwindigkeiten in $y$ - und $z$-Richtung wird in Gleichung 2.14 für $v_{Z u g}$ statt $f(\alpha, \beta)$

$$
g(\alpha, \beta)=-\operatorname{arcsinh} \frac{\alpha}{\sqrt{\alpha^{2}+x^{2}}}
$$

eingesetzt. Für $w_{Z u g}$ wird entsprechend

$$
h(\alpha, \beta)=-\operatorname{arcsinh} \frac{\beta}{\sqrt{\alpha^{2}+x^{2}}}
$$

benutzt. Die Stromlinien starten an den Kanten der rechteckigen Quellfläche. Abbildung 2.7 zeigt die Stromlinien und die Quellfäche eines Kastenzuges mit $a=0,04 \mathrm{~m}$ und $b=0,05 \mathrm{~m}$, so dass mit Gleichung (2.13) eine Querschnittsfläche von $A=0,016 \mathrm{~m}^{2}$ entsteht. Möchte man also das Strömungsfeld eines Kastenzugs berechnen, werden statt der Gleichungen (2.8-2.10), die Gleichungen (2.14, 2.17 und 2.18) verwendet.

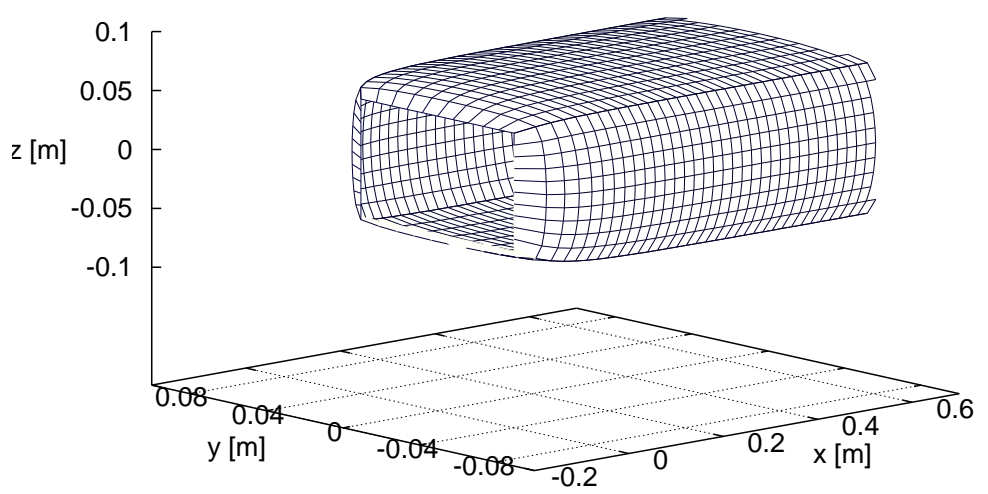

Abbildung 2.7.: Berechnete Kontur für einen Kastenzug mit $a=4 \mathrm{~cm}, b=5 \mathrm{~cm}$ und $A=0,016 \mathrm{~m}^{2}$. 


\subsubsection{Kugel}

In den vorherigen Abschnitten wurden die Strömungsfelder der potentialtheoretischen Zugkonturen berechnet. Im folgenden Abschnitt wird ein Objekt am Gleis, auf welches die Kraft des vorbeifahrenden Zuges wirken soll, berechnet. Zunächst handelt es sich bei dem Objekt um eine Kugel. Diese kann näherungsweise einen Menschen oder ein Gegenstand darstellen, welcher sich neben den Gleisen befindet. Die Kugel wird ebenfalls durch ein Geschwindigkeitspotential als Lösung der Laplace-Gleichung analytisch berechnet. Im Mittelpunkt der Kugel befindet sich ein dreidimensionaler Dipol. Durch die Überlagerung mit der Außenströmung $u_{\infty}$ und dem Strömungsfeld des Zuges entspricht die Verdrängungskontur der einer Kugel [21]. Das dreidimensionale Geschwindigkeitspotential des Dipols ergibt sich aus der Ableitung eines Monopols nach den drei Raumrichtungen. Das Potential des Monopols lautet:

$$
\phi_{M}=\frac{1}{4 \pi} \frac{Q_{M}}{r},
$$

mit $Q_{M}$ als Quellstärke des Monopols und $r=\sqrt{\left(x-x_{M}\right)^{2}+\left(y-y_{M}\right)^{2}+\left(z-z_{M}\right)^{2}}$. Bei $x_{M}, y_{M}$ und $z_{M}$ handelt es sich um die Koordinaten des Mittelpunktes der Kugel. Die Ableitungen lauten wie folgt:

$$
\begin{gathered}
\phi_{D, x}=\frac{\partial \phi_{M}}{\partial x}=-\frac{Q_{x}\left(x-x_{M}\right)}{r^{3}} \\
\phi_{D, y}=\frac{\partial \phi_{M}}{\partial y}=-\frac{Q_{y}\left(y-y_{M}\right)}{r^{3}} \\
\phi_{D, z}=\frac{\partial \phi_{M}}{\partial z}=-\frac{Q_{z}\left(z-z_{M}\right)}{r^{3}},
\end{gathered}
$$

so dass sich für den 3D-Dipol das folgende Potential ergibt:

$$
\phi_{D}=-\frac{1}{4 \pi}\left(\frac{Q_{x}\left(x-x_{M}\right)}{r^{3}}-\frac{Q_{y}\left(y-y_{M}\right)}{r^{3}}-\frac{Q_{z}\left(z-z_{M}\right)}{r^{3}}\right) .
$$

Die Quellstärken müssen so gewählt werden, dass die Oberfläche der Kugel nicht durchströmt wird. Dazu werden sechs Testpunkte auf der Oberfläche so gewählt, dass sie jeweils in der Raumrichtung des jeweiligen Dipols liegen. Das heißt, die drei Testpunkte in positiver Raumrichtung sind die Folgenden:

$$
\begin{aligned}
& \vec{T}_{1}(t)=R \cdot\left(\begin{array}{lll}
1 & 0 & 0
\end{array}\right)+\vec{x}_{D}(t) \\
& \vec{T}_{2}(t)=R \cdot\left(\begin{array}{lll}
0 & 1 & 0
\end{array}\right)+\vec{x}_{D}(t) \\
& \vec{T}_{3}(t)=R \cdot\left(\begin{array}{lll}
0 & 0 & 1
\end{array}\right)+\vec{x}_{D}(t)
\end{aligned}
$$

und jeweils die drei diametral gegenüberliegenden Punkte. Bei $R$ handelt es sich um den Kugelradius. Da sich die Kugel später quasistatisch am Zug vorbeibewegt (siehe Abschnitt 2.3), kommt der Vektor für die Kugelbewegung $\vec{x}_{D}(t)$ hinzu.

Damit die Kugeloberfläche nicht durchströmt wird, muss gelten:

$$
\left.\frac{\partial \phi_{D}}{\partial x}\right|_{\left(\begin{array}{lll}
R & 0 & 0
\end{array}\right)}+\left.\frac{\partial \phi_{Z u g}}{\partial x}\right|_{\left(\begin{array}{lll}
R & 0 & 0
\end{array}\right)}=0
$$




\section{Analytische Berechnung der Zugvorbeifahrt mittels Potentialtheorie}

Dies gilt auch für die anderen beiden Raumrichtungen. Da es sechs Testpunkte, aber nur drei Raumrichtungen gibt, ist das Gleichungssystem überbestimmt. Es wird daher folgendermaßen gelöst:

$$
\begin{aligned}
& \frac{1}{2}\left\{\left.\frac{\partial \phi_{Z u g}}{\partial x}\right|_{\left(\begin{array}{lll}
R & 0 & 0
\end{array}\right)}+\left.\frac{\partial \phi_{Z u g}}{\partial x}\right|_{\left(\begin{array}{lll}
-R & 0 & 0
\end{array}\right)}\right\}=\frac{1}{2}\left\{\frac{Q_{x 1}}{2 \pi R^{3}}+\frac{Q_{x 2}}{2 \pi R^{3}}\right\}
\end{aligned}
$$

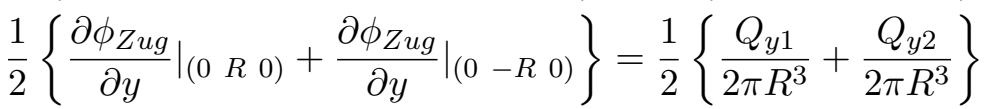

$$
\begin{aligned}
& \left.\frac{1}{2}\left\{\left.\frac{\partial \phi_{Z u g}}{\partial z}\right|_{(00 R)}+\left.\frac{\partial \phi_{Z u g}}{\partial z}\right|_{(0} 0-R\right)\right\}=\frac{1}{2}\left\{\frac{Q_{z 1}}{2 \pi R^{3}}+\frac{Q_{z 2}}{2 \pi R^{3}}\right\}
\end{aligned}
$$

Die Ableitungen des Potentials (2.8-2.10) sind bekannt und können mithilfe der obigen Formeln die Quellstärken des Dipols zu jedem Zeitpunkt bestimmt werden. Der Dipol wird, ebenso wie der Zug, an der Ebene $z=-b / 2$ gespiegelt.

\subsection{Simulation der Vorbeifahrt und daraus resultierende Kraft auf Objekte}

\subsubsection{Kraft auf eine Kugel}

Die Kraft auf die Kugel bei der Vorbeifahrt wird durch Integration des Drucks über die Kugeloberfläche bestimmt. Da reibungsfrei gerechnet wird, ist die zugrunde liegende Gleichung die EuLER-Gleichung (2.1). $\frac{\partial}{\partial t}(\operatorname{grad}) \phi$ eingesetzt in 2.1 ergibt

$$
\operatorname{grad}\left[\frac{\partial \phi}{\partial t}+\rho \frac{\vec{u}^{2}}{2}+\frac{p}{\rho}\right]=0 .
$$

Die Integration entlang einer Stromlinie ergibt schließlich die instationäre BERNOULLIGleichung:

$$
\frac{\partial \phi}{\partial t}+\frac{\vec{u}^{2}}{2}+\frac{p}{\rho}=\text { const. }=\frac{p_{\infty}}{\rho_{\infty}} .
$$

Hierbei ist $\phi$ das Gesamtpotential bestehend aus Dipol und Zug:

$$
\phi=\phi_{Z u g}+\phi_{D}
$$

und $\vec{u}^{2}$ das Quadrat der Summe der Geschwindigkeiten des Strömungsfeldes des Dipols und das des Zuges (inklusive ihrer Spiegelfelder)

$$
\vec{u}^{2}=\left(u_{Z u g}+\overrightarrow{u_{D}}\right)^{2}=\left(u_{Z u g}+u_{D}\right)^{2}+\left(v_{Z u g}+v_{D}\right)^{2}+\left(w_{Z u g}+w_{D}\right)^{2} .
$$

\section{Stationärer Fall}

Um den Druck mit der Bernoulli-Gleichung $2.26 \mathrm{zu}$ bestimmen, wird die Summe der Geschwindigkeitsfelder des Zuges (2.8-2.10) und des Dipols benötigt und mithilfe von 
Gleichung 2.28 zunächst der stationäre Anteil $\vec{u}^{2}$ bestimmt. Das Geschwindigkeitsfeld des Dipols ergibt sich ebenfalls wieder aus den Ableitungen des Dipolpotentials nach den drei Raumrichtungen:

$$
\begin{aligned}
& u_{D}=\frac{1}{4 \pi}\left(\frac{3 Q_{x}\left(x-x_{M}\right)^{2}}{r^{5}}-\frac{Q_{x}}{r^{3}}+\frac{3 Q_{y}\left(x-x_{M}\right)\left(y-y_{M}\right)}{r^{5}}+\frac{3 Q_{z}\left(x-x_{M}\right)\left(z-z_{M}\right)}{r^{5}}\right) \\
& v_{D}=\frac{1}{4 \pi}\left(\frac{3 Q_{y}\left(y-y_{M}\right)^{2}}{r^{5}}-\frac{Q_{y}}{r^{3}}+\frac{3 Q_{x}\left(x-x_{M}\right)\left(y-y_{M}\right)}{r^{5}}+\frac{3 Q_{z}\left(y-y_{M}\right)\left(z-z_{M}\right)}{r^{5}}\right) \\
& w_{D}=\frac{1}{4 \pi}\left(\frac{3 Q_{z}\left(z-z_{M}\right)^{2}}{r^{5}}-\frac{Q_{z}}{r^{3}}+\frac{3 Q_{x}\left(x-x_{M}\right)\left(z-z_{M}\right)}{r^{5}}+\frac{3 Q_{y}\left(y-y_{M}\right)\left(z-z_{M}\right)}{r^{5}}\right)
\end{aligned}
$$

\section{Instationärer Fall}

Für den instationären Fall der Vorbeifahrt wird der instationäre Anteil $\frac{\partial \phi}{\partial t}$ benötigt. Dazu wird die Kugel quasistatisch am Zug vorbei bewegt und so für jede Position der Kugel der Differenzenquotient des Potentials an zwei aufeinander folgenden Positionen des Zugs $d x=d t \cdot u_{\infty}$ bestimmt

$$
\frac{\partial \phi}{\partial t}=\frac{\phi\left(t_{1}-d t\right)-\phi\left(t_{1}\right)}{d t}
$$

Der Druck kann nun mithilfe der instationären Bernoulli-Gleichung (2.26) an jedem Punkt auf der Kugeloberfläche bestimmt werden. Die Integration des Drucks über die gesamte Kugeloberfläche ergibt die Kraft auf die Kugel in $x$-, $y$ - und $z$-Richtung zu einem Zeitpunkt $t$ :

$$
\vec{F}=\int p \cdot \vec{n} d S
$$

Die Kugel wird weiter quasistatisch am Zug vorbei bewegt und zu jedem Zeitpunkt die Kraft berechnet, so dass sich ein Kraftverlauf über die Kugelposition neben dem Zug ergibt. Abbildung 2.8 zeigt die Vorbeifahrt von oben bei $x=t \cdot u_{\infty}=0$.

Die instationäre Bernoulli-Gleichung (2.26) besteht aus zwei Anteilen: dem instationären Anteil $\frac{\partial \phi}{\partial t}$ und dem stationären Anteil $\frac{\vec{u}^{2}}{2}$. Für Abbildung 2.9 sind beide Anteile getrennt simuliert worden. Bei $x=0 \mathrm{~m}$ befinden sich Zugnase und der Mittelpunkt der Kugel an der selben Position. Bei $c_{F}=F /\left(0,5 \rho_{0} u_{\infty}^{2} A\right)$ handelt es sich um den Kraftbeiwert in $y$-Richtung und $D=12 \mathrm{~cm}$ ist eine übliche Zugbreite. Die Kurven zeigen, dass sich der Kraftvektor bei der Vorbeifahrt des Zugkopfes fast komplett dreht. Zunächst wird das Objekt vom Zug weggedrückt. Sobald die Nase vorbei ist, wird es jedoch in Richtung des Zuges gezogen. Es ist zu erkennen, dass der stationäre Anteil nur maximal $10 \%$ zur Gesamtkraft beiträgt. Der Hauptanteil an der Kraft ist also der instationäre Anteil. Es genügt daher nicht, die Kraft auf die Kugel nur stationär zu berechnen, da der instationäre Anteil eine so relevante Rolle spielt. 


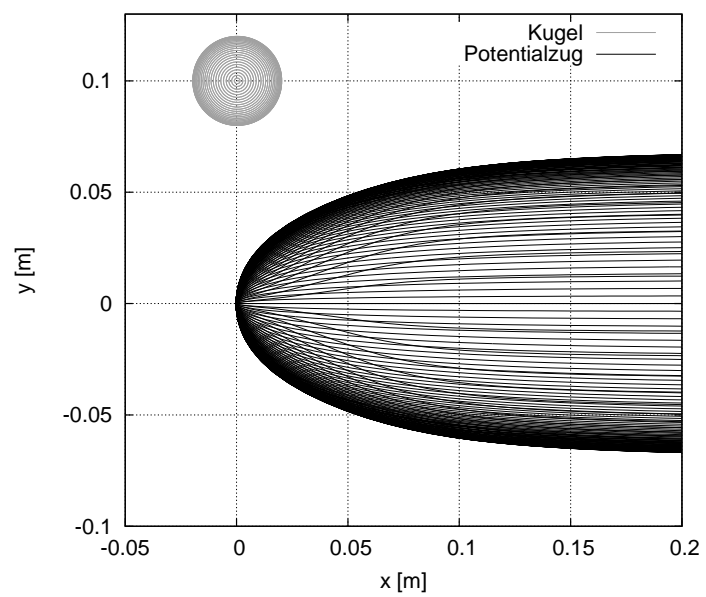

Abbildung 2.8.: Blick von oben auf den Potentialzug und die Kugel $R=2 \mathrm{~cm}$ bei $y_{M}=$ $10 \mathrm{~cm}$ an der Position $x=0$.

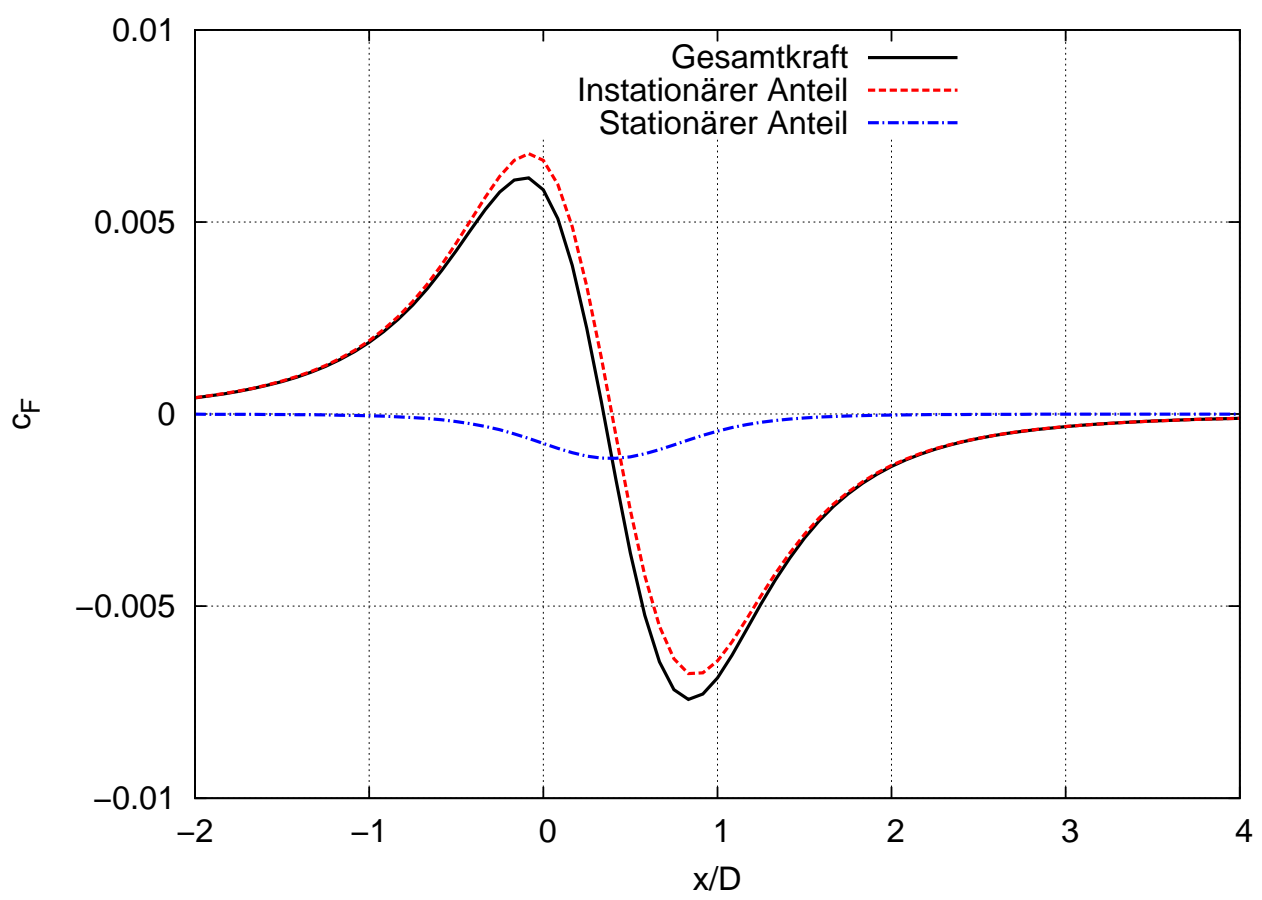

Abbildung 2.9.: Getrennte Berechnung des Kraftbeiwertes aus dem stationären und dem instationärem Anteil der Bernoulli-Gleichung (schwarz: Gesamtkraft, rot: instationärer Anteil, blau: stationärer Anteil). 


\subsubsection{Berechnung der Kraft auf eine Kugel mit akustischem Ansatz}

Die Ergebnisse aus dem vorigen Abschnitt haben gezeigt, dass der instationäre Anteil des Strömungspotentials $\partial \phi / \partial t$ der entscheidende Anteil der Kraft auf die Kugel in der instationären Strömung ist. Das Druckfeld eines fahrenden Zuges ist eigentlich dreidimensional (siehe Abbildung 2.3.2(a)). Ist die Kugel aber klein gegenüber dem Zug, kann die Druckwelle, die auf die Kugel trifft, näherungsweise als ebene Welle betrachtet werden (siehe Abbildung 2.3.2(b)).

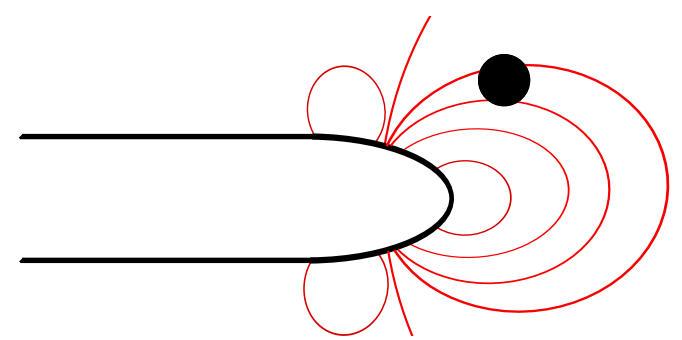

(a) 3D-Druckfeld um den Zug.

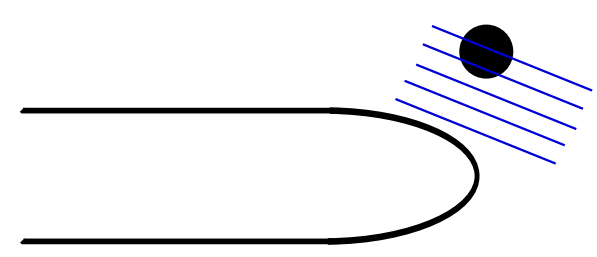

(b) Vereinfachtes 2D-Druckfeld um den Zug.

Abbildung 2.10.: Kugel im Druckfeld eines fahrenden Zuges (schematische Darstellung).

Die Situation erscheint ähnlich dem Fall, wenn eine ebene akustische Welle an einer Kugel gestreut wird [25]. Dabei wird das Geschwindigkeitsfeld in zwei Anteile zerlegt: in das Feld der einfallenden Welle $\left(\vec{u}_{\text {ein }}\right)$ und in das Feld der gestreuten Welle $\left(\vec{u}_{\text {ges }}\right)$. Auf der Kugeloberfläche gilt

$$
\left(\vec{u}_{\text {ein }}+\vec{u}_{\text {ges }}\right) \cdot \vec{n}=0,
$$

mit $\vec{n}$ als Normalenvektor der Kugeloberfläche.

Der Druck der einfallenden Welle kann folgendermaßen ausgedrückt werden [25]:

$$
p_{\text {ein }}=A \cos \left(\omega t-k x_{1}\right) .
$$

Die Taylor-Entwicklung dieser Druckwelle lautet folgendermaßen

$$
p_{\text {ein }}=p_{0}+\frac{\partial p}{\partial x_{1}} \Delta x_{1} .
$$

Die Druckwelle wird bis zum ersten Taylor-Glied angenähert, das ist erlaubt, wenn der Radius der Kugel sehr viel kleiner als die Wellenlänge der einfallenden Welle ist, was hier der Fall ist. Mit $\Delta x_{1}=R \cdot \cos \theta$ mit $R$ dem Radius der Kugel, eingesetzt in 2.33 gilt dann für $p_{\text {ein }}$ auf der Oberfläche der Kugel:

$$
p_{\text {ein }}=\frac{\partial p}{\partial x_{1}} \cdot R \cdot \cos \theta .
$$




\section{Analytische Berechnung der Zugvorbeifahrt mittels Potentialtheorie}

Für die Geschwindigkeit kann folgender Ausdruck verwendet werden [25]:

$$
\vec{u}_{\text {ein }}=\mathfrak{R e}\left\{\hat{u_{k}} e^{i \omega t}\right\},
$$

mit der Amplitude $\hat{u}_{k}$. Die zeitliche Ableitung lautet dann entsprechend:

$$
\frac{\partial \vec{u}}{\partial t}=i \omega \hat{u}_{k} e^{i \omega t}
$$

mit $\frac{\partial p}{\partial x_{1}}=\rho_{0} \cdot \frac{\partial u}{\partial t}$ gilt dann für die einfallende Welle:

$$
p_{\text {ein }}=\hat{u}_{k} i \omega e^{i \omega t} \rho_{0} R \cos \theta \text {. }
$$

Nun wird die gestreute Welle an der oszillierenden Kugel berechnet. Der Druck der gestreuten Welle lautet:

$$
p_{\text {ges }}=\hat{p}(\vec{x}) \cdot e^{i \omega t} .
$$

mit $\hat{p}=\hat{u}_{R} i \omega \frac{\rho_{0} R}{2}$ gilt auf der Kugeloberfläche [25]:

$$
p_{\text {ges }}=\hat{u}_{R} i \omega \frac{\rho_{0} R}{2} e^{i \omega t} .
$$

Mit der Randbedingung $\hat{u}_{R}=\hat{u}_{k} \cdot \cos \theta$ folgt für die gestreute Welle:

$$
p_{\text {ges }}=\frac{1}{2} \hat{u}_{k} \cdot i \omega \rho_{0} R \cdot \cos \theta e^{i \omega t}=p_{\text {ein }} / 2 \text {. }
$$

Der Gesamtdruck $p_{\text {tot }}$ lautet dann also:

$$
p_{\text {tot }}=p_{\text {ein }}+p_{\text {ges }}=3 \cdot p_{\text {ges }} .
$$

Die Kraft ergibt sich aus der Integration des Drucks über die Kugeloberfläche:

$$
\begin{aligned}
F & =\int_{S} p_{\text {tot }} \cdot \cos \theta d S \\
F & =\int_{S} 3 \cdot p_{\text {ges }} \cdot \cos \theta d S \\
F & =\int_{S} \hat{u}_{k} i \omega \frac{3}{2} \rho_{0} R e^{i \omega t} \cos ^{2} \theta d S \\
F & =\hat{u}_{k} \cdot i \omega \frac{3}{2} \rho_{0} R e^{i \omega t} \int_{S} \cos ^{2} \theta d S
\end{aligned}
$$

mit

$$
\int_{S} \cos ^{2} \theta d S=\frac{4}{3} \pi R^{2}
$$

und Gleichung 2.36 eingesetzt in 2.45 folgt der folgende Ausdruck für die Kraft auf die Kugel:

$$
F=2 \pi R^{3} \frac{\partial u}{\partial t} \rho_{0}=2 \pi R^{3} \nabla p
$$


Der Druckgradient $\nabla p$ wird um den Mittelpunkt der Kugel mit einem Differenzenquotienten berechnet, hier in $x$-Richtung, entsprechend in $y$ - und $z$-Richtung

$$
\frac{\partial p}{\partial x}=\frac{p\left(x_{M}-d x\right)-p\left(x_{M}\right)}{d x} .
$$

Abbildung 2.11 zeigt den Kraftbeiwert $c_{F}$ in $x$ - und $y$-Richtung auf eine Kugel bei der Vorbeifahrt, berechnet mit der Integration über die Kugeloberfläche, beschrieben in Abschnitt 2.3, und berechnet mit dem akustischen Modell, welches in diesem Abschnitt hergeleitet wurde. Bei $D$ handelt es sich um eine Standard-Zugbreite von $12 \mathrm{~cm}$. Beide Modelle liefern fast identische Ergebnisse, lediglich am Maximum beträgt die maximale Abweichung zwischen beiden Modellen 10\%. Da die Berechnung mit der Methode der oszillierenden Kugel viel weniger rechenintensiv ist (wenige Sekunden Rechenzeit gegenüber mehreren Minuten auf einem PC) als die Berechnung und Integration des Druckes über die gesamte Kugeloberfläche bei jedem einzelnen Zeitschritt, wird für die zukünftigen Berechnungen der Kraft auf Objekte bei der Vorbeifahrt dieses vereinfachte Modell verwendet. Ist die Kugel klein gegenüber dem Zugkopf, was hier der Fall ist, ist die Näherung hinreichend genau. Die Berechnung der Dipolmomente entfällt, es muss lediglich das Strömungsfeld des Potentialzugs berechnet werden und der Druckgradient im Mittelpunkt einer gedachten Kugel, um die Kraft zu erhalten. Auch dieser Punkt wird quasistatisch am Zug vorbei bewegt, um einen Kraftverlauf über die Position zu erhalten.

\subsubsection{Kräfte auf ebene Platten}

Nicht nur Personen am Bahnsteig sind großen Kräften bei der Zugvorbeifahrt ausgesetzt, auch Schilder und Signalanlagen auf offener Strecke müssen den starken Drucklasten durch die Kopfwelle standhalten können, da der Zug auf offener Strecke meist eine viel höhere Geschwindigkeit hat als bei der Durchfahrt durch einen Bahnhof. Im folgenden Abschnitt werden die Kräfte auf kreisförmige und rechteckige Platten bei der Zugvorbeifahrt analytisch berechnet.

\section{Kraft auf eine Kreisscheibe}

Das Modell der Streuung einer akustischen Welle an einer Kugel funktioniert auch mit anderen Objekten, zum Beispiel einer Kreisscheibe. Lord Rayleigh hat dieses Problem theoretisch untersucht [26]. Sei $\phi$ das Geschwindigkeitspotential der oszillierenden Scheibe. Die kinetische Energie an einer Seite beträgt $E_{k i n}=h u^{2}$, wobei es sich bei $h$ um einen objektspezifischen Wert handelt. Nach dem Green'schen Theorem folgt daraus:

$$
\begin{aligned}
h u^{2} & =-\iint \phi \frac{d \phi}{d n} d S=-u \iint \phi d S \\
& \Rightarrow-h u=\iint \phi d S \\
& \Rightarrow-h \dot{u}=\iint \frac{\partial \phi}{\partial t} d S
\end{aligned}
$$




\section{Analytische Berechnung der Zugvorbeifahrt mittels Potentialtheorie}

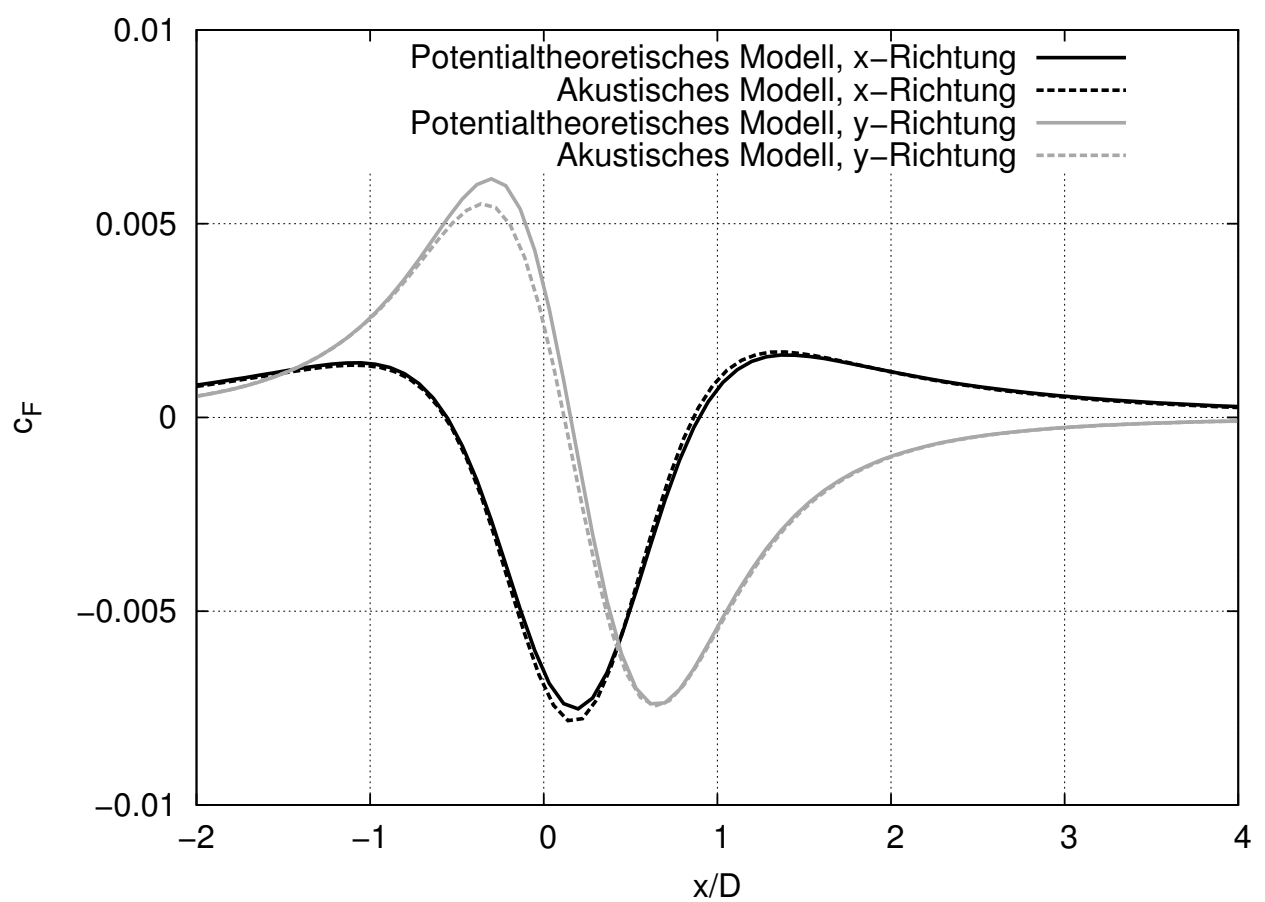

Abbildung 2.11.: Kraft auf die Kugel durch die Kopfwelle, Vergleich der beiden theoretischen Modelle.

Mit $\frac{\partial \phi}{\partial t}=-p / \rho_{0}$ folgt:

$$
\begin{aligned}
& \Rightarrow h \dot{u}=\iint \frac{p}{\rho_{0}} d S \\
& \Rightarrow h \dot{u}=F / 2 \\
& \Rightarrow \quad F=2 h \dot{u} \rho_{0}
\end{aligned}
$$

Für eine Kreisschreibe mit Radius $R$ gilt $h=\frac{4 R^{3}}{3}$ [27]. So ergibt sich für die Kraft auf die Kreisscheibe:

$$
F=\frac{8}{3} R^{3} \frac{\partial u}{\partial t} \rho_{0}=\frac{8}{3} R^{3} \nabla p
$$

Die Kraft auf die Scheibe ist also um den Faktor 2,3 kleiner als die Kraft auf eine Kugel mit demselben Radius. Für eine dünne Scheibe mit der Dicke $d$ wird der Ausdruck wie folgt erweitert [28]:

$$
F=\frac{8}{3} \frac{\partial u}{\partial t} \rho_{0} R^{3}\left[1-(4-\pi) \frac{d}{8 R}\right]
$$

\section{Kraft auf eine rechteckige Platte}

In [29] werden Kräfte auf rechteckige Platten potentialtheoretisch berechnet und mit experimentellen Daten verglichen. Auch hierbei werden die Kräfte auf das Objekt durch 
die Kopfwelle eines Potentialzugs induziert. Allerdings verwenden die Autoren für die Zuggeometrie nur eine Punktquelle. Eine weitere Einschränkung wird beim Objekt gemacht: Es wird vorausgesetzt, dass die Platte auf dem Boden steht und viel höher ist als breit, so dass die Strömung um die Platte zweidimensional berechnet werden kann. Zudem wird die Dicke $d$ als unendlich dünn angenommen.

Die Kraft auf eine rechteckige Platte lautet dann wie folgt [29]:

$$
F=c_{F} \cdot \frac{1}{2} \rho u_{\infty}^{2} \cdot 2 B
$$

mit $2 \cdot B$ als Breite. Je nach Orientierung der Platte zur Strecke gibt es verschiedene Kraftbeiwerte. Ist die Platte parallel zur Strecke gilt [29]:

$$
c_{F P}=-\frac{3}{4} c_{s} \frac{T}{\left[T^{2}+1\right]^{5 / 2}},
$$

mit der dimensionslosen Zeit

$$
T=\frac{t \cdot u_{\infty}}{y_{M}}=\frac{x}{y_{M}} .
$$

Normal zur Strecke gilt [29]:

$$
c_{F N}=\frac{1}{4} c_{s} \frac{2 T^{2}-1}{\left[T^{2}+1\right]^{5 / 2}} .
$$

Mit

$$
c_{s}=\frac{B \cdot A}{y_{M}^{3}}
$$

mit $A$ als der Querschnittfläche des Zuges. Bei der Orientierung der Platte normal zur Fahrtrichtung, also für $c_{F N}$ ist zu beachten, dass statt $y_{M}=-y_{M}$ eingesetzt wird.

\subsection{Vergleich der aerodynamischen Lasten durch verschiedene Zuggeometrien}

Zu Beginn dieses Kapitels wurde erwähnt, dass Züge, um für den europäischen Schienenverkehr zugelassen zu werden, gewisse Anforderungen erfüllen müssen, die in der TSI-Norm definiert sind [13]. Für die Sicherheit von Personen am Bahnsteig sind nur die Geschwindigkeiten am Gleis an verschiedenen Positionen gefordert. Beispielsweise darf ein durchfahrender Zug an der Position $y_{M}^{\prime}=3 \mathrm{~m}$ bei einer Geschwindigkeit von $u_{\infty}=55,55 \mathrm{~m} / \mathrm{s}$ den Wert $u_{\max }=15,5 \mathrm{~m} / \mathrm{s}$, gemessen in der $x$ - $y$-Ebene und $240 \mathrm{~mm}$ über der Schienenoberkante, nicht überschreiten [13]. Dieser Wert gilt für alle Züge unabhängig von ihrer Geometrie. Übersteigt ein Zug diesen Wert, wird er für den europäischen Bahnverkehr nicht zugelassen. 


\section{Analytische Berechnung der Zugvorbeifahrt mittels Potentialtheorie}

Abbildung 2.12 zeigt den Betrag der Geschwindigkeiten $|u|=\sqrt{u^{2}+v^{2}+w^{2}}$ am TSI-Messpunkt im 1:25 Modell, ausgelöst durch die drei verschiedenen Zuggeometrien. Der in Abschnitt 2.2.1 vorgestellte Dreiquellenzug, ein Punktquellenzug mit der Quelle im Koordinatenursprung und der in Abschnitt 2.2.2 vorgestellten Kastenzug. Der Verdrängungsquerschnitt beträgt bei allen drei Zügen $A=0,016 \mathrm{~m}^{2}$, die Anströmgeschwindigkeit beträgt $u_{\infty}=40 \mathrm{~m} / \mathrm{s}$, der Abstand zur Gleismitte ist $y_{M}=$ $0,12 \mathrm{~m}$. Es ist deutlich zu erkennen, dass der kastenförmige Zug sogar einen niedrigeren Geschwindigkeitsbetrag am Gleis erreicht als die beiden schlankeren Geometrien.

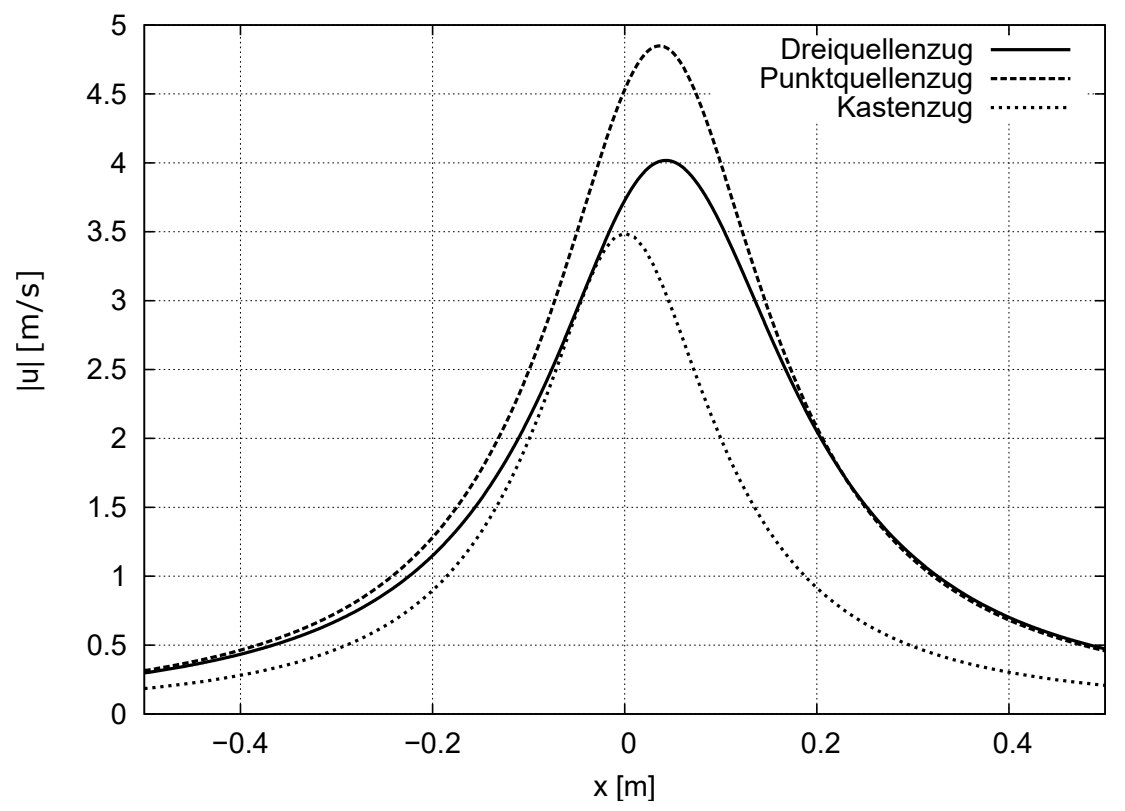

Abbildung 2.12.: Betrag der Geschwindigkeiten am Ort der Kugel, induziert durch drei verschiedene Zugkkopfgeometrien: Punktquelle, Dreifachquelle, Kastenzug $\left(y_{M}=0,12 \mathrm{~m}\right.$ und $\left.u_{\infty}=40 \mathrm{~m} / \mathrm{s}\right)$.

Um nun zu zeigen, welchen Einfluss die Zuggeometrie auf die Kraft auf eine Kugel hat, sind in Abbildung 2.13 die aerodynamischen Lasten auf eine Kugel mit einem Radius von $R=2 \mathrm{~cm}$ ausgelöst durch die drei verschiedenen Zugkopfgeometrien dargestellt. Es ist deutlich zu erkennen, dass unterschiedliche Zuggeometrien unterschiedliche Lasten auf die Kugel ausüben. Der Dreiquellenzug hat, aufgrund der Linienquellverteilung in der $x-z$-Ebene, den schlankesten Zugkopf. Der Punktquellenzug hat, da er nur eine Quelle im Zugkopf hat und die dadurch entstehende Kontur eine etwas dickere Ellipse ist, einen stumpferen Kopf als der Dreiquellenzug. Der Kastenzug hat eine sehr stumpfe Kopfform.

Abbildung 2.13 ist zu entnehmen, dass der Punktquellenzug bereits eine circa 25\% höhere Last auf die Kugel ausübt als der Dreiquellenzug. Der Kastenzug übt sogar eine $300 \%$ höhere Last auf die Kugel aus als der Dreiquellenzug. 


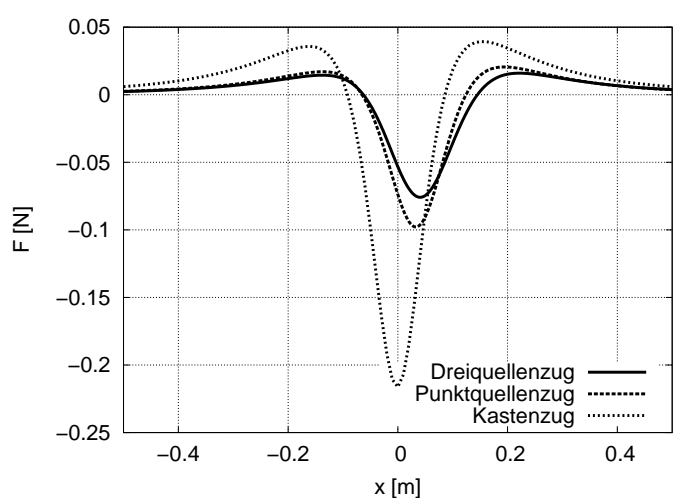

(a) x-Richtung

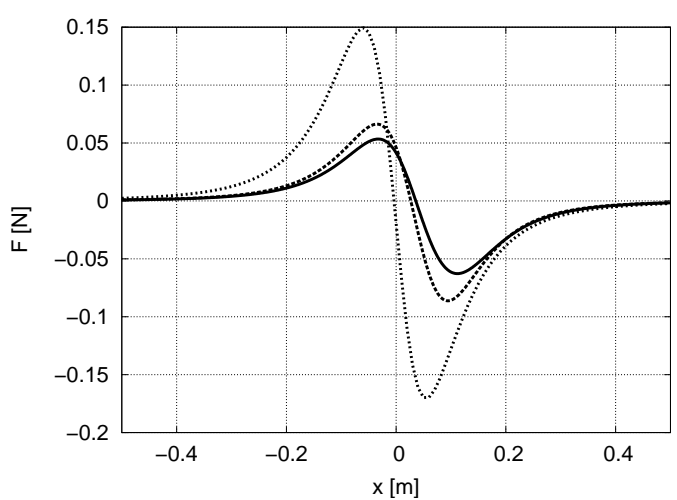

(b) y-Richtung

Abbildung 2.13.: Vergleich der Kräfte auf eine Kugel $R=2 \mathrm{~cm}$, bei $y_{M}=12 \mathrm{~cm}$ und $u_{\infty}=40 \mathrm{~m} / \mathrm{s}$ induziert durch verschiedene potentialtheoretischer Zuggeometrien: Punktquelle, Mehrfachquelle, Kastenzug.

Allein diese theoretischen Vergleiche zeigen, dass unterschiedliche Zugkopfgeometrien stark unterschiedliche Lasten auf Objekte ausüben.

Wenn Zuggeometrien allein durch die Geschwindigkeit am Gleis die in der Norm geforderten Grenzwerte erfüllen, kann die Kraft ausgelöst durch die Kopfwelle trotzdem viel höher und dadurch gefährlicher sein. Diese Untersuchung zeigt, wie wichtig es ist, die aus der Wirkung der Kopfwelle entstehenden Kräfte bestimmen zu können. Die Betrachtung der maximalen Geschwindigkeit am Gleis ist alleine unzureichend zur Beurteilung des Gefahrenpotentials. 

KAPITEL 3

Die Tunnel-Simulations-Anlage Göttingen (TSG)

\subsection{Aufbau der Versuchsanlage}

In diesem und in den folgenden Kapiteln werden die Experimente beschrieben, die in der Tunnel-Simulations-Anlage Göttingen (TSG) durchgeführt werden. Die Versuchsanlage verwendet bewegte Modelle (engl. „moving model rig“). Dies bedeutet, dass im Gegensatz zu einem konventionellen Windkanal, in dem ein unbewegtes Modell angeströmt wird, die aerodynamischen Untersuchungen an einem bewegten Modell durchgeführt werden. Der entscheidende Vorteil ist, dass transiente Vorbeifahrten des Modells an Objekten realitätsnah untersucht werden können. Dies lässt sich in konventionellen Windkanälen nicht durchführen. Die Anlage ist weltweit in dieser Konfiguration einzigartig. Es gibt nur wenige vergleichbare Anlagen, wie zum Beispiel das „train rig“ an der Universität Birmingham [30].

Die in der TSG durchgeführten Experimente dienen dem Vergleich mit denen in Kapitel 2 vorgestellten theoretischen Modellen und der Untersuchung etwaiger Abweichungen.

Die TSG besteht aus vier Bereichen: dem Katapult, dem Zwischenbereich, dem Plenum und der Bremse (siehe Abbildung 3.1). Die Strecke hat eine Länge von 60 Metern (siehe Abb. 3.1). Die gesamte Anlage ist von einer Einhausung umgeben, so dass zum Einen im Betrieb keine Personen in den Gefahrenbereich gelangen können und zum Anderen, sollte es zu einem Unfall kommen, keine Modelle oder Teile der Anlage nach außen gelangen können. Die Modelle werden mithilfe eines hydraulischen Katapults beschleunigt, und fahren anschließend frei durch die Messstrecke.

Das Katapult zur Beschleunigung des Zugmodells ähnelt im Grundaufbau einer Armbrust. Allerdings gibt es bedeutende Unterschiede. Die benötigte Energie zur Beschleunigung des Modells wird nicht in einer mechanischen Feder gespeichert und direkt von dem Seil auf das Modell übertragen, wie es bei einer Armbrust mit einem Pfeil der Fall ist. 


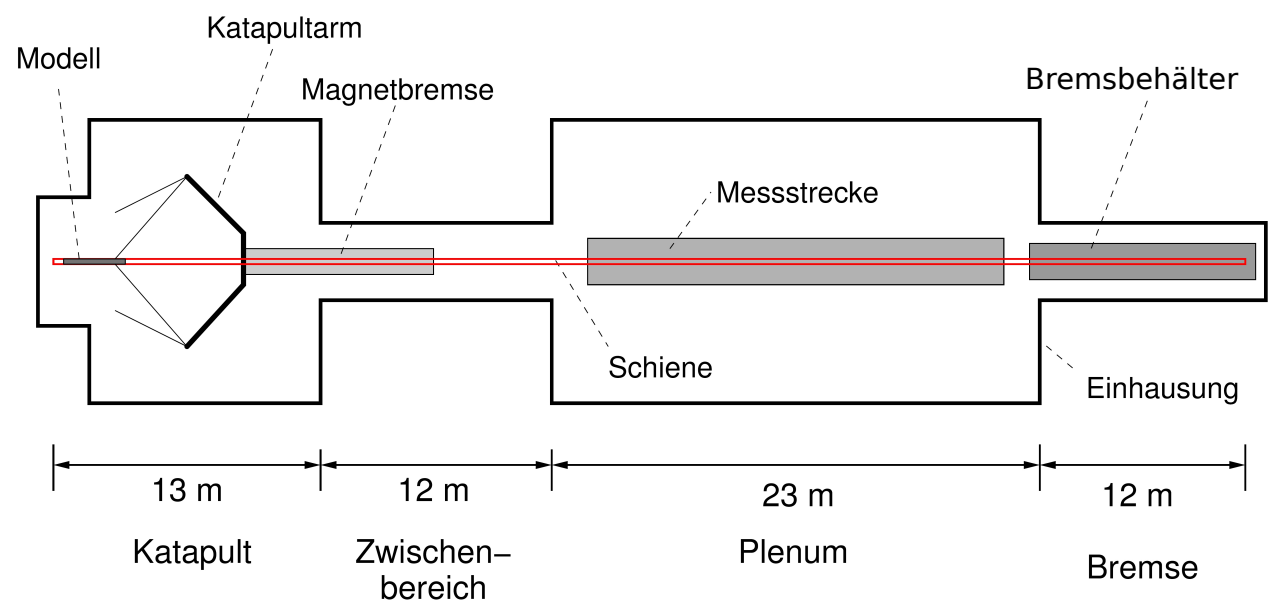

Abbildung 3.1.: Schematische Darstellung der TSG [2].

Die zwei Meter langen Hebelarme des Katapults werden stattdessen hydraulisch angetrieben, wobei beide Arme nicht mechanisch gekoppelt sind. An beiden Armen befindet sich je ein hydraulischer Zylinder, welche zueinander achsensymmetrisch zur Laufstrecke des Modells angeordnet sind (siehe Abb. 3.2).

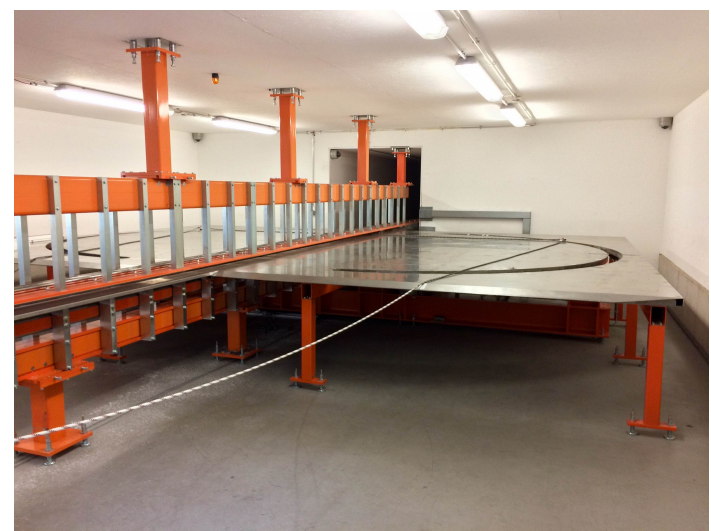

(a) Foto vom Katapultbereich.

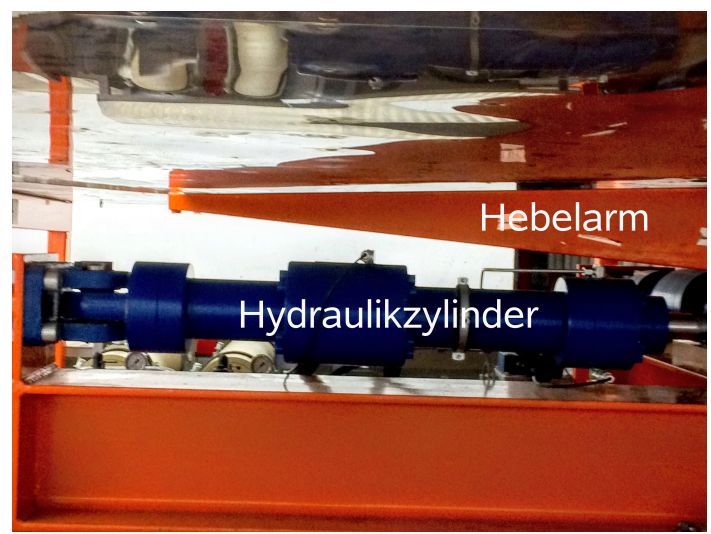

(b) Hebelarm und Hydraulikzylinder auf der linken Seite in Fahrtrichtung unter dem Tisch.

Abbildung 3.2.: Katapult zur Beschleunigung der Modelle in der Versuchsanlage.

Zum Beschleunigen der Modelle wird ein hoher Druck benötigt. Dazu wird ein so genannter Vordruck eingestellt. Der Druck, welcher letztendlich die Geschwindigkeit der Modelle bestimmt, wird Arbeitsdruck genannt. Dieser hängt wiederum vom Modellgewicht ab. Will man bei schwereren Modellen dieselbe Geschwindigkeit erreichen wie bei 
leichteren Modellen, muss entsprechend ein höherer Druck eingestellt werden.

Beim Abschuss des Modells öffnet sich bei beiden Antriebszylindern je ein Ventil, wodurch schlagartig ein hoher Druck auf die Zylinder gegeben wird. Da beide Ventile dasselbe Auslösesignal erhalten, geschieht dieses auf beiden Seiten annähernd zeitsynchron. Die Kraft der Zylinder bewirkt ein starkes Drehmoment in den Hebelarmen, wodurch sich diese in Bewegung setzen. Ein weiterer Unterschied zu einer Armbrust ist, dass die Hebelarme über ein Seil nicht das Modell direkt beschleunigen, sondern stattdessen einen Hilfsschlitten, an welchen das Modell angelehnt wird. Dies hat zum einen den Vorteil, dass das Modell keine seitlichen Zugkräfte durch eventuell leicht asynchrones Anziehen der Hebelarme erfährt. Zum Anderen sind, bis auf das Anbringen eines kleinen Hakens am Unterboden des Modells zum Anlehnen an den Hilfsschlitten, keine weiteren Modifikationen der Geometrie notwendig. Eine schematische Darstellung der Kraftübertragung auf das Modell durch den Hilfsschlitten befindet sich in Abbildung 3.3.

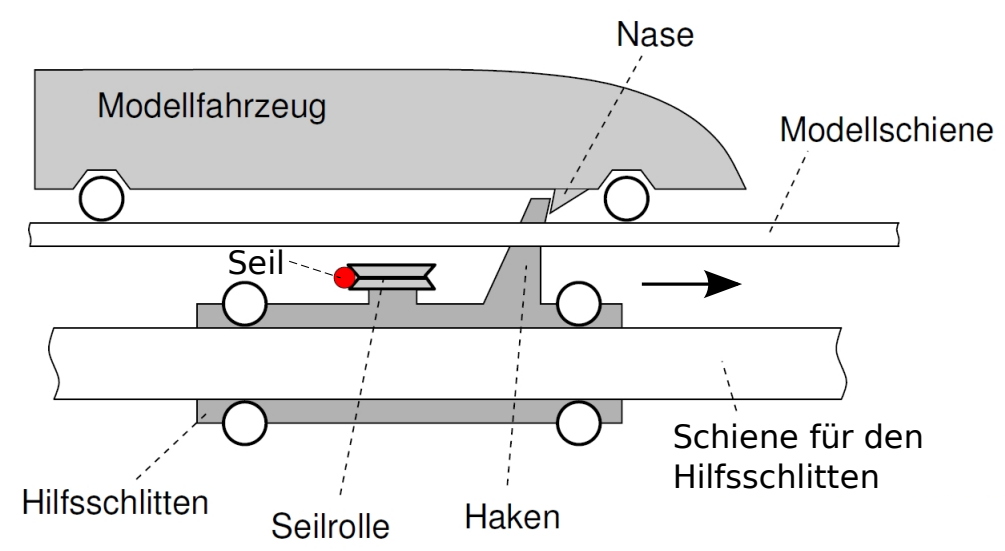

Abbildung 3.3.: Schematische Darstellung der Kraftübertragung auf das Modell durch einen Hilfsschlitten [2].

Zwischen den Drehpunkten der Hebelarme befindet sich eine Wirbelstrombremse, die den Schlitten abbremst. Das Modell fährt fortan frei mit geradlinig gleichförmiger Bewegung durch den Zwischenbereich in die Messstrecke hinein. In dem Zwischenbereich können eventuell vorhandene Schwingungen des Modells, die bei der Beschleunigung durch das Katapult angeregt wurden, abklingen. Die Modelle verfügen über kleine Sicherheitshaken, welche das Modell im Notfall am Verlassen der Schienen hindern.

Die Messstrecke ist 23 Meter lang. Die Strecke kann mit verschieden Böden, zum Beispiel einen Bahndamm oder einem flachen Boden ausgestattet werden (siehe Abbildung 3.4). Es können aber auch Tunnel, durch die der Zug hindurch fährt oder Objekte aufgestellt werden, an denen der Zug vorbeifährt. Zudem existiert ein Windkanal zur Seitenwinderzeugung. Es können also auch plötzliche Lasten auf das Modell, ausgelöst durch Seitenwind, untersucht werden. In der Messstrecke befinden sich Lichtschranken, welche die 


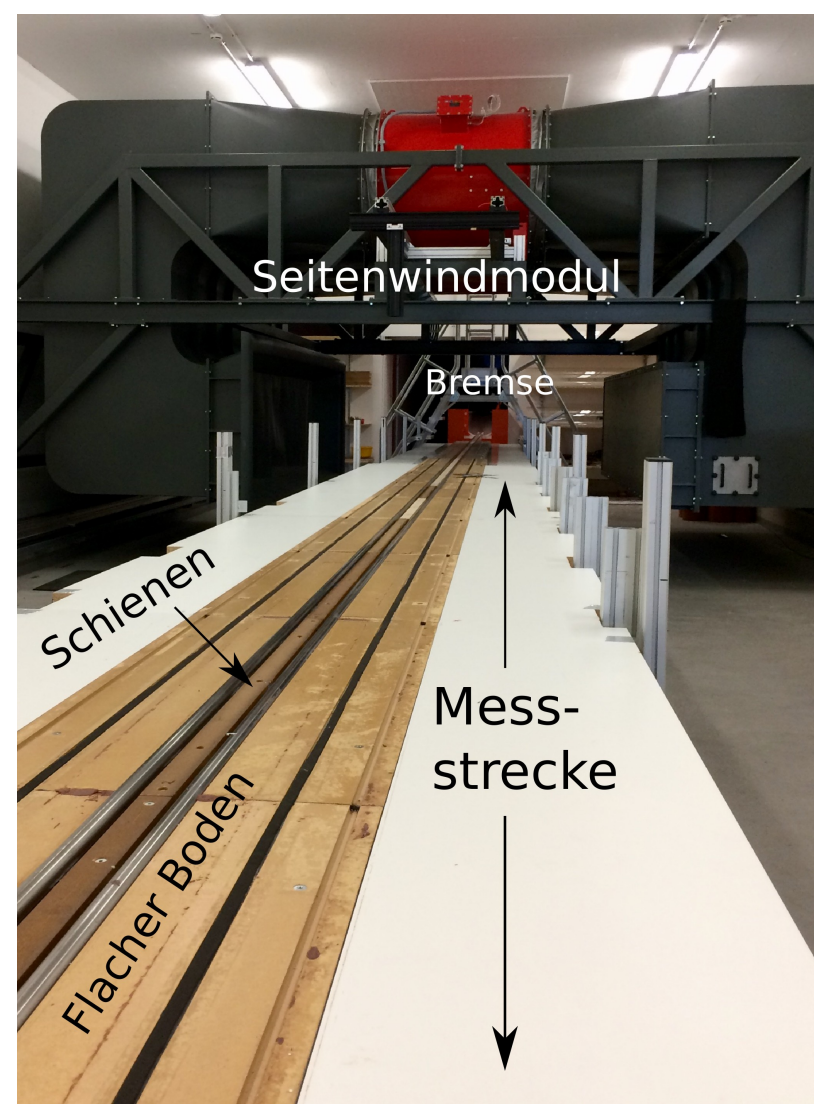

Abbildung 3.4.: Messstrecke mit Seitenwindmodul.

Geschwindigkeit des durchfahrenden Zuges messen. Weiterhin dienen diese zur Kontrolle, ob der Zug auf seinem Weg durch die Messstrecke, aufgrund von Reibung und Luftwiderstand, stark an Geschwindigkeit verliert. In der Messstrecke befinden sich im Abstand von 1,245 Metern zwei Lichtschranken. Diese messen jeweils die Geschwindigkeit des Zuges an ihrem jeweiligen Ort. Um zu überprüfen, wie hoch die negative Beschleunigung des Zuges aufgrund von Reibung und Luftwiderstand ist, wurden bei allen Messungen die Werte der Lichtschranken mit aufgenommen. Damit wurde die mittlere Verzögerung $a$ aus 20 Versuchen mit dem Potentialmodell bestimmt. Der Wert $\sigma_{v}$ gibt den mittleren prozentualen Unterschied der gemessenen Geschwindigkeiten zwischen der zweiten und der ersten Lichtschranke an.

$$
a=(-0,7 \pm 0,2) \frac{\mathrm{m}}{\mathrm{s}^{2}}, \quad \sigma_{v}=(-2,1 \pm 0,5) \% .
$$

Der Geschwindigkeitsverlust ist also nur sehr gering, es kann von nahezu geradlinig gleichförmiger Geschwindigkeit ausgegangen werden. Des weiteren zeigt diese Untersuchung, dass die Geschwindigkeiten in der TSG sehr reproduzierbar einstellbar sind. 
Nach dem Durchfahren der Messstrecke fährt der Zug in einen zwölf Meter langen Bereich, in dem um die Strecke herum eine Box mit Deckel angebracht ist, welche mit Schüttgut bestehend aus kleinen Polystyrolkügelchen (Durchmesser 1-3 mm) befüllt ist. Dort soll der Zug möglichst sanft abgebremst werden. Diese Box wird im Folgenden als Bremsbehälter bezeichnet. In Abbildung 3.5 ist der Bremsbehälter einmal bei geöffnetem Deckel zu sehen (Abb. 3.5(a)) und einmal von vorne bei geschlossenem Deckel (Abb. 3.5(b)). Auf den Aufnahmen ist zu sehen, dass zusätzlich ein Zwischenblech angebracht ist. Versuche haben gezeigt, dass ohne Zwischenbleche eine starke Auftriebskraft am Modell entstehen kann [2]. Durch das Zwischenblech wird bei der Einfahrt des Modells eine direkte Verdrängung des Schüttguts nach oben behindert, so dass es hauptsächlich zur Seite ausweicht. Zusätzlich kann das Zwischenblech in der Höhe variiert werden. Gemeinsam mit der Höhe der Schüttung beeinflussen diese beiden Parameter die Stärke der Bremswirkung. Durch Anpassung der beiden Höhen kann die Bremswirkung optimiert werden, um eine möglichst gleichmäßige Verzögerung des Modells zu erreichen und auch für verschieden Modelle zu variieren. Im Gegensatz zur Beschleunigung, bei der die Krafteinleitung über den Haken am Hilfsschlitten an einer definierten Position im Unterbodenbereich stattfindet, greift im Bremsbehälter die Kraft auf der gesamten Oberfläche des Modells an. Dies muss bei der Konstruktion berücksichtigt werden und erfordert speziell im Bereich des Zugkopfes eine entsprechende Verstärkung. Etwaige Anbauteile sollten ebenfalls entsprechend robust ausgelegt werden.

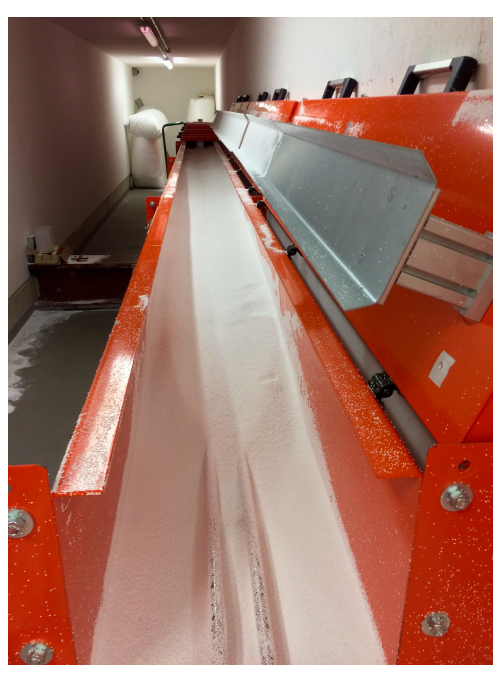

(a) Bremse mit geöffnetem Deckel, Ansicht von oben.

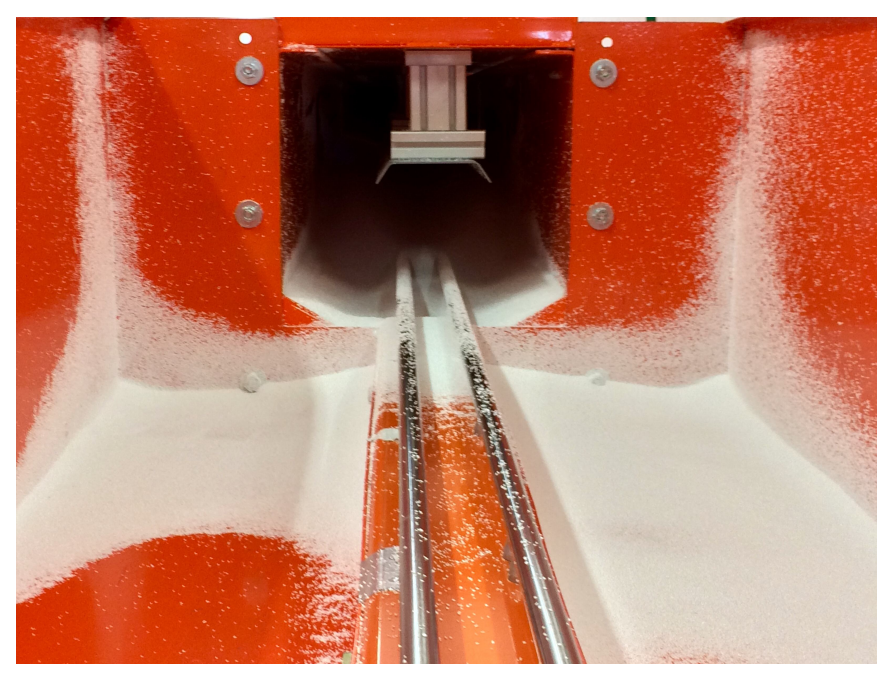

(b) Bremse mit geschlossenem Deckel, Ansicht von vorn.

Abbildung 3.5.: Ansicht der Bremsbox der TSG. 


\section{Die Tunnel-Simulations-Anlage Göttingen (TSG)}

\subsection{Verwendete Modellzüge}

Die Anlage ist für Modelle im Maßstab 1:25 mit einem Gewicht von bis zu $10 \mathrm{~kg}$ ausgelegt. Je schwerer ein Modell ist, desto geringer ist die mit dem Katapult erreichbare Endgeschwindigkeit. Da die Modelle hohen Beschleunigungen im Katapult- und Bremsbereich standhalten müssen, werden sie meist aus Kohlefaserverbundwerkstoffen gefertigt. Für die Experimente in der TSG werden folgende Modellzüge im Maßstab 1:25 verwendet: der Potentialzug, welcher nach den Geometriedaten des berechneten Dreiquellenzuges aus Kapitel 2.2.1 konstruiert wurde, ein dreiteiliges ICE3 Modell, wobei Triebwagen und Heck identisch sind und ein NGT-Modell. NGT steht für „Next Genereation Train“ [31]. Dabei handelt es sich um eine Konzeptstudie des Deutschen Zentrums für Luft- und Raumfahrt (DLR). Der NGT ist ein Doppelstockzug, welcher ohne Stromabnehmer auskommen soll, da die Energie induktiv über die Schiene übertragen werden soll. Zudem soll er in Ultraleichtbauweise konstruiert werden und nur halb so viel Energie pro Passagier verbrauchen, wie aktuelle Hochgeschwindigkeitszüge [31]. Die Geometriedaten der Modelle sind Tabelle 3.1 zu entnehmen und in Abbildung 3.6 sind die einzelnen Zugmodelle neben dem Kraftmesselement zu sehen.

\begin{tabular}{l|c|c|c} 
& ICE3 & Potentialzug & NGT \\
\hline Länge $[\mathrm{m}]$ & 2,75 & 2,26 & 2,36 \\
Höhe $[\mathrm{m}]$ & 0,145 & 0,144 & 0,176 \\
Querschnittfläche $\left[\mathrm{m}^{2}\right]$ & 0,0155 & 0,0160 & 0,0193 \\
Nasenlänge $[\mathrm{m}]$ & 0,26 & 0,15 & 0,35
\end{tabular}

Tabelle 3.1.: Vergleich der Geometrien der verwendeten Modellzüge.

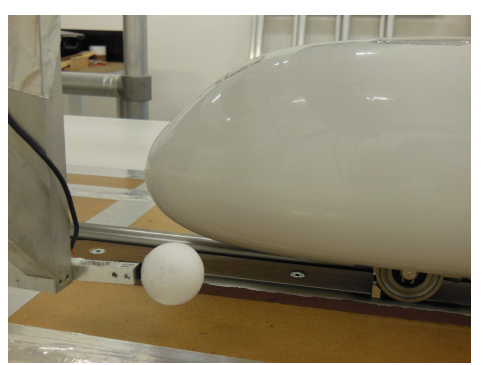

(a) Potentialzug

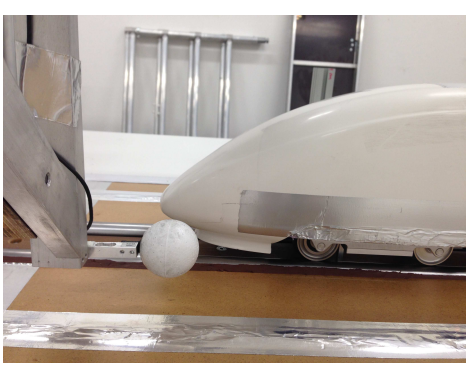

(b) ICE3

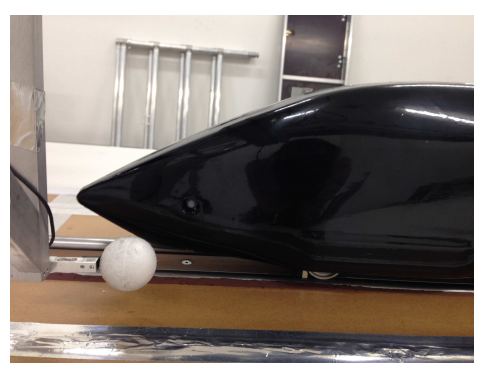

(c) NGT

Abbildung 3.6.: Für die Vorbeifahrtmessungen verwendete Zugmodelle: a) Potentialzug, b) ICE3 und c) Next Generation Train (NGT). 
KAPITEL 4

Geschwindigkeitsmessungen an der TSG mithilfe von Hitzdrahtanemometrie

Zur Überprüfung, ob die Potentialtheorie im Experiment anwendbar ist, werden Geschwindigkeitsmessungen des Strömungsfeldes an bestimmten Punkten neben dem Zugmodell in der TSG vorgenommen. Diese dienen dazu, zu überprüfen, ob das Strömungsfeld, welches vom Zugmodell erzeugt wird, dem theoretisch berechneten Strömungsfeld um den Zug entspricht.

Die Geschwindigkeitsmessungen können Aufschluss darüber geben, ob das konstruierte Potentialzugmodell nicht mehr dem berechneten Dreiquellenmodell entspricht. Gründe hierfür könnten sein, dass das real konstruierte Modell Räder hat, und entsprechend zwei Achsen, was in der Theorie nicht der Fall ist. Daher können Turbulenzen auftreten, welche von der Potentialtheorie nicht erfasst werden können.

\subsection{Verwendete Messtechnik}

Die Geschwindigkeitsmessungen werden mithilfe von Hitzdrahtanemometrie durchgeführt. Dafür wird das Streamline 90N10 Messsystem der Firma Dantec verwendet [32]. Die Kalibrierung erfolgt mit der Software StreamWare Version 1.16 ebenfalls von der Firma Dantec.

Die Entscheidung für das Messsystem fiel auf die Hitzdrahtanemometrie, da sie die Messung der lokalen Geschwindigkeit und die Auflösung sehr kleinskaliger Fluktuationsgeschwindigkeiten ermöglicht [33]. Die Geschwindigkeit kann zeitlich sehr hochaufösend gemessen werden, typischerweise bis in den Bereich von 5 bis $10 \mathrm{kHz}$. Diese Messmethode ist recht kostengünstig und es sind keine besonderen Schutzmaßnahmen erforderlich. Allerdings sind die Hitzdrahtsonden sehr empfindlich gegen Verschmutzung und werden schnell funktionsuntüchtig. 


\section{Geschwindigkeitsmessungen an der TSG mithilfe von Hitzdrahtanemometrie}

Alternative Messverfahren wären beispielsweise Drucksonden wie Prandtl-Sonden, diese haben aber den Nachteil, dass sie bei Messungen in Luft unterhalb von Strömungsgeschwindigkeiten von $10 \mathrm{~m} / \mathrm{s}$ sehr ungenau werden. Da in dieser Arbeit gerade Geschwindigkeiten von maximal $5 \mathrm{~m} / \mathrm{s}$ gemessen werden, ist diese Technik für den benötigten Anwendungsbereich ungeeignet.

Eine weitere Alternative ist die Laser-Doppler-Anemometrie (LDA). Diese Messmethode ist allerdings sehr aufwendig und kostenintensiv. Zudem muss die Strömung mit Partikeln, dem so genanntem „Seeding“ versehen werden, und es sind zusätzliche Schutzmaßnahmen aufgrund der starken Laserstrahlung notwendig.

Es werden Eindrahtsonden der Firma TSI verwendet (eine schematische Darstellung befindet sich in Abb. 4.1) [34]. Der Draht besteht aus Wolfram und hat einen Durchmesser von $5 \mu \mathrm{m}$. Mit der Eindrahtsonde kann bei bekannter Strömungsrichtung nur der Betrag einer eindimensionalen Strömung senkrecht zum Draht ermittelt werden. Es wird der Betrag der Strömung in der x-y-Ebene gemessen. Die z-Komponente wird vernachlässigt. Es wäre auch möglich mithilfe einer Zweidrahtsonde die x- und die yRichtung getrennt voneinander zu messen, allerdings liegt der Messbereich des Sensors nur in einem $\pm 45^{\circ}$ Kegel um den Sensor. Bei der Eindrahtsonde beträgt dieser $\pm 90^{\circ}$. Es ist daher nicht möglich eventuelle Rückströmungen zu messen.

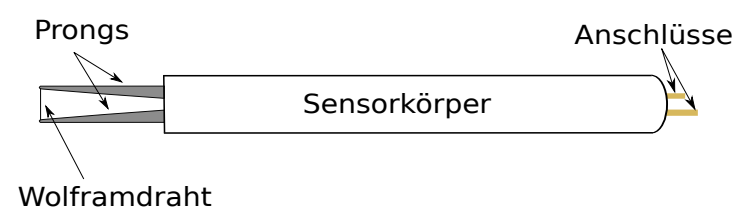

Abbildung 4.1.: Schematische Darstellung der verwendeten Ein-Drahtsonden.

Bei der Hitzdrahtanemometrie gibt es zwei unterschiedliche Arten von Regelkreisen. Zum einen die „Constant Current Anemometry (CCA)“, bei der der Sensor mit einem konstanten Strom beheizt wird. Durch die Anströmung des Sensors ändert sich der Widerstand und dadurch die am Sensor gemessene Spannung. Allerdings gibt es bei diesem System keine Temperaturkompensation und es hat eine schlechtere Frequenzauflösung im Gegensatz zur „Constant Temperature Anemometry“ (CTA), welche daher für diese Versuche verwendet wird.

Bei der CTA-Methode wird die Temperatur des Drahtes konstant gehalten, in dem der Widerstand konstant gehalten wird. Dies geschieht unter Verwendung einer Wheatstone'schen Brückenschaltung (Abbildung 4.2). Dadurch kann indirekt über die Spannung die Strömungsgeschwindigkeit bestimmt werden. Durch die hohe zeitliche Auflösung $(10 \mathrm{kHz})$ können ebenfalls turbulente Fluktuationen erfasst werden. 


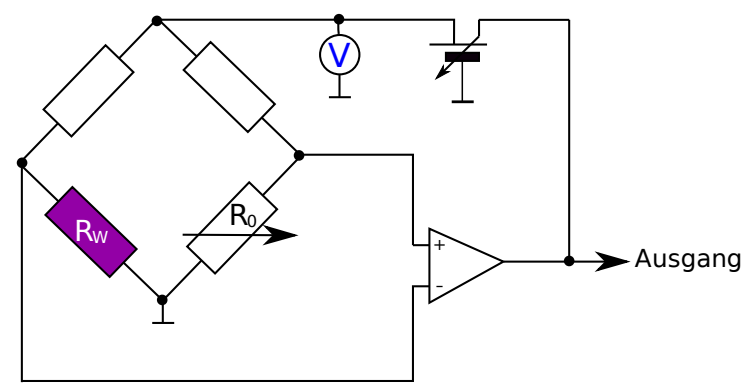

Abbildung 4.2.: Blockschaltbild CTA, der Widerstand des Drahtes $R_{w}$ wird auf $R_{0}$ abgeglichen.

\subsection{Kalibrierung}

Vor Beginn der Messung muss die Messbrücke abgeglichen und für die einzelnen Sensoren der Überhitzungsgrad und die Temperaturkorrektur eingestellt werden, dies wird automatisch von dem Programm StreamWare am PC berechnet, welches an das Messsystem angeschlossen ist. Anschließend erfolgt die Geschwindigkeitskalibrierung. Die Kalibrierung wird mit einer Kalibrierdüse durchgeführt. Die Ermittlung der Geschwindigkeit erfolgt über den Staudruck bzw. über den Druck in der Vorkammer der Düse. Aufgrund des quadratischen Zusammenhangs zwischen Druck und Geschwindigkeit (Bernoulli) ergeben sich für kleine Geschwindigkeiten die größten Unsicherheiten. Da das ausgegebene Spannungssignal nicht linear zu Strömungsgeschwindigkeit ist, wird für die Umrechnung das King'sche Gesetz verwendet [35]:

$$
I^{2} \cdot R_{w}=\left(T_{w}-T_{F}\right) \cdot\left(A+B \cdot u^{0,5}\right) .
$$

Bei $I$ handelt es sich um die Stromstärke, bei $R_{w}$ um den Widerstand des Drahtes, $T_{w}$ die Temperatur des Drahtes, $T_{F}$ die Temperatur des Fluids, und $A$ und $B$ sind Konstanten, die von den physikalischen Randbedingungen abhängen, wie zum Beispiel Brückeneinstellungen und Überhitzungsgrad.

Für die Bestimmung der Anpassungsfunktion am $\mathrm{PC}$ von der Spannung $U$ in die Geschwindigkeit $u$ eignet sich folgende Variation des King'schen Gesetzes:

$$
U^{2}=A+B \cdot u^{n},
$$

wobei $n=0,45$ als Startwert gesetzt wird und $A$ und $B$ variabel sind. Abbildung 4.3 zeigt die Kalibrierung eines Hitzdrahtsensors und die Anpassungskurve. Vor jeder neuen Messung muss der Hitzdraht erneut kalibriert werden.

\subsection{Winkelkalibrierung}

Mit der Eindrahtsonde ist es nur möglich, den Betrag der Geschwindigkeit zu messen. Aus der Potentialtheorie ist das theoretische Strömungsfeld bekannt und der Winkel 


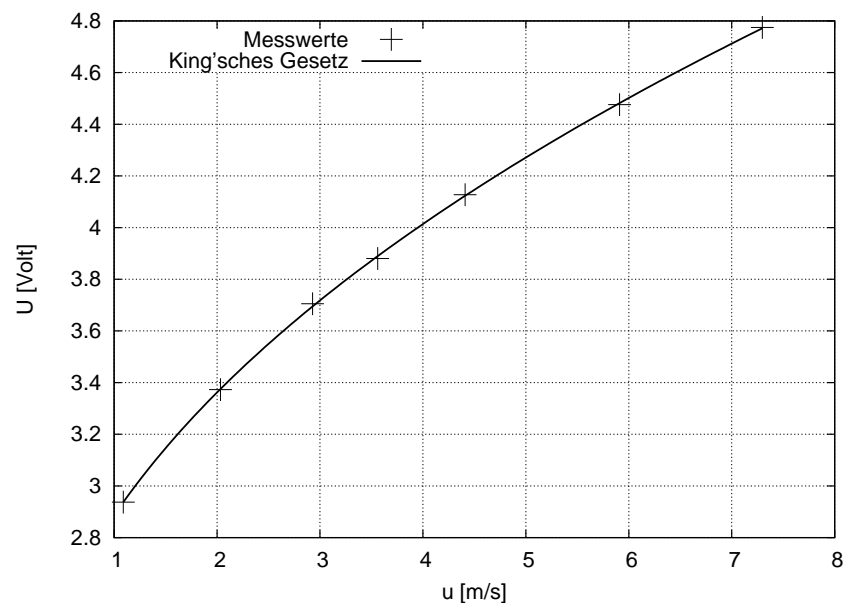

Abbildung 4.3.: Geschwindigkeitskalibrierung der Hitzdrahtsonde mithilfe des King'schen Gesetzes.

der Strömung kann daraus berechnet werden. Daher werden für die Hitzdrähte Winkelkorrekturen vorgenommen. Diese werden in der TSG im Seitenwindmodul durchgeführt. Der Hitzdrahtsensor wird in einen Schrittmotor eingespannt, so dass bei einer definierten Geschwindigkeit verschiedene Winkel durchgefahren werden können. Da der Hitzdraht nur von vorne (hier bei $0^{\circ}$ ) maximal angeströmt wird, zeigt der Hitzdraht bei anderen Winkeln zu hohe Geschwindigkeiten an. Dieser Zusammenhang wird verwendet, um später mithilfe des theoretischen Winkels die tatsächliche Geschwindigkeit zu berechnen. Der theoretische Winkel der Strömung berechnet sich aus den Geschwindigkeiten in xund y-Richtung wie folgt:

$$
\Phi(x)\left[^{\circ}\right]=\operatorname{arccot}\left(\frac{v(x)}{u(x)}\right) \cdot \frac{180^{\circ}}{\pi}+90^{\circ},
$$

Die Addition von $90^{\circ}$ ist notwendig, damit der Definitionsbereich stimmt. In Abbildung 4.4 ist der theoretische Winkel über den Weg in x-Richtung aufgetragen.

Abbildung 4.5 zeigt den experimentellen Aufbau: $\mathrm{Zu}$ sehen ist die Eindrahtsonde eingespannt im Schrittmotor im Seitenwindmodul und die Drehrichtung. Es werden die Winkel $0^{\circ}-180^{\circ}$ in $\Delta 5^{\circ}$-Schritten durchgefahren bei $u_{\infty}=3,3 \mathrm{~m} / \mathrm{s}$. Die gemessene Korrekturkurve befindet sich in Abbildung 4.6. Die Messwerte wurden mithilfe eines Polynoms vierten Grades approximiert. Es ist zu erkennen, dass der Hitzdraht nur bis zu einem Anströmwinkel von max. $\Phi=120^{\circ}$ brauchbare Werte liefert. Bei größeren Winkeln ist der Halter im Weg und der Hitzdraht kann keine Rückströmungen mehr erfassen. Die Funktionsgleichung der Korrekturkurve, mit der die späteren Messwerte korrigiert werden, lautet wie folgt:

$$
u / u_{0}=1-(8,7 \pm 0,7) \cdot 10^{-5} \cdot \Phi^{2}+(5,8 \pm 0,3) \cdot 10^{-7} \cdot \Phi^{4}
$$




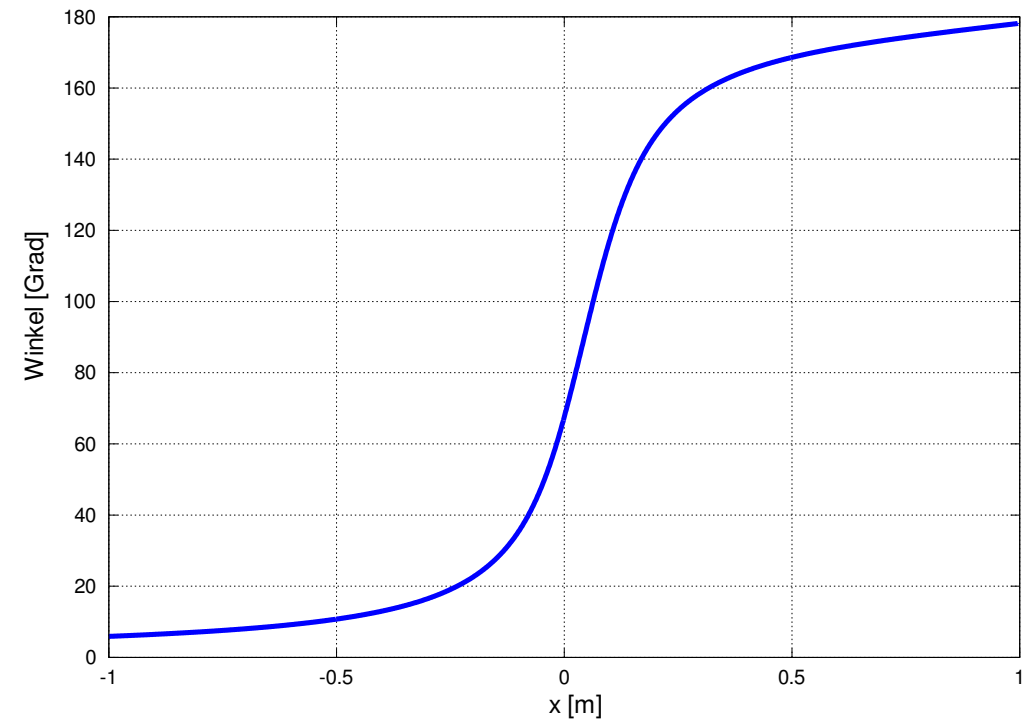

Abbildung 4.4.: Theoretischer Winkel der Strömung bei der Vorbeifahrt (Gl. 4.3). Die Stelle $x=0$ bezeichnet den Ort, an der sich die Zugnase auf Höhe der Hitzdrahtsonde befindet.

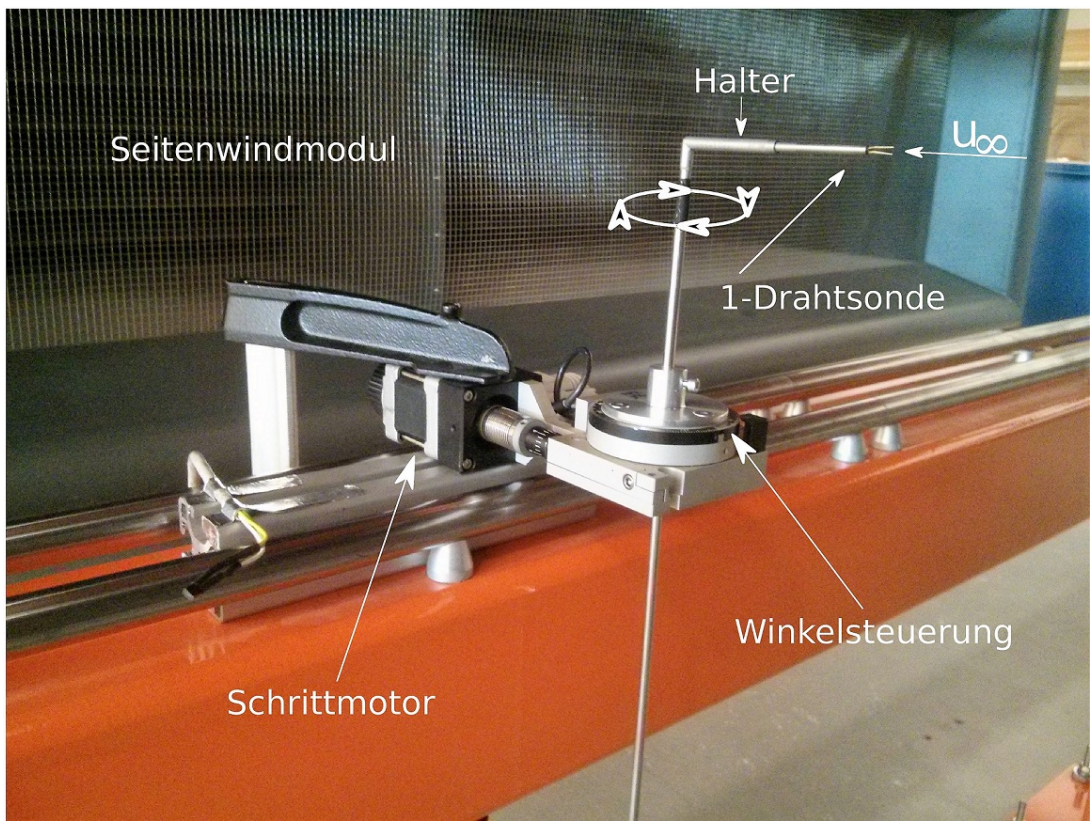

Abbildung 4.5.: Experimenteller Aufbau der Winkelkalibrierung der Hitzdraht Eindrahtsonde im Seitenwindmodul in der TSG. 


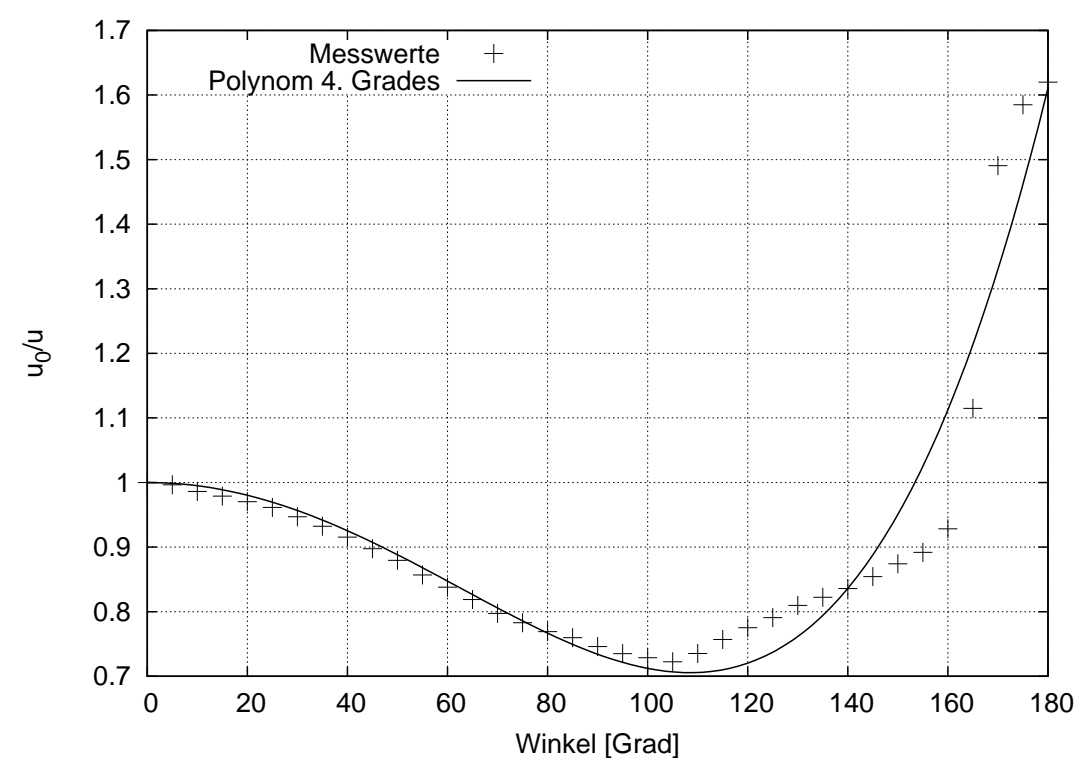

Abbildung 4.6.: Korrekturkurve für den Hitzdrähte in Fahrtrichtung (Hitzdraht 2 in Abb. 4.7) (Gl. 4.4). Für den Hitzdraht lateral zur Fahrtrichtung (Hitzdraht 1 in Abb. 4.7) wird die Kurve entsprechend um $90^{\circ}$ verschoben.

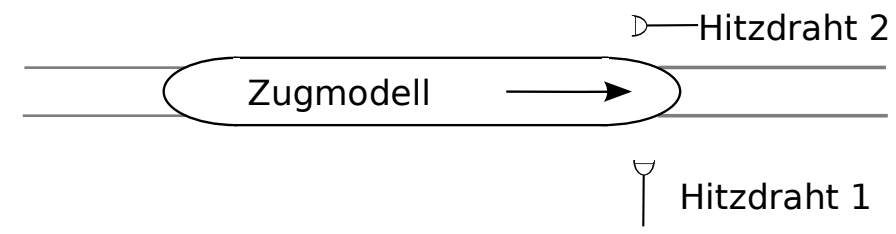

Abbildung 4.7.: Schematische Darstellung des Versuchsaufbau in der Messstrecke der TSG zur Geschwindigkeitsmessung mittels Hitzdrahtanemometrie.

\subsection{Aufbau und Durchführung der Vorbeifahrtmessungen}

Die Geschwindigkeitsmessungen werden in der TSG durchgeführt. Es wird das Potentialzugmodell und der flache Boden verwendet, da überprüft werden soll, ob das Strömungsfeld, welches vom Zugmodell erzeugt wird, dem theoretisch Berechnetem entspricht. Es werden zwei Eindrahtsonden verwendet. Eine befindet sich in Fahrtrichtung und eine ist lateral zur Fahrtrichtung orientiert (siehe Abb. 4.7). Die Sonde lateral zur Fahrtrichtung dient dazu, die Rückströmung zu erfassen, wozu die Sonde in Fahrtrichtung nicht in der Lage ist. Die Sonden werden auch in ihrer jeweiligen Hauptausrichtung kalibriert. Eine schematische Darstellung des Versuchsaufbaus befindet sich in Abbildung 4.7. Alle Fahrten werden bei $u_{\infty} \approx 40 \mathrm{~m} / \mathrm{s}$ durchgeführt. Es werden verschiedene Gleisabstände und Höhen über der Schienenoberkante untersucht. Die genauen Konfigurationen und Variationen können Tabelle 4.1 entnommen werden. 


\begin{tabular}{|c|c|c|c|c|}
\hline & \multicolumn{3}{|c|}{$y_{M}[\mathrm{~cm}]$} \\
\hline & & 10 & 12 & 15 \\
\hline & 4,5 & $\checkmark$ & $\checkmark$ & $\checkmark$ \\
\hline . & 6,0 & & $\checkmark$ & \\
\hline$\Sigma$ & 8,0 & & $\checkmark$ & \\
\hline & 10,0 & & $\checkmark$ & \\
\hline
\end{tabular}

Tabelle 4.1.: Messmatrix Hitzdrahtmessungen, $u_{\infty} \approx 40 \mathrm{~m} / \mathrm{s} . y_{M}$ ist die Entfernung der Ballmitte von der Gleismitte und $z_{M}$ die Position der Ballmitte über der Schienenoberkante.

\subsection{Auswertung und Diskussion}

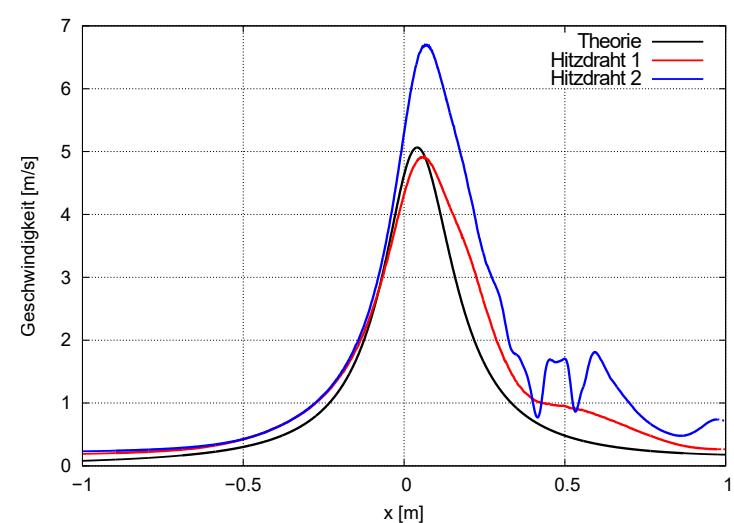

(a) Ohne Winkelkorrektur

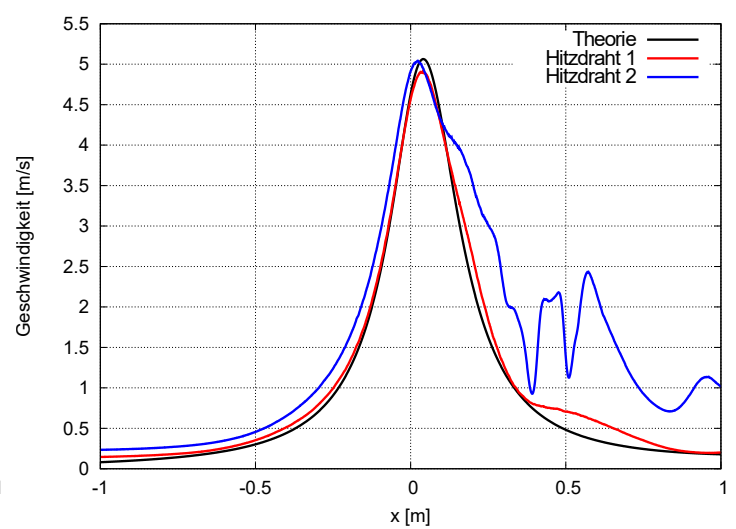

(b) Mit Winkelkorrektur

Abbildung 4.8.: Vergleich unkorrigiertes und korrigiertes Geschwindigkeitssignal für $y_{M}=10 \mathrm{~cm}, z_{M}=4,5 \mathrm{~cm}, u_{\infty}=40,48 \mathrm{~m} / \mathrm{s}$.

Abbildung 4.8 zeigt die gemessenen Geschwindigkeiten an der Position $y_{M}=10 \mathrm{~cm}$, $z_{M}=4,5 \mathrm{~cm}$ mittels Hitzdrahtanemometrie. Die Geschwindigkeitskurven (korrigiert und unkorrigiert) aller weiteren Messungen befinden sich in Anhang A. Auch diese Messungen zeigen sehr gute Übereinstimmungen zwischen gemessener und potentialtheoretisch berechneter Strömung. Die schwarze Kurve ist der theoretische Geschwindigkeitsbetrag in $\mathrm{x}$ - und $\mathrm{y}$-Richtung und berechnet sich wie folgt:

$$
\left|u_{x, y}(x)\right|=\sqrt{u(x)^{2}+v(x)^{2}} .
$$

Die blaue Kurve ist die gemessene Geschwindigkeit des Hitzdrahtsensors in Fahrtrichtung und die rote Kurve ist die gemessene Geschwindigkeit des Hitzdrahtsensors, welcher lateral zur Fahrtrichtung ausgerichtet ist.

Abbildung 4.8(a) zeigt die unkorrigierten Geschwindigkeitskurven. Der Hitzdrahtsensor 


\section{Geschwindigkeitsmessungen an der TSG mithilfe von Hitzdrahtanemometrie}

in Fahrtrichtung überschätzt das Geschwindigkeitssignal bei $\Phi=90^{\circ}$ um mehr als $35 \%$. Der Hitzdrahtsensor lateral zur Fahrtrichtung hingegen nicht, da dieser in genau diese Richtung kalibriert wurde.

Werden die Kurven nun mithilfe von Gleichung 4.4 korrigiert, stimmen beide gemessenen Geschwindigkeitskurven sehr gut mit der Theorie überein. Der Hitzdraht in Fahrtrichtung jedoch weicht bei $x=0,1 \mathrm{~m}$ ab, welches einem Anströmwinkel von $\Phi=120^{\circ}$ entspricht, ab welchem der Hitzdraht keine brauchbaren Werte mehr liefern kann. Für diesen Bereich ist dann der Hitzdraht lateral in Fahrtrichtung relevant, dieser misst den Rückströmungsbereich bis $x=0,4 \mathrm{~m}$ hinreichend genau. Diese Ergebnisse zeigen zum Einen, dass eine Winkelkorrektur notwendig ist, da die Geschwindigkeitsmessung sonst viel zu ungenau wäre. Gerade zu dem Zeitpunkt, wenn die Zugnase an den Sensoren vorbeifährt, also bei einem Anströmwinkel von $\Phi=90^{\circ}$, ist eine korrekte Geschwindigkeitsmessung wichtig, um genauere Vorhersagen für die Gefahr durch den vorbeifahrenden Zug auf Objekte in Gleisnähe treffen zu können. Zum Anderen, und das war der eigentliche Hintergrund für dieses Experiment, zeigen die gemessenen Geschwindigkeiten eine sehr gute Übereinstimmung mit der potentialtheoretischen Geschwindigkeit. Das heißt die Strömung um den Zug entspricht weitestgehend der potentialtheoretisch berechneten Zugumströmung. 
KAPITEL 5

Instationäre Kraftmessungen - Verwendete Messtechniken

Zur Messung der vom vorbeifahrenden Zug auf das Objekt induzierten Kraft werden zwei verschiedene Kraftmesstechniken verwendet, welche hier im Folgenden vorgestellt werden. Zum einen wird ein eindimensionales piezo-resistives Kraftmesselement verwendet, mit dem die induzierte Kraft direkt gemessen werden kann, zum Anderen wird eine Kraftmesstechnik basierend auf optischer Objektverfolgung verwendet.

\subsection{Eindimensionales Kraftmesselement}

Als Kraftmesselement wird ein piezo-resisitives Element verwendet, welches einer handelsüblichen Löffelwaage entnommen wurde. An dieses wird das Objekt, auf welches die Kräfte wirken sollen, befestigt. Es werden sehr kleine Kräfte gemessen, daher wird eine weiche Waage verwendet, welche jedoch eine niedrige Eigenfrequenz hat. Dies ist problematisch, da das Messsignal von der Dynamik der Waage entkoppelt werden muss. Ihre Berücksichtigung ist notwendig, um Kraftmessungen bei Frequenzen bis zur Eigenfrequenz der Waage durchführen zu können. Sie wird deshalb zunächst kalibriert.

\subsubsection{Kalibrierung des Kraftmesselements}

Bei der Kraftwaage handelt es sich um ein Masse-Feder-System, wobei sich die Masse aus der effektiven Masse der Feder und der Masse des Modells, also des Tischtennisballs, zusammensetzt. Durch die Vorbeifahrt wird auf den Tischtennisball eine Kraft ausgeübt, dadurch wird die Feder gedehnt bzw. gestaucht. Aus der Änderung der Federlänge kann schließlich die Kraft berechnet werden. Hier wird die Längenänderung mithilfe piezoresistiver Dehnmessstreifen gemessen, welche eine elektrische Spannung abhängig von ihrer Dehnung oder Stauchung ausgeben. Das resultierende Spannungssignal ist proportional zur Längenänderung der Feder. 


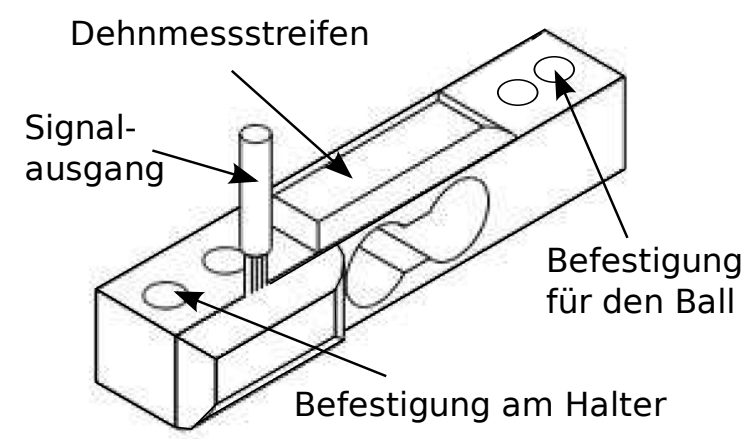

Abbildung 5.1.: Schematische Darstellung des Kraftmesselements.

Ein solches Masse-Feder-System wird durch folgende Differentialgleichung für die erzwungene gedämpfte Schwingung eines harmonischen Oszillators beschrieben:

$$
M \ddot{z}+R \dot{z}+D z=F(t) .
$$

Bei $z$ handelt es sich um die Auslenkung der Feder, $M$ die Masse, $R$ der Reibungskoeffizient und $D$ die Federkonstante. $\mathrm{F}(\mathrm{t})$ ist die anregende Kraft.

Da nicht direkt die Auslenkung, sondern eine Spannung $U$ gemessen wird, diese aber proportional zur Auslenkung ist, wird $z(t)$ durch folgenden Ausdruck ersetzt:

$$
\gamma U(t)=z(t),
$$

mit $\gamma$ als Proportionalitätsfaktor werden die folgenden Abkürzungen definiert:

$$
M_{*}=\gamma M, \quad R_{*}=\gamma R, \quad D_{*}=\gamma D .
$$

Die Differentialgleichung für die Spannung $U(t)$ lautet wie folgt:

$$
M_{*} \ddot{U}+R_{*} \dot{U}+D_{*} U=F(t) .
$$

Die Eigenfrequenz ergibt sich unter Annahme eines nicht gedämpften Systems ohne Reibung:

$$
M \ddot{z} \ll D z .
$$

Mit dem Ansatz:

$$
\begin{aligned}
z & =\epsilon \cos (\omega t), \\
\ddot{z} & =-\omega^{2} \epsilon \cos (w t) .
\end{aligned}
$$

gilt:

$$
\begin{gathered}
D \gg M \omega^{2}, \\
\frac{D}{M} \gg \omega^{2} .
\end{gathered}
$$


Unter der Annahme, dass die Eigenfrequenz $\omega_{0}$ größer ist als $\omega$, gilt:

$$
\begin{aligned}
& \omega_{0}^{2} \gg \omega^{2} \\
& \omega_{0}=\sqrt{\frac{D}{M}} .
\end{aligned}
$$

Entsprechend wird die Abklingkonstante $\beta$ eingeführt:

$$
\beta=\frac{R}{2 M} .
$$

Die homogene Differentialgleichung für den Fall ohne Anregung lautet:

$$
\ddot{U}=2 \beta \dot{U}+\omega_{0}^{2} U=0 .
$$

Diese kann mit dem Ansatz

$$
U(t)=\mathfrak{R e}\left(C e^{\xi t}\right)
$$

gelöst werden. Die charakteristische Lösung für $\xi$ ist:

$$
\xi^{2}+2 \beta \xi+\omega_{0}^{2}=0
$$

mit den Lösungen

$$
\xi_{ \pm}=-\beta \pm \sqrt{\beta^{2}-\omega_{0}^{2}} .
$$

Es wird im Folgenden $\omega_{0}>\beta$ angenommen. Das bedeutet, dass immer eine schwingende Lösung vorhanden ist und der aperiodische Grenzfall und der Kriechfall ausgeschlossen werden. Mit der Substitution

$$
\omega_{1}=\sqrt{\omega_{0}^{2}-\beta^{2}}
$$

kann

$$
\xi_{ \pm}=-\beta \pm i \omega_{1}
$$

geschrieben werden. Die Lösung der homogenen Differentialgleichung hat die Form:

$$
U(t)=\mathfrak{R e}\left\{\left[C_{+} e^{i \omega_{1} t}+C_{-} e^{-i \omega_{1} t}\right] e^{-\beta t}\right\},
$$

wobei die Konstanten $C_{+}$und $C_{-}$durch die Anfangsbedingungen bestimmt sind. Es kann auch eine gleichwertig reelle Lösung gefunden werden, diese lautet folgendermaßen:

$$
U(t)=A \cos \left(\omega_{1} t+\phi_{0}\right) e^{-\beta t} .
$$

Die Konstanten $A$ und $\phi_{0}$ ergeben sich entsprechend der Anfangsbedingungen.

Wird das System angeregt, können durch einen Fit des gemessenen Signals mit Gleichung 5.20 die beiden Größen $\omega_{1}$ und $\beta$ bestimmt werden, aus denen sich dann die Eigenfrequenz ohne Dämpfung ergibt:

$$
\omega_{0}=\sqrt{\omega_{1}^{2}+\beta^{2}} .
$$




\section{Instationäre Kraftmessungen - Verwendete Messtechniken}

Die Parameter der homogenen Differentialgleichung 5.13 sind nun bekannt. Die inhomogene Differentialgleichung 5.4 kann mithilfe der Abkürzungen wie folgt geschrieben werden:

$$
D_{*}\left[\left(\frac{\ddot{U}+2 \beta \dot{U}}{\omega_{0}^{2}}\right)+U\right]=F(t)
$$

Es sei

$$
\tilde{U}=\left(\frac{\ddot{U}+2 \beta \dot{U}}{\omega_{0}^{2}}\right)+U
$$

Daraus folgt:

$$
D_{*} \tilde{U}=F(t) .
$$

Dies beschreibt den Zusammenhang zwischen statischer Kraft und Spannung und kann durch Auflegen verschiedener Gewichte ermittelt werden.

Die anregende Kraft wird also in zwei Schritten rekonstruiert. Zunächst wird aus dem gemessenen Spannungssignal $U(t)$ mit Gleichung (5.23) $\tilde{U}$ bestimmt, dies ist das entfaltete Spannungssignal. Anschließend wird über Gleichung 5.24 aus dem entfalteten Spannungssignal $\tilde{U}$ die Kraft $F(t)$ berechnet.

\subsubsection{Filterung des Spannungssignals}

Aufgrund des hohen Rauschanteils, welcher sich hauptsächlich aus elektrischem und mechanischem Rauschen zusammensetzt, müssen die Signale zusätzlich gefiltert werden, da es bei der Entfaltung zu einer Verstärkung der Störsignale kommt. Die Auswertung der Signale geschieht im Frequenzraum, das Zeitsignal lautet:

$$
U(t)=\frac{1}{2 \pi} \int_{-\infty}^{\infty} U(\omega) e^{i \omega t} d \omega
$$

die Fourier-Transformierte lautet entsprechend:

$$
U(\omega)=\int_{-\infty}^{\infty} U(t) e^{-i \omega t} d t
$$

Die erste und die zweite zeitliche Ableitung in Integraldarstellung lauten wie folgt:

$$
\begin{gathered}
\dot{U}(t)=\frac{1}{2 \pi} \int_{-\infty}^{\infty} i \omega U(\omega) e^{i \omega t} d \omega \\
\ddot{U}(t)=\frac{1}{2 \pi} \int_{-\infty}^{\infty}-\omega^{2} U(\omega) e^{i \omega t} d \omega
\end{gathered}
$$

Mit Gleichung 5.23 ergibt sich:

$$
\tilde{U}(t)=\frac{1}{2 \pi} \int_{-\infty}^{\infty}\left[\frac{-\omega^{2}+i 2 \beta \omega}{\omega_{0}^{2}}+1\right] U(\omega) e^{i \omega t} d \omega .
$$


Es ergibt sich schließlich für die Fourier-Transformierte des Spannungssignals:

$$
\tilde{U}(\omega)=\left[\frac{-\omega^{2}+i 2 \beta \omega}{\omega_{0}^{2}}+1\right] U(\omega) .
$$

Mit der Fourier-Transformation des gemessenen Spannungssignals $U(t)$, Gleichung 5.31, und anschließender Rücktransformation wird das entfaltete Spannungssignal berechnet:

$$
\tilde{U}(t) \frac{1}{2 \pi} \int_{-\infty}^{\infty} \tilde{U}(\omega) \psi(\omega) e^{i \omega t} d \omega
$$

Hierbei handelt es sich bei $\psi(\omega)$ um eine Filterfunktion, die folgendermaßen definiert ist:

$$
\psi(\omega)=\left\{\begin{array}{lll}
1 & \text { für } & \omega /(2 \pi) \leq f_{a} \\
1-\frac{\left(\frac{\omega}{2 \pi}-f_{a}\right)}{\left(f_{b}-f_{a}\right)} & \text { für } & f_{a} \leq \omega /(2 \pi) \leq f_{b} \\
0 & \text { sonst } &
\end{array}\right.
$$

Bei $f_{a}$ und $f_{b}$ handelt es sich um die Eckfrequenzen, bei denen die Funktion $\psi(t)$ von $\psi\left(f_{a}\right)=1$ linear auf $\psi\left(f_{b}\right)=0$ fällt. In Abbildung 5.2 ist die Filterfunktion $\psi(\omega)$ graphisch dargestellt.

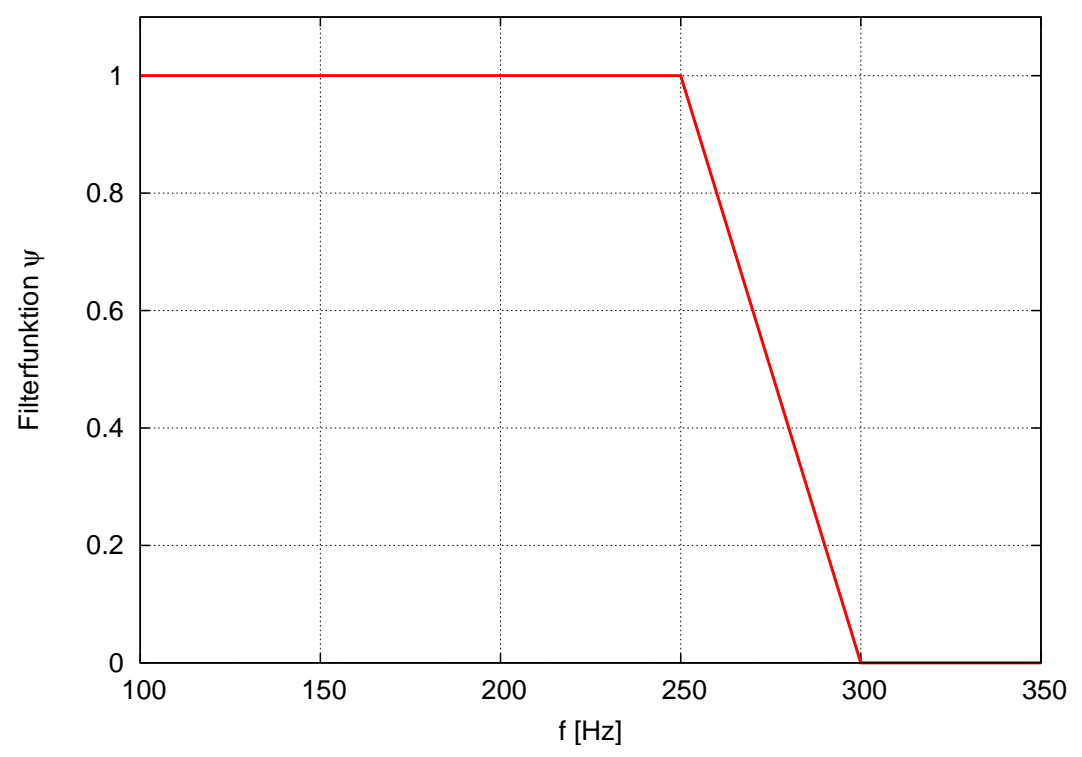

Abbildung 5.2.: Graphische Darstellung der Filterfunktion $\psi$.

Wenn nun die Auswertung am PC erfolgen soll, muss eine Diskretisierung des Integrals erfolgen. Die Zeitsignale sind bereits diskret, da sie zeitlich äquidistant mit einer bestimmten Abtastfrequenz aufgenommen wurden. Die diskretisierte Form von Gleichung 
5.32 ist also:

$$
\tilde{U}(t)=\frac{1}{N} \sum_{k=-\frac{N}{2}+1}^{N / 2} \tilde{U}_{k} \psi\left(\omega_{k}\right) e^{i \omega_{k} t},
$$

wobei $N$ die Anzahl der Abtastungen ist und $\omega_{k}=2 \pi \cdot \frac{k}{N} f_{U}$ ist. Hierbei bezeichnet $f_{U}$ die Abtastfrequenz.

Für die Fourier-Koeffizienten gilt dann analog zu Gleichung 5.31:

$$
\tilde{U}_{k}=\left[\frac{-\omega^{2}+i 2 \beta \omega}{\omega_{0}^{2}}+1\right] U_{k}
$$

Die Rücktransformation ist entsprechend definiert:

$$
U_{k}=\sum_{n=0}^{N-1} U\left(t_{n}\right) e^{i \omega_{k} t_{n}}
$$

mit $t_{n}=\frac{1}{f_{U}} n$ den äquidistanten Zeitpunkten. Die Hin- als auch die Rücktransformation werden mithilfe einer "Fast-Fourier-Transformation (FFT)" am PC durchgeführt.

Um die Entfaltung und Filterung der Signale durchführen zu können, müssen zunächst die Parameter $\beta$ und $\omega_{0}$ bestimmt werden. Dies geschieht mit der „dynamischen Kalibrierung". Dazu wird das Kraftmesselement leicht ausgelenkt und plötzlich losgelassen, es entsteht ein typisches Abklingsignal einer gedämpften Schwingung. Abbildung 5.3 zeigt die Abklingkurve des Löffelwaagenelementes in rot, mit der angepassten gefilterten Funktion (5.34) in grün und der entfalteten Kurve (5.23) in blau. Es ergeben sich die folgenden Werte für das Löffelwaagenelement: die Kreisfrequenz $\omega_{1}=(2456,73 \pm 0,31) \mathrm{s}^{-1}$ und die Abklingkonstante $\beta=(29,87 \pm 0,31) \mathrm{s}^{-1}$. Daraus ergibt sich nach (5.17) die Eigenfrequenz des reibungsfreien Systems $\omega_{0}=(2456,90 \pm 49,57) \mathrm{s}^{-1}$. Dies entspricht einer Frequenz von $(391,03 \pm 7,89) \mathrm{Hz}$.

Es ist zu erkennen, dass durch den Filter kleine Störungen entfernt werden. Die Entfaltung bewirkt, dass die abklingende Schwingung nicht mehr zu erkennen ist, sondern zeigt nur noch in dem Bereich ein Signal an, wo auch eine Kraft wirkt: bei der Auslenkung der Waage.

Die Kraft ergibt sich durch die „statische Kalibrierung“. Diese geschieht durch Anhängen verschiedener Gewichte an die Waage, die Spannung ist linear zur Ausdehnung des Dehnmessstreifens, also ergibt sich aus linearer Regression mit dem verwendeten 200-fachen Verstärker eine Umrechnungsfunktion für die Kraft:

$$
F(U(t))=(15,8 \pm 0,1)[\mathrm{N} / \mathrm{V}] \cdot U(t)[\mathrm{V}] .
$$

Bei den Abbildungen in 5.4 handelt es sich um eine exemplarische Auswertung für einen Vorbeifahrtversuch, mit der Geschwindigkeit $u_{\infty}=37,7 \mathrm{~m} / \mathrm{s}$, und einem Abstand von Kugelmitte zur Gleismitte von $y_{M}=12,5 \mathrm{~cm}$. Abbildung 5.4 (a) zeigt das unverarbeitete Spannungssignal der Waage in rot. Es sind noch sehr viele hohe Störfrequenzen enthalten, die durch die Filterung und Entfaltung entfernt werden (blaue Kurve). Zusätzlich 


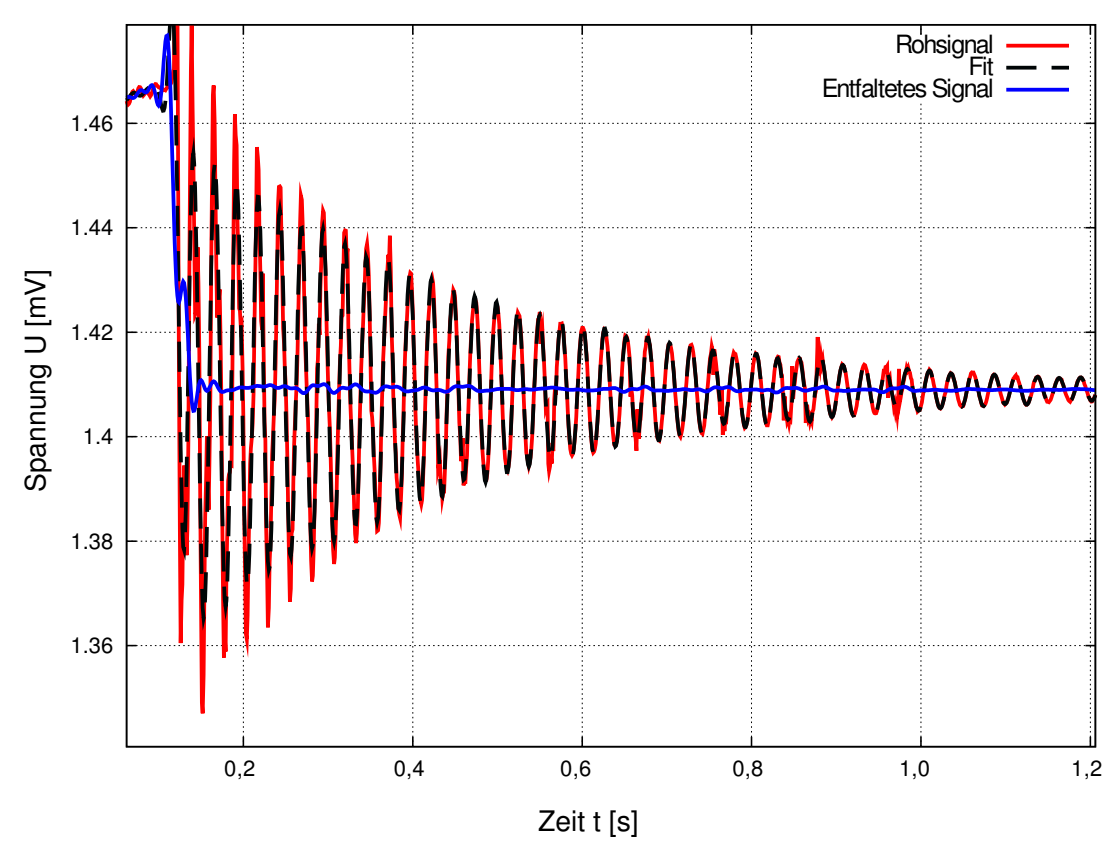

Abbildung 5.3.: Darstellung des Ergenbnisses der Signalverarbeitung. Das gemessene Signal $U(t)$ ist rot dargestellt, das gefilterte Signal in schwarz und das Ergebnis der Entfaltung $U(\omega)$ in blau.

zur oben beschriebenen Signalkorrektur wurde ein Beschleunigungssensor, exakt gegenüber der Waage, an der Halterung angebracht, um die Schwingung der Halterung weiter zu korrigieren. In Abbildung 5.4(b) ist zu erkennen, dass die Waage und der Beschleunigungssensor gleichphasig schwingen, es also möglich ist, $\tilde{U}(t)$ mithilfe des Beschleunigungssignals zu korrigieren. Dieses wird ebenfalls vorher gefiltert aber nicht entfaltet. So ergibt sich ein korrigiertes Signal:

$$
\tilde{U}_{k o r r}(t)=\tilde{U}(t)-\lambda \cdot a(t)
$$

Hierbei ist $\lambda$ ein Korrekturfaktor und $a(t)$ die Beschleunigung. $\lambda$ lässt sich aus den beiden Signalen folgendermaßen berechnen:

$$
\lambda=\frac{\tilde{U}(t) \circ a(t)}{a(t) \circ a(t)} .
$$

Abbildung 5.4(c) zeigt das unkorrigierte sowie das korrigierte Waagensignal. Die Beschleunigungskorrektur verringert das Rauschen merklich. Die Messzeit wird mithilfe der Signale aus den Lichtschranken in eine Strecke umgerechnet, bei $x=0 \mathrm{~m}$ befindet sich die Nase des Zugs exakt auf Höhe der Mitte des Tischtennisballs. 
5. Instationäre Kraftmessungen - Verwendete Messtechniken

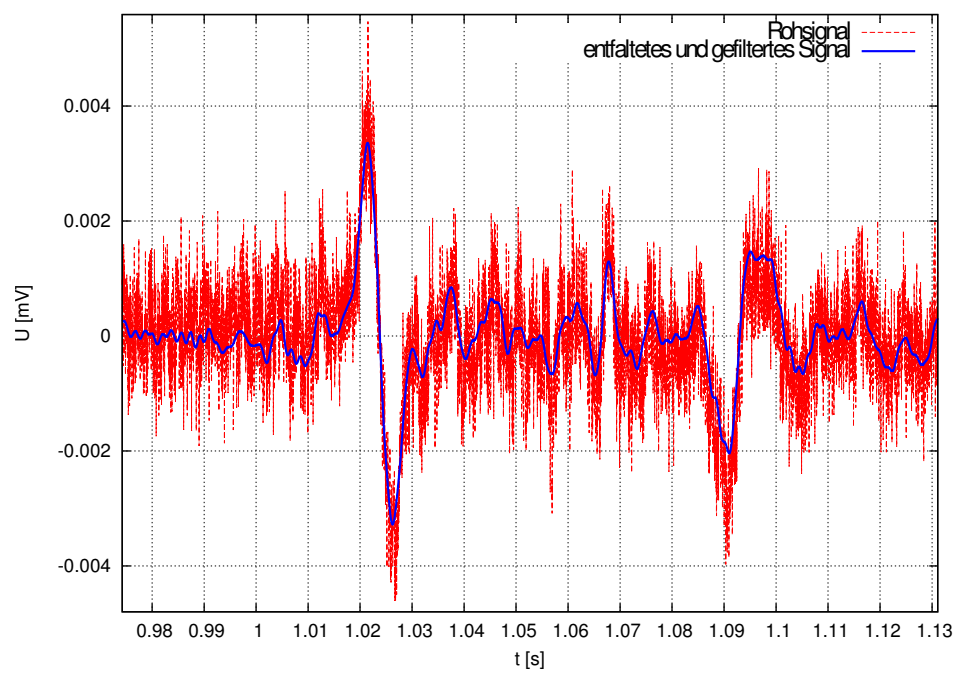

(a) Rohsignal (rot) und gefiltertes und entfaltetes Signal (blau).

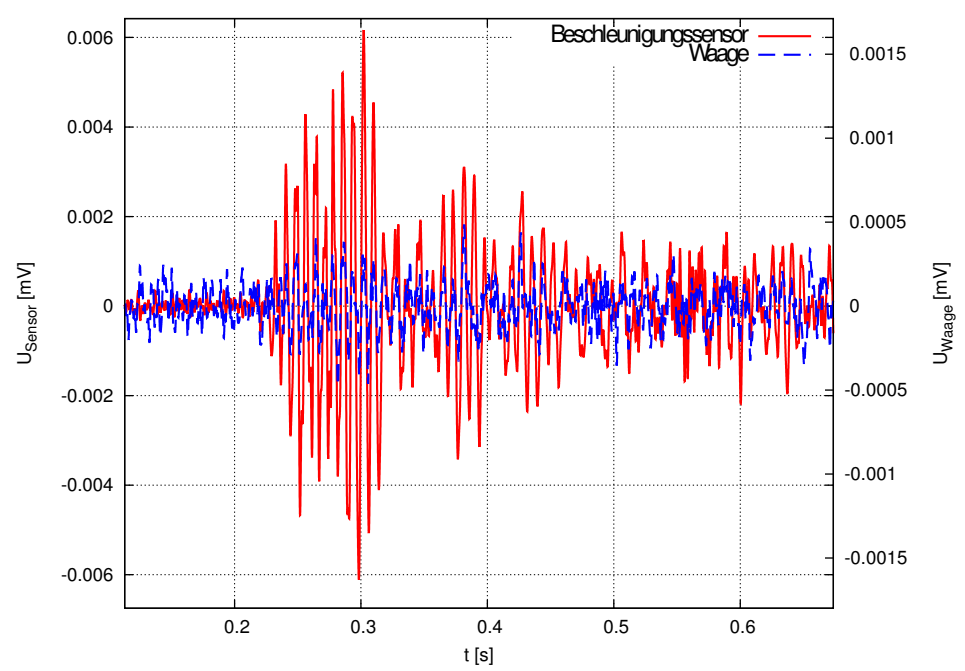

(b) Beschleunigungssensor- (rot) und Waagensignal (blau).

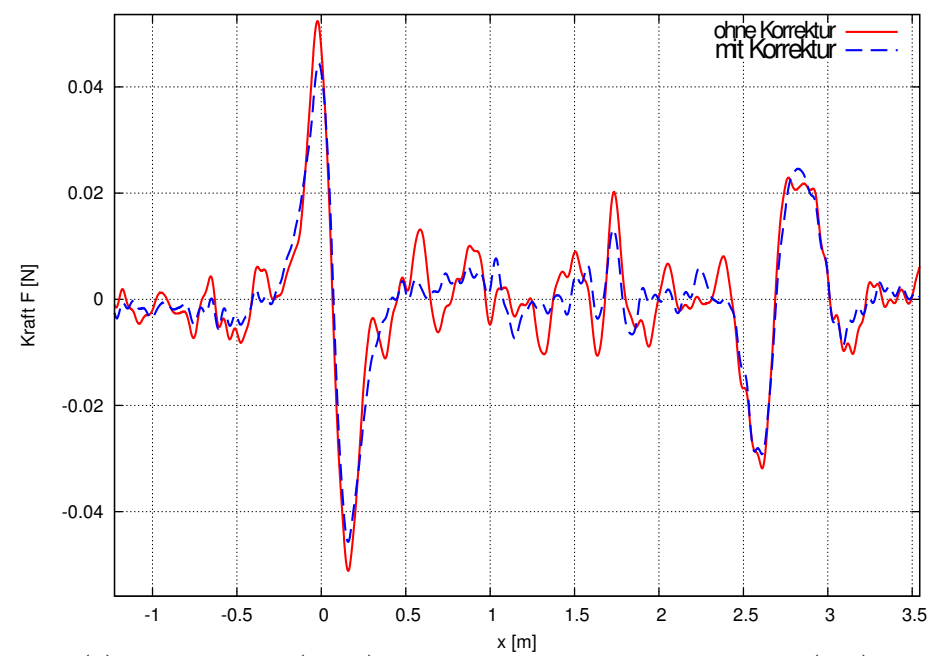

(c) Korrigierte (blau) und unkorrigierte Kraftkurve (rot).

Abbildung 5.4.: Exemplarische Auswertung für einen Vorbeifahrtversuch. 


\subsection{Instationäre Kraftmessungen mithilfe von optischer Objektverfolgung - Verwendeter Verfolgungsalgorithmus}

Zusätzlich zu den instationären Kraftmessungen mit einem eindimensionalem Kraftmesselement werden instationäre Kraftmessungen mithilfe von optischer Objektverfolgung durchgeführt. Diese haben den Vorteil, dass sie nichtinvasiv sind, die Kugel also, abgesehen von einem langen Faden, keine Befestigung braucht. Des Weiteren ist es möglich, die Bewegung in alle drei Raumrichtungen gleichzeitig zu verfolgen. Eine Phantom v1210 Hochgeschwindigkeitskamera [36] mit einem UV NIKKOR 105 mm 1:4,5 C-Mount Objektiv [37] wird verwendet, um die Bewegung der Kugel in einer Bildfolge aufzunehmen. Für diese Experimente wird die maximale Blende des Objektives von f/4,5 benutzt, um die maximale Helligkeit und maximalen Kontrast zu erhalten. Eine große Blende führt zudem zu einer niedrigeren Tiefenschärfe, was hier gewünscht ist, da die Kugel sich von Bild zu Bild bewegt, die Kontur aber auf jedem Bild so scharf wie möglich sein sollte. Die Aufnahmefrequenz beträgt $f=5000 \mathrm{~Hz}$. Die Aufnahmedauer beträgt $T=0,3 \mathrm{~s}$, was einer Anzahl von 1500 Bildern entspricht.

Die aerodynamischen Lasten werden aus der zweiten Ableitung der Objekttrajektorie multipliziert mit der Kugelmasse berechnet:

$$
\vec{F}=m \cdot \frac{\partial^{2} \vec{x}}{\partial t^{2}}
$$

Die Objekttrajektorie wird mithilfe eines iterativen Objektverfolgungsalgorithmus ermittelt. Der Algorithmus passt eine vorgegebene Objektgeometrie, in diesem Fall ein Kreis, auf die aufgenommenen Bilder an und liefert Werte für dessen Position, Winkel, Skalierung in jedem einzelnen Bild, so dass die Bewegung von Bild zu Bild berechnet werden kann. Bevor die Verfolgungsroutine gestartet werden kann, müssen für das erste Bild die Anfangsbedingungen für die freien Parameter vorgegeben werden. Diese sind die Fahrtrichtung $x$, die Richtung lateral zur Fahrtrichtung $y$ und einen Skalierungsfaktor $f_{S}$.

Die wesentlichen Schritte des Verfolgungsalgorithmus sind die Folgenden: Zunächst wird aus dem aufgenommen Bild ein Gradientenbild berechnet. Daher ist es bereits beim experimentellen Aufbau wichtig, eine ausreichende Hintergrundbeleuchtung und einen starken Kontrast zwischen Objekt und Hintergrund zu gewährleisten. Das Gradientenbild wird mit einer Kantendetektionsroutine, dem „Canny-Edge-Detektor“ ermittelt [38,39]. Zur Rauschreduktion wird das Bild zunächst mit einem Gauß-Filter geglättet. Im zweiten Schritt wird eine Sobel-Maske auf das Bild angewendet [40]. Hierbei wird der Gradient der Pixelintensität in horizontaler und vertikaler Richtung bestimmt. Abbildung 5.5 zeigt die verschieden Stufen des Algorithmus: Abbildung 5.5(a) das Originalbild, Abbildung 5.5(b) das Bild nach Anwendung des Sobel- und des Gauß-Filters, und Abbildung 5.5(c) zeigt wieder das Originalbild mit der gefundenen Kontur.

Die Modellkontur besteht zunächst aus den Pixeln mit einer Intensität oberhalb einer bestimmten Grenze. Diese werden für die weitere Behandlung markiert. Im nächsten 


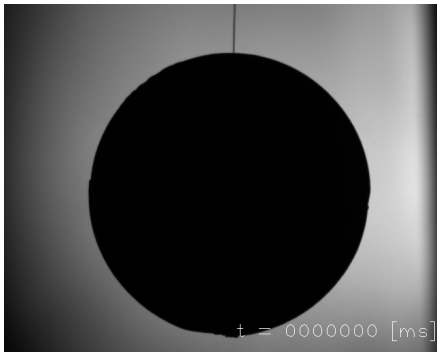

(a) Originalbild

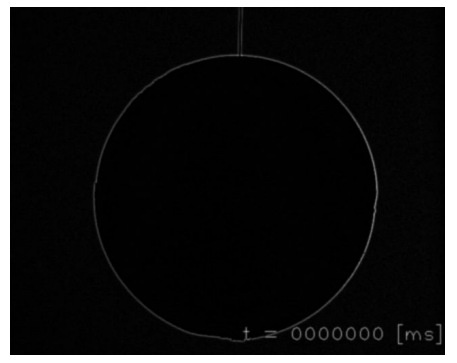

(b) Gradientenbild

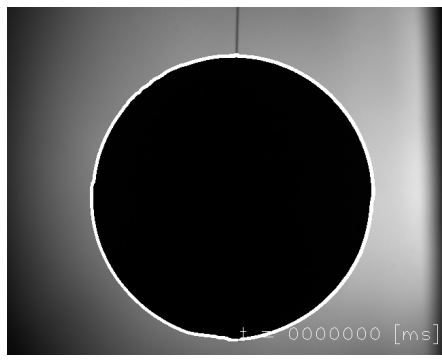

(c) Originalbild mit den gefundenen Pixeln für die Kontur

Abbildung 5.5.: Demonstration der Kantendetektion anhand eines Beispielbildes.
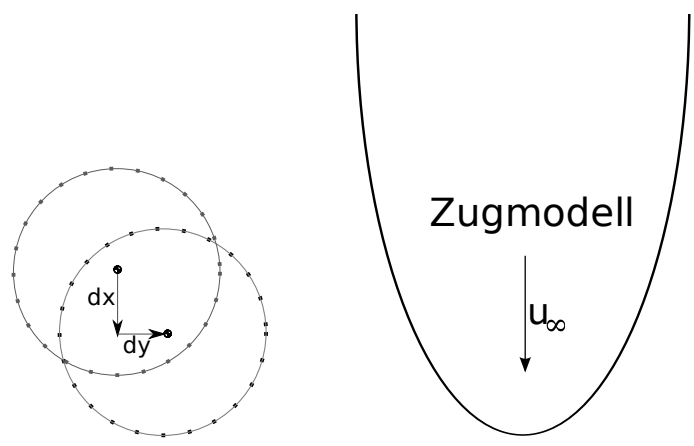

Abbildung 5.6.: Schematische Darstellung der Verfolgung der Kugel während der Vorbeifahrt des Zuges. Die gepunktete Linie stellt die gefundenen Kantenpixel dar und die durchgezogene Linie die analytische Modellbeschreibung des Kreises.

Schritt wird die gefundene Kontur optimiert. Um die beste Objektkontur zu erhalten, wird ein Levenberg-Marquardt-Löser [41] angewandt, welcher die Anfangsparameter $x, y$ und $f_{S}$ solange variiert, bis ein Optimum erreicht ist. Dabei werden alle markierten Pixel mit ihren acht jeweiligen Nachbarn verglichen. Pixel, welche außerhalb der optimierten Kontur liegen, werden anhand einer benutzerdefinierten Grenze aussortiert. Diese Prozedur wird für alle aufgenommenen Bilder wiederholt, wobei die zuletzt erhaltenen Parameter $x, y$ und $f_{S}$ des vorherigen Bildes als neue Anfangsparameter für das jeweils nächste Bild verwendet werden. Schließlich liegt für jedes aufgenommene Bild die jeweilige Position der Kugel vor, sodass die Trajektorie und daraus, mit der Masse der Kugel, die Kraft über die Zeit berechnet werden kann. Die Aufnahmefrequenz der Kamera ist ebenfalls bekannt, sodass auch jedes Bild einen Zeitstempel besitzt. 
Abbildung 5.6 zeigt schematisch die Verfolgung der Kugel während der Vorbeifahrt des Zuges. Die gepunktete Linie stellt die gefundenen Kantenpixel dar und die durchgezogene Linie die analytische Modellbeschreibung des Kreises. Aus den Vektoren $d x$ und $d y$ kann mithilfe der Aufnahmefrequenz der Kamera, der Zuggeschwindigkeit $u_{\infty}$ und der Masse der Kugel schließlich die wirkende Kraft berechnet werden. Dies geschieht, in dem der Radius als Umrechnungsfaktor verwendet wird. Der Radius in mm ist bekannt, und der Radius im Bild kann in Pixeln bestimmt werden. So erhält man den Umrechnungsfaktor $\mathrm{Pixel} / \mathrm{mm}$ und kann daraus die Länge der Vektoren $d x$ und $d y$ in $\mathrm{cm}$ bestimmen. 

KAPITEL 6

Instationäre Kraftmessungen an der TSG

\subsection{Instationäre Kraftmessungen mit einem eindimensionalen Kraftmesselement: ICE3 mit Bahndamm}

\subsubsection{Aufbau und Durchführung}

Um zu überprüfen, ob die instationäre Kraftmessung mit einem 1D-Kraftmesselement funktioniert, werden Vorversuche mit dem dreiteiligen ICE3-Modell, einem Tischtennisball als Objekt und einem Kraftmesselement, welches aus einer handelsüblichen Löffelwaage entnommen wurde, durchgeführt. Zudem ist noch ein Bahndamm installiert, welcher sich vom flachen Boden in der Theorie unterscheidet. Abbildung 6.1 zeigt den Versuchsaufbau mit dem Löffelwaagenelement, dem Tischtennisball und dem dreiteiligen ICE3-Modell im Maßstab 1:25. Wenn das Modell vom Katapult abgeschossen wird, entstehen Vibrationen, welche sich durch den Hallenboden ausbreiten und in den Versuchsaufbau hinein propagieren können. Daher ist das Kraftmesselement an einem Arm befestigt, welcher wiederum an auf dem Boden stehenden Aluminium Profilen befestigt ist. Zusätzlich ist der Arm, an dem die Waage befestigt ist, mit Knetmasse umschlossen und er liegt nicht auf dem Messtisch auf, sondern schwebt leicht darüber, um weitere Resonanzen zu verhindern. Das Kraftmesselement ist an einen Transientenrekorder (Typ DEWE 801 der Firma Dewetron) mit 200-fachen Verstärker angeschlossen, da Kräfte im Millinewton-Bereich erwartet werden (vgl. Abschnitt 2.3).

In diesem Vorversuch werden zwei verschiedene Konfigurationen untersucht, zum Einen die Variation des Abstandes $\left(y_{M}=9 \mathrm{~cm} ; y_{M}=11 \mathrm{~cm} ; y_{M}=12,5 \mathrm{~cm}\right.$ und $\left.y_{M}=15 \mathrm{~cm}\right)$ der Kugel zum Gleis bei gleichbleibendem Radius $(R=2 \mathrm{~cm})$ und Zuggeschwindigkeit. Zum Anderen die Variation des Radius der Kugeln bei gleichbleibendem Gleisabstand $\left(y_{M}=11 \mathrm{~cm}\right)$ und Zuggeschwindigkeit. Da es nur Tischtennisbälle mit dem Radius 


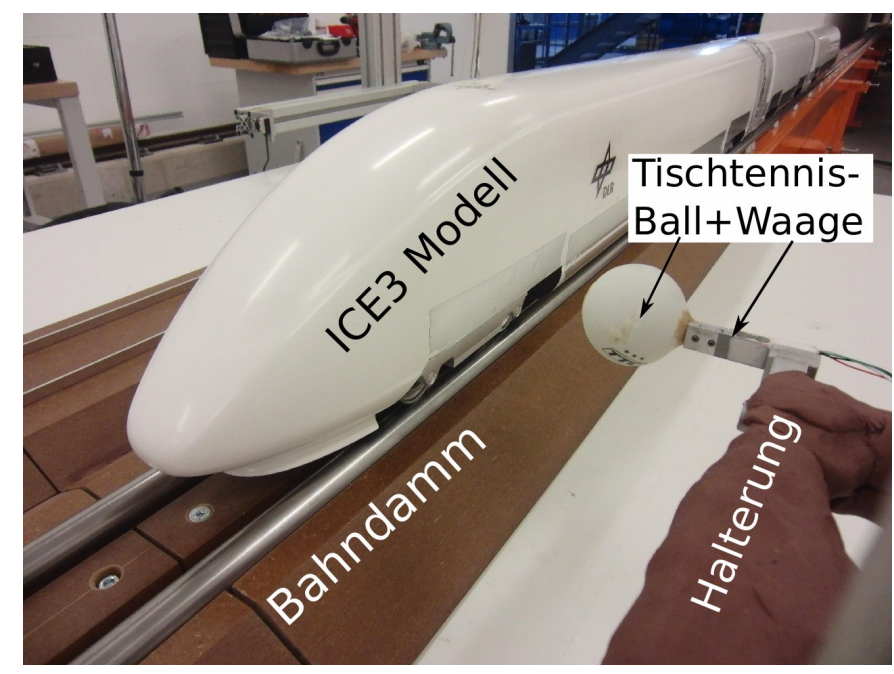

Abbildung 6.1.: Versuchsaufbau für die Kraftmessungen mit dem 1D-Kraftmesselement und dem ICE3-Modell an der TSG.

$R=2 \mathrm{~cm}$ gibt, werden hierfür Styropor-Bälle in den Größen $R=1,5 \mathrm{~cm} ; R=2 \mathrm{~cm}$; $R=2,5 \mathrm{~cm}$ und $R=3 \mathrm{~cm}$ verwendet. Bei allen Versuchen beträgt die Fahrtgeschwindigkeit des Zuges $u_{\infty}=37,8 \mathrm{~m} / \mathrm{s}$. Es wird erwartet, dass die Kopfwelle reproduzierbar ist, daher werden pro einzelner Konfiguration fünf Fahrten durchgeführt. Die Ergebnisse werden anschließend gemittelt, um sowohl das interne Rauschen der Waage zu reduzieren als auch externe Vibrationen, welche sich vom Katapult über den Boden und den Aufbau in die Waage ausbreiten können, zu vermindern. Da das Kraftmesselement die Kraft nur in eine Richtung messen kann, werden für die Kräfte in Fahrtrichtung (x-Richtung) und lateral zur Fahrtrichtung (y-Richtung) die Fahrten nacheinander durchgeführt, dabei wird das Kraftmesselement für die gewünschte Messung gedreht.

\subsubsection{Ergebnisse}

\section{Vergleich der Potentialtheorie mit den experimentellen Ergebnissen}

Abbildung 6.2 zeigt die gemittelte Kraft über fünf Fahrten bei der Vorbeifahrt des ICE3 auf den an dem Löffelwaagenelement befestigten Tischtennisball in x-Richtung (Abb. 6.2(a)) und in y-Richtung (6.2(b)) mit dazugehörigen RMS-Werten und im Vergleich dazu die potentialtheoretisch berechnete Kraft, wie sie von dem in Abschnitt 2.2.1 berechneten Zug ausgeübt werden würde.

Das Extremum bei $x=0$ ist die Kraft, welche durch den Bug des vorbeifahrenden Zuges ausgelöst wird, während das Extremum bei $x=2,7$ vom vorbeifahrenden Heck ausgelöst wird. Der Grund für die lokalen Extrema dazwischen sind die Wagenübergänge zwischen den einzelnen Wagen des ICE3-Modells.

Obwohl sich die potentialtheoretisch berechnete Geometrie und die im Experiment verwendete ICE3-Geometrie unterscheiden, stimmen die theoretischen und experimentel- 


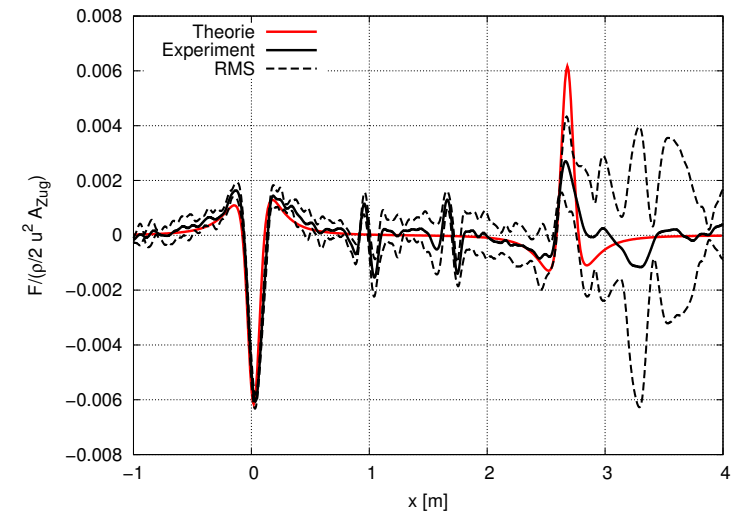

(a) x-Richtung

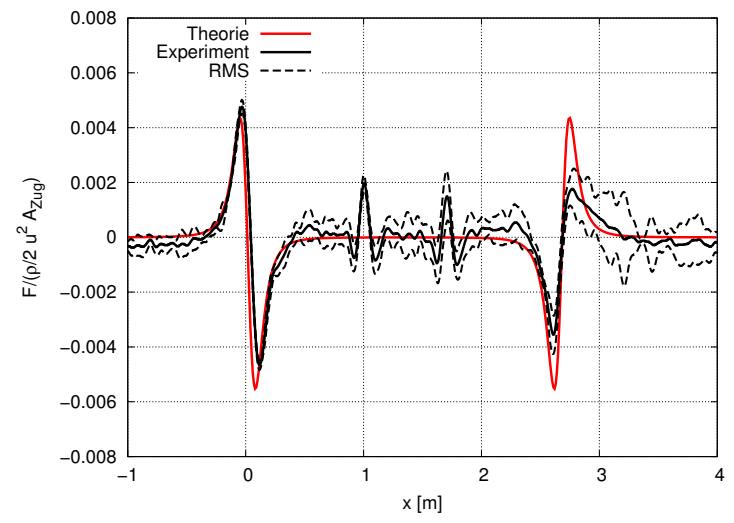

(b) y-Richtung

Abbildung 6.2.: Vergleich der gemessenen Kraft auf den Tischtennisball mit der potentialtheoretisch berechneten Kraft.

len Kräfte gerade im Bereich der Kopfwelle sehr gut überein. Der RMS-Wert ist sehr gering, was für eine gute Vorhersagbarkeit im Bereich der Kopfwelle durch die Potentialtheorie spricht. Hingegen sind beim Heck große Abweichungen zu beobachten und der RMS-Wert ist so groß, dass fünf Fahrten nicht ausreichen, um die mittlere Kraft ausreichend zu quantifizieren. Der RMS-Wert kann hier nicht mehr als Indikator für Messunsicherheit angewandt werden, sondern dient stattdessen als Indikator für turbulente Fluktuationen. Jene Fluktuationen sind in Fahrtrichtung größer als in quer zur Fahrtrichtung. Im Heckbereich sind große Reibungs- und Ablösungseffekte zu erwarten, sodass die Potentialtheorie hier nicht mehr angewandt werden kann. In den Abbildungen 6.2(a) und 6.2(b) ist zu sehen, dass der größte Kraftbeitrag tatsächlich durch die Kopfwelle erreicht wird. Da es bei der Vorhersagbarkeit der Kräfte auch um das maximale Gefährdungspotential geht, soll es im Folgenden nur noch um die Kräfte auf Objekte gehen, die durch die Kopfwelle des vorbeifahrenden Zuges ausgelöst werden, sodass die reibungsbehaftete Strömung des Hecks nicht mehr weiter betrachtet wird.

\section{Variation des Abstandes des Objektes zur Gleismitte}

Für die folgenden Vergleiche wird der Betrag $\Delta F$ verwendet. Dabei handelt es sich um den maximal ausgeübten Kraftbeitrag auf das Objekt $\Delta F=F_{\max }-F_{\min }$. In Abbildung 6.3 ist die Berechnung von $\Delta F$ graphisch dargestellt. Zur Untersuchung des Einflusses des Abstandes zwischen Objekt und vorbeifahrendem $\mathrm{Zug}$ werden vier verschiedene Gleisabstände variiert: $y_{M}=9 ; 11 ; 12,5 ; 15 \mathrm{~cm}$. Dabei bleibt der Radius der Kugel gleich bei $R=2 \mathrm{~cm}$ und die Fahrtgeschwindigkeit des ICE3-Modells bei $u_{\infty}=(37,9 \pm 0,1) \mathrm{m} / \mathrm{s}$ für jeweils fünf Fahrten je Konfiguration.

Abbildung 6.4 zeigt den normierten Kraftbeiwert $\Delta c_{F}=\Delta F /\left(0,5 \rho_{0} A_{\text {Zug }} u_{\infty}^{2}\right)$ aufgetragen über dem normierten Gleisabstand $y_{M} / D$ mit einem üblichen Zugdurchmesser von $D=12 \mathrm{~cm}$ verglichen mit der Theorie. Auch wenn die Geschwindigkeit von Messung zu 
6. Instationäre Kraftmessungen an der TSG

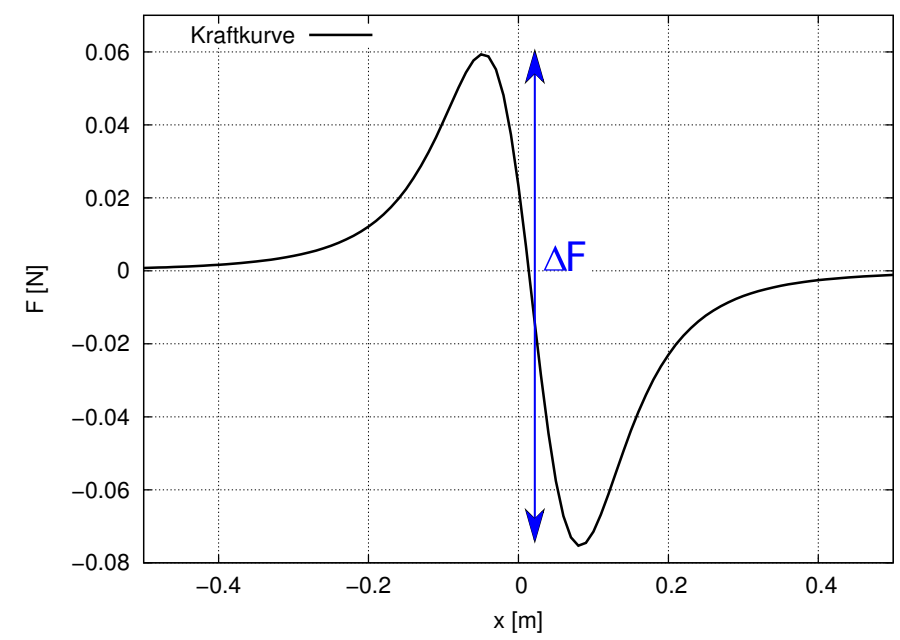

Abbildung 6.3.: Graphische Darstellung der Ermittelung von $\Delta F$.

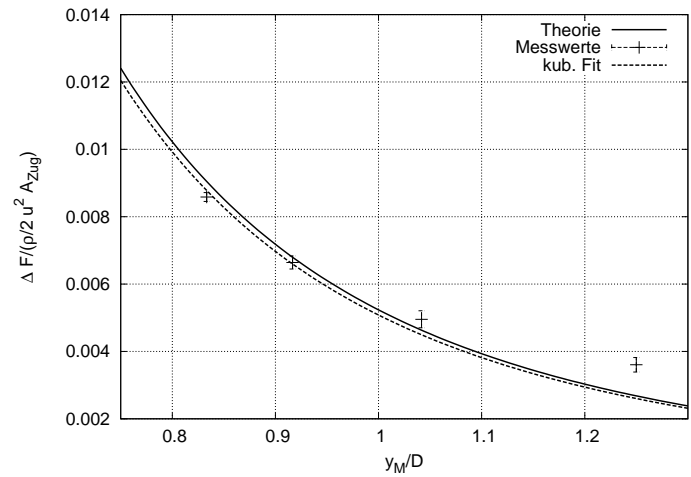

(a) x-Richtung

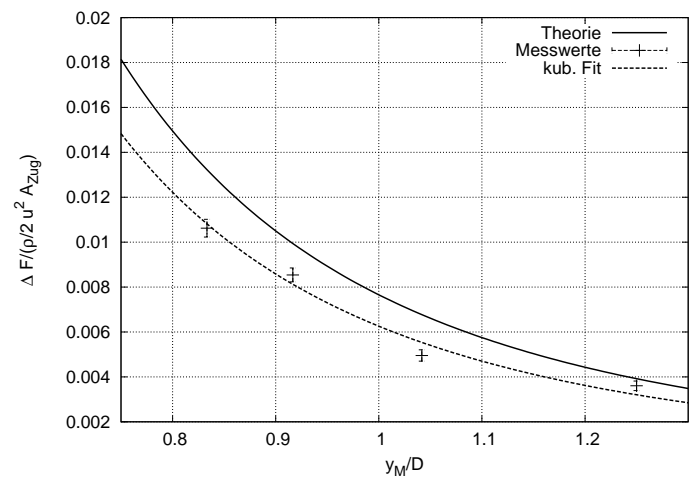

(b) y-Richtung

Abbildung 6.4.: Kräfte auf einen Tischtennisball $(R=2 \mathrm{~cm})$ bei verschiedenen Gleisabständen und einer Fahrtgeschwindigkeit von $u_{\infty}=37,9 \mathrm{~m} / \mathrm{s}$. 
Messung nur leicht schwankt, wird dieser Fehler durch die Normierung der Kraft durch den Kraftbeiwert herausgerechnet. Wie zu erwarten, nimmt die Kraft mit zunehmendem Gleisabstand ab. Die Kraft in Abhängigkeit vom Gleisabstand kann mit einer invers kubischen Funktion der Form $\Delta c_{F}=a \cdot\left(y_{M} / D\right)^{-3}$ approximiert werden. So ergeben sich für $\mathrm{x}$ - und $\mathrm{y}$-Richtung jeweils folgende Ergebnisse:

$$
\begin{aligned}
& \Delta c_{F_{x}}=(7,7 \pm 0,1) \cdot 10^{-3} \cdot\left(y_{M} / D\right)^{-3} \\
& \Delta c_{F_{y}}=(6,3 \pm 0,3) \cdot 10^{-3} \cdot\left(y_{M} / D\right)^{-3} .
\end{aligned}
$$

Das bedeutet, dass wenn der Abstand zwischen Objekt und Zug halbiert wird, bereits die achtfache Kraft auf das Objekt wirkt. Die Messwerte in Fahrtrichtung passen sehr gut mit den theoretischen Werten überein. Offensichtlich hat die Zuggeometrie hier keinen entscheidenden Einfluss auf die Kräfte, im Gegensatz zu den Kräften quer zur Fahrtrichtung. Dort fällt auf, dass sich die im Experiment verwendete Zuggeometrie von der potentialtheoretischen Geometrie unterscheidet. Gerade auch bei den kleineren Gleisabständen gewinnt die Zuggeometrie an Einfluss. Das ist zu erwarten, da Grenzschichteffekte vor allem im Nahfeld des Zuges einen größeren Einfluss auf die Kugel haben als im Fernfeld, wo Grenzschichteffekte keine Rolle mehr spielen und von einer annähernd laminaren Strömung ausgegangen werden kann. Des Weiteren ist die Verdrängungswirkung des ICE3-Modells eine andere als bei dem potentialtheoretischen Modell und das ICE3Modell hat Radkästen, in denen Verwirbelungen entstehen können. Diese Einflüsse fallen bei kleinen Gleisabständen mehr ins Gewicht als bei großen Gleisabständen.

\section{Variation des Kugelradius}

In Kapitel 2.3.2 wurde analytisch hergeleitet, dass instationäre Effekte hauptsächlich verantwortlich für die durch die Kopfwelle ausgeübte Kräfte auf eine Kugel und diese Kräfte proportional zum kubischen Radius der Kugel sind. Dieser Zusammenhang soll nun auch experimentell überprüft werden. Es werden Styropor-Bälle in den Größen $R=1,5 \mathrm{~cm} ; R=2 \mathrm{~cm} ; R=2,5 \mathrm{~cm}$ und $R=3 \mathrm{~cm}$ verwendet. Bei allen Versuchen beträgt die Fahrtgeschwindigkeit des Zuges $u_{\infty}=37,8 \mathrm{~m} / \mathrm{s}$. Es werden pro einzelner Konfiguration fünf Fahrten durchgeführt.

Abbildung 6.5 zeigt den normierten Kraftbeiwert $\Delta c_{F}=\Delta F /\left(0,5 \rho_{0} A_{\mathrm{Zug}} u_{\infty}^{2}\right)$ aufgetragen über dem normierten Radius $R / D$ mit einem üblichen Zugdurchmesser von $D=12 \mathrm{~cm}$ verglichen mit der Theorie bei einem Gleisabstand $y_{M}=0,11 \mathrm{~m}$ und einer Fahrtgeschwindigkeit von $u_{\infty}=37,8 \mathrm{~m} / \mathrm{s}$.

Sowohl die Kräfte in Fahrtrichtung, als auch die Kräfte quer zur Fahrtrichtung zeigen den erwarteten kubischen Zusammenhang zwischen Radius und Kraft. Die gemessenen und die theoretischen Kräfte zeigen eine sehr gute Übereinstimmung, obwohl die Zugmodelle unterschiedlich sind und in der Theorie ein flacher Boden und im Experiment ein Bahndamm verwendet wurde. Erst für größere Radien zeichnet sich eine leichte Abweichung ab. Diese liegt daran, dass je größer die Kugel wird, diese irgendwann nicht mehr als klein gegenüber dem Zugkopf angesehen werden kann und dann die Annahme 


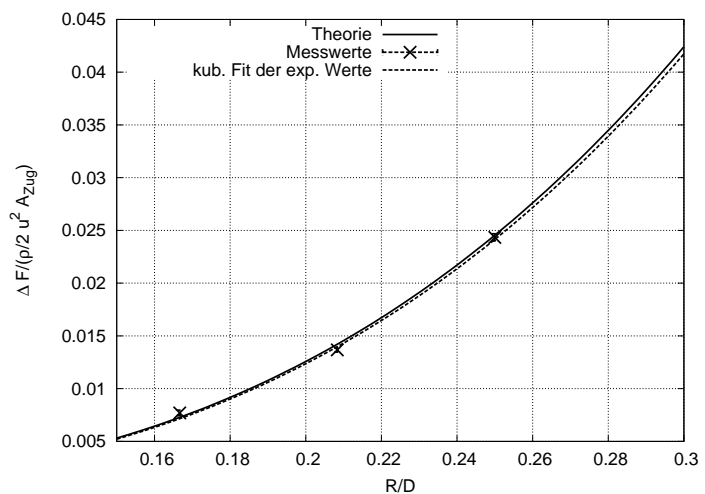

(a) x-Richtung

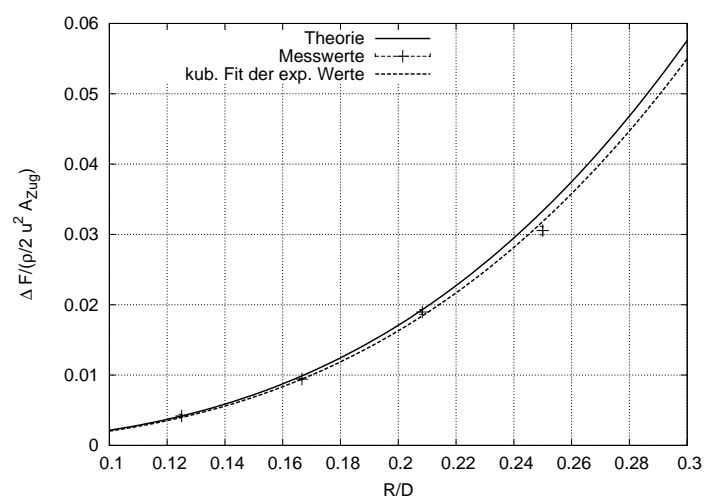

(b) y-Richtung

Abbildung 6.5.: Kräfte auf Styroporkugeln unterschiedlicher Radien bei einem Gleisabstand von $y_{M}=11 \mathrm{~cm}$ und einer Fahrtgeschwindigkeitkeit von $u_{\infty}=$ $37,8 \mathrm{~m} / \mathrm{s}$.

der ebenen Welle, die auf die Kugel trifft, nicht mehr zutrifft.

Aus der kubischen Approximation ergeben sich folgende Werte:

$$
\begin{aligned}
& c_{F_{x}}=(1,55 \pm 0,03) \cdot(R / D)^{3}, \\
& c_{F_{x}}=(2,04 \pm 0,03) \cdot(R / D)^{3} .
\end{aligned}
$$

\section{Fazit zu den Bahndammvorversuchen}

Die Vorversuche mit dem dreiteiligen ICE3-Modell und dem 1D-Kraftmesselement dienten dazu, zu überprüfen, ob die Messtechnik prinzipiell dazu geeignet ist, die potentialtheoretischen Vorhersagen zu validieren. Die Ergebnisse zeigen, dass die Waage, nachdem sie aufwendig kalibriert wird, durchaus in der Lage ist, die sehr kleinen instationären Kräfte von nur wenigen 100 Millinewton der Kopfwelle des vorbeifahrenden Zuges zu messen. Des Weiteren kann das Potentialmodell die Kräfte bereits sehr gut vorhersagen, obwohl die in den Experimenten verwendete Zuggeometrie nicht mit der potentialtheoretisch berechneten Geometrie übereinstimmt. Auch der Boden der Spiegelebene stimmt nicht überein, da in den Experimenten ein Bahndamm verwendet wurde. Aber auch dies hat keinen großen Einfluss auf die Ergebnisse. Dies ist insofern relevant, als das in der Realität häufiger Bahndämme oder andere Böden verwendet werden als flache Böden.

Für die nächsten Versuche werden die experimentellen Bedingungen weiter an die theoretischen Bedingungen angepasst, um weitere Fehlerquellen zu eliminieren, eine bessere Vergleichbarkeit mit der Potentialtheorie zu schaffen und um mögliche Grenzen der Potentialtheorie aufzeigen zu können. Vor allem wird statt des ICE3-Modells das potentialtheoretische Modell nachgebaut werden und dieses für die weiteren Experimente verwendet wird. 


\subsection{Exkurs: Iterative Anpassung der theoretischen Zugkontur anhand der experimentellen Ergebnisse für drei und fünf Quellen}

Obwohl die Ergebnisse aus dem vorherigen Abschnitt gezeigt haben, dass die gemessenen und die potentialtheoretischen Ergebnisse gute Übereinstimmungen zeigen, wird die Methode der kleinsten Fehlerquadrate verwendet, um die Unterschiede der Kraftfluktuationen in einem bestimmten Intervall zu minimieren. Dazu wurden in der potentialtheoretischen Simulation die Quellpositionen variiert, um eine ähnliche Nasenlänge wie die des ICE3 zu erhalten. Die Anpassung wird sowohl mit drei Quellen im Zugkopf, als auch mit fünf Quellen bei einem konstanten Gleisabstand von $y_{M}=15 \mathrm{~cm}$ durchgeführt.

Die Position der hintersten Quelle (in x-Richtung) wird solange in x- und z-Richtung iterativ variiert und die dazwischenliegenden Quellen äquidistant justiert, bis der Variationskoeffizient minimal ist:

$$
\vartheta=\sqrt{\frac{\sum_{t}\left(F_{\text {theo }}(t)-F_{\exp }(t)\right)^{2}}{\sum_{t}\left(F_{\exp }(t)\right)^{2}}} .
$$

Abbildung 6.6 zeigt die Kraftbeiwerte $c_{F}$ der verschiedenen Geometrien auf eine Kugel $(R=2 \mathrm{~cm})$ bei einem Gleisabstand von $y_{M}=15 \mathrm{~cm}$ in $\mathrm{x}$ - und in $\mathrm{y}$-Richtung aufgetragen über die normierte Strecke $x / D(D=12 \mathrm{~cm})$. Die angepassten Zugkonturen mit drei und mit fünf Quellen und die nicht angepasste Zugkontur mit drei Quellen werden mit den experimentellen Kraftbeiwerten, induziert durch das ICE3-Modell, verglichen. In Abbildung 6.7 ist zusätzlich das Polardiagramm dargestellt, in dem der Kraftbeiwert in y-Richtung $c_{F_{y}}$ über dem Kraftbeiwert in x-Richtung $c_{F_{x}}$ aufgetragen ist.

Da für die Anpassung der relative Fehler zwischen experimenteller und theoretischer Kurve minimiert wurde, ist zu erwarten, dass die Kräfte der angepassten Geometrien in besserer Übereinstimmung mit den experimentell gemessenen Kräfte sind als im Fall der nicht angepassten Geometrie. Dies ist vor allem für die Kräfte in Fahrt- bzw. x-Richtung der Fall. In y-Richtung, also quer zur Fahrtrichtung, wird vor allem der zweite Teil der Kraftkurve besser angepasst. Es besteht kein signifikanter Unterschied zwischen dem angepassten Dreiquellen- und dem angepassten Fünfquellenzug. Besonders im Polardiagramm wird deutlich, dass die Differenzen für die automatische Anpassung mit drei oder fünf Quellen sehr gering sind. Der Variationskoeffizient zwischen den theoretischen und experimentellen Kraftkurven $\vartheta$ ist für die verschiedenen Geometrien in Tabelle 6.1 notiert. Der Variationskoeffizient ist ein relativer Fehler und ein Maß für die Abweichung vom Erwartungswert, welcher hier die experimentelle Kurve ist. $\vartheta$ ist definiert als die Standardabweichung geteilt durch den Erwartungswert, und ist daher unabhängig von der Skalierung. Ein Wert von 0,2 bedeutet also, dass die Abweichung 20\% vom Erwartungswert ausmacht. 
6. Instationäre Kraftmessungen an der TSG

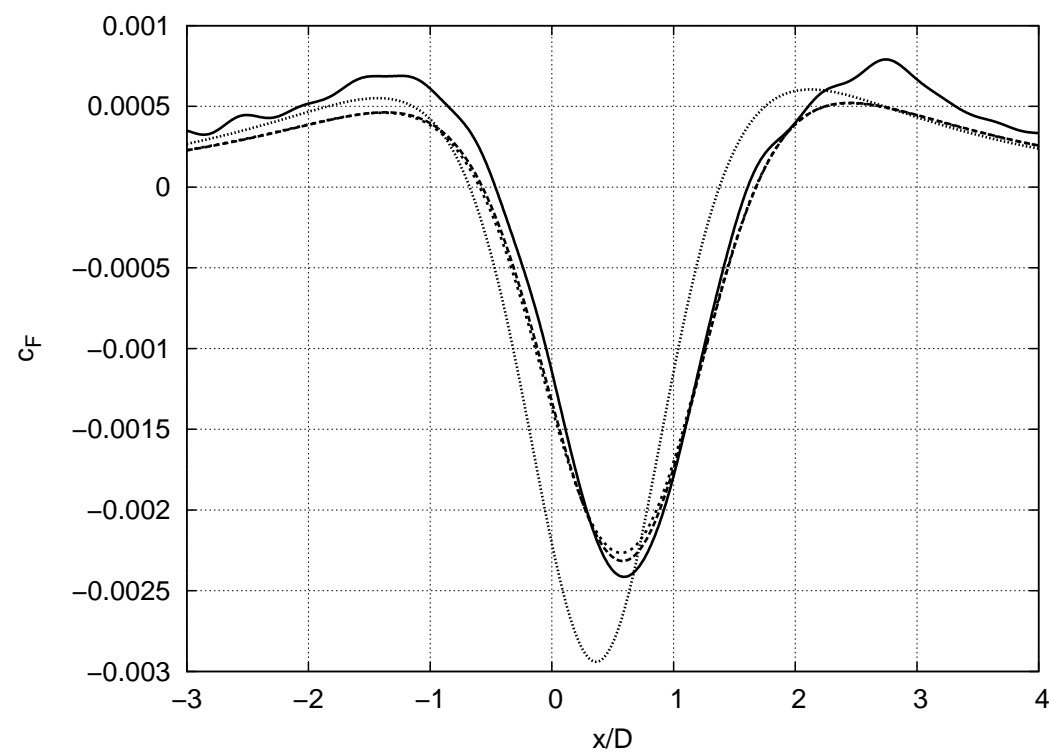

(a) x-Richtung

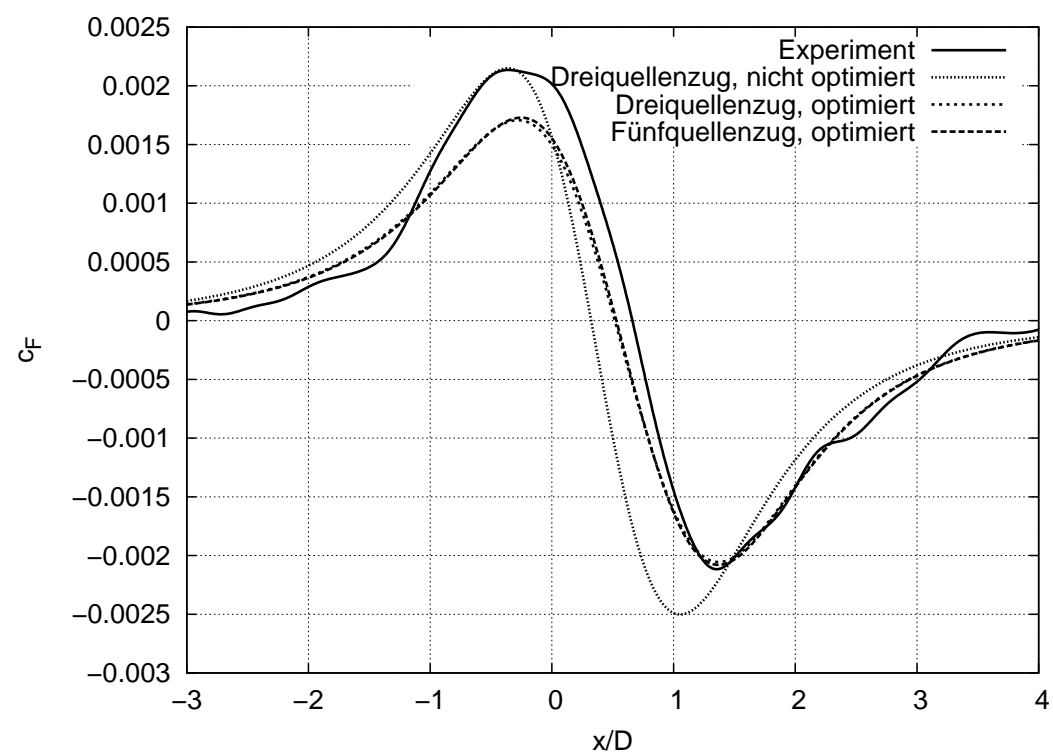

(b) y-Richtung

Abbildung 6.6.: Vergleich der Kräfte auf die Kugel für die angepasste und nichtangepasste Zuggeometrie sowie der experimentellen Kraftkurve. 
6.2. Exkurs: Iterative Anpassung der theoretischen Zugkontur

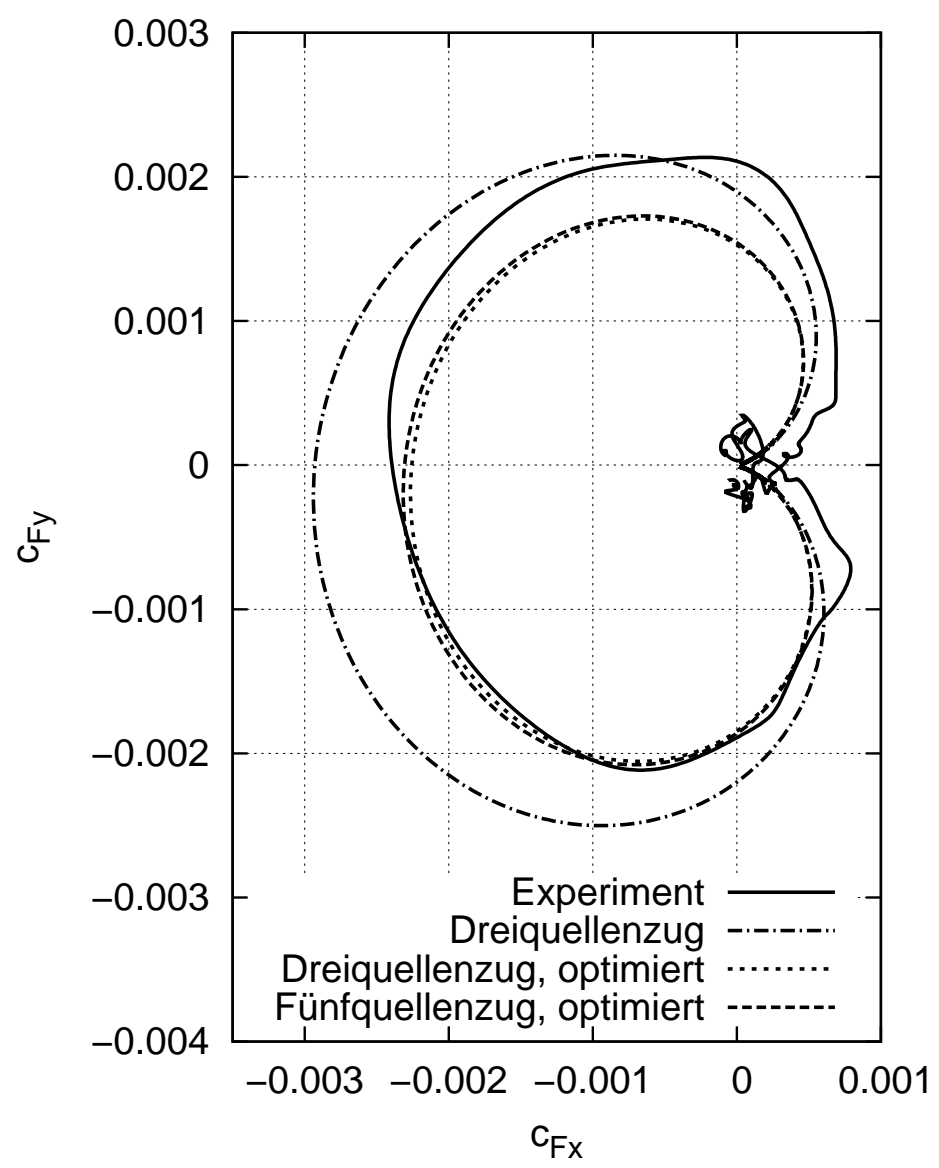

Abbildung 6.7.: Vergleich der Kräfte auf die Kugel für die angepasste und nichtangepasste Zuggeometrie sowie der experimentellen Kraftkurve als Polardiagramm. 


\begin{tabular}{l|c|c} 
Geometrie & $\vartheta_{x}$ & $\vartheta_{y}$ \\
\hline Dreiquellenzug, nicht angepasst & 0,41 & 0,49 \\
Dreiquellenzug, angepasst & 0,20 & 0,30 \\
Fünfquellenzug, angepasst & 0,19 & 0,29
\end{tabular}

Tabelle 6.1.: Variationskoeffizient $\vartheta$ zwischen den theoretischen und dem experimentellen Kraftverläufen in $\mathrm{x}$ - und in $\mathrm{y}$-Richtung für die verschiedenen Zuggeometrien (angepasst und nicht angepasst).

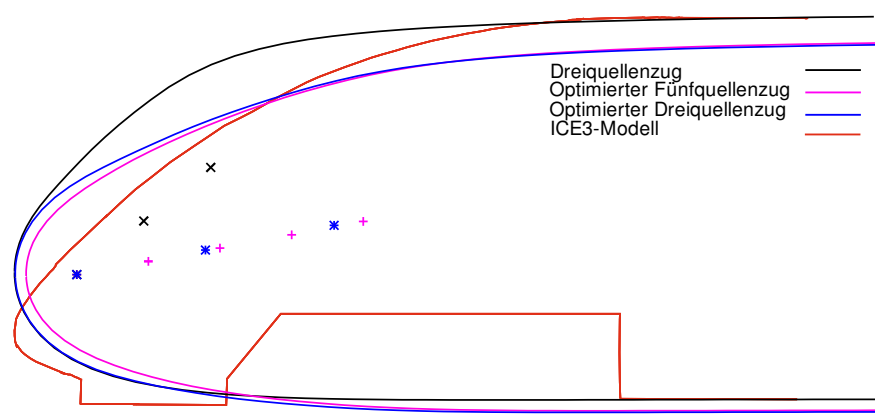

Abbildung 6.8.: Vergleich der angepassten Zugkonturen (drei bzw. fünf Quellen, blaue bzw. magentafarbene Linie) mit der nicht angepassten Zugkontur mit drei Quellen (schwarz) und der Kontur des ICE3-Modells (rot).

In Abbildung 6.8 sind die Konturen skizziert, welche sich aus der automatischen Anpassung ergeben. Die Nasenlänge der angepassten Zuggeometrien ist größer als beim nicht angepassten Zug und die Konturen ähneln mehr dem ICE3-Modell.

Es ist also möglich, mithilfe der Potentialtheorie auch kompliziertere Geometrien, wie die des ICE3, ansatzweise nachzubilden, indem die theoretischen Kräfte mit denen der Experimente verglichen werden, und so die Quellpositionen so lange variiert werden, bis der Fehler zwischen beiden minimal ist. Mit der angepassten Geometrie können dann die Kräfte auf die Kugel, noch besser vorhergesagt werden. Da es keinen signifikanten Unterschied macht, ob drei oder fünf Quellen für die Anpassung verwendet werden, können weiterhin nur drei Quellen verwendet werden.

\subsection{Instationäre Kraftmessungen mit einem eindimensionalen Kraftmesselement: Potentialzug mit flachem Boden}

\subsubsection{Aufbau und Durchführung}

Ziel der Versuche in der TSG ist es, die Potentialtheorie zu validieren, beziehungsweise zu untersuchen, in wie weit die Potentialtheorie dazu geeignet ist, die Kräfte auf Objekte, ausgelöst durch die Kopfwelle eines vorbeifahrenden Zuges vorherzusagen und herauszu- 


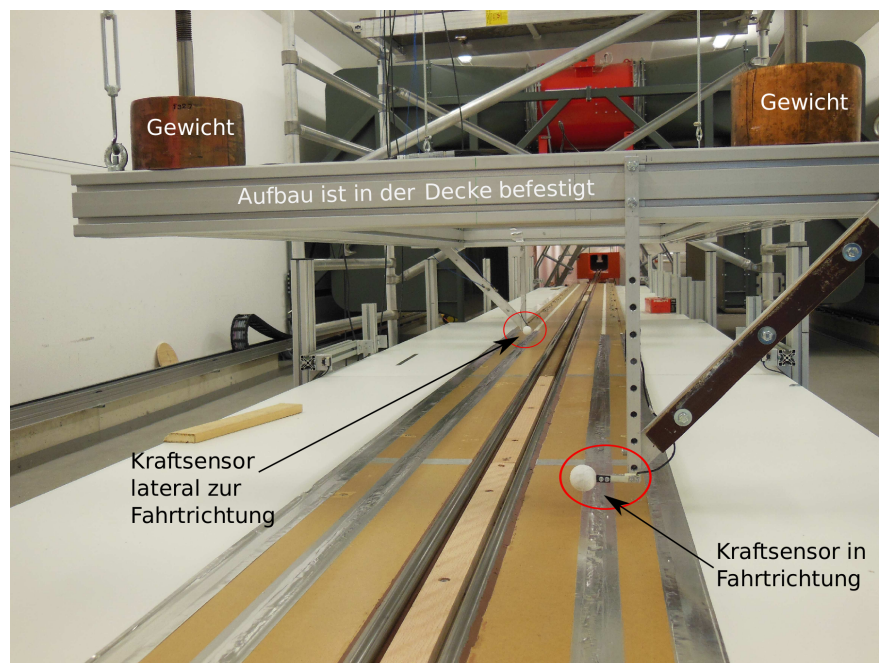

(a) Hängender Versuchsaufbau

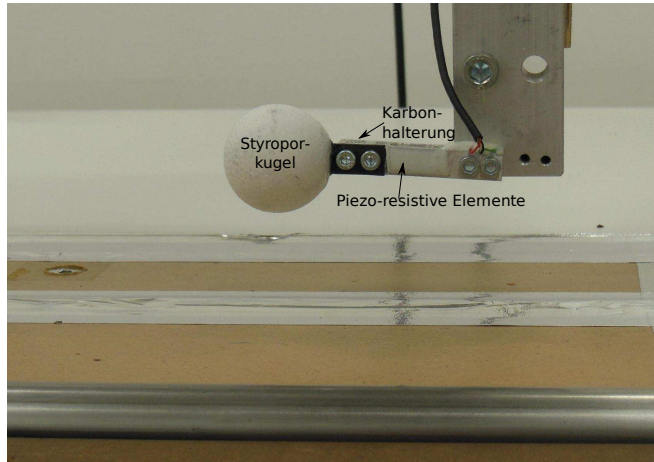

(b) Kraftmesselement mit Kugel

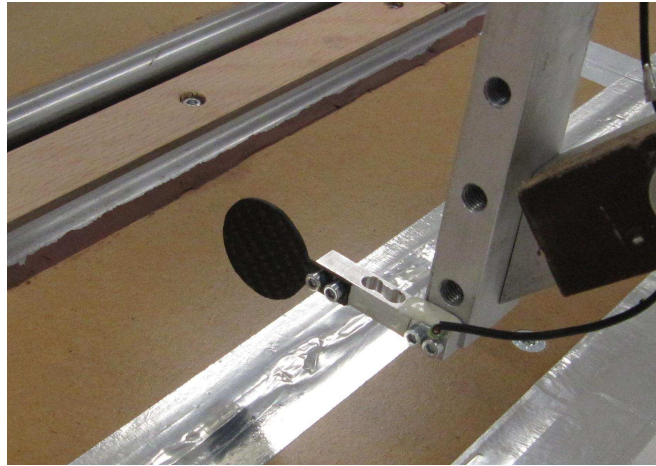

(c) Kraftmesselement mit Scheibe

Abbildung 6.9.: Versuchsaufbau zur instationären Kraftmessung in der TSG.

finden, wo ihre Grenzen liegen. Dazu sollte der experimentelle Aufbau dem theoretischem Aufbau möglichst exakt entsprechen. Statt des Bahndamms wird daher nun ein flacher Boden neben den Gleisen verwendet. Dieser repräsentiert die in der Theorie verwendete Spiegelebene. Als Modellzug wird in den folgenden Versuchen der Potentialzug verwendet, welcher aus dem theoretisch berechneten Dreiquellen-/Senken-Modell aus Kapitel 2.2.1 konstruiert wurde.

Für diese Versuchsreihe wurde der Versuchsaufbau weiter angepasst. Das Gestell, an dem die Waage befestigt ist, ist mit steifen Drahtseilen an der Decke befestigt, um die Schwingungen und Vibrationen, die durch das Katapult entstehen, und die durch den Boden propagieren, weiter zu minimieren. Das Gestell selbst ist wiederum zusätzlich mit Gewichten beschwert, damit es nicht schwingen kann. Des Weiteren können nun zwei Kraftmesselemente gleichzeitig verwendet werden, so dass die Kräfte in Fahrtrich- 


\begin{tabular}{|c|c|c|c|c|c|c|c|}
\hline & \multicolumn{4}{|c|}{$\boldsymbol{R}[\mathbf{c m}]$} & \multirow{2}{*}{$\begin{array}{c}\boldsymbol{y}_{\boldsymbol{M}}[\mathbf{c m}] \\
12\end{array}$} & \multirow{2}{*}{$\begin{array}{c}z_{M}[\mathbf{c m}] \\
4,5\end{array}$} \\
\hline & & 1,5 & 2,0 & 2,5 & 3,0 & & \\
\hline & 35 & \multirow{4}{*}{$\checkmark$} & $\checkmark$ & \multirow{4}{*}{$\checkmark$} & \multirow{4}{*}{$\checkmark$} & $\checkmark$ & $\checkmark$ \\
\hline छે & 40 & & $\checkmark$ & & & $\checkmark$ & $\checkmark$ \\
\hline$\frac{2}{8}$ & 45 & & $\checkmark$ & & & $\checkmark$ & $\checkmark$ \\
\hline$\frac{3}{8}$ & 50 & & $\checkmark$ & & & $\checkmark$ & $\checkmark$ \\
\hline $\bar{g}$ & 10 & \multirow{3}{*}{$\checkmark$} & $\checkmark$ & \multirow{3}{*}{$\checkmark$} & \multirow{3}{*}{$\checkmark$} & \multirow{3}{*}{$\checkmark$} & $\checkmark$ \\
\hline ن & 12 & & $\checkmark$ & & & & $\checkmark$ \\
\hline$\sum$ & 15 & & $\checkmark$ & & & & $\checkmark$ \\
\hline & 4,5 & \multirow[t]{4}{*}{$\checkmark$} & $\checkmark$ & \multirow[t]{4}{*}{$\checkmark$} & \multirow[t]{4}{*}{$\checkmark$} & $\checkmark$ & \multirow[t]{4}{*}{$\checkmark$} \\
\hline हृن & 6,0 & & $\checkmark$ & & & $\checkmark$ & \\
\hline$\Sigma$ & 8,0 & & $\checkmark$ & & & $\checkmark$ & \\
\hline$\hat{x}$ & 10,0 & & $\checkmark$ & & & $\checkmark$ & \\
\hline
\end{tabular}

Tabelle 6.2.: Messmatrix Potentialzug, Kugel.

tung und lateral zur Fahrtrichtung gleichzeitig gemessen werden können (eine Fotografie des Versuchsaufbaus befindet sich in Abb. 6.9(a)).

Als Objekte, auf welche die Kräfte des vorbeifahrenden Zuges induziert werden, werden in dieser Versuchsreihe Styroporkugeln und Karbonscheiben verschiedener Radien verwendet (siehe Abb.6.9(b) und 6.9(c)). Die Karbonscheiben haben eine Dicke von $d=0,5 \mathrm{~mm}$. Als Kraftmesselement wird ein $20 \mathrm{~N}$ Element KD45 der Firma MEMeßsysteme verwendet (vgl. Abb. 5.1). Dieses ist so steif, dass die Signalentfaltung, welche in Abschnitt 5.1 hergeleitet wurde, bei der Auswertung dieser Versuche, zumindest für kleine Kugeln, wie sie hier verwendet werden, nicht mehr notwendig ist. Bei der Auslenkung des Elements entsteht keine Abklingkurve mehr. Lediglich die Filterfunktion $\psi(\omega)$ wird weiter verwendet, um die Rohsignale zu filtern.

Es sollen die Einflüsse der aerodynamischen Lasten in Abhängigkeit von der Geometrie und der Position des Objektes untersucht werden. Dazu werden verschiedene Konfigurationen untersucht: verschiedene Objektradien, verschiedene Fahrtgeschwindigkeiten, verschiedene Abstände des Objektes zum Gleis und verschiedene Höhen des Objektes über der Schienenoberkante (Messmatrix für die Kugeln siehe Tabelle 6.2, für die Scheiben siehe Tabelle 6.3). Pro Bedingung werden jeweils fünf Fahrten durchgeführt.

Im Anschluss werden zur Untersuchung des Einflusses verschiedener Zuggeometrien auf die Kräfte auf die Kugel bei einer Bedingung die drei verschiedenen Zugmodelle, welche in Abschnitt 3.2 vorgestellt wurden, eingesetzt. 
6.3. Kraftmessungen mit einem Kraftmesselement: Potentialzug mit flachem Boden

\begin{tabular}{|c|c|c|c|c|}
\hline & \multicolumn{3}{|c|}{$\boldsymbol{R}[\mathbf{c m}]$} & \multirow{2}{*}{$\begin{array}{c}z_{M}[\mathbf{c m}] \\
4,5\end{array}$} \\
\hline & 1,5 & 2,0 & 2,5 & \\
\hline $\begin{array}{ll}\text { छ } & 10\end{array}$ & & $\checkmark$ & & $\checkmark$ \\
\hline 12 & $\checkmark$ & $\checkmark$ & $\checkmark$ & $\checkmark$ \\
\hline 15 & &,$\checkmark$ & & $\checkmark$ \\
\hline
\end{tabular}

Tabelle 6.3.: Messmatrix Potentialzug, Scheibe, $u_{\infty}=40 \mathrm{~m} / \mathrm{s}$.

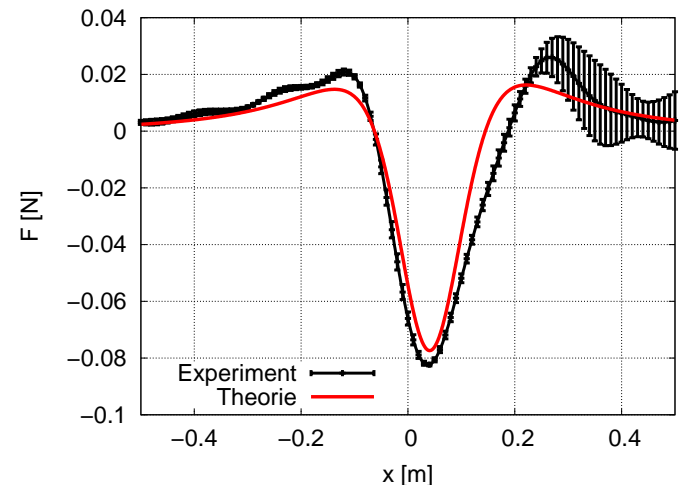

(a) x-Richtung

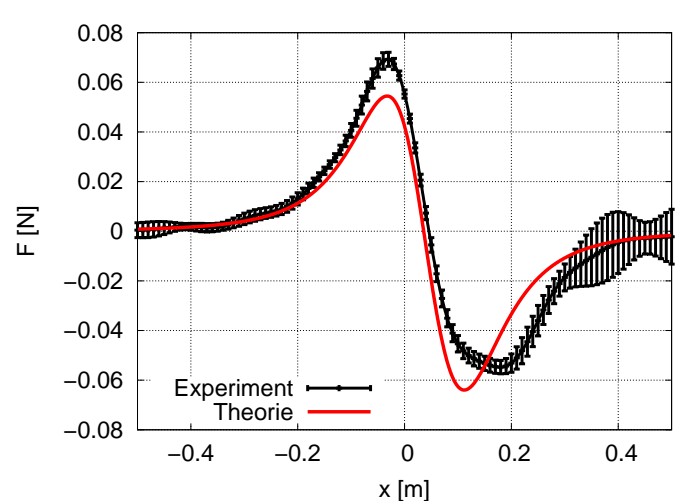

(b) y-Richtung

Abbildung 6.10.: Gemittelte Kraft mit RMS-Wert über fünf Fahrten bei der Vorbeifahrt des Potentialzugs auf die Styroporkugel $(R=2 \mathrm{~cm})$, bei einem Gleisabstand von $y_{M}=12 \mathrm{~cm}$ und einer Fahrtgeschwindigkeit von $u_{\infty}=40 \mathrm{~m} / \mathrm{s}$ verglichen mit der Potentialtheorie.

\subsubsection{Ergebnisse}

\section{Vergleich der Potentialtheorie mit den experimentellen Ergebnissen für die Kräfte auf die Kugel}

Abbildung 6.10 zeigt die über fünf Fahrten gemittelte Kraft der Kopfwelle auf die Styroporkugel $(R=2 \mathrm{~cm})$, bei einem Gleisabstand von $y_{M}=12 \mathrm{~cm}$ und einer Fahrtgeschwindigkeit von $u_{\infty}=40 \mathrm{~m} / \mathrm{s}$, verglichen mit der Potentialtheorie.

Die experimentellen Ergebnisse sind in guter Übereinstimmung mit der Theorie. Der relative Fehler zwischen Theorie und Experiment wird mithilfe von Formel 6.1 berechnet und beträgt $\vartheta_{x}=0,27$ in x-Richtung und $\vartheta_{y}=0,27$ in y-Richtung. Das ist eine gute Übereinstimmung zwischen Theorie und Experiment, im Vergleich zu den Messungen mit dem ICE3-Modell, bei denen der relative Fehler $\vartheta \approx 0,4$ beträgt.

Bei den Kräften in x-Richtung ist ersichtlich, dass beide Kurven sehr gut zusammenpassen, zumindest für den ersten Teil der Kraftkurve. Allerdings geschieht der zweite Anstieg der Kurve im Experiment signifikant später als in der Theorie. Von daher ist die 


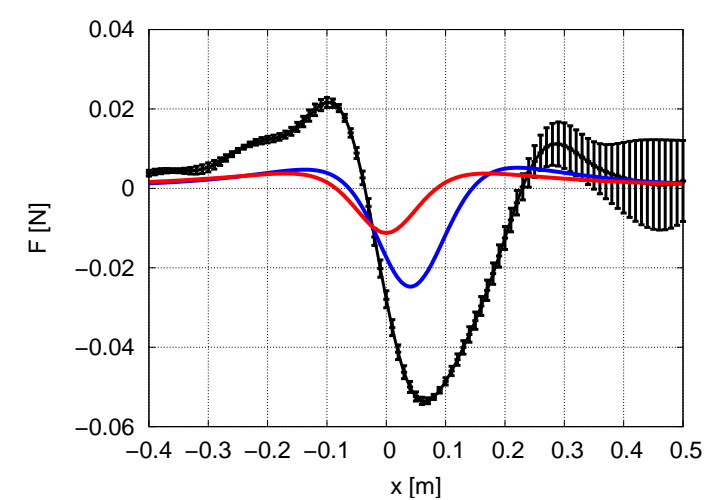

(a) x-Richtung

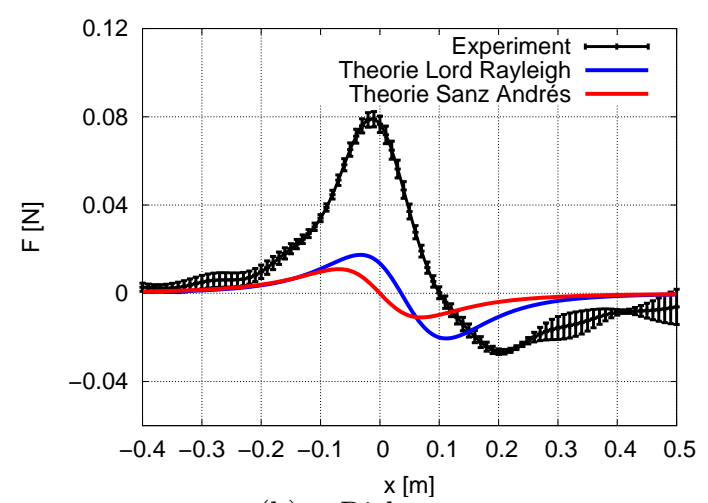

(b) y-Richtung

Abbildung 6.11.: Gemittelte Kraft über fünf Fahrten mit RMS-Wert (schwarze Linie) bei der Vorbeifahrt des Potentialzugs auf die Karbonscheibe $(R=2 \mathrm{~cm})$, bei einem Gleisabstand von $y_{M}=12 \mathrm{~cm}$ und einer Fahrtgeschwindigkeit von $u_{\infty}=40 \mathrm{~m} / \mathrm{s}$ verglichen mit der Potentialtheorie für Kreisscheiben (blaue Linie) und rechteckige Platten (rote Linie).

Phase der theoretisch vorhergesagten Fluktuation kürzer als die in den experimentellen Ergebnissen. Ebenso bei den Kräften quer zu Fahrtrichtung. Die gemessenen Kräfte auf die Kugel sind nicht symmetrisch, wie von der Theorie vorhergesagt. Im zweiten Teil der Kraftkurve, also in dem Bereich, in dem das Objekt an den Zug heran gezogen wird, dauert der Abklingvorgang der Kraft im Experiment länger als von der Theorie vorhergesagt. Das heißt, dass im Experiment das Objekt länger an den Zug „angesaugt“ wird, als von der Theorie vorhergesagt. Des weiteren stimmt die Kraftamplitude nicht exakt überein. Im ersten Teil der Kraftkurve wird die Kraft durch die Theorie unterschätzt und im zweiten Teil überschätzt. Der Kraftbetrag $\Delta F=F_{\max }-F_{\min }$ wird dadurch aber wieder korrekt eingeschätzt.

Die Unterschiede können durch die Vorderachse des Modells ausgelöst sein, da diese im theoretischen Modell nicht vorhanden ist. Die Strömung kann an den Rädern ablösen und dadurch können sich Wirbel entwickeln. Dennoch zeigt die generell gute Übereinstimmung zwischen Theorie und Experiment, dass die Interaktion zwischen Zug und der Kugel gut durch die Potentialtheorie beschrieben werden kann, wie bereits mit den Geschwindigkeitsmessungen in Abschnitt 4.5 gezeigt wurde. Allerdings wird noch zu untersuchen sein, ob diese beiden Abweichungen von der Potentialtheorie physikalische oder messtechnische Ursachen haben. 


\section{Vergleich der Potentialtheorie mit den experimentellen Ergebnissen für die Kräfte auf die Scheibe}

Abbildung 6.11 zeigt die gemittelte Kraft der Kopfwelle über fünf Fahrten bei der Vorbeifahrt des Potentialzugs auf die Scheibe $(R=2 \mathrm{~cm})$ bei einem Gleisabstand von $y_{M}=12 \mathrm{~cm}$ und einer Fahrtgeschwindigkeit von $u_{\infty}=40 \mathrm{~m} / \mathrm{s}$, verglichen mit der Potentialtheorie nach Lord Rayleigh [26] und der Theorie für eine rechteckige Platte nach Sanz-Andrés [29] (siehe Abschnitt 2.3.3 für die theoretischen Herleitungen).

Der Variationskoeffizient zwischen Rayleighs Theorie und dem Experiment beträgt $\vartheta_{x}=0,72$ in x-Richtung und $\vartheta_{y}=0,85$ in y-Richtung. Offensichtlich werden die gemessenen Lasten auf die Scheibe von der Theorie stark unterschätzt. Auch die vereinfachte Theorie für rechteckige Platten ist offensichtlich nicht geeignet, um die Kraft auf eine Kreisscheibe adäquat vorherzusagen. Beide theoretischen Ansätze unterschätzen sowohl die Kraftamplitude, als auch die Phase der Kraftfluktuation. Die potentialtheoretischen Modelle sind offensichtlich nicht geeignet, um Kräfte auf einer Scheibe vorherzusagen. Die Strömung löst vermutlich an den Ecken der Scheibe ab, so dass die Annahme einer reibungsfreien potentialtheoretischen Strömung für dieses Problem unzureichend ist. Es scheint, dass nichtlineare, insbesondere Reibungseffekte eine stärkere Rolle bei der Scheibe als bei der Kugel spielen. Zum Vergleich mit der stationären Aerodynamik sind in Abbildung 6.12 die theoretischen stationären Kräfte quer zur Fahrtrichtung für eine Kreisscheibe mit dem $C_{W}$-Wert=1,1 für die drei verschiedenen Scheiben aufgetragen. Die stationäre Kraft wird dabei wie folgt berechnet:

$$
F_{\text {stationär }}=C_{W} \frac{\rho}{2} v^{2} \pi R^{2} .
$$

Der stationäre Anteil unterschätzt die gemessene Kraft auf die Scheibe ebenfalls deutlich. Eine einfache Addition der beiden Kraftanteile, stationär und instationär, ergibt ebenfalls nicht die gemessene Kraft. Lediglich die Kombination der beiden Kraftanteile mit empirisch ermittelten Vorfaktoren bilden die gemessene Kraft auf die Scheibe ab, dieses auch für alle drei Scheibengrößen.

$$
F_{\text {theo,empirisch }}=\frac{11}{3}\left(C_{W} \frac{\rho}{2} v^{2} \pi R^{2}\right)+\frac{8}{3}\left(\frac{8}{3} \pi R^{3} \nabla p\right) .
$$

Es reicht also nicht, die Kraft auf die Scheibe rein instationär zu betrachten, wie bei der Kraft auf die Kugel. Hier stößt die Potentialtheorie offensichtlich an ihre Grenzen. Aber auch die stationäre Aerodynamik reicht als alleinige Theorie für die Kraft auf die Scheibe nicht aus. Hier scheint ein Mischeffekt vorzuliegen, da die Kraft auf die Scheibe sowohl von $R^{2}$ als auch von $R^{3}$ abhängt.

\section{Radiusvariation}

Nach den potentialtheoretischen Annahmen sind sowohl die Kräfte für die Kugel, als auch die Kräfte für die Scheibe proportional zu $R^{3}$. Zur Überprüfung dieser Annahme 
6. Instationäre Kraftmessungen an der TSG
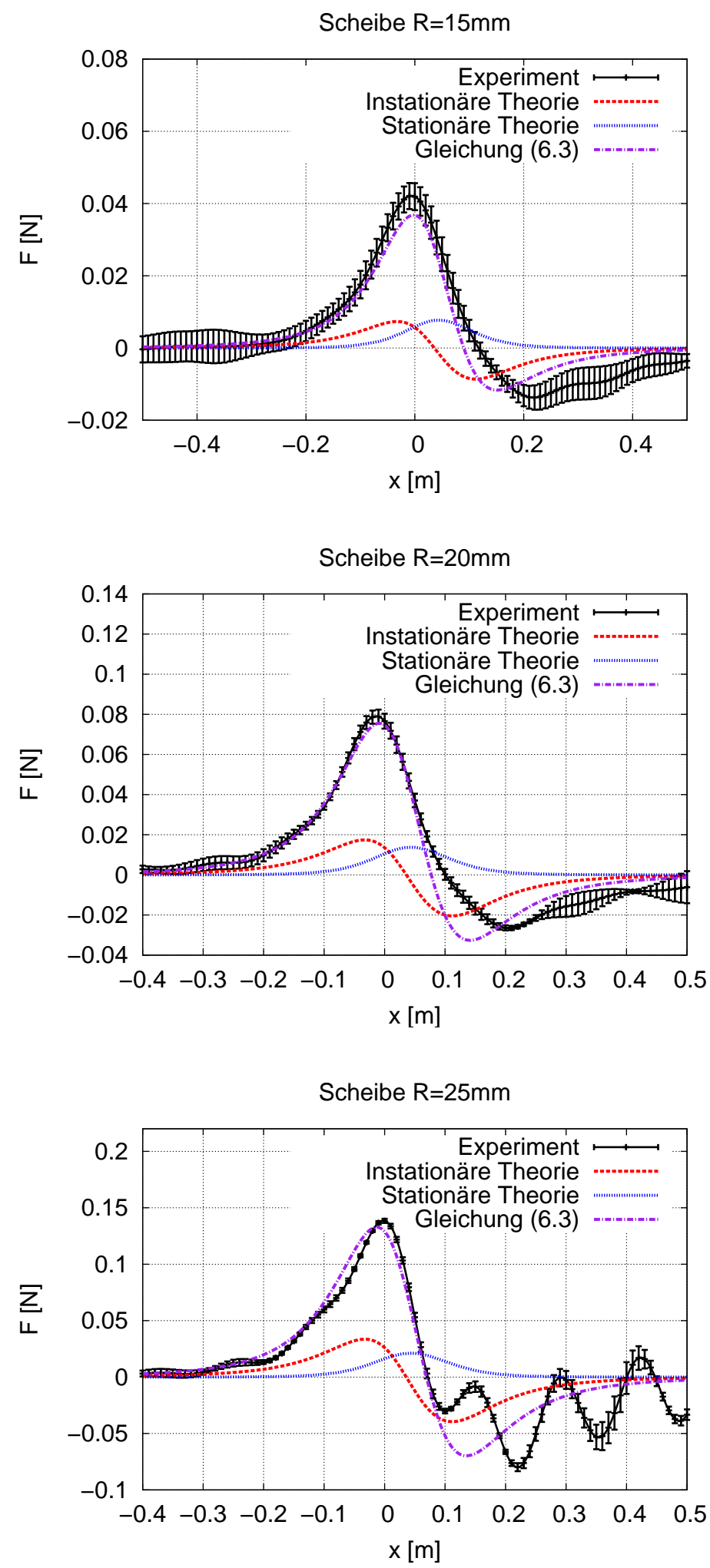

Abbildung 6.12.: Kraft quer zur Fahrtrichtung auf die Scheibe, verglichen mit stationärer 68 und instationärer Theorie. 


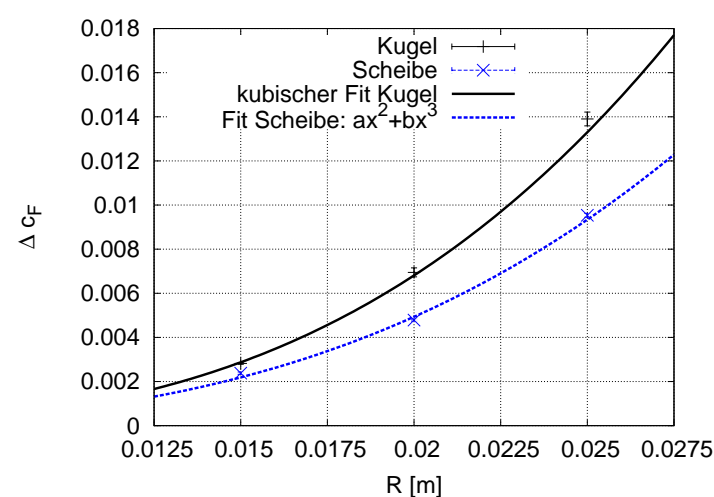

(a) x-Richtung

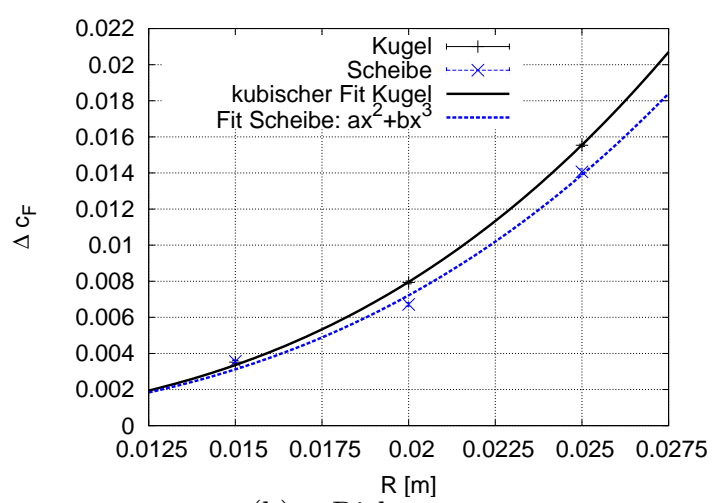

(b) y-Richtung

Abbildung 6.13.: Kräfte auf Styroporkugeln und Karbonscheiben unterschiedlicher Radien bei einem Gleisabstand von $y_{M}=12 \mathrm{~cm}$ und einer Fahrtgeschwindigkeitkeit von $u_{\infty}=40 \mathrm{~m} / \mathrm{s}$.

werden Styroporkugeln und Karbonscheiben verschiedener Größen verwendet (siehe Tabellen 6.2 und 6.3). In Abbildung 6.13 sind die Kraftbeträge $\Delta c_{F}=\Delta F /\left(0,5 \rho A_{\mathrm{Zug}} u_{\infty}^{2}\right)$ über die verwendeten Radien $R$ der Kugeln und der Scheiben aufgetragen und mithilfe von kubischen Funktionen approximiert worden.

Die kubische Approximation bestätigt die Annahme, dass die Kräfte auf die Kugel proportional zu $R^{3}$, also zum Kugelvolumen sind. Die Kräfte auf die Scheibe lassen sich zwar mit einer kubischen Funktion approximieren, allerdings sind drei Messpunkte für eine Approximation zu ungenau. Die Kraftamplituden bei der Scheibe sind kleiner als bei der Kugel. Die Amplitude der Kraft in x-Richtung ist um den Faktor 1,4 kleiner als bei der Kugel, wohingegen die Amplitude der Kraft in y-Richtung um den Faktor 1,1 kleiner ist als bei der Kugel. Das heißt, der Faktor variiert zwischen den Kräften in Xund y-Richtung. Theoretisch erwartet wurde hingegen der Faktor 2,3 (siehe Abschnitt 2.3.3, Gleichung 2.55).

Die Ergebnisse der kubischen Approximation lauten wie folgt:

$$
\begin{aligned}
\Delta c_{F_{x}}(\text { Kugel }) & =(851,2 \pm 15,9) \mathrm{m}^{-3} \cdot R^{3}, \\
\Delta c_{F_{y}}(\text { Kugel }) & =(995,4 \pm 6,5) \mathrm{m}^{-3} \cdot R^{3}, \\
\Delta c_{F_{x}}(\text { Scheibe }) & =(610,2 \pm 17,4) \mathrm{m}^{-3} \cdot R^{3} \\
\Delta c_{F_{y}}(\text { Scheibe }) & =(611,2 \pm 13,2) \mathrm{m}^{-3} \cdot R^{3}
\end{aligned}
$$

Die höheren Kräfte auf die Scheibe werden vermutlich durch Strömungsablösung an den scharfen Kanten der Scheibe verursacht. Allerdings würde eine stetige voll abgelöste Strömung um die Scheibe Kräfte, die proportional zu $R^{2}$ sind, verursachen. Da aber 


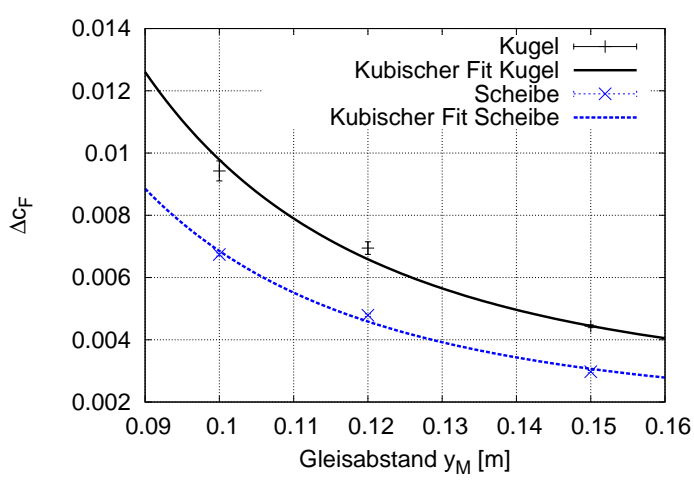

(a) x-Richtung

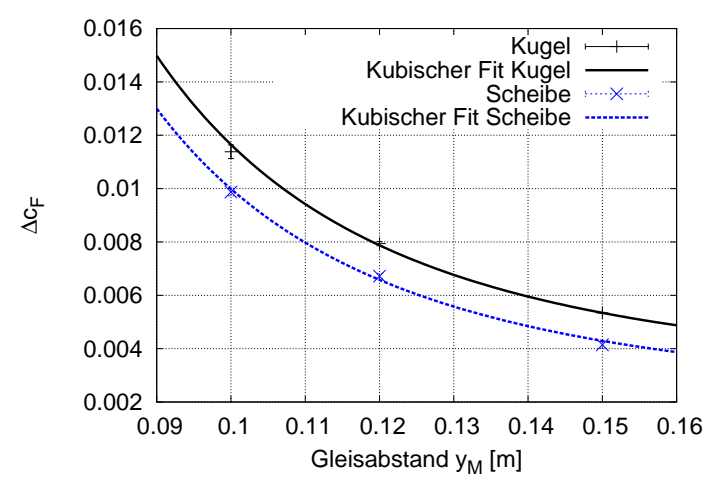

(b) y-Richtung

Abbildung 6.14.: Kräfte auf eine Kugel bzw. Scheibe $(R=2 \mathrm{~cm})$ bei verschiedenen Gleisabständen und einer Fahrtgeschwindigkeit von $u_{\infty}=40 \mathrm{~m} / \mathrm{s}$.

auch eine $R^{3}$-Proportionalität vorhanden ist, kann dies ein Hinweis darauf sein, dass die Instationarität ebenso eine Rolle spielt, obwohl Reibungseffekte involviert sind.

\section{Abstandsvariation}

Abbildung 6.14 zeigt den normierten Kraftbeiwert $\Delta c_{F}=\Delta F /\left(0,5 \rho A_{\mathrm{Zug}} u_{\infty}^{2}\right)$ aufgetragen über dem Gleisabstand $y_{M}$ für die Kugel und für die Scheibe. Ebenso wie bei den Versuchen mit dem ICE3-Modell (vgl. Abschnitt 6.1.2) ist sowohl für die Kugel als auch für die Scheibe die Kraft proportional zum inversen kubischen Gleisabstand. Die Approximation passt hier besser als bei dem ICE3-Modell, auch für kleine Gleisabstände, da das Potentialmodell nicht so einen großen Wagenkasten wie das ICE3-Modell hat und sich deshalb nicht so leicht Verwirbelungen aufgrund von Ablösungen an den scharfen Kanten des Radkastens oder der Räder bilden können.

Die Ergebnisse der kubischen Approximation der Form $\Delta c_{F}=a \cdot y_{M}^{-3}+b$ lauten wie folgt:

$$
\begin{aligned}
\Delta c_{F_{x}}(\text { Kugel }) & =(6,9 \pm 0,9) \cdot 10^{-6} \mathrm{~m}^{3} \cdot y_{M}^{-3}+(2,6 \pm 0,6) \cdot 10^{-3}, \\
\Delta c_{F_{y}}(\text { Kugel }) & =(8,5 \pm 0,3) \cdot 10^{-6} \mathrm{~m}^{3} \cdot y_{M}^{-3}+(2,9 \pm 0,2) \cdot 10^{-3}, \\
\Delta c_{F_{x}}(\text { Scheibe }) & =(5,3 \pm 0,5) \cdot 10^{-6} \mathrm{~m}^{3} \cdot y_{M}^{-3}+(1,5 \pm 0,3) \cdot 10^{-3}, \\
\Delta c_{F_{y}}(\text { Scheibe }) & =(8,1 \pm 0,5) \cdot 10^{-6} \mathrm{~m}^{3} \cdot y_{M}^{-3}+(1,9 \pm 0,3) \cdot 10^{-3}
\end{aligned}
$$

\section{Geschwindigkeitsvariation}

In Abbildung 6.15 ist der Kraftbetrag $\Delta F$ über die Fahrtgeschwindigkeit $u_{\infty}$ aufgetragen. Eine quadratische Approximation zeigt, dass die Kraft proportional zum Geschwindigkeitsquadrat ist. Nach Bernoulli ist dieses Ergebnis zu erwarten (siehe Abschnitt 2.3, 


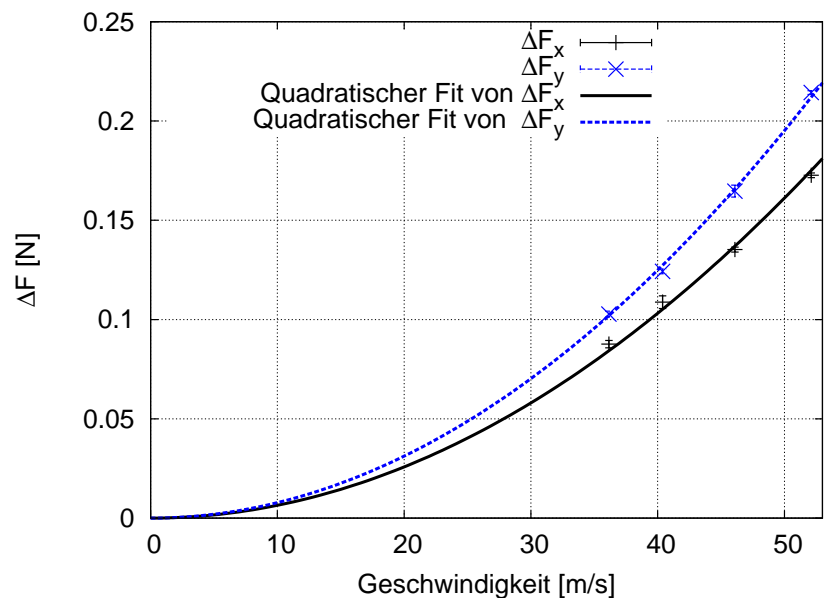

Abbildung 6.15.: Kraftamplitude bei der Kugel für verschiedene Zuggeschwindigkeiten $u_{\infty}$

Gleichung 2.26). Der Staudruck ist proportional zum Geschwindigkeitsquadrat, und da der Druck eine Kraft pro Flächenelement ist, ist ersichtlich, dass die Kraft ebenfalls proportional zum Geschwindigkeitsquadrat sein muss.

Die Ergebnisse der quadratischen Approximation lauten:

$$
\begin{aligned}
& \Delta F_{x}=(6,42 \pm 0,09) \cdot 10^{-5} \mathrm{~kg} \mathrm{~m} \cdot u_{\infty}^{2} \\
& \Delta F_{y}=(7,80 \pm 0,06) \cdot 10^{-5} \mathrm{~kg} \mathrm{~m} \cdot u_{\infty}^{2}
\end{aligned}
$$

\section{Höhenvariation}

Zusätzlich zum Abstand der Kugel zur Gleismitte wird die Höhe der Kugel über dem Boden variiert. Alle anderen Parameter werden nicht verändert. Der Gleisabstand beträgt $y_{M}=12 \mathrm{~cm}$, es wird eine Styroporkugel mit dem Radius $R=2 \mathrm{~cm}$ verwendet und die Fahrtgeschwindigkeit beträgt $u_{\infty}=40 \mathrm{~m} / \mathrm{s}$. Es werden folgende Höhen des Kugelmittelpunktes über der Schienenoberkante untersucht: $z_{M}=4,5 \mathrm{~cm}, z_{M}=6 \mathrm{~cm}, z_{M}=8 \mathrm{~cm}$ und $z_{M}=10 \mathrm{~cm}$.

In Abbildung 6.16 sind die gemessenen Kräfte auf die Kugel in x- und y-Richtung für verschiedene Höhen aufgetragen, im Vergleich mit der theoretischen Kurve für die Höhe $z_{M}=6 \mathrm{~cm}$. Die Abweichungen zwischen den gemessenen Kräften in x-Richtung und den theoretisch vorhergesagten Kräften sind sehr gering. Lediglich die Phase der Kraftfluktuationen ist bei allen Höhen länger als von der Theorie erwartet. Bei den Kräften in y-Richtung fällt auf, dass je höher sich die Kugel oberhalb des Bodens befindet, desto geringer die Abweichungen zwischen den gemessenen und den theoretischen Kräften werden. Die Phase $\Delta x$ zwischen dem Kraftmaximum und dem -minimum verringert sich bei größerer Höhe der Kugel. Bei $z_{M}=8 \mathrm{~cm}$ und $z_{M}=10 \mathrm{~cm}$ ist die Phase $\Delta x$ zwischen der theoretischen Kurve und den beiden experimentellen Kurven identisch. Dies erklärt 


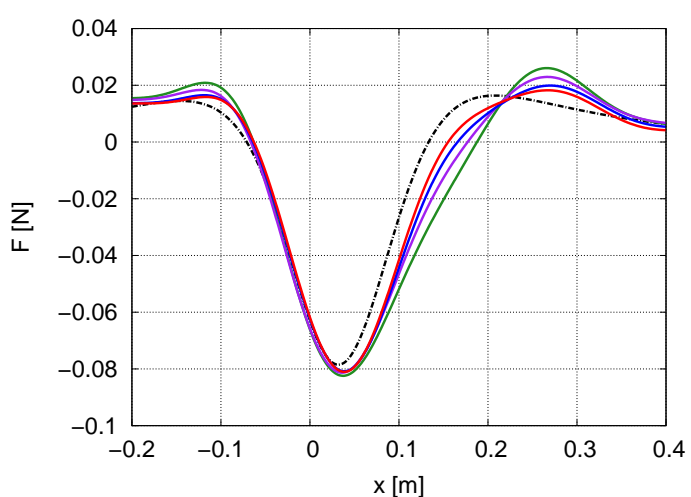

(a) x-Richtung

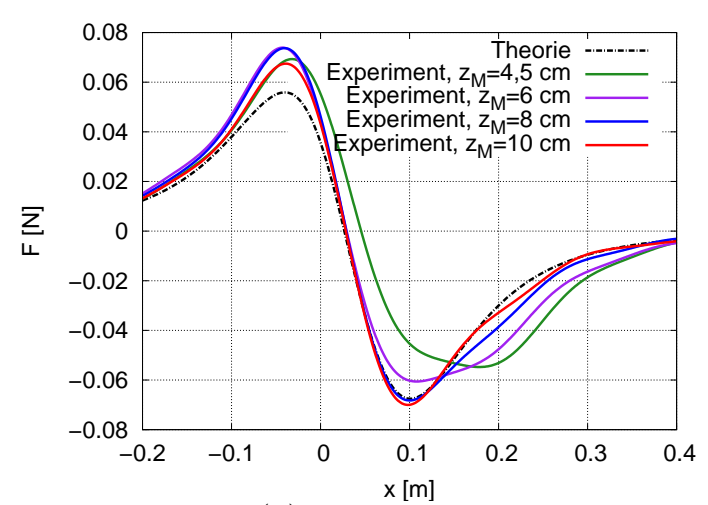

(b) y-Richtung

Abbildung 6.16.: Vergleich der experimentellen Kräfte auf die Kugel für verschiedene Höhen des Kugelmittelpunktes $(R=2 \mathrm{~cm})$ über der Schienenoberkante mit der potentialtheoretischen Kraftkurve für $z_{M}=6 \mathrm{~cm}$.

also die bereits im Abschnitt 6.3.2 gefundene Abweichung bezüglich der Phase. Bei einer niedrigen Höhe der Kugel über dem Boden, beeinflusst diese die Kraft auf die Kugel quer zur Fahrtrichtung erheblich. Dies wird in Abbildung 6.17 graphisch dargestellt. Dort ist die Phase $\Delta x$ über die Höhe der Kugel von der Unterkante der Kugel $\left(z_{M}-R\right)$ bis zur Schienenoberkante aufgetragen und mit einer invers kubischen Anpassung approximiert.

Dieser große Einfluss des Bodens auf die Kraft wird von der Potentialtheorie nicht vorhergesagt. Der Boden wird mithilfe der Spiegelebene zwar berücksichtigt, aber die Höhe der Kugel hat keinen signifikanten Einfluss auf die Kraft, weshalb in Abbildung 6.16 als Vergleich auch nur die theoretische Kraftkurve für eine Höhe aufgetragen wurde.

Die Wand des Potentialzugmodells verläuft nicht senkrecht zum Boden, sondern aufgrund der Quellverteilung im Zugkopf ist die Form konvex. Da für den Gleisabstand immer der Abstand von der Kugelmitte bis zur Gleismitte verwendet wird, ändert sich der reale Abstand zwischen Kugel und Zugwand, wenn sich die Höhe der Kugel verändert. Allerdings haben die Ergebnisse in Abschnitt 6.1.2 gezeigt, dass die Geometrie bei geringerem Gleisabstand einen größeren Einfluss auf die Kräfte gewinnt. Demzufolge müsste die Abweichung zwischen Theorie und Experiment größer werden, je höher die Kugel hängt, da sich zwischen $z_{M}=8 \mathrm{~cm}$ und $z_{M}=10 \mathrm{~cm}$ die breiteste Stelle des Zugs befindet. Genau das Gegenteil ist hier der Fall. Folglich scheint der Unterschied des Abstandes zwischen Kugel und Zugwand nicht verantwortlich für dieses Phänomen zu sein.

Ein möglicher Grund für die Abweichungen könnte sein, dass in der Nähe des Bodens Verwirbelungen entstehen, die von der Unterbodenströmung oder sich ablösender Grenzschicht des Zugmodells beziehungsweise Verwirbelungen durch Ablösung der Strömung 
6.3. Kraftmessungen mit einem Kraftmesselement: Potentialzug mit flachem Boden

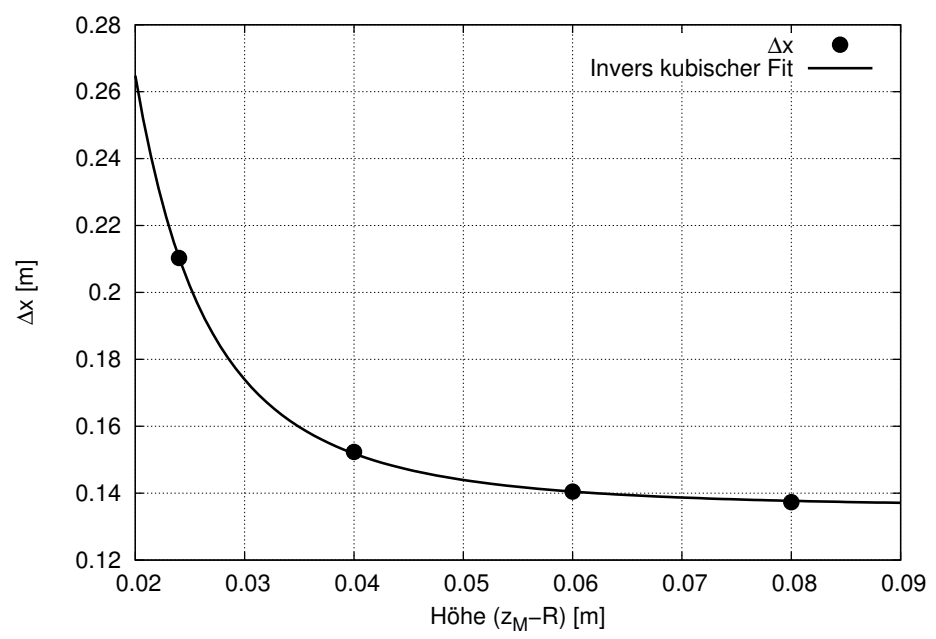

Abbildung 6.17.: Abhängigkeit der Phasenlänge $\Delta x$ von der Höhe der Kugel über der Schienenoberkante.

an den Rädern des Modells ausgelöst werden. Diese Fluktuationen scheinen die Kraft auf die Kugel in y-Richtung stark zu beeinflussen. Da in der Potentialtheorie reibungsfrei gerechnet wird, werden diese Phänomene in der Theorie nicht berücksichtigt.

\section{Verschiedene Zugköpfe}

Um den Einfluss der Zugkopfgeometrie zu bewerten, werden die Kräfte auf die Kugel $(R=2 \mathrm{~cm})$ bei drei verschiedenen Zuggeometrien gemessen. Es werden das ICE3-Modell, das Potentialzugmodell und das NGT Modell verwendet (siehe Abschnitt 3.2).

Das ICE3-Modell und der Potentialzug haben eine fast identische Querschnittfläche, wohingegen das NGT-Modell als ein Doppeldeckerzug eine größere Querschnittfläche hat. Daher werden die Kräfte bezüglich ihrer Querschnittfläche normiert, und die Kraftbeiwerte $c_{F}$ miteinander verglichen. Die Messungen werden bei einer Fahrtgeschwindigkeit von $u_{\infty}=40 \mathrm{~m} / \mathrm{s}$ und einem Gleisabstand von $y_{M}=12 \mathrm{~cm}$ durchgeführt. Pro Zugmodell werden fünf Fahrten durchgeführt und die gemessenen Kräfte anschließend gemittelt.

Abbildung 6.18 zeigt die Kraftbeiwerte $c_{F}$, induziert auf die Kugel durch die drei verschiedenen Zuggeometrien.

Während die Ergebnisse für das ICE3-Modell und den Potentialzug sehr ähnlich sind, weichen die Kräfte im Fall des NGT-Modells stark von diesen ab. Wie bereits in Abschnitt 6.1 gezeigt, werden die Kräfte des ICE3-Modells bereits gut durch das potentialtheoretische Modell beschrieben. Obwohl die Nasenlänge des ICE3-Modells fast doppelt so groß ist wie die des Potentialzugs, sind die Unterschiede bei den gemessenen Kräften auf die Kugel klein. Die Kraftamplitude $\Delta c_{F}$, die durch das NGT-Modell auf die Kugel induziert wird, ist hingegen kleiner als die Kraftamplituden bei den anderen beiden Zügen und die Phase der Kraftfluktuation durch das NGT-Modell ist länger. Dies ist auf die noch größere Nasenlänge des NGT-Modells verglichen mit den beiden anderen 


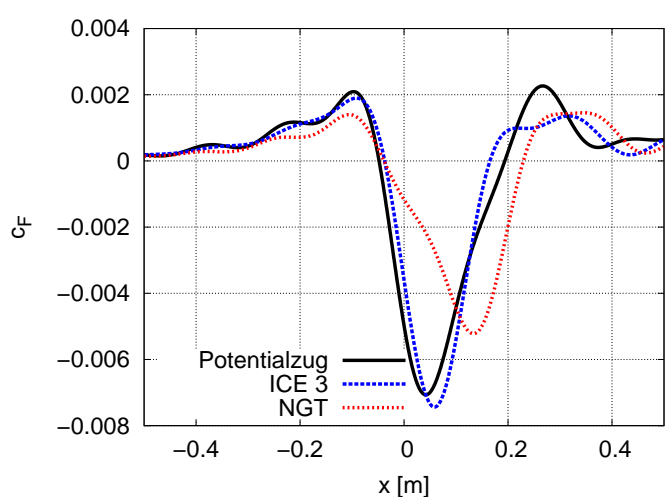

(a) x-Richtung

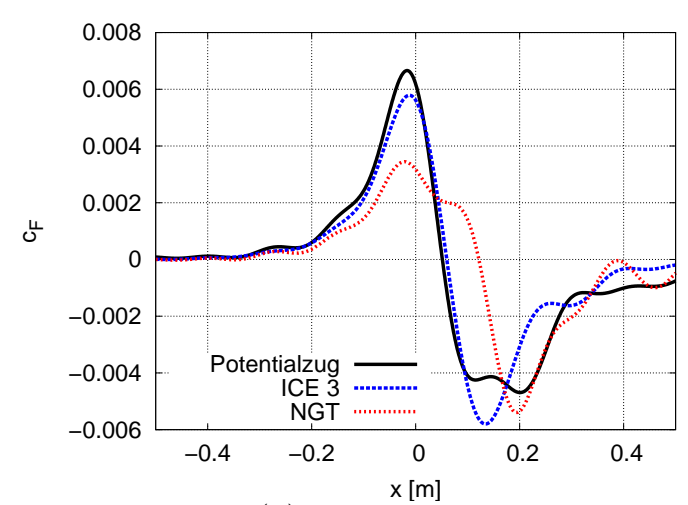

(b) y-Richtung

Abbildung 6.18.: Vergleich der induzierten Kraft auf eine Kugel $(R=2 \mathrm{~cm})$ durch verschiedene Zugköpfe bei einem Gleisabstand von $y_{M}=12 \mathrm{~cm}, z_{M}=$ $4,5 \mathrm{~cm}$ und einer Fahrtgeschwindigkeit von $u_{\infty}=40 \mathrm{~m} / \mathrm{s}$

Zügen zurückzuführen.

Allerdings zeigen die Ergebnisse, dass die Nasenlänge nicht der einzige ausschlaggebende Faktor für die Kräfte auf die Kugel sind, sondern auch die restliche Zuggeometrie eine essentielle Rolle für die Kopfwelle und die damit verbundenen Kräfte auf die Kugel spielt.

\subsection{Instationäre Kraftmessungen mit optischer Objektverfolgung}

Zusätzlich zu den instationären Kraftmessungen mit einem eindimensionalem Kraftmesselement werden instationäre Kraftmessungen mithilfe von optischer Objektverfolgung durchgeführt. Diese haben den Vorteil, dass sie nichtinvasiv sind, die Kugel also nicht befestigt werden muss, abgesehen von einer Aufhängung an einen langen Faden. Des Weiteren ist es möglich, die Bewegung in alle drei Raumrichtungen gleichzeitig zu verfolgen. Hier wird aber, genau wie bei der Waage, nur die Kraft in x- und y-Richtung, also in Fahrtrichtung und lateral zur Fahrtrichtung, betrachtet. Die Resonanzfrequenz $\omega_{0}=\sqrt{g / l}$ mit $\omega_{0}=2 \pi f_{0}$ beträgt $f_{0} \approx 0,7 \mathrm{~Hz}$ bei einer Fadenlänge von $l \approx 0,5 \mathrm{~m}$, wohingegen die Resonanzfrequenz des Masse-Feder-System der $R=25 \mathrm{~mm}$ Kugel mit der Kraftwaage und der Karbonhalterung $f_{\text {balance }} \approx 1300 \mathrm{~Hz}$ beträgt. Dies ist ein Vorteil gegenüber der Kraftwaage, da hier die Eigenfrequenz des Systems nicht mit berücksichtigt werden muss. Die im Folgenden beschriebene Messung ist eine Machbarkeitsstudie, ob die optische Objektverfolgung eine bessere Alternative zur Kraftmessung gegenüber den Messungen mittels Kraftmesselementen darstellen könnte. 


\subsubsection{Versuchsaufbau und Durchführung}

Die Versuche zur instationären Kraftmessungen mittels optischer Objektverfolgung werden ebenfalls an der TSG durchgeführt. Für die Versuche wird das 1:25 Potentialzugmodell und der flache Boden verwendet. Als Objekt wird eine Styroporkugel $(R=25 \mathrm{~mm})$, welche für den besseren Kontrast zum Hintergrund mit schwarzer Acrylfarbe versehen wurde, verwendet. Die Phantom v1210 Hochgeschwindigkeitskamera mit einem UV NIKKOR $105 \mathrm{~mm}$ 1:4,5 C-Mount Objektiv ist $135 \mathrm{~cm}$ oberhalb der Messstrecke an X95 Profilen befestigt. Dort ist auch die Kugel an einem dünnen Faden aufgehängt. Der Winkel zwischen Kugel und Kamera beträgt 10, $9^{\circ}$ und wird in der Auswertung heraus gerechnet. Der Gleisabstand zwischen Kugelmittelpunkt und Gleismitte beträgt $y_{M}=12 \mathrm{~cm}$. Die Höhe beträgt $z_{M}=4,5 \mathrm{~cm}$ von der Kugelmitte zur Schienenoberkante. Die Aufnahmefrequenz der Kamera beträgt $f=5000 \mathrm{~Hz}$. Es werden drei Fahrten durchgeführt. Die gemittelte Fahrtgeschwindigkeit beträgt $u_{\infty}=(39,8 \pm 0,2) \mathrm{m} / \mathrm{s}$. Der schematische Versuchsaufbau befindet sich in Abbildung 6.19.

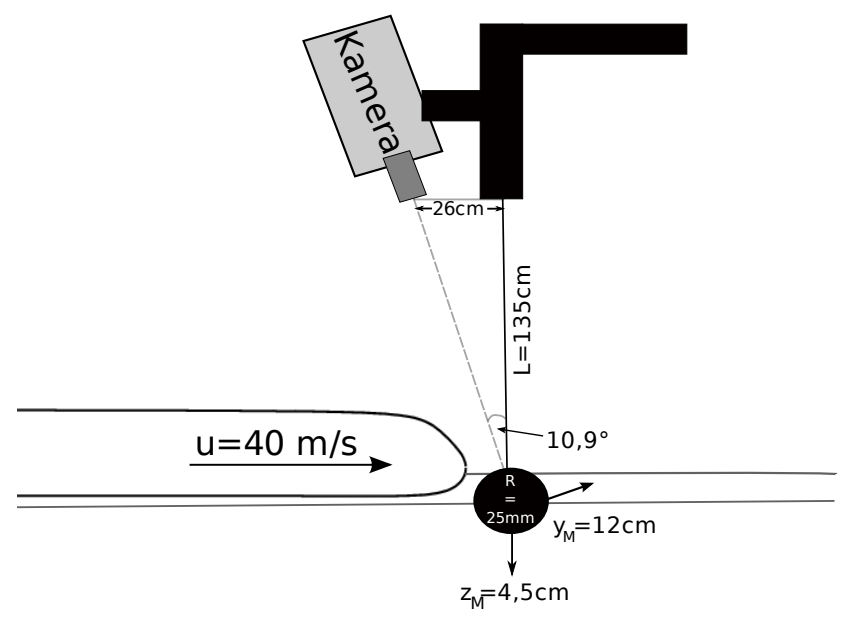

Abbildung 6.19.: Schematischer Versuchsaufbau zur instationären Kraftmessung mithilfe von optischer Objektverfolgung in der TSG.

\subsubsection{Ergebnisse}

Für die Auswertung wird eine Skriptsprache verwendet. In diesem Programm werden die Anfangsparameter, also die Position des Kugelmittelpunktes auf dem ersten Bild und der Radius der Kugel in Pixeln, definiert und die Verfolgungsroutine aufgerufen. Um die Position der Kugel bestimmen zu können, wird zunächst nur der Film eingelesen und als Einzelbilder wieder ausgegeben. Im Anschluss wird mit der Kugelposition, welche händisch aus dem ersten Bild mithilfe eines Bildbearbeitungsprogramm gefunden wurde, die Verfolgungsroutine gestartet. Das Programm schreibt schließlich die gefundene 


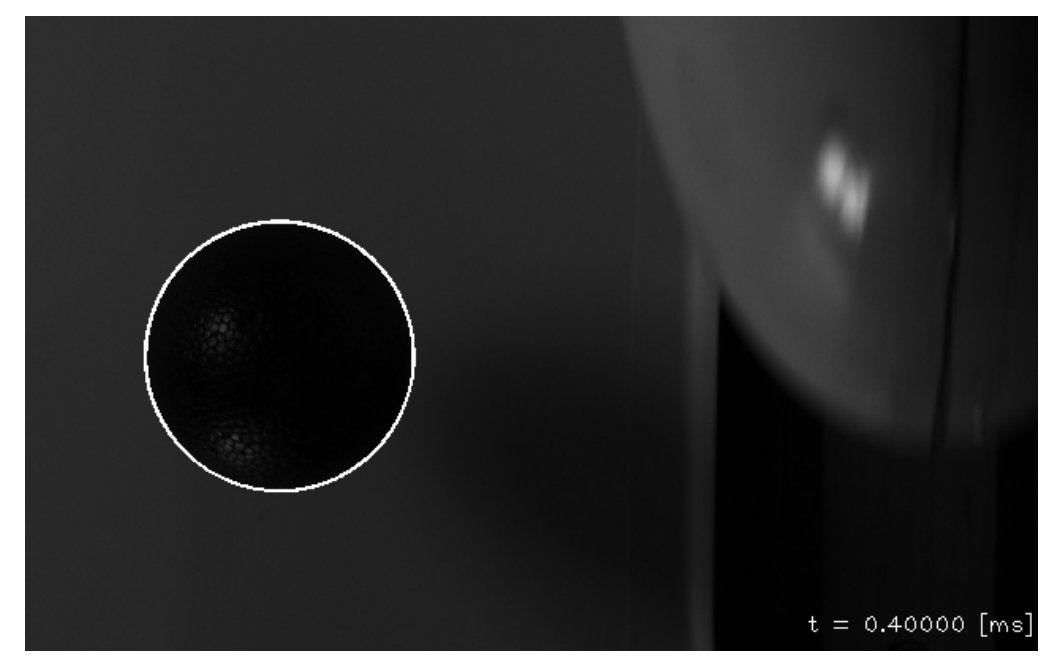

Abbildung 6.20.: Aufnahme der Vorbeifahrt des Potentialzuges an der Kugel $R=$ $25 \mathrm{~mm}$ zum Zeitpunkt $t=0,4 \mathrm{~ms}$. Die gefundenen Kantenpixel sind eingezeichnet.

Position der Kugel in x-,y- und z-Richtung, sowie den ermittelten Radius der Kugel in eine Textdatei. Aus dem Radius in Pixeln kann mit dem Radius in Millimetern die Bewegungstrajektorie von Pixeln wieder in SI-Einheiten umgerechnet werden. Bei diesem Versuchsaufbau ermittelt die Software für die $R=25 \mathrm{~mm}$ Kugel folgenden Radius in Pixeln:

$$
R=(84,8 \pm 0,1) \mathrm{px} .
$$

Das bedeutet, dass die Kantendetektionsroutine den Radius der Kugel und damit auch ihre Position mit einer Genauigkeit von $\sigma_{R}=0,15 \%$ ermittelt. Also entspricht

$$
\begin{aligned}
1 \mathrm{~mm} & \hat{=}(3,392 \pm 0,005) \mathrm{px} \\
\Rightarrow 1 \mathrm{px} & \hat{=}(294,8 \pm 0,5) \mu \mathrm{m},
\end{aligned}
$$

Dies ist die optische Auflösung. Abbildung 6.20 zeigt eine Aufnahme der Hochgeschwindigkeitskamera von der Vorbeifahrt des Potentialzuges an der Kugel zum Zeitpunkt $t=0,4 \mathrm{~ms}$. Der Zeitpunkt $t=0$ ist definiert als der Zeitpunkt, an dem sich Zugnase und Kugelmittelpunkt an derselben Stelle befinden. Die gefundene Objektkontur und die Trajektorie des Mittelpunktes bis zu diesem Zeitpunkt sind in der Aufnahme eingezeichnet.

Für die Berechnung der angreifenden Kraft muss der Weg zunächst zweimal abgeleitet werden. Da sich bei der Ableitung das Rauschen verstärkt, muss auf das Signal ein Filter angewendet werden. Zunächst wird auf das erhaltene Wegsignal ein Savitzky-Golay Filter [42] fünfter Ordnung mit 24 Datenpunkten angewendet, um das Signal zu glätten. Auf die erste und die zweite Ableitung wird dann jeweils ein Gleitender-Mittelwert-Filter [43] vierter Ordnung angewandt, der höhere Frequenzanteile aus dem Signal entfernt. Die 
Filter wurden so gewählt, da eine Variation der Datenpunkte und Ordnung bei dieser Konfiguration das beste Ergebnis ergeben, bei dem das Signal noch physikalisch ist, aber genügend Rauschanteile entfernt werden. Bei niedrigeren Ordnungen ist das Rauschen zu hoch und bei höheren Ordnungen wird das Signal verfälscht, da es unphysikalisch wird. Im Anschluss werden die daraus erhaltenen geglätteten Beschleunigungskurven der drei Versuche gemittelt und mit dem Kugelgewicht von $m_{B}=1,25 \mathrm{~g}$ multipliziert, um eine Kraft zu erhalten. Der Fehler der Kraftberechnung ergibt sich aus der Fehlerfortpflanzung der Fehler der Masse der Kugel $\sigma_{m}=0,01 \mathrm{~g}$, des Fehlers der Zeitaufnahme der Kamera, welcher $\sigma_{t}=1 \mathrm{~ns}$ beträgt. Der Fehler des Ortes entspricht dem Fehler der optischen Auflösung, also $\sigma_{x}=0,469 \mu \mathrm{m}$. Um die Fehlerfortpflanzung für die Kraft für all ihre unabhängigen Variablen durchführen zu können, wird die Kraft wie folgt ausgedrückt: Die Kraft ist gleich Masse mal Beschleunigung, also

$$
\begin{aligned}
F_{x} & =m \cdot a_{x} \\
\Rightarrow F_{x}(i) & =m \cdot \frac{\Delta u(i)}{\Delta t} \\
\Rightarrow F_{x}(i) & =m \cdot \frac{\Delta x(i)}{\Delta t^{2}} .
\end{aligned}
$$

Entsprechendes gilt für die Kraft in y-Richtung. Es ist $\Delta t=0,2 \mathrm{~ms}$, da die Aufnahmefrequenz $f=5000 \mathrm{~Hz}$ beträgt.

So ergibt sich für den Fehler $\sigma_{F_{x}}(i)$ der $\operatorname{Kraft} F_{x}(i)$ für jedes Bild folgende Formel $\left(\sigma_{F_{y}}(i)\right.$ entsprechend mit $\Delta y(i))$ :

$$
\begin{aligned}
\sigma_{F_{x}}(i) & =\sqrt{\sigma_{m}^{2}\left(\frac{\partial F_{x}}{\partial m}\right)^{2}+\sigma_{x}^{2}(i)\left(\frac{\partial F_{x}}{\partial \Delta x(i)}\right)^{2}+\sigma_{t}^{2}\left(\frac{\partial F_{x}}{\partial \Delta t}\right)^{2}} \\
\Rightarrow \sigma_{F_{x}}(i) & =\sqrt{\sigma_{m}^{2}\left(\frac{\Delta x(i)}{\Delta t^{2}}\right)^{2}+\sigma_{x}^{2}(i)\left(\frac{m}{\Delta t^{2}}\right)^{2}+\sigma_{t}^{2}\left(-\frac{2 m \Delta x(i)}{\Delta t^{3}}\right)^{2}} .
\end{aligned}
$$

In Abbildung 6.21 sind die gemittelten Kraftbeiwerte für die Kraftmessungen mit der Kraftwaage und für die Messung mittels optischer Objektverfolgung im Vergleich mit der Potentialtheorie für die $\mathrm{x}$ - und die $\mathrm{y}$-Richtung mit ihren jeweiligen Fehlerbalken aufgetragen. Bei dem Fehler der Messung mit der Kraftwaage handelt es sich um den RMS-Wert aus den fünf gemittelten Fahrten.

Zur besseren Vergleichbarkeit der Abweichungen sind in Abbildung 6.22 die Kraftänderungen über den Weg, also die Ableitungen $d F / d x$ der beiden Messungen und der Potentialtheorie aufgetragen. Zunächst ist zu erkennen, dass, obwohl die Kugel nur wenige Millimeter ausgelenkt wird, der Algorithmus die Verfolgung der Kugel gut durchführt und aus der Trajektorie eine Kraft berechnet werden kann. Die Kraftkurven in x- und yRichtung entsprechen auch qualitativ den theoretisch erwartetem Verlauf. In Abbildung 6.22 ist zu erkennen, dass die Messung mit der optischen Objektverfolgung, gerade die Kraft in x-Richtung, besser mit der Potentialtheorie übereinstimmt als die Kraftmessung 


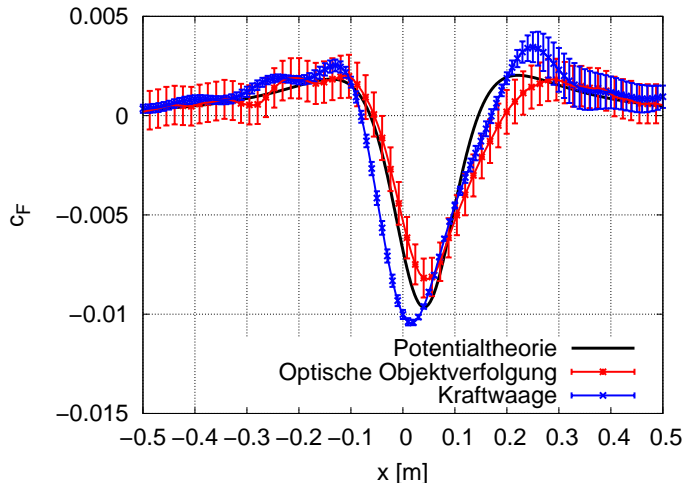

(a) x-Richtung

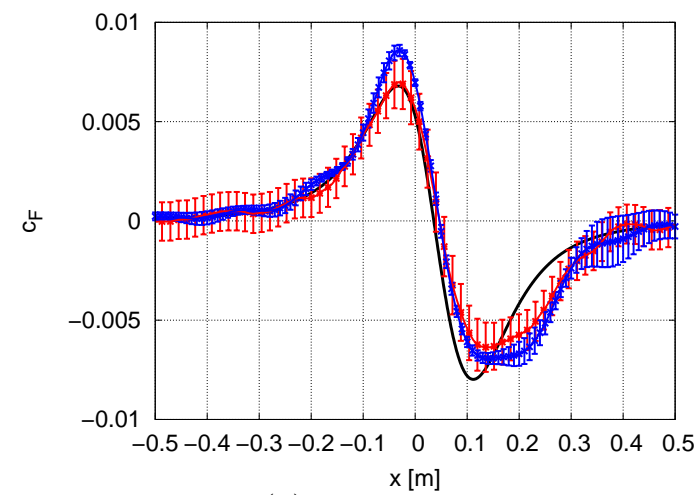

(b) y-Richtung

Abbildung 6.21.: Vergleich der Kraftbeiwerte auf eine Kugel $R=25 \mathrm{~mm}$, bei $y_{M}=$ $12 \mathrm{~cm}$ und $u_{\infty}=40 \mathrm{~m} / \mathrm{s}$ für verschiedene Messtechniken: Kraftwaage (blau) und optische Objektverfolgung (rot) im Vergleich mit der Potentialtheorie (schwarz).

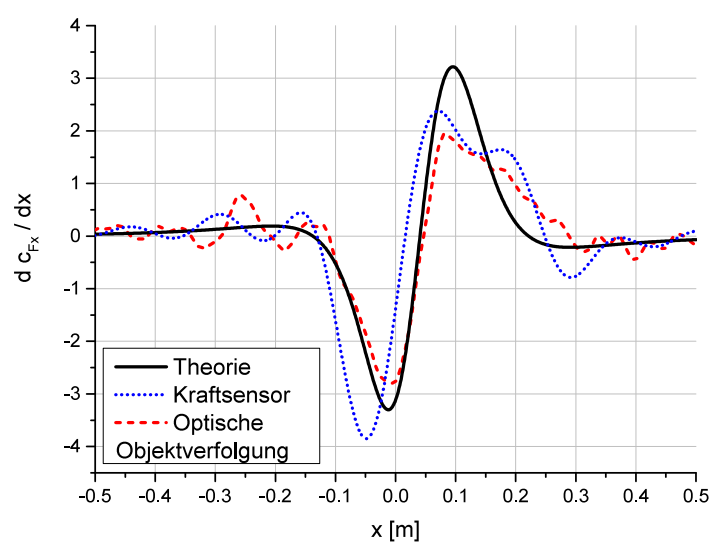

(a) x-Richtung

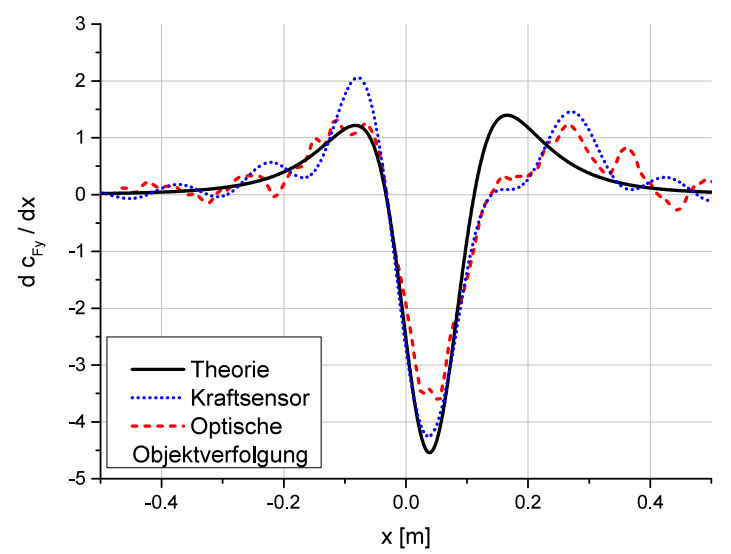

(b) y-Richtung

Abbildung 6.22.: Vergleich der Kraftänderung $\mathrm{d} c_{F} / \mathrm{d} x$ auf eine Kugel $R=25 \mathrm{~mm}$, bei $y_{M}=12 \mathrm{~cm}$ und $u_{\infty}=40 \mathrm{~m} / \mathrm{s}$ für verschiedene Messtechniken: Kraftwaage (blau) und optische Objektverfolgung (rot) im Vergleich mit der Potentialtheorie (schwarz). 
mit der Waage. Die Kraftamplitude $\Delta c_{F}$ (Abbildung 6.21) in x-Richtung ist $8 \%$ kleiner als von der Theorie vorhergesagt und damit auch kleiner als die gemessene Kraftamplitude $\Delta c_{F}$ mit der Kraftwaage, welche wiederum 12,5\% größer ist als die theoretische Kraftamplitude $\Delta c_{F}$.

In y-Richtung hingegen passt der Kraftverlauf der optischen Messung sehr gut mit der Theorie überein für den ersten Teil der Kraftkurve. Die Kraftamplitude $\Delta c_{F}$ der optischen Messtechnik ist 12\% kleiner als die theoretische Kraftamplitude, die Abweichung zwischen Waage und Theorie beträgt hier hingegen nur ca 4\%. Allerdings liegt die theoretische Kraftamplitude in beiden Fällen innerhalb der Fehlertoleranz der Messung mittels optischer Objektverfolgung. Im weiteren Verlauf der Kurve ist, sowohl beim Kraftbeiwert, als auch bei dessen Ableitung, dieselbe Abweichung bezüglich der Phase zu beobachten, wie bereits bei der Kraftwaage in Abschnitt 6.3. Diese Abweichung hat also keine messtechnische Ursache durch das Kraftmesselement, sondern es handelt sich offensichtlich um einen physikalischer Effekt, da dieser von verschiedenen Messtechniken messbar ist. In Abschnitt 6.3.2 wurde bereits gezeigt, dass diese Abweichung von der Höhe der Kugel über dem Boden abhängt und kleiner wird, je höher die Kugel hängt.

\subsection{Diskussion}

In diesem Kapitel wurden die Kraftmessungen mithilfe eines eindimensionalen Kraftmesselements vorgestellt und mit den potentialtheoretischen Überlegungen aus Kapitel 2 verglichen. Zunächst wurde eine Machbarkeitsstudie mit einem dreiteiligen ICE3Modell im Maßstab 1:25 durchgeführt. Diese haben gezeigt, dass es möglich ist, mithilfe des eindimensionalen Kraftmesselement die instationären Kräfte im Millinewton-Bereich adäquat zu messen. Bereits diese Messungen zeigen gute Übereinstimmung mit der Potentialtheorie, obwohl die Zuggeometrien nicht identisch sind.

Für die eigentliche Validierung der Potentialtheorie wurde der experimentelle Aufbau den theoretischen Vorgaben soweit wie möglich angepasst. Das Potentialmodell wurde im Maßstab 1:25 konstruiert und es wurde ein flacher Boden verwendet, welcher die Spiegelebene darstellt.

Bei diesen Experimenten zeigen sich besonders für die Kraft auf die Kugel gute Übereinstimmungen mit der Theorie. Die Abhängigkeit der Kraftamplitude vom kubischen Radius wurde experimentell sowohl für die Kugel als auch für die Scheibe als Objekt bestätigt. Allerdings ist die Kraftamplitude bei der Scheibe fast doppelt so groß wie von der Theorie vorhergesagt. Auch die Phase der Kraftfluktuation ist länger als von der Theorie vorhergesagt. Ein Vergleich mit der stationären Aerodynamik erklärt diese Abweichungen ebenfalls nicht. Bei einer voll abgelösten Strömung würde wiederum ein reiner $R^{2}$-Zusammenhang zu messen sein. Lediglich eine Kombination und Gewichtung der stationären und der instationären Kräfte gibt den experimentellen Verlauf wieder. 


\section{Instationäre Kraftmessungen an der TSG}

Für die Kugel hingegen ist die Übereinstimmung zwischen Theorie und Experiment sehr gut. Insbesondere quer zur Fahrtrichtung ist die Kraftamplitude größer als theoretisch vorhergesagt, vor allem beim Kraftmaximum treten Abweichungen um bis zu $20 \%$ auf. Beim Kraftminimum hingegen überschätzt die Theorie die wirkenden Kräfte zum Zug hin, so dass die Amplitude $\Delta F=F_{\max }-F_{\min }$ wieder gut mit der theoretischen Amplitude $\Delta F$ übereinstimmt. Es gibt ebenfalls, abhängig von der Höhe der Kugel über den Boden, Abweichungen in der Phase bei der Kraft in y-Richtung zwischen Theorie und Experiment. Je weiter in Richtung Boden sich die Kugel befindet, desto größer wird die Abweichung bezüglich der Phase. Dieser Zusammenhang findet sich aber nicht bei den Kräften in Fahrtrichtung. Möglicherweise entstehen durch die Räder oder die Unterbodenströmung des Zugmodells Verwirbelungen, welche näher am Boden einen größeren Einfluss auf die Kräfte haben als weiter oben.

Die Variation des Gleisabstandes zwischen Objekt und Gleismitte ergibt, sowohl bei den Messungen mit dem ICE3-Modell als auch mit dem Potentialmodell eine Abhängigkeit von $y_{M}^{-3}$ sowohl für die Kräfte auf die Kugel als auch für die Kräfte auf die Scheibe. Beim ICE3-Modell werden die Abweichungen zwischen Theorie und Experiment mit sinkendem Gleisabstand größer. Je näher sich das Objekt am Zug befindet, desto größer ist also der Einfluss der Zuggeometrie auf die Kräfte. Beim Potentialmodell ist dieser Unterschied geringer, da die Zuggeometrien in der Theorie und im Experiment, bis auf die Achsen und Räder, welche es in der Theorie nicht gibt, identisch sind.

Zwischen Geschwindigkeit und der Kraft auf die Kugel wurde ein quadratischer Zusammenhang ermittelt.

Die optische Kraftmesstechnik stellt eine gute Alternative zu den Kraftmessung mit dem Kraftmesselement dar. Die nichtinvasive Messung und die Möglichkeit die Kräfte in allen drei Bewegungsrichtungen gleichzeitig zu messen, ist ein großer Vorteil. Bei der Kraftwaage müssen für die gleichzeitige Messung der x- und y-Richtung zwei Elemente verwendet werden, welche unterschiedlich sein können, so dass hier eine höhere Fehlerquelle besteht. Allerdings kann die Kraftwaage die Kraft direkt messen, im Gegensatz zur optischen Objektverfolgung, welche die Trajektorie des Objekts ermittelt und daraus erst mittels zweifacher Ableitung die Kraft berechnet. Da die Auswertung der Kraftsignale mit der Kraftwaage viel schneller ist als mit der optischen Kraftmesstechnik und auch der experimentelle Aufbau leichter zu variieren ist, wurden die verschiedenen Messungen mit der Kraftwaage durchgeführt, um eine größere und bessere Statistik zu erhalten. Beide Messtechniken liefern aber ähnliche Ergebnisse und zeigen dieselbe Abweichung von der Theorie bezüglich der Phase, was auf einen strömungsphysikalische Ursache schließen lässt, die aber bereits durch die Höhe der Kugel über den Boden erklärt werden kann.

Dass die Amplitude der gemessenen Kraft mit dem Kraftmesselement generell höher ist als der theoretische Verlauf und der gemessene Verlauf mittels optischer Kraftmesstechnik, kann an der Waage selbst liegen: Sie kann bereits in ihren Resonanzbereich gelangt sein, da die Frequenz der Kraftkurve mit ca. $300 \mathrm{~Hz}$ nah an der Eigenfrequenz der Waage von ca. $1300 \mathrm{~Hz}$ liegt. Dennoch ist die theoretische Kurve noch innerhalb der 
Fehlertoleranz der optischen Messung, daher ist die Übereinstimmung zwischen Messung und Theorie, bis auf die Abweichung in der Phase, als sehr gut zu beurteilen. Der große Fehlerbereich kommt dadurch zustande, dass die optische Auflösung relativ gering ist. Die Kantendetektion wird besser, je mehr Pixel auf der zu detektierenden Kante liegen. Das lässt sich am einfachsten realisieren, in dem die Kugel den Großteil des Bildes einnimmt, so dass die Bewegung über möglichst viele Pixel erfolgt.

Die gefundenen Abweichungen bei den Kraftmessungen hängen jedoch nicht direkt von der induzierten Geschwindigkeit des Zuges auf das Objekt ab. In Kapitel 4 wurde gezeigt, dass das Strömungsfeld um den Zug als potentialtheoretisch angesehen werden kann. Wie bereits in Abschnitt 2.3.1 theoretisch hergeleitet wurde, beeinflussen primär instationäre Effekte die Kraft auf die Kugel. Die Geschwindigkeit selbst spielt nur eine untergeordnete Rolle bei der Kraft auf die Kugel. Es kann daher ausgeschlossen werden, dass die vom Zug induzierte Strömung die Abweichung der Kraft von der potentialtheoretisch vorhergesagten Kraft verursacht. 

KAPITEL 7

Kräfte auf Objekte in periodischen instationären Strömungen

\subsection{Theoretische Überlegungen - Erweiterung des potentialtheoretischen Modells}

In den vorangegangen Kapiteln wurde mithilfe von zwei verschiedenen Kraftmesstechniken gezeigt, dass die Kräfte auf Objekte in instationären Strömungen, wie sie bei der Zugvorbeifahrt auftreten, gut mithilfe der Potentialtheorie vorhergesagt werden können. Geschwindigkeitsmessungen mithilfe von Hitzdrahtanemometrie haben ebenfalls gezeigt, dass die Kopfwelle des vorbeifahrenden Zuges als potentialtheoretische Strömung angenommen werden kann. Des Weiteren wurde gezeigt, dass diese Kräfte von instationären Effekten dominiert werden, da sie proportional zum Volumen der Kugel sind und nicht zu ihrer Querschnittfläche, wie es in der stationären Aerodynamik der Fall ist.

Dennoch sind sowohl bei der Messung mit der Kraftwaage als auch bei der Messung mithilfe von optischer Objektverfolgung Abweichungen in der Kraftamplitude bezogen auf die Potentialtheorie aufgetreten. Dies deutet darauf hin, dass zusätzliche physikalische Effekte eine Rolle spielen und die Potentialtheorie erweitert werden muss, um eine genauere Vorhersage der Kräfte treffen zu können. Für die Scheibe existieren bisher keine weiteren analytischen Lösungen, daher beschränken sich die weiteren Untersuchungen auf Kugeln als einfache geometrische Körper.

Für die Kugel wurde in Kapitel 2.3.2 gezeigt, dass die Situation der Zugvorbeifahrt vereinfacht dargestellt werden kann, indem eine Kugel, an der eine ebene Welle gestreut wird, betrachtet wird. Die Kraft $F_{\text {tot }}$ auf die Kugel setzt sich aus der Kraft der einfallenden Welle $F_{\text {ein }}$ auf die Kugel und der Kraft der gestreuten Welle $F_{\text {ges }}$ zusammen:

$$
F_{\text {tot }}=F_{\text {ein }}+F_{\text {ges }} .
$$


Nach Gleichung 2.40 gilt:

$$
F_{\text {tot }}=F_{\text {ein }}+\frac{1}{2} F_{\text {ein }}
$$

Also:

$$
F_{\text {tot }}=\frac{4}{3} \pi R^{3} \rho \frac{\partial u}{\partial t}+\frac{1}{2} \cdot \frac{4}{3} \pi R^{3} \rho \frac{\partial u}{\partial t}=2 \pi R^{3} \rho \frac{\partial u}{\partial t} .
$$

Dieser Kraftterm 7.3 ist für die Beschreibung der Kraft auf Kugeln in instationärer Strömung allein nicht ausreichend. Er berücksichtigt beispielsweise nicht die Reibung, da er keinen Term für die Viskosität enthält. Gleichung 7.3 ist gültig für reibungs- und drehungsfreie Strömungen, also für Potentialströmungen. Da das Experiment leichte Abweichungen zu dieser theoretischen Gleichung aufweist, sind noch weitere Terme für die vollständige Beschreibung der Kraft erforderlich, die auch die Reibung enthalten und somit die reine Potentialtheorie erweitern.

Im Jahr 1851 untersuchte Stokes erstmalig die Kräfte einer einfach gradlinig harmonisch oszillierenden Kugel, eines Zylinders und einer unendlich langen Platte [3]. Er vernachlässigte die nichtlinearen Terme der Navier-Stokes-Gleichung und gab eine exakte Lösung für den instationären Fall an.

Später untersuchten Basset [4], Boussinesq [5] und Oseen [6] die geradlinige Bewegung einer Kugel mit einer großen Beschleunigung in einem viskosen Fluid. Sie vernachlässigten ebenfalls die nichtlinearen Terme der Navier-Stokes-Gleichung und stellten fest, dass die Kraft einer beschleunigten Kugel nicht nur von ihrer unmittelbaren Geschwindigkeit und Beschleunigung abhängt, sondern zusätzlichen von einem integralen Ausdruck, der die gesamte Geschichte der Beschleunigung mit berücksichtigt. Die Gleichung von Basset, Boussinesq und Oseen (BBO) ist nur gültig für langsam bewegte, aber schnell beschleunigte Kugeln und lautet wie folgt [11]:

$$
-F=\underbrace{6 \pi R \mu v}_{F_{D}}+\underbrace{\frac{1}{2} \cdot\left(\frac{4}{3} \pi R^{3}\right) \rho a}_{F_{A}}+\underbrace{6 R^{2}(\pi \mu \rho)^{\frac{1}{2}} \int_{0}^{t} \frac{a\left(t^{\prime}\right)}{\sqrt{\left(t-t^{\prime}\right)}} d t^{\prime}}_{F_{H}} .
$$

Dabei handelt es sich bei $\mu$ um die dynamische Viskosität des Fluids, $v$ ist die Geschwindigkeit und $a=\partial u / \partial t$ die Beschleunigung des Fluids. Bei $R$ handelt es sich um den Kugelradius.

Der erste Term der Gleichung wird als „Reibungskraft" (engl: drag force) bezeichnet und mit $F_{D}$ gekennzeichnet. Wenn ein Partikel sich durch ein Fluid bewegt, wird es aufgrund von Reibung abgebremst. Für $R e=0$ gilt die Stokes'sche Formel $\vec{F}_{D}=6 \pi \mu R \vec{u}$. Für alle größeren Reynoldszahlen muss $F_{D}$ experimentell bestimmt werden.

Beim zweiten Term handelt es sich um die „zusätzliche-Massekraft“ (englisch: added mass force). Dieser wird mit $F_{A}$ gekennzeichnet und beschreibt die zusätzliche Trägheit des Systems, da ein beschleunigter Körper das ihn umgebende Fluid mitbewegen muss. Der dritte und letzte Term wird als „Basset-Kraft“ (engl: history force) bezeichnet und 
mit $F_{H}$ gekennzeichnet. Sie beschreibt die Kraft, die aufgrund der verzögerten Bewegung der Grenzschicht entsteht. Da sie aufgrund des numerisch auszuwertenden Integrals schwierig zu implementieren ist [7], wird diese oft vernachlässigt, allerdings kann sie bei großen Beschleunigungen eine Rolle spielen [10].

Offensichtlich entspricht die zusätzliche-Masse-Kraft $F_{A}$ genau dem potentialtheoretischen Kraftterm $F_{\text {ges }}$, welcher durch die gestreute Welle an der Kugel erzeugt wird (Gleichung 7.3). Bei der Kraft auf die Kugel in instationären Strömungen, wie zum Beispiel der Zugvorbeifahrt, könnten also die anderen beiden Terme, insbesondere der Basset-Term, eine Rolle spielen.

Die Kräfte auf beschleunigte Kugeln für kleine Reynoldszahlen wurden bisher hauptsächlich numerisch untersucht [7-10]. Odar und Hamilton untersuchten das Problem hingegen experimentell [11]. Sie modifizierten die BBO-Gleichung, in dem sie die einzelnen Terme mit konstanten Faktoren multiplizierten, die sie schließlich experimentell bestimmten. So lautet die modifizierte BBO-Gleichung nach Odar und Hamilton wie folgt [11]:

$$
-F=\frac{1}{2} C_{D} \pi R^{2}|v| v+C_{A}\left(\frac{4}{3} \pi R^{3}\right) \rho a+C_{H} R^{2}(\pi \mu \rho)^{\frac{1}{2}} \int_{0}^{t} \frac{a\left(t^{\prime}\right)}{\sqrt{\left(t-t^{\prime}\right)}} d t^{\prime} .
$$

Den Reibungskoeffizienten $C_{D}$ entnehmen sie aus [44]. $C_{A}$ und $C_{H}$ müssen experimentell bestimmt werden, wenn jedoch die Geschwindigkeit klein gegenüber der Beschleunigung ist, gelte der potentialtheoretische Wert $C_{A}=0,5[11]$.

Des Weiteren führen $\mathrm{OH}$ einen dimensionslosen Paramter ein, den sie „Beschleunigungszahl" (englisch: acceleration number) nennen und der das Verhältnis zwischen konvektiver und lokaler Beschleunigung darstellt [11]:

$$
A c=\frac{v^{2}}{a D},
$$

mit $D$ als Durchmesser der Kugel. Wenn die konvektive Beschleunigung klein gegenüber der lokalen Beschleunigung ist, ist die Beschleunigungszahl klein. Eine kleine Beschleunigungszahl lässt sich erreichen, wenn die Geschwindigkeit der Kugel klein ist, ihre Beschleunigung und Durchmesser hingegen groß [11].

\subsection{Experimentelle Untersuchungen}

Zur Bestimmung von $C_{A}$ und $C_{H}$ führten Odar und Hamilton ein Experiment durch, bei welchem eine Kugel mit $D=2,5$ Zoll $=6,35 \mathrm{~cm}$ in einem Öltank mithilfe eines Schwungrades hin und her bewegt wird [11]. So ergeben sich die folgenden Bewegungsgleichungen für die Kugel:

$$
\begin{aligned}
& x(t)=A_{0} \cos (\omega t) \\
& v(t)=-A_{0} \omega \sin (\omega t) \\
& a(t)=-A_{0} \omega^{2} \cos (\omega t) .
\end{aligned}
$$


Eingesetzt in 7.5 ergibt sich [11]:

$$
\begin{aligned}
-F(t) & =\frac{1}{2} C_{D} \pi R^{2} A_{0}^{2} \omega^{2}|\sin (\omega t)| \sin (\omega t)+C_{A}\left(\frac{4}{3} \pi R^{3}\right) \rho A_{0} \omega^{2} \cos (\omega t) \\
& +C_{H} R^{2}(\pi \mu \rho)^{\frac{1}{2}} A_{0} \omega^{3 / 2}(\sin (\omega t)+\cos (\omega t)) .
\end{aligned}
$$

Diese Überlegungen gelten bisher nur für kleine Reynoldszahlen. OH benutzen in ihrem Experiment Reynoldszahlen bis $R e=62$. Auch in den numerischen Berechnungen in [7-10] werden nur Reynoldszahlen bis maximal $R e=50$ untersucht.

\subsubsection{Versuchsanordnung}

Bei der Zugvorbeifahrt treten allerdings größere Reynoldszahlen bei der Kugel auf. Mit $u=5 \mathrm{~m} / \mathrm{s}$ und $R=25 \mathrm{~mm}$ ergibt sich eine Reynoldszahl von $R e=17544$. Da in der TSG nur begrenzt verschiedene Geschwindigkeiten gefahren werden können, wird ein neues Experiment realisiert, bei dem sich die Kugel im Geschwindigkeitsfeld eines großen Lautsprechers befindet. Dabei können verschiedene Frequenzen verwendet werden und größere Reynoldszahlen realisiert werden. Die Kugel befindet dabei sich in einem gleichmäßig oszillierendem, instationären Strömungsfeld. Der Unterschied zum Experiment von Odar und Hamilton ist, dass sich in diesem Fall die Luft bewegt, und nicht die Kugel aktiv in einem ruhenden Fluid bewegt wird. Hier muss die einfallende Welle aus Gleichung 7.3 berücksichtigt werden. Im potentialtheoretischen Fall gilt:

$$
\begin{aligned}
F_{A}^{*} & =F_{\text {tot }} \\
\Rightarrow F_{A}^{*} & =\frac{4}{3} \pi R^{3} \rho \frac{\partial u}{\partial t}+\frac{1}{2} \cdot \frac{4}{3} \pi R^{3} \rho \frac{\partial u}{\partial t} \\
\Rightarrow F_{A}^{*} & =2 \pi R^{3} \rho \frac{\partial u}{\partial t}
\end{aligned}
$$

Analog zu Odar und Hamilton [11] wird der Koeffizient $C_{A}^{*}$ eingeführt, so dass im potentialtheoretischen Fall $C_{A}^{*}=2$ gilt.

$$
F_{A}^{*}=C_{A}^{*} \pi R^{3} \rho \frac{\partial u}{\partial t} .
$$

Entsprechend gilt für die Kraft auf die Kugel in instationärer Strömung mit ebener Anströmung folgende Formel:

$$
-F(t)=\frac{1}{2} C_{D} \pi R^{2}|v| v+C_{A}^{*}\left(\pi R^{3}\right) \rho \frac{\partial u}{\partial t}+C_{H} R^{2}(\pi \mu \rho)^{\frac{1}{2}} \int_{0}^{t} \frac{a\left(t^{\prime}\right)}{\sqrt{\left(t-t^{\prime}\right)}} d t^{\prime} .
$$

Es wird untersucht, ob sich die Ergebnisse von Odar und Hamilton [11] auch für größere Reynoldszahlen reproduzieren lassen. Dies wurde in der Literatur bisher noch nicht untersucht. Des Weiteren wird untersucht, ob es Frequenzen gibt, bei denen die BassetKraft relevant wird, oder ob sich der Faktor $C_{A}^{*}$ der zusätzliche-Masse-Kraft verändert 
und wovon dieser abhängt. Die daraus gewonnen Ergebnisse dienen der Optimierung der Kraftvorhersage bei der instationären Strömungssituation der Zugvorbeifahrt.

Für die Experimente wird ein Lautsprecher mit einem akustischen Niederfrequenzwandler verwendet [45]. Der Lautsprecher deckt ein Frequenzspektrum von $f=30 \mathrm{~Hz}$ bis $f=1 \mathrm{kHz}$ ab. Die Lautsprechermembran hat einen Durchmesser von $d=460 \mathrm{~mm}$. Der Lautsprecher wird in eine Holzbox mit einem Volumen von 6001 eingebaut und an einen Verstärker angeschlossen, welcher wiederum an einem Frequenzgenerator angeschlossen ist. Mit dem Frequenzgenerator werden Sinusschwingungen verschiedener Frequenzen auf den Verstärker und damit den Lautsprecher gegeben, sodass die Bewegungsgleichungen 7.7-7.9 auch in diesem Fall angewendet werden können. Die Kraftgleichung lautet schließlich:

$$
\begin{aligned}
-F(t) & =\frac{1}{2} C_{D} \pi R^{2} A_{0}^{2} \omega^{2}|\sin (\omega t)| \sin (\omega t)+C_{A}^{*}\left(\pi R^{3}\right) \rho A_{0} \omega \cos (\omega t) \\
& +C_{H} R^{2}(\pi \mu \rho)^{\frac{1}{2}} A_{0} \omega^{3 / 2}(\sin (\omega t)+\cos (\omega t)) .
\end{aligned}
$$

Für die Beschleunigungszahl gilt dann entsprechend:

$$
\begin{aligned}
\hat{A} c & =\frac{\hat{v}^{2}}{\hat{a} \cdot 2 R}, \\
\Rightarrow \hat{A c} & =\frac{A_{0}^{2} \omega^{2}}{A_{0} \omega^{2} \cdot 2 R} \\
\Rightarrow \hat{A} c & =\frac{A_{0}}{2 R}
\end{aligned}
$$

Das bedeutet, dass die Beschleunigungszahl auch als das Verhältnis zwischen Hub und Durchmesser dargestellt werden kann. Bei einem großen Hub und einem kleinen Radius wird $A c$ entsprechend groß.

\subsection{Vermessung des Geschwindigkeitsfeldes des Lautsprechers}

Zunächst wird untersucht, ob das Geschwindigkeitsfeld des Lautsprechers gleichmäßig ist. Für die Geschwindigkeitsmessung, die zeitgleich mit der Kraftmessung mit einem Kraftmesselement durchgeführt wird (siehe Abschnitt 7.4), wird ein Laser-Doppler-Vibrometer verwendet $[46,47]$. Das Vibrometer misst die Auslenkung und die Geschwindigkeit der Lautsprechermembran. Unter der Annahme, dass sich die Luft oberhalb der Membran, wie eine Säule, exakt genauso wie die Membran bewegt, kann die Geschwindigkeit der Luft, also die Schallschnelle, an der Stelle der Kugel als die Membrangeschwindigkeit angenommen werden. Um zu überprüfen, ob das der Fall ist, wird das Feld zusätzlich dreidimensional mit MiCROFLOWN-Sonden vermessen [48].

Die Microflown-Sonden geben ein Spannungssignal aus. Um dieses in ein Geschwindigkeitssignal umzurechnen, wird das Spannungssignal einer Microflown-Sonde bei einer 


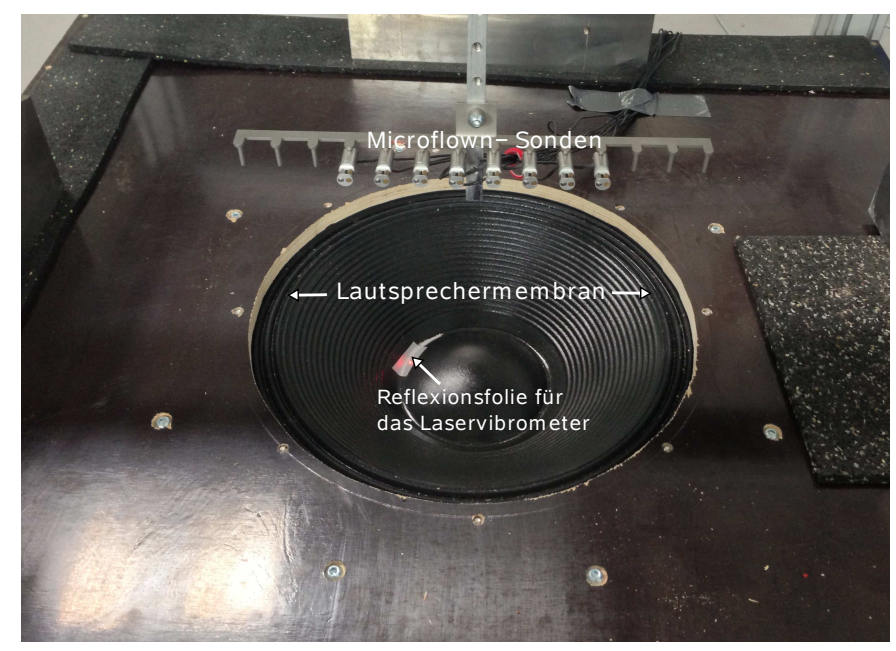

Abbildung 7.1.: Versuchsaufbau der Geschwindigkeitsmessungen mit MicroflownSonden.

Höhe von $h=2 \mathrm{~cm}$ über der Membranmitte gemessen. Mithilfe der Membrangeschwindigkeit, gemessen mit dem Laservibrometer, wird das Signal der Microflown-Sonde normiert. Das Laservibrometer ist so konfiguriert, dass $1[\mathrm{~V}]=1[\mathrm{~m} / \mathrm{s}]$ entspricht, die Geschwindigkeit lässt sich daher direkt ablesen.

$$
\begin{aligned}
\frac{U_{\text {Microflown }}[\mathrm{mV}]}{v_{\text {Laservibro }}[\mathrm{m} / \mathrm{s}]} & =\text { norm }\left[\frac{\mathrm{mV}}{\mathrm{m} / \mathrm{s}}\right] \\
\Rightarrow v_{\text {Microflown }}[\mathrm{m} / \mathrm{s}] & =\frac{U_{\text {Microflown }}[\mathrm{mV}]}{\text { norm }\left[\frac{\mathrm{mV}}{\mathrm{m} / \mathrm{s}}\right]}
\end{aligned}
$$

Diese Normierung wird auf alle acht Microflown-Sonden angewendet, welche in $x$ Richtung im Abstand von $2 \mathrm{~cm}$ zueinander angebracht sind. Für die Messung in $z$ Richtung befinden sich in der Halterung des Sondenhalters Löcher im Abstand von $3 \mathrm{~cm}$, in welche der Sondenhalter geschraubt werden kann. Abbildung 7.1 zeigt den Lautsprecher in der Box mit den 8 Microflown-Sonden im Halter oberhalb der Membran montiert.

In horizontaler Richtung ( $x$-Richtung) wird von der Mitte der Lautsprechermembran ausgehend $(x=0 \mathrm{~cm})$ in $\Delta x=2 \mathrm{~cm}$ bis $x= \pm 7 \mathrm{~cm}$ gemessen. In vertikaler Richtung wird in $\Delta z=3 \mathrm{~cm}$ Abständen von $z=-11 \mathrm{~cm}$ bis $z=15 \mathrm{~cm}$ gemessen. Die Höhe $z=0 \mathrm{~cm}$ entspricht der Kugelposition bei den späteren Kraftmessungen. Diese entspricht $h=12,5 \mathrm{~cm}$ gemessen von der Oberkante der Lautsprechermembran.

Abbildung 7.2 zeigt die Geschwindigkeitsverteilung in der $x$-z-Ebene beispielhaft für eine Frequenz $(f=30 \mathrm{~Hz})$. Es ist deutlich zu erkennen, dass das Feld nicht sehr gleichmäßig ist und die Geschwindigkeit vor allem in der Höhe stark abnimmt. Das bedeutet, dass die gemessene Membrangeschwindigkeit mithilfe des Laservibrometers 


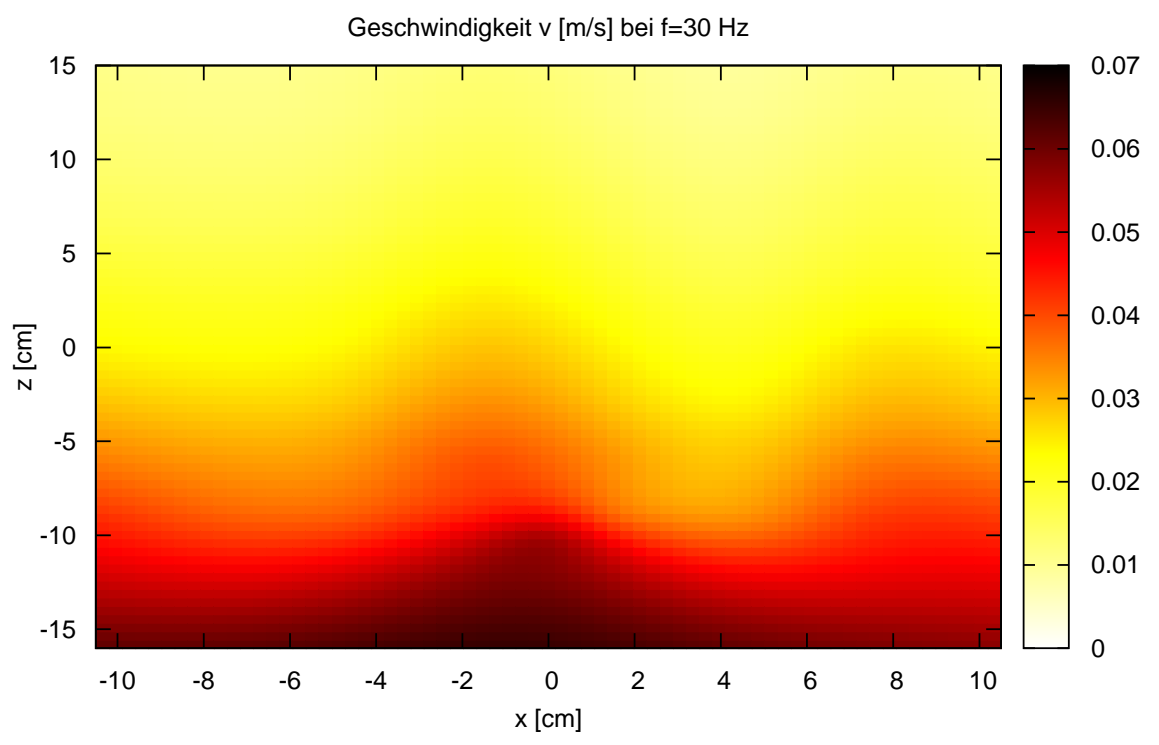

Abbildung 7.2.: Geschwindigkeitsfeld bei $f=30 \mathrm{~Hz}$.

nicht gleich der Geschwindigkeit der Luft an der Kugelposition ist.

In Abbildung 7.3 sind die gemessenen Geschwindigkeiten in Abhängigkeit von der Frequenz für verschiedene $z$-Positionen oberhalb der Membran aufgetragen. Die Position $z=0$ entspricht der Position der Kugel bei den Kraftmessungen. Das GeschwindigkeitsFrequenz-Spektrum ändert sich bei den unterschiedlichen $z$-Positionen nicht. Sie unterscheiden sich lediglich durch einen für jede Frequenz konstanten Faktor.

Um die Geschwindigkeit der Luft auf Kugelhöhe zu berechnen, wird daher das Verhältnis der Geschwindigkeit der Membran, gemessen mit dem Laservibrometer, zu der Geschwindigkeit an der Kugelposition, also der Schallschnelle, gemessen mithilfe der MicroflownSonden benötigt, da während der Kraftmessungen nur die Membrangeschwindigkeit mithilfe des Laservibrometers gleichzeitig gemessen werden kann. Die Microflown-Sonden und die Kugel würden sich ansonsten gegenseitig beeinflussen.

In Abbildung 7.4 sind die Frequenz-Geschwindigkeits-Spektren des Laservibrometers und einer Microflown-Sonde an der Kugelposition aufgetragen. Beide Kurven verlaufen qualitativ gleich, es unterscheidet sie lediglich ein frequenzspezifischer Faktor. Das bedeutet, dass das Laservibrometer weiterhin für die Geschwindigkeitsmessungen verwendet werden kann. Das Signal muss lediglich mit einem Korrekturfaktor multipliziert werden, um die Geschwindigkeit der Luft an der Kugelposition zu erhalten. 
7. Kräfte auf Objekte in periodischen instationären Strömungen

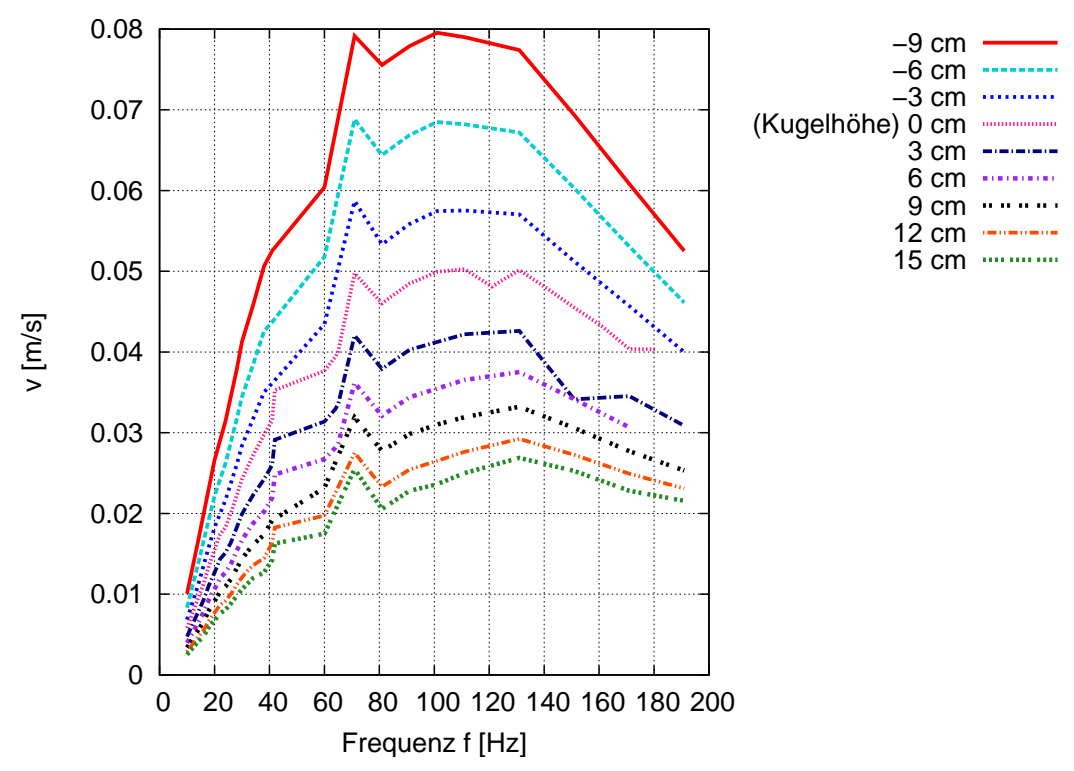

Abbildung 7.3.: Geschwindigkeits-Frequenz-Spektrum bei verschiedenen Höhen über der Membran, relativ zur Kugelposition, gemessen mit Miroflown-Sonden. $0 \mathrm{~cm}$ entspricht der Höhe der Kugel oberhalb der Membran.

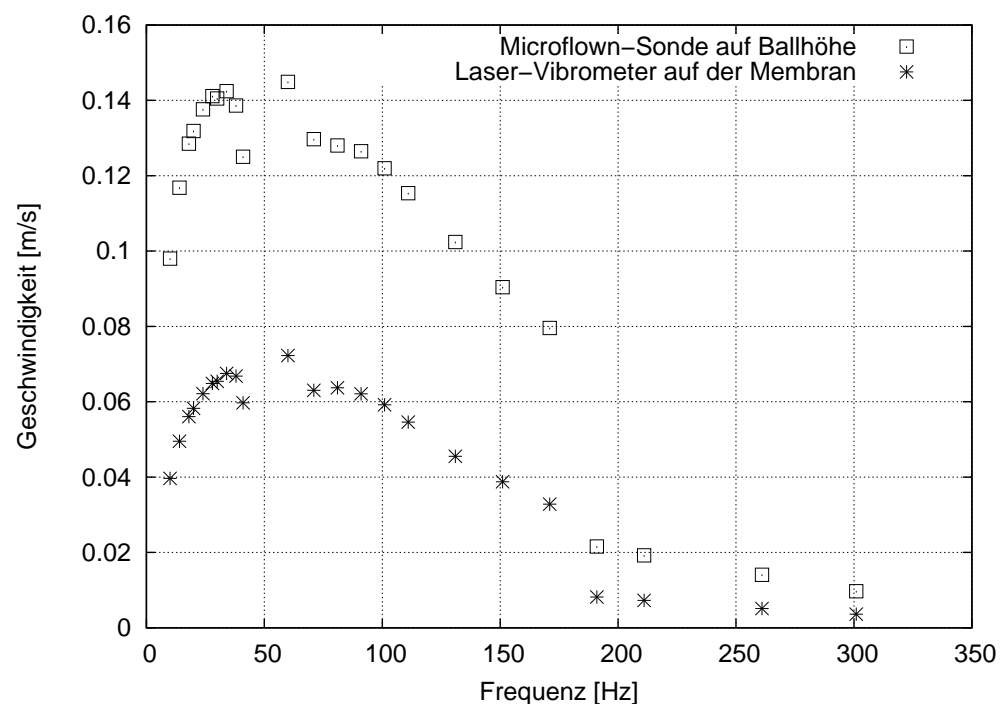

Abbildung 7.4.: Vergleich der Geschwindigkeiten an der Membran (Laservibrometer) und auf Kugelhöhe (Microflown-Sonden). 


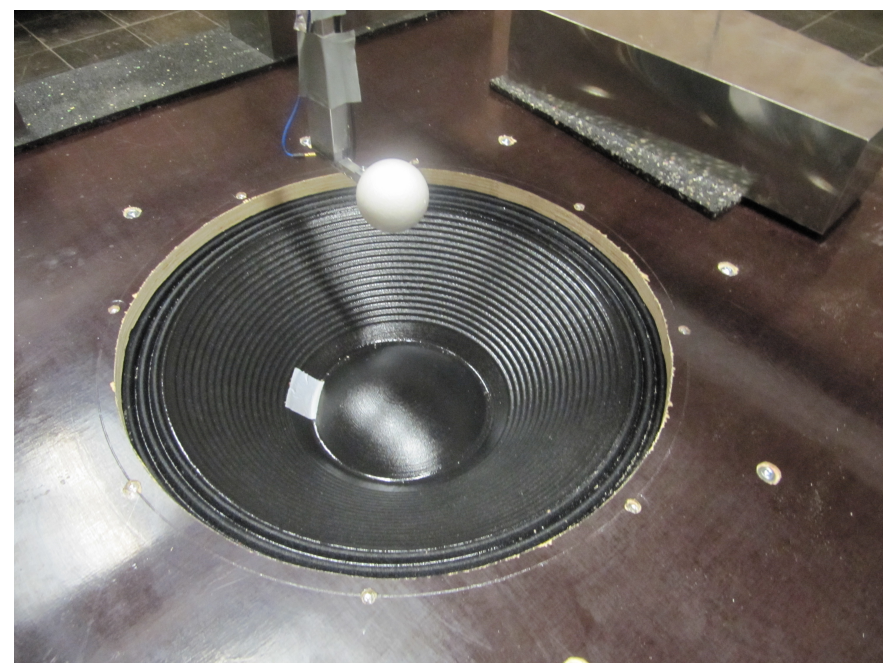

Abbildung 7.5.: Versuchsaufbau der Kraftmessungen im instationären Lautsprecherfeld mithilfe eines Kraftmesselements.

Der Umrechnungsfaktor ergibt sich wie folgt:

$$
\text { Faktor }(f)=\frac{v_{\text {Kugelhöhe }(\text { Microflown })}(f)}{v_{\text {Membran(Laservibrometer })}(f)} .
$$

In Tabelle C.1 in Anhang C sind die einzelnen Normierfaktoren für die Geschwindigkeit auf Kugelhöhe für jede gemessene Frequenz eingetragen. Diese werden für die Geschwindigkeitskorrektur des Laservibrometersignals verwendet. Diese Faktoren gelten jedoch nur für die hier spezifisch verwendete Kugelhöhe von $h=22,5 \mathrm{~cm}$. Die Geschwindigkeit der Luft auf Kugelhöhe wird dann wie folgt für jede gemessene Frequenz korrigiert:

$$
v_{\text {neu }}(f)=v_{\text {mess }}(f) \cdot \operatorname{Faktor}(f) .
$$

\subsection{Messung der Kraft auf eine Kugel mithilfe eines Kraftmesselements im instationären Feld eines Lautsprechers}

Es werden die Frequenzen $f=30 ; 71 ; 110 ; 131 \mathrm{~Hz}$ bei verschiedenen Amplituden untersucht, die am Frequenzgenerator eingestellt werden. Der Verstärker befindet sich auf maximaler Einstellung.

\subsubsection{Resonanzen der Kraftwaage}

Für die Messungen mithilfe des Kraftmesselementes ist es wichtig, dass die Resonanz des Masse-Feder-Systems, bestehend aus Kugel, Halterung und Waage, weit oberhalb der Frequenzen liegen, welche im Versuch verwendet werden. Um die Resonanzfrequenzen des 


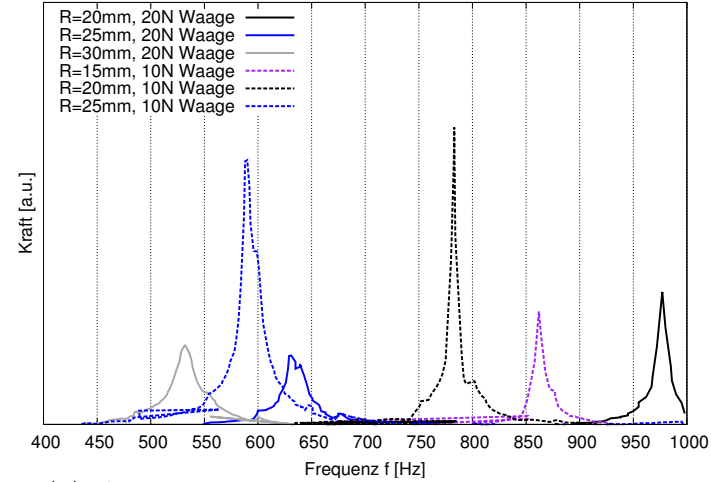

(a) Aluhalterung, $10 \mathrm{~N}$ Waage und $20 \mathrm{~N}$ Waage

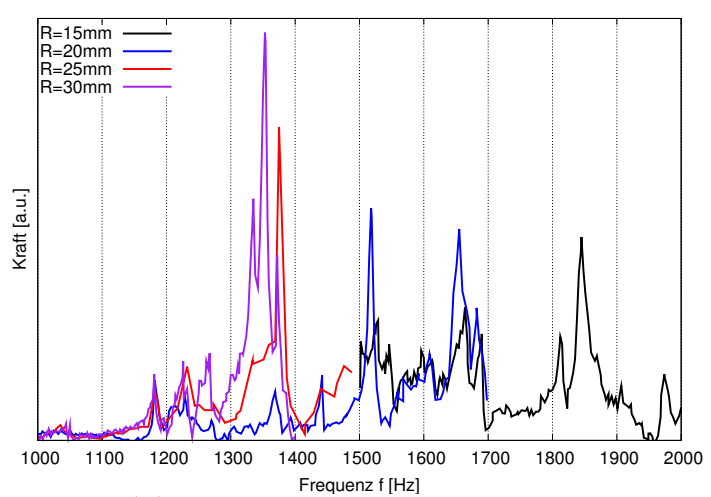

(b) Karbonhalterung, 20N Waage

Abbildung 7.6.: Resonanzen des Masse-Feder-Systems: Waage-Kugel mit unterschiedlichen Halterungen und Waagensteifigkeiten.

\begin{tabular}{c||c|c|c|c|}
\multirow{2}{*}{ Radius $[\mathrm{mm}]$} & \multicolumn{2}{c|}{ Kugel mit Aluhalterung } & \multicolumn{2}{c|}{ Kugel mit Karbonhalterung } \\
& Masse $[\mathrm{g}]$ & Resonanzfrequenz $[\mathrm{Hz}]$ & Masse $[\mathrm{g}]$ & Resonanzfrequenz $[\mathrm{Hz}]$ \\
\hline 15 & 4,23 & $862(10 \mathrm{~N}) / 1106(20 \mathrm{~N})$ & 1,00 & $1845(20 \mathrm{~N})$ \\
\hline 20 & 6,00 & $783(10 \mathrm{~N}) / 977(20 \mathrm{~N})$ & 1,50 & $1518(20 \mathrm{~N})$ \\
\hline 25 & 6,51 & $590(10 \mathrm{~N}) / 631(20 \mathrm{~N})$ & 2,50 & $1375(20 \mathrm{~N})$ \\
\hline 30 & 8,09 & $518(10 \mathrm{~N}) / 531(20 \mathrm{~N})$ & 3,00 & $1353(20 \mathrm{~N})$ \\
\hline
\end{tabular}

Tabelle 7.1.: Resonanzfrequenzen von Waage, Halterung und Kugel für verschiedene Kugelradien und Halterungen/Waage-Kombinationen.

Systems zu ermitteln, werden mithilfe des Frequenzgenerators Sinusschwingungen verschiedener Frequenzen von $f=100 \mathrm{~Hz}$ bis $f=2000 \mathrm{~Hz}$ auf den Lautsprecher gegeben. Die Kraftwaage mit der Kugel befindet sich auf einer Höhe von $h=22,5 \mathrm{~cm}$ oberhalb des tiefsten Punktes der Lautsprechermembran (vgl. Abbildung 7.5). Als Kraftmesselement wird ein $10 \mathrm{~N}$ und ein $20 \mathrm{~N}$-Element KD45 der Firma ME-Meßsysteme verwendet (vgl. Abb. 5.1). Es werden Kugeln mit den Radien $R=15 ; 20 ; 25 ; 30 \mathrm{~mm}$ verwendet. Alle Kugeln sind jeweils mit einer Aluminiumhalterung bzw. einer Karbonhalterung versehen.

Abbildung 7.6 zeigt die Signale der Waage bei verschiedenen Frequenzen. Die Maxima entsprechen den jeweiligen Resonanzfrequenzen. In Tabelle 7.1 sind die einzelnen Resonanzfrequenzen für jede Kugel/Halter/Waage-Kombination und die Masse der jeweiligen Kugel inklusive Halterung eingetragen.

Die Eigenfrequenzen der Kugel mit der Aluhalterung sind so niedrig, dass sie für die Messungen nicht geeignet sind. Je weiter die Eigenfrequenz von der zu messenden Frequenz entfernt ist, desto besser lassen sich die Signale auswerten. (Siehe auch Kapitel 
5.1). Als allgemeine Regel gilt, dass die Abtastfrequenz einer Signalmessung mindestens das 5-fache der zu messenden Frequenz betragen sollte. Dieselbe Regel sollte auch bei der Resonanzfrequenz angewandt werden. Theoretisch reicht also für eine Messung von $f=131 \mathrm{~Hz}$ eine Frequenz von $f_{\text {eigen }}=655 \mathrm{~Hz}$ aus. Die Waage mit der Steifigkeit von $10 \mathrm{~N}$ kann daher von der Messung ausgeschlossen werden. Es wird also, wie bei den Vorbeifahrtmessungen, die $20 \mathrm{~N}$-Waage verwendet. Tabelle 7.1 ist zu entnehmen, dass die Kugel mit der Karbonhalterung viel leichter ist als die jeweils gleiche Kugel mit der Aluhalterung. Daraus folgt die höhere Eigenfrequenz des Systems. Allerdings sinkt die Resonanz mit der Kugelgröße.

\subsubsection{Einfluss der Kraftwaage auf die gemessene Kraft}

Um weitere systematische Fehler der Waage zu quantifizieren, werden bei den Frequenzen $f=71 ; 110 ; 131 \mathrm{~Hz}$ die Kräfte auf die Waage einmal mit und einmal ohne angeschraubte Kugel gemessen. Eine Messung bei $f=30 \mathrm{~Hz}$ war nicht möglich, da das Signal zu gering war, um die Sinusschwingung aus dem Rauschen zu isolieren.

In Abbildung 7.7 ist die Kraftmessung bei $f=71 \mathrm{~Hz}$ für eine $R=20 \mathrm{~mm}$ Kugel im Vergleich zur Kraftmessung der Waage allein aufgetragen. Die Werte auf der linken y-Achse korrespondieren zur Messung mit der Kugel, die Werte der rechten y-Achse entsprechen der Messung ohne Kugel. Es ist deutlich zu erkennen, dass die Kraft auf die Waage ohne Kugel bereits ca. 10\% der Kraft auf die Waage mit angeschraubter Kugel entspricht.

Die bisherigen Messungen bei der Zugvorbeifahrt haben gezeigt, dass der dominante Kraftanteil $F_{A}$ sein muss. Sollte die Basset-Kraft $F_{H}$ vorhanden sein, ist ihre Amplitude höchstwahrscheinlich sehr klein, maximal im Bereich von 10\%. Wenn jedoch der systematische Fehler der Waage selbst schon bei $10 \%$ liegt kann über die Existenz einer Basset-Kraft eigentlich keine Aussage getroffen werden. Zudem existiert eine Phasenverschiebung zwischen beiden Signalen. Dies ist problematisch, da zwischen den Kräften $F_{A}, F_{D}$ und $F_{H}$ ebenfalls Phasenverschiebungen existieren und es so nicht unterscheidbar ist, ob die gemessene Phasenverschiebung in der Messtechnik oder einem weiteren Kraftanteil begründet liegt.

In Tabelle 7.2 sind die prozentualen Kraftanteile der Waage ohne Kugel im Vergleich zur Kraft mit einer $R=20 \mathrm{~mm}$-Kugel bzw. einer $R=30 \mathrm{~mm}$-Kugel eingetragen. Je kleiner die Kugel ist, desto größer ist der Einfluss der Waage selbst. Allerdings verstärkt sich bei größeren Kugeln das Problem der Resonanz (vgl. Abschnitt 7.4.1).

Die Messung der verschiedenen Kraftanteile gestaltet sich mit den immensen Einflüssen der Waage an sich als große Herausforderung, so dass zusätzlich zu den Kraftmessungen mit der Waage Kraftmessungen mithilfe von optischer Objektverfolgung, welche in Kapitel 5.2 vorgestellt wurde, durchgeführt werden. 
7. Kräfte auf Objekte in periodischen instationären Strömungen

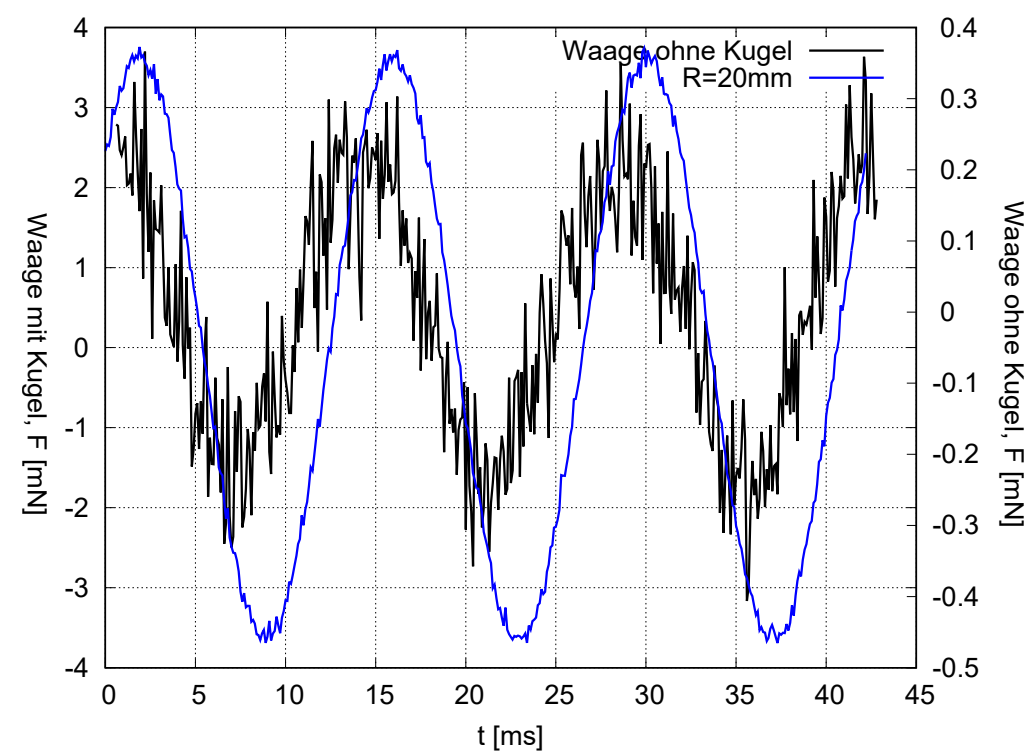

Abbildung 7.7.: Vergleich der Kraft auf die Waage mit $R=20 \mathrm{~mm}$ Kugel (blaue Kurve) und ohne angeschraubte Kugel (schwarze Kurve) bei $\mathrm{f}=71 \mathrm{~Hz}$.

\begin{tabular}{c|c|c}
$f[\mathrm{~Hz}]$ & Vgl. mit $R=20 \mathrm{~mm}[\%]$ & Vgl. mit $R=30 \mathrm{~mm}[\%]$ \\
\hline 71 & 9,78 & 3,02 \\
\hline 110 & 8,61 & 2,61 \\
\hline 131 & 7,85 & 2,44 \\
\hline
\end{tabular}

Tabelle 7.2.: Vergleich des Anteils der Kraft auf die Waage ohne Kugel im Vergleich zur jeweiligen Kugelgröße. 


\subsubsection{Durchführung der Kraftmessung}

Es werden die Frequenzen $f=30 ; 71 ; 110 ; 131 \mathrm{~Hz}$ bei den Amplituden 100; 150; 200; 250; 300; 350; $400 \mathrm{mV}$ (,peak-to-peak“) des Frequenzgenerators gemessen und die Kugeln mit den Radien $R=15 ; 20 ; 25 ; 30 \mathrm{~mm}$ verwendet. Sie werden mit der Karbonhalterung an die 20 N-Waage angeschraubt. Der Verstärker, an dem die Lautsprecherbox angeschlossen ist, befindet sich auf maximaler Einstellung. Die Geschwindigkeit der Membran wird mithilfe des Laser-Doppler-Vibrometers gemessen und mit dem Faktor aus Tabelle C.1 für die jeweilige Frequenz multipliziert, um die Geschwindigkeit auf Höhe der Kugel zu erhalten, welche sich bei $h=22,5 \mathrm{~cm}$ oberhalb des tiefsten Punktes der Lautsprechermembran befindet.

\subsubsection{Auswertung und Bestimmung der Kraftanteile}

Zunächst werden die gemessenen Signale gemittelt, in dem immer drei Schwingungen übereinander gelegt werden. Abbildung 7.8 zeigt beispielhaft das gemessene und gemittelte Geschwindigkeits- und Kraftsignal bei $f=71 \mathrm{~Hz}, R=20 \mathrm{~mm}$ und einer Amplitude von $200 \mathrm{mV}$. Das Geschwindigkeitssignal wurde bereits mit dem Normierfaktor aus Tabelle C.1 korrigiert. Bereits auf diesem Bild ist zu erkennen, dass die Kraft und die Geschwindigkeit um $90^{\circ}$ phasenverschoben sind, die Kraft und die Beschleunigung also wieder in Phase sind. Das bedeutet, dass der Hauptanteil der Kraft der zusätzlicheMasse-Kraft entspricht, da diese direkt proportional zur Beschleunigung ist.

Zunächst werden aus den gemessenen Signalen mithilfe von Gnuplot ihre jeweiligen Amplituden und Phasen für die weitere Berechnung bestimmt:

$$
\begin{aligned}
v(t) & =A_{0} \omega_{1} \sin \left(\omega_{1} t+\varphi_{1}\right), \\
\Rightarrow a(t) & =A_{0} \omega_{1}^{2} \cos \left(\omega_{1} t+\varphi_{1}\right), \\
F_{\text {Waage }}(t) & =\hat{F} \cos \left(\omega_{1} t+\varphi_{2}\right) .
\end{aligned}
$$

Hierbei handelt es sich bei $\omega_{1}$ um die Anregungsfrequenz.

Es wird angenommen, dass sich $F_{\text {mess }}(t)$ aus den einzelnen Kraftanteilen $F_{D}, F_{A}$ und $F_{H}$ zusammen setzt (siehe Gleichung 7.10):

$$
F_{\text {Waage }}(t)=F_{D}(t)+F_{A}(t)+F_{H}(t) .
$$

Für die Auswertung der gemessenen Kraftsignale und ihre Unterteilung in die einzelnen Anteile wird die Auswertemethode von Odar und Hamilton verwendet [11].

Bestimmung von $C_{D}$ : Zunächst wird die Stokes'sche Reibungskraft (engl: drag force) $F_{D}$ bestimmt.

$$
F_{D}(t)=\frac{1}{2} C_{D} \pi R^{2} A_{0}^{2} \omega_{1}^{2}\left|\sin \left(\omega_{1} t+\varphi_{1}\right)\right| \sin \left(\omega_{1} t+\varphi_{1}\right)
$$




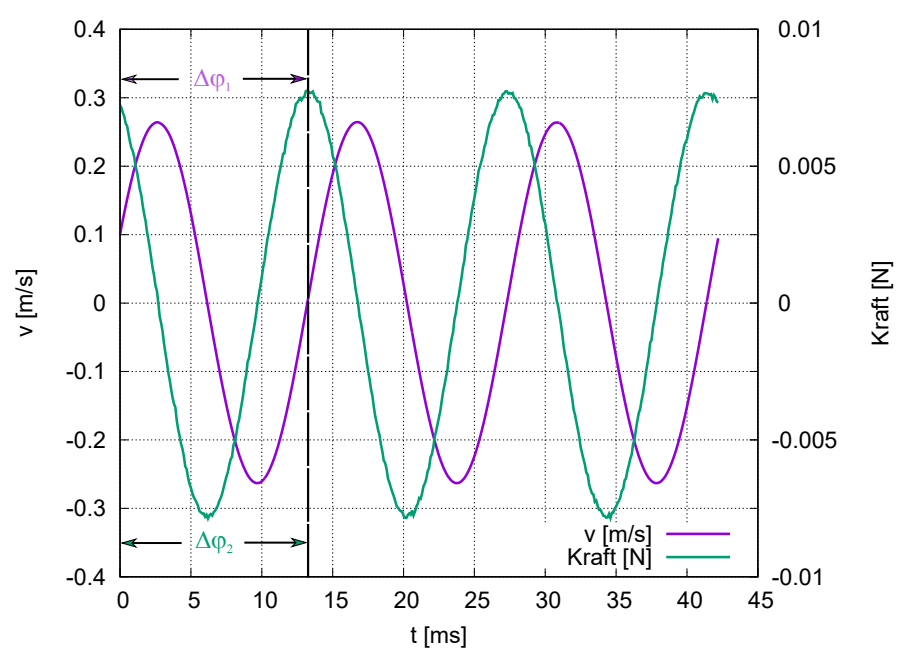

Abbildung 7.8.: Gemessene Geschwindigkeit (bereits normiert, violette Kurve) und gemessene Kraft (grüne Kurve) bei $f=71 \mathrm{~Hz}$ und der $R=20 \mathrm{~mm}$ Kugel.

Für den Widerstandskoeffizienten $C_{D}$ (dieser wird auch oft als „ $C_{W}$-Wert" bezeichnet), wird die Schiller-Naumann-Korrelation für eine Kugel verwendet [49]:

$$
C_{D}=\frac{24}{R e} \cdot\left(1+0,15 R e^{0,687}\right),
$$

mit $R e=\rho v 2 R / \mu$.

$F_{D}$ ist hiermit bestimmt.

Bestimmung von $C_{A}^{*}$ : Für die Bestimmung der zusätzliche-Masse-Kraft (engl: added mass force) wird die Stelle gesucht, an der $F_{H}=0$ ist. Da $F_{D}$ bereits bekannt ist, kann $F_{A}$ aus $F_{\text {Waage }}(t)$ und $F_{D}(t)$ an dieser Stelle bestimmt werden.

Die Bassetkraft ist

$$
F_{H}(t)=C_{H} R^{2}(\pi \mu \rho)^{\frac{1}{2}} A_{0} \omega^{3 / 2}\left(\sin \left(\omega_{1} t+\varphi_{1}\right)+\cos \left(\omega_{1} t+\varphi_{1}\right)\right) .
$$

$F_{H}=0$, wenn gilt:

$$
\sin \left(\omega_{1} t+\varphi_{1}\right)=-\cos \left(\omega_{1} t+\varphi_{1}\right) .
$$

Gleichung 7.29 ist erfüllt für

$$
\omega_{1} \cdot t_{\mathrm{mess}}=\left(\frac{3}{4} \pi-\varphi_{1}\right)
$$

Die zusätzliche-Masse-Kraft ist:

$$
\begin{aligned}
F_{A}(t) & =C_{A}^{*}\left(\pi R^{3}\right) \rho A_{0} \omega_{1}^{2} \cos \left(\omega_{1} t+\varphi_{1}\right) \\
\Rightarrow F_{A}(t) & =C_{A}^{*}\left(\pi R^{3}\right) \rho \cdot a(t) .
\end{aligned}
$$


An der Stelle $t_{\text {mess }}$ ergibt sich dann $C_{A}^{*}$ wie folgt:

$$
C_{A}^{*}=\frac{F_{\text {Waage }}\left(t_{\text {mess })}-F_{D}\left(t_{\text {mess }}\right)\right.}{\left(\pi R^{3}\right) \rho \cdot a\left(t_{\text {mess }}\right)} .
$$

Odar und Hamilton nehmen an, dass $C_{A}$ über die gesamte Schwingung konstant ist, so dass es für jedes $t$ gilt. Somit ist $F_{A}(t)$ hiermit ebenfalls bestimmt.

Bestimmung von $\boldsymbol{C}_{\boldsymbol{H}}$ : Schließlich muss nur noch die Basset-Kraft $F_{H}(t)$ bestimmt werden. Es wird angenommen, dass die restliche Kraft komplett der Basset-Kraft entspricht, also:

$$
F_{\text {Rest }}(t)=F_{\text {Waage }}(t)-F_{D}(t)-F_{A}(t) .
$$

Es sei

$$
\begin{aligned}
F_{H}(t) & =C_{H} R^{2}(\pi \mu \rho)^{\frac{1}{2}} A_{0} \omega_{1}^{3 / 2}\left(\sin \left(\omega_{1} t+\varphi_{1}\right)+\cos \left(\omega_{1} t+\varphi_{1}\right)\right), \\
\Rightarrow F_{H}(t) & =C_{H} \cdot \tilde{F}_{H}(t) .
\end{aligned}
$$

also ist

$$
C_{H}=\frac{F_{\text {Rest }}(t)}{\tilde{F}_{H}(t)} .
$$

Hiermit sind alle drei Kraftanteile bestimmt, so dass die theoretische Kraft der vibrierenden Kugel in einer gleichmäßigen Strömung lautet:

$$
F_{\text {theo }}(t)=F_{D}(t)+F_{A}(t)+F_{H}(t),
$$

mit den zuvor bestimmten Koeffizienten $C_{D}, C_{A}$ und $C_{H}$.

\subsubsection{Ergebnisse}

In Abbildung 7.9 sind die ausgewerteten Kraftanteile für eine Messung ( $f=110 \mathrm{~Hz}$, $R=20 \mathrm{~mm}$ bei einer Amplitude von $200 \mathrm{mV}$ ) aufgetragen. In Anhang $\mathrm{C}$ befinden sich die ausgewerteten Kurven für die anderen Messungen bei $200 \mathrm{mV}$. Die Ergebnisse der restlichen Messungen befinden sich in Tabelle C.2. $F_{D}$ ist so klein, dass es zu vernachlässigen ist. Auch $F_{H}$ beträgt immer maximal $10 \%$ von $F_{A}$. Das bedeutet, dass bei allen gemessenen Konfigurationen die zusätzliche-Masse-Kraft dominiert und damit den Haupteinfluss auf die Kräfte auf die Kugel in instationären Strömungen hat. Hierbei ist allerdings zu beachten, dass in Abschnitt 7.4.1 und 7.4.2 ein großer Einfluss der Kraftwaage selbst ermittelt wurde. Es ist also nicht zu unterscheiden, ob $F_{H}$ wirklich existiert, oder ob die 10\% Abweichung der Messtechnik geschuldet sind. 


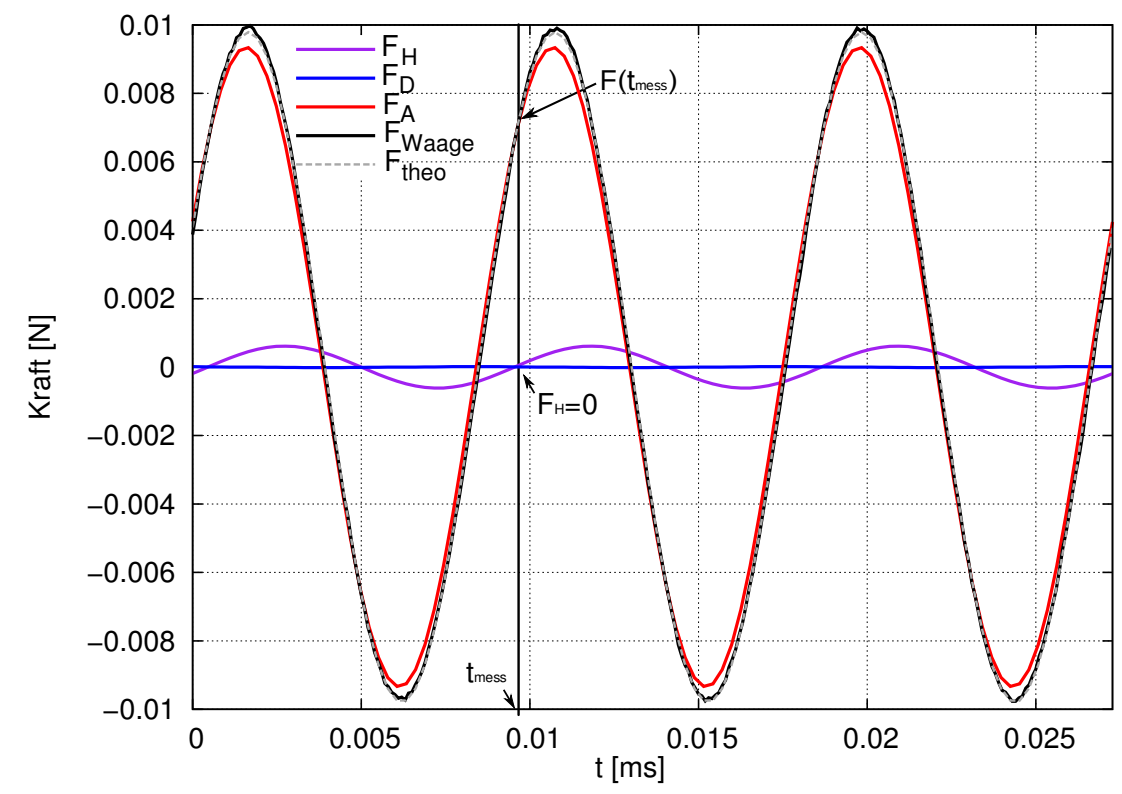

Abbildung 7.9.: Kraftanteile im Vergleich mit der gemessenen Kraft für $f=110 \mathrm{~Hz}$, $R=20 \mathrm{~mm}$ und einer Amplitude von $200 \mathrm{mV}$. Die Position $t_{\text {mess }}$ zur Bestimmung von $C_{A}^{*}$ ist ebenfalls eingezeichnet.

Untersuchung von $C_{\boldsymbol{A}}^{*}$ Odar und Hamilton untersuchten die Abhängigkeit von $C_{A}$ von der Reynolds- und von der Beschleunigungszahl [11]. Sie stellten fest, dass $C_{A}$ unabhängig von $R e$ ist. Für die Abhängigkeit von $C_{A}$ von der Beschleunigungszahl ermittelten sie folgenden empirischen Zusammenhang [50]:

$$
C_{A}=1,05-\frac{0,55}{8,33 A c^{2}+1} .
$$

In Abbildung 7.10(a) ist das ermittelte $C_{A}^{*}$ aus den Lautsprechermessungen mit der Kraftwaage über die Reynoldszahl aufgetragen. Innerhalb einer Frequenz ist $C_{A}^{*}$ unabhängig von der Reynoldszahl. Allerdings steigt $C_{A}^{*}$ mit steigender Frequenz ebenfalls an. Daraus lässt sich schließen, dass die Resonanz der Waage hier bereits die Messung beeinflusst. In Abbildung 7.10(b) ist $C_{A}^{*}$ über der Beschleunigungszahl $A_{0} / 2 R$ aufgetragen. Ein Zusammenhang nach Gleichung 7.39 lässt sich hier nicht erkennen. Offensichtlich sind alle Frequenzen größer als $f=30 \mathrm{~Hz}$ für die Auswertung unbrauchbar.

Werden nur die Messungen bei $f=30 \mathrm{~Hz}$ berücksichtigt, lässt sich eine Funktion der Form wie in Gleichung 7.39 an die Werte anpassen. Diese lautet wie folgt:

$$
C_{A(\exp , \text { Waage })}^{*}=(1,99 \pm 0,02)-\frac{(0,16 \pm 0,02)}{(873 \pm 716) A c^{2}+1} .
$$

Für die Anpassung der experimentellen Werte für $C_{A}$ von Odar und Hamilton muss die einfallende Welle hinzugerechnet werden, also $C_{A}^{*}=4 / 3 \cdot C_{A, \mathrm{OH}}+4 / 3$, so dass sich 


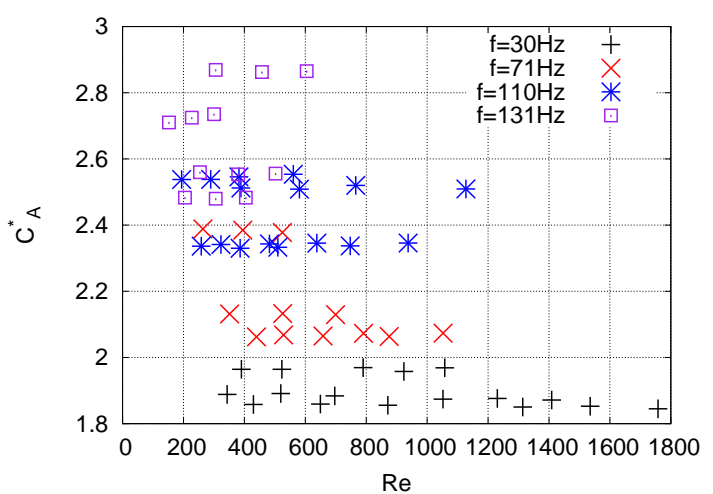

(a) $C_{A}^{*}$ aufgetragen über die Reynoldszahl

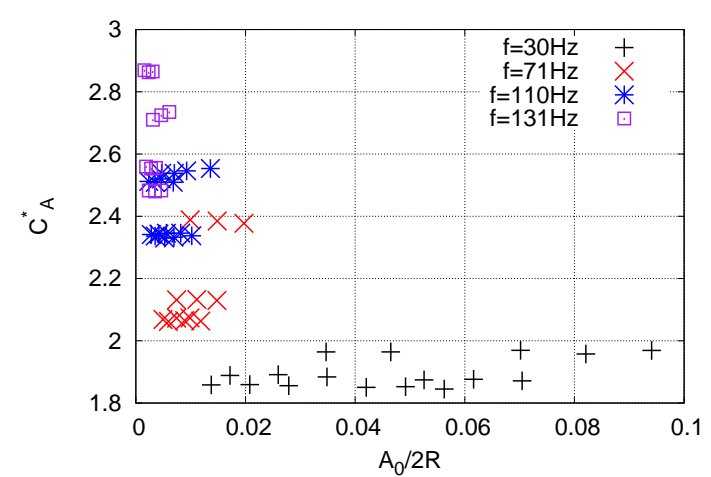

(b) $C_{A}^{*}$ aufgetragen über die Beschleunigungszahl

Abbildung 7.10.: Experimentell ermittelter Wert für $C_{A}^{*}$ für verschiedene Frequenzen.

hierfür folgende Anpassungsfunktion für $C_{A}^{*}$ ergibt:

$$
C_{A(\exp , \mathrm{OH})}^{*}=(2,66 \pm 0,02)-\frac{(0,67 \pm 0,03)}{(35 \pm 6) A c^{2}+1}
$$

Die graphischen Ergebnisse befinden sich in Abbildung 7.11. Theoretisch gilt für potentialtheoretische Strömungen $\lim _{A c \rightarrow 0} C_{A}^{*}=2$. Die Waagenmessungen unterschätzen den Wert für $C_{A}^{*}$ im kleinen $A c$-Bereich um ca. 10\%. Da der systematische Fehler der Waage selbst bereits bei $10 \%$ liegt, kann der Fehler ursächlich darin begründet liegen. Daraus ergibt sich aus der Interpolation für große $A c$ bereits ein Fehler von über 30\%. Für große Beschleunigungszahlen nähert sich $C_{A}^{*}$ einem Grenzwert an. Durch die großen Einflüsse der Waage sind diese Ergebnisse allerdings nur bedingt aussagekräftig, sodass die Kraftmessungen mithilfe von optischer Objektverfolgung als alternative Messmethode wiederholt werden.

Des Weiteren ist hinzuzufügen, dass Odar und Hamilton im kleinen Ac Bereich gar keine Messungen vorgenommen haben. Ihre Beschleunigungszahlen liegen zwischen 0,4 und 1,2. In dieser Messung ist $0<A c<0,1$, also in einem Bereich, wo nach Odar und Hamilton die Basset-Kraft $F_{H}$ durchaus relevant werden könnte. Die empirische Formel der Form von Gleichung 7.39 lässt sich jedoch dennoch anwenden, was bedeutet, dass diese auch im großen Re-Bereich gültig ist.

Die Ergebnisse zeigen, dass auch in diesem Bereich die Kraft nach wie vor von $F_{A}$ dominiert wird. Da sich $C_{A}^{*}$ schnell an einen Grenzwert annähert, genügt es daher für größere Beschleunigungs- und Reynoldszahlen, die Abhängigkeit von $C_{A}^{*}$ von $A c$ zu vernachlässigen und direkt den Grenzwert zu verwenden. Das würde zum Beispiel die instationäre Vorbeifahrt sowohl im Modell, als auch im Großmaßstab betreffen, wo die Beschleunigungszahlen entsprechend groß sind. 
7. Kräfte auf Objekte in periodischen instationären Strömungen

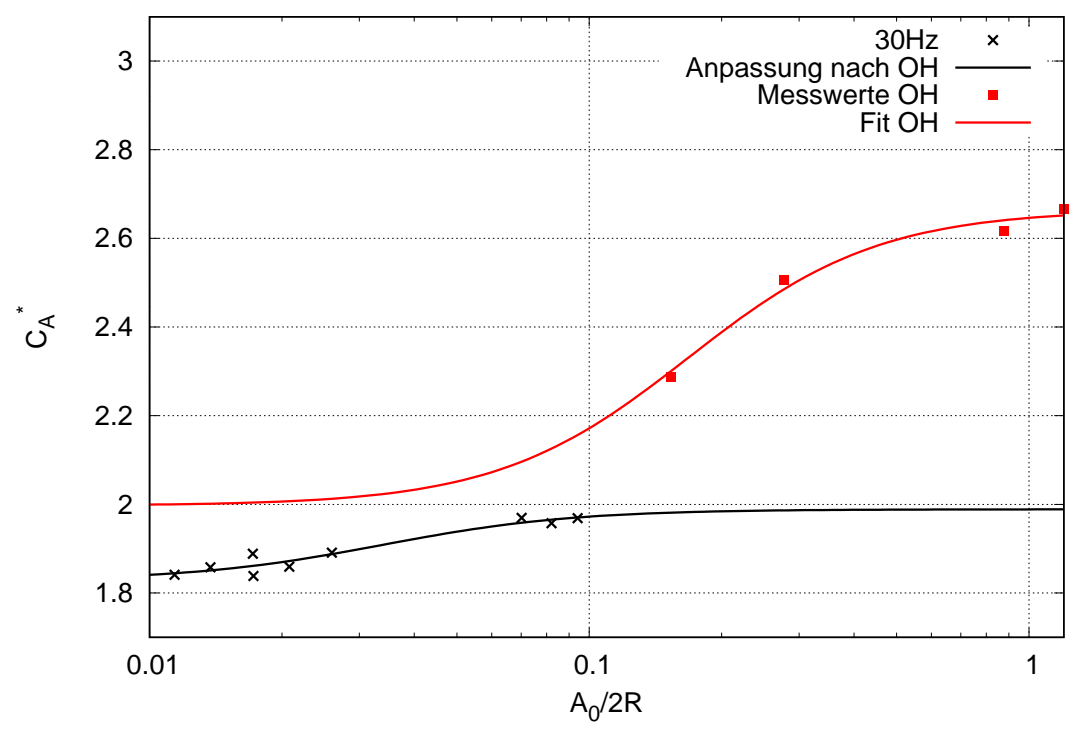

Abbildung 7.11.: Vergleich der Anpassungsfunktion nach Odar und Hamilton (OH) mit den experimentellen Werten für $C_{A}^{*}$ und den experimentellen Werten von Odar und Hamilton.

Unter Vernachlässigung von $F_{D}$ und $F_{H}$ gilt also für die Kraft auf Kugeln in instationären Strömungen folgende Gleichung:

$$
F=C_{A}^{*} \pi R^{3} \rho \frac{\partial u}{\partial t}
$$

mit $C_{A}^{*}=2$ für potentialtheoretische Strömungen und $\lim _{A c \rightarrow \infty} C_{A}^{*}=2,66$ nach Gleichung 7.41 .

Untersuchung von $\boldsymbol{C}_{\boldsymbol{H}}$ Da die Werte für $F_{H}$ kleiner sind als $10 \%$ (vgl. Tabelle C.2), der Fehler der Waage aber größer als $10 \%$ ist, kann über die Quantität von $C_{H}$ keine Aussage getroffen werden. $C_{H}$ wurde dennoch nach der Methode in Abschnitt 7.4.4 ermittelt.

In Abbildung 7.12 ist der experimentell bestimmte Wert mit der Kraftwaage für $C_{H}$ über die Reynoldszahl (Abb. 7.12(a)) und die Beschleunigungszahl (Abb. 7.12(b)) aufgetragen. Auch hier ist wieder die Abhängigkeit von der Anregungsfrequenz zu beobachten, welche in den Resonanzen der Kraftwaage an sich begründet liegt. Unter Vernachlässigung dieser Tatsache scheint auch $C_{H}$ unabhängig von der Reynoldszahl zu sein. Aufgrund der Tatsache, dass $F_{H}$ im Bereich des Fehlers der Kraftwaage liegt, kann keine quantitative Aussage getroffen werden. In Abbildung 7.12(b) ist eine große Streuung der Werte zu erkennen. Qualitativ wird $C_{H}$ kleiner für steigende $A_{0} / 2 R$.

Nach der Stokes'schen Reibungskraft muss gelten: $\lim _{A c \rightarrow 0} C_{H}=6$. Offensichtlich ist die Kraftwaage nicht geeignet, um die Basset-Kraft zu messen. Allerdings wird die Kraft auf die Kugel von der zusätzliche-Masse-Kraft $F_{A}$ dominiert, so dass die Basset-Kraft in 


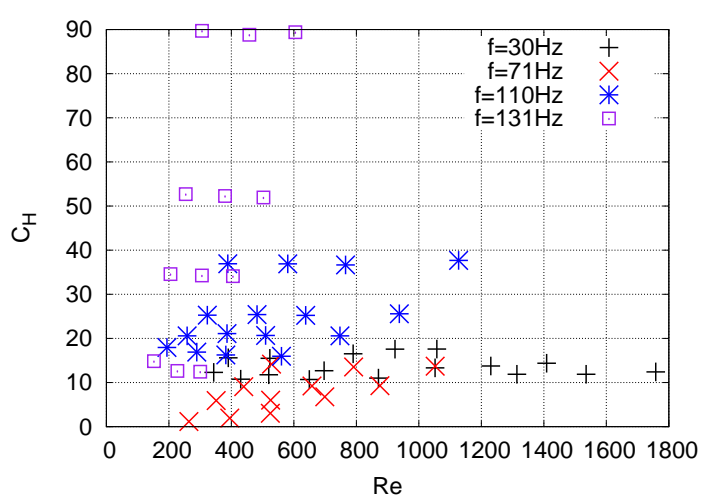

(a) $C_{H}$ aufgetragen über die Reynoldszahl

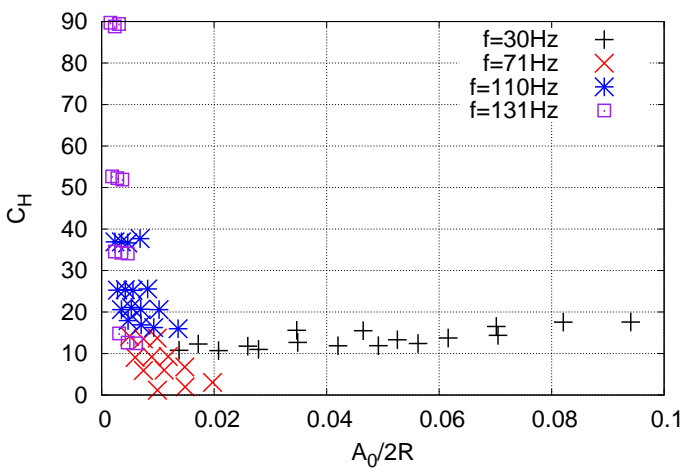

(b) $C_{H}$ aufgetragen über die Beschleunigungszahl

Abbildung 7.12.: Experimentell ermittelter Wert für $C_{H}$ für verschiedene Frequenzen.

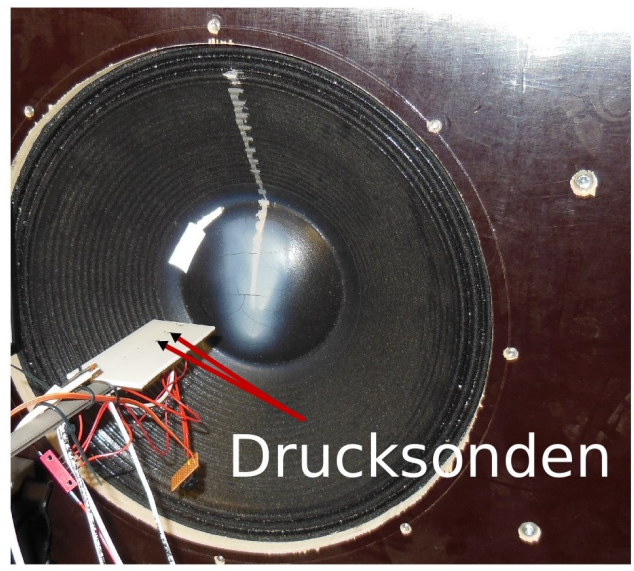

Abbildung 7.13.: Versuchsaufbau für die Messung des Druckgradienten mithife von zwei Drucksensoren im Abstand von $2 \mathrm{~cm}$.

dem gemessenen Reynoldszahlenbereich zu vernachlässigen ist. In Tabelle C.2 sind die Verhältnisse $F_{H} / F_{A}$ für alle Messungen mit der Kraftwaage eingetragen. Das Verhältnis ist nur $>10 \%$, wenn entweder eine kleine Kugel verwendet wurde, also der Einfluss der Kraftwaage selbst groß wird (vgl. Abschnitt 7.4.2), oder bei hohen Frequenzen und einer großen Kugel. In diesem Fall spielt die Eigenfrequenz des Masse-Feder-Systems bestehend aus Waage, Halterung und Kugel eine große Rolle (Vgl. Abschnitt 7.4.1). Da die Basset-Kraft einen so geringen Anteil an der Gesamtkraft hat, kann sie daher vernachlässigt werden. 
7. Kräfte auf Objekte in periodischen instationären Strömungen

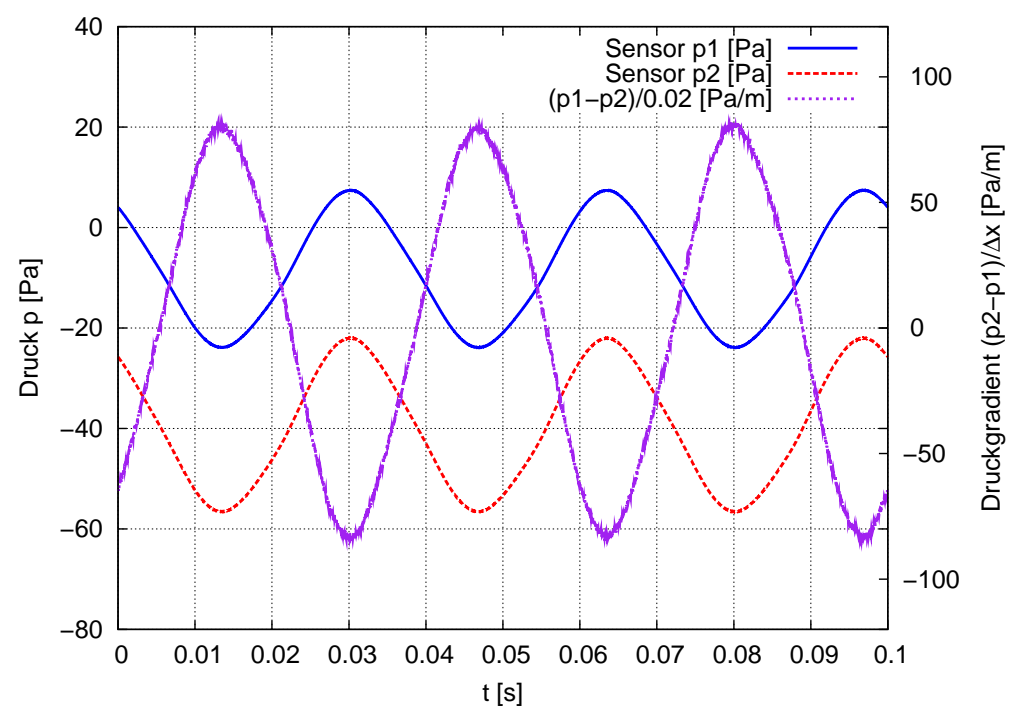

Abbildung 7.14.: Gemessene Drucksignale und daraus berechneter Druckgradient bei $f=30 \mathrm{~Hz}$ und einer Amplitude von $500 \mathrm{mV}$.

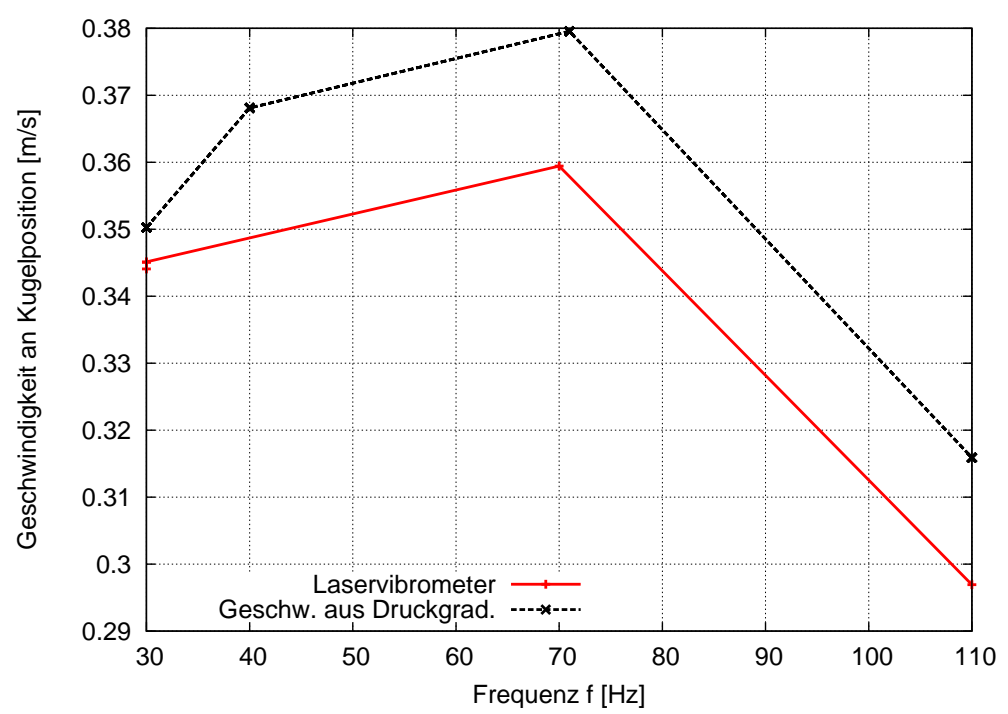

Abbildung 7.15.: Vergleich der Frequenz-Geschwindigkeits-Spektren der Drucksensoren und des Laservibrometers bei einer Amplitude von $500 \mathrm{mV}$ an der Kugelposition. 


\subsection{Messung des Druckgradienten an der Kugelposition}

Für die Kraftmessung mithilfe von optischer Objektverfolgung wird der Druckgradient und damit indirekt die Geschwindigkeit mithilfe von Drucksensoren der Firma Endevco [51] gemessen. Nach der Euler-Gleichung gilt für den Druckgradienten:

$$
\nabla p=\rho \frac{\partial u}{\partial t}
$$

Da die Anregung sinusförmig ist, lässt sich zwischen Druckgradient, Geschwindigkeit und Beschleunigung umrechnen:

$$
\begin{aligned}
u(t) & =A_{0} \cdot \sin (\omega t) \\
a(t) & =\frac{\partial u}{\partial t}=A_{0} \cdot \omega \cos (\omega t) \\
\nabla p=\hat{p} \cos (\omega t) & =\rho \frac{\partial u}{\partial t}=\rho \cdot A_{0} \cdot \omega \cos (\omega t)
\end{aligned}
$$

Der Vorteil der direkten Messung des Druckgradienten liegt darin begründet, dass dieser direkt an der gewünschten Stelle gemessen werden kann. Eine Fotografie des Versuchsaufbaus befindet sich in Abbildung 7.13. In einer Halterung sind zwei Endevco Drucksensoren im Abstand von $\Delta x=2 \mathrm{~cm}$ angebracht. Die Halterung wird so vor dem Lautsprecher positioniert, dass sich der erste Drucksensor genau auf der Position des Kugelmittelpunktes bei der späteren Kraftmessung befindet, also bei $h=22,5 \mathrm{~cm}$, gemessen vom tiefsten Punkt der Lautsprechermembran. Der Druckgradient in x-Richtung, also vom Lautsprecher weg, ergibt sich durch die Differenz der Signale der beiden Drucksonden geteilt durch ihren Abstand voneinander. Allerdings können die Kraft und der Druckgradient bei dieser Methode nicht gleichzeitig gemessen werden.

Abbildung 7.15 zeigt das Frequenz-Geschwindigkeits-Spektrum bei einer Amplitude von $500 \mathrm{mV}$ (,peak-to-peak“) an der Kugelposition des Laservibrometers (bereits auf die Höhe korrigiert) und der Druckgradientenmessung. Die Geschwindigkeit mittels Druckgradientenmessung ist im Schnitt 5\% höher als die gemessene Geschwindigkeit des Laservibrometers. Diese Abweichung liegt noch innerhalb der Fehlertoleranz, welcher bei der Waage je nach verwendeter Kugel, mehr als $10 \%$ beträgt.

\subsection{Messung der Kraft auf eine Kugel mithilfe von optischer Objektverfolgung}

\subsubsection{Aufbau und Durchführung}

Da die Eigenfrequenzen der Waage offensichtlich eine zu große Rolle spielen, wird die alternative Kraftmessmethode mithilfe der optischen Kraftmesstechnik (vgl. Kap. 5.2) verwendet. Eine Fotografie des Versuchsaufbaus befindet sich in Abbildung 7.16. Es wird die Phantom v1210 Hochgeschwindigkeitskamera mit einem UV NIKKOR 105 mm 1:4,5 


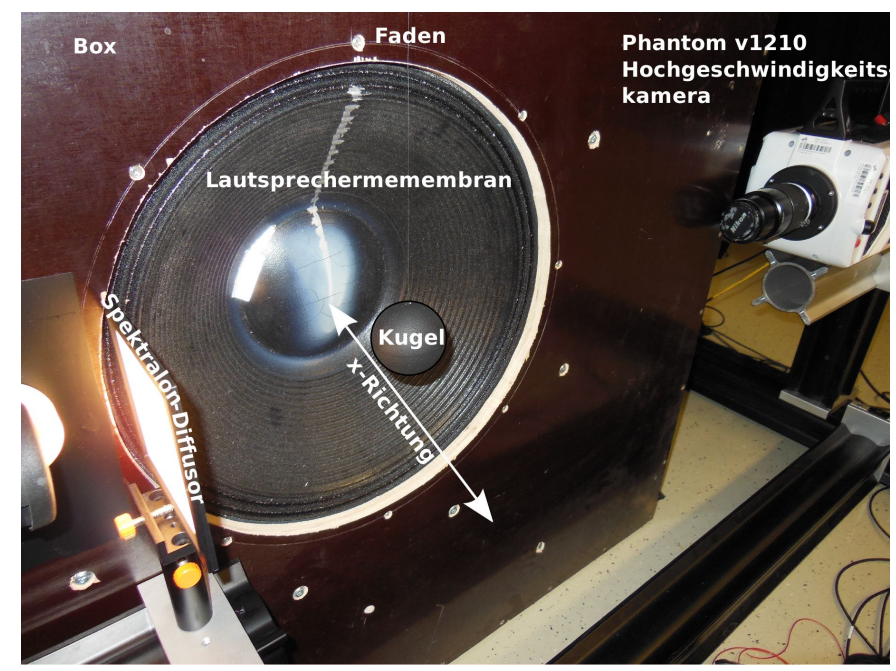

Abbildung 7.16.: Versuchsaufbau für die Kraftmessung im Lautsprecherfeld mithilfe von optischer Objektverfolgung.

C-Mount Objektiv verwendet. Die Kugel, deren Bewegung mit der Kamera verfolgt werden soll, ist an einem dünnen Faden befestigt. Dieser wird so oberhalb des Lautsprechers befestigt, dass sich der Kugelmittelpunkt genau wie bei den Messungen mit der Kraftwaage bei einem Abstand von $h=22,5 \mathrm{~cm}$ vom tiefsten Punkt der Lautsprechermembran befindet. Die verwendete Diffusorplatte der Firma Laser2000 hat die Maße 133x133 mm² und ist 0,18 mm dick. Bei dem Material handelt es sich um „Spektralon“. Es hat viele Lufteinschlüsse, daher verhält es sich bezüglich der Lichtstreuung nicht wie ein gewöhnlicher Festkörper. Spektralon hat aufgrund der geringen Dicke ein hohes Transmissionsvermögen und zeigt bei Durchlicht keinerlei Strukturen, im Gegensatz zu Papier. Die Messungen werden bei den Anregungsfrequenzen $f=30 ; 40 ; 71 ; 110 ; 131 ; 200 \mathrm{~Hz}$, den Amplituden des Frequenzgenerators 200;500;1000 mV und mit den Kugeln der Radien $R=15 ; 20 ; 25 ; 30 \mathrm{~mm}$ durchgeführt. Die Aufnahmefrequenz der Kamera ist $f_{\text {Aufn }}=1 \mathrm{kHz}$ bei den Frequenzen $f=30 ; 40 ; 71 \mathrm{~Hz}$ und $f_{\text {Aufn }}=5 \mathrm{kHz}$ bei den Frequenzen $f=110 ; 131 ; 200 \mathrm{~Hz}$. Die Kugeln werden mit schwarzer Acrylfarbe versehen, damit ein hoher Kontrast vor der Diffusorplatte gewährleistet ist und die Kantendetektion gut funktioniert.

\subsubsection{Auswertung}

Die aufgenommenen Bilder werden auf die selbe Art und Weise ausgewertet, wie in Abschnitt 6.4.2 beschrieben, so dass man am Ende eine ASCII-Datei mit der Position des Kugelmittelpunktes für jeden Zeitschritt, sowie den Radius der Kugel in Pixeln erhält. Bei diesen Messungen wird nur die Bewegung in x-Richtung, also vertikal zur Lautsprechermembran betrachtet.

In Abbildung 7.17 ist exemplarisch für eine Messung bei $f=30 \mathrm{~Hz}$ und einer Amplitude 


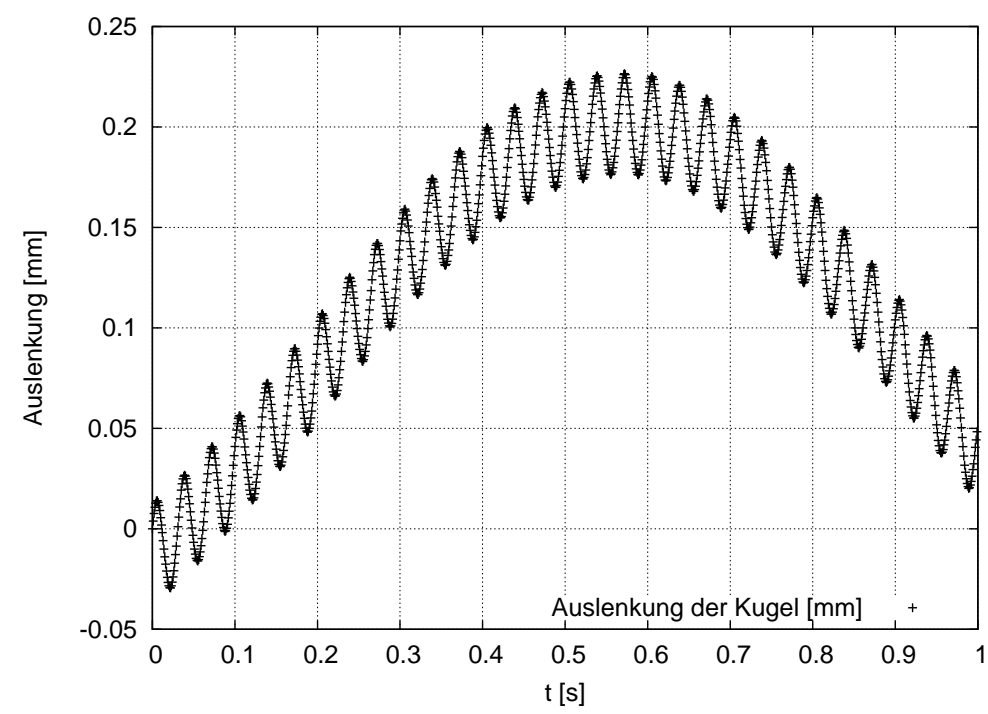

Abbildung 7.17.: Verfolgte Bewegung der $R=15 \mathrm{~mm}$-Kugel bei $f=30 \mathrm{~Hz}$ und einer Amplitude von $1000 \mathrm{mV}$.

\begin{tabular}{c|c} 
Kugelradius $[\mathrm{mm}]$ & Masse $[\mathrm{g}]$ \\
\hline 15 & $0,45 \pm 0,01$ \\
\hline 20 & $0,80 \pm 0,01$ \\
\hline 25 & $1,50 \pm 0,01$ \\
\hline 30 & $2,55 \pm 0,01$ \\
\hline
\end{tabular}

Tabelle 7.3.: Massen der verwendeten Kugeln. 
von1000 mV für die $R=15 \mathrm{~mm}$-Kugel die Auslenkung der Kugel über die Zeit aufgetragen. Die kleinen Schwingungen entsprechen der jeweiligen Anregungsfrequenz, die große Oberschwingung wird durch die Pendelbewegung der Kugel am Faden verursacht. Da diese aber im Bereich von $\approx 1 \mathrm{~Hz}$ liegt, beeinflusst sie die Messung nicht. Dennoch wird sie bei der Auswertung berücksichtigt.

Die Bewegungsgleichung der Kugel lautet daher wie folgt:

$$
x_{K}(t)=A_{1} \cos \left(\omega_{1} t+\varphi_{1}\right)+A_{2} \cos \left(\omega_{2} t+\varphi_{2}\right) .
$$

Bei den Variablen mit dem Index 1 handelt es sich um die Schwingung, welche durch die Anregungsfrequenz ausgelöst wird. Die Variablen mit dem Index 2 sind der Pendelschwingung zugeordnet. Daraus ergeben sich dann durch Ableitung die Geschwindigkeit und die Beschleunigung der Kugel:

$$
\begin{aligned}
& u_{K}(t)=-A_{1} \omega_{1} \sin \left(\omega_{1} t+\varphi_{1}\right)-A_{2} \omega_{2} \sin \left(\omega_{2} t+\varphi_{2}\right), \\
& a_{K}(t)=-A_{1} \omega_{1}^{2} \cos \left(\omega_{1} t+\varphi_{1}\right)-A_{2} \omega_{2}^{2} \cos \left(\omega_{2} t+\varphi_{2}\right) .
\end{aligned}
$$

Die Kraft auf die Kugel berechnet sich aus der Kugelmasse multipliziert mit der Beschleunigung. Die Kugelmassen für die verschiedenen Bälle sind in Tabelle 7.3 eingetragen.

$$
F_{K}(t)=m_{K} \cdot a_{K}(t) .
$$

Aus technischen Gründen war es nicht möglich, das Druck- und das Kraftsignal zeitlich zu synchronisieren, so dass es möglich wäre die Basset-Kraft $F_{H}$ aus der Phasenverschiebung zwischen Kraft und Druckgradient zu bestimmen. Da die Ergebnisse aus Abschnitt 7.4.5 gezeigt haben, dass der Anteil der Basset-Kraft kleiner als 10\% ist (vgl. Tab C.2), kann diese hier vernachlässigt werden, so dass hier lediglich die zusätzliche-Masse-Kraft $F_{A}$ mit dem Koeffizienten $C_{A}^{*}$ untersucht wird. Die Ergebnisse aus Abschnitt 7.4.5 haben eine große Abhängigkeit von der Anregungsfrequenz gezeigt, welche vermutlich in der verwendeten Kraftmesstechnik begründet liegt. $C_{A}^{*}$ wird daher mithilfe der Kraftmessung mittels optischer Objektverfolgung erneut untersucht.

Für die zusätzliche-Masse-Kraft $F_{A}$ wird folgende Funktion an die Gleichung 7.50 angepasst und somit schließlich $C_{A}^{*}$ ermittelt:

$$
F_{A}=C_{A}^{*} \cdot \pi R^{3} \rho A_{0} \cos \left(\omega_{1} t+\varphi_{3}\right),
$$

mit

$$
A_{0}=\frac{\hat{p}}{\rho \omega_{1}} .
$$

\subsubsection{Ergebnisse}

Im Anhang in Tabelle C.3 sind die Ergebnisse der Kraftmessung mithilfe von optischer Objektverfolgung eingetragen. Abbildung 7.18 zeigt exemplarisch für $f=30 \mathrm{~Hz}$ und $A m p=1000 \mathrm{mV}$ für die $R=15 \mathrm{~mm}$-Kugel die berechnete Kraft aus der gemessenen Bewegungstrajektorie der Kugel und die daraus ermittelte zusätzliche-Masse-Kraft $F_{A}$. 


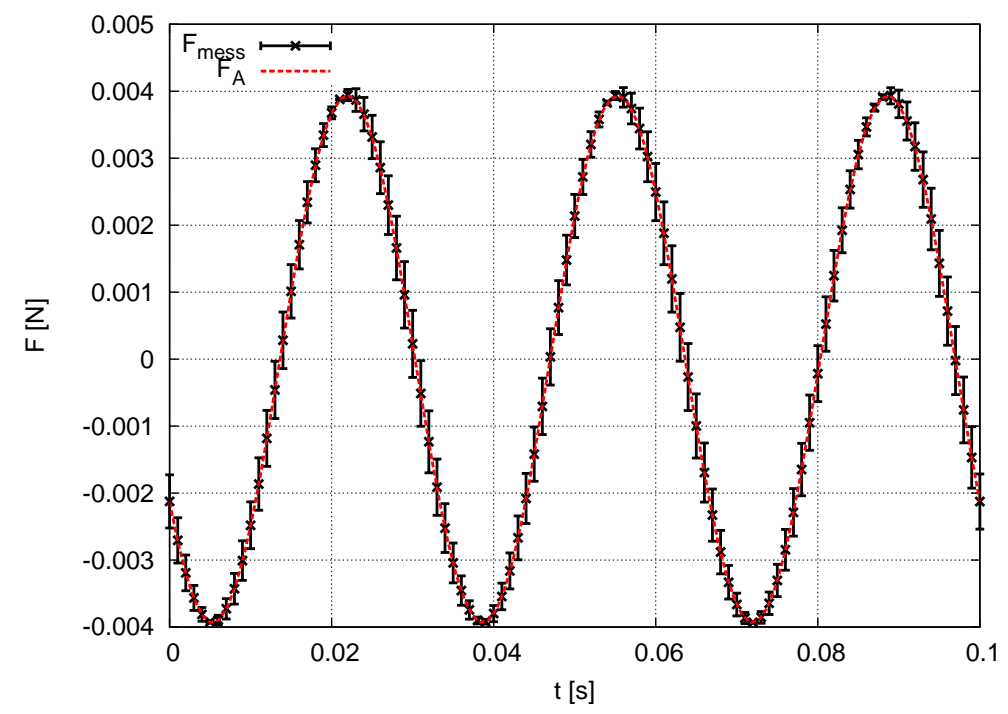

Abbildung 7.18.: Berechnete Kraft aus der gemessenen Kugeltrajektorie mittels optischer Objektverfolgung bei $f=30 \mathrm{~Hz}, A m p=1000 \mathrm{mV}, R=15 \mathrm{~mm}$.

Die Fehler für die gemessene Kraft ergeben sich wie in Abschnitt 6.4.2 mithilfe der Gauß'schen Fehlerfortpflanzung:

$$
\begin{aligned}
\sigma_{F}(i) & =\sqrt{\sigma_{m}^{2}\left(\frac{\partial F}{\partial m_{K}}\right)^{2}+\sigma_{x}^{2}(i)\left(\frac{\partial F}{\partial \Delta x(i)}\right)^{2}+\sigma_{t}^{2}\left(\frac{\partial F}{\partial \Delta t}\right)^{2}} \\
\Rightarrow \sigma_{F}(i) & =\sqrt{\sigma_{m_{K}}^{2}\left(\frac{\Delta x(i)}{\Delta t^{2}}\right)^{2}+\sigma_{x}^{2}(i)\left(\frac{m_{K}}{\Delta t^{2}}\right)^{2}+\sigma_{t}^{2}\left(-\frac{2 m_{K} \Delta x(i)}{\Delta t^{3}}\right)^{2}},
\end{aligned}
$$

mit $m_{K}$ als Kugelmasse (siehe Tabelle 7.3) und $\Delta t=1 / f_{A u f n}$. Der Fehler der Bewegung $\sigma_{x}$ wird für jeden Film erneut berechnet und ergibt sich aus dem mithilfe des Algorithmus bestimmten Kugelradius in Pixeln und dessen Standardabweichung $\sigma_{R}$.

Abbildung 7.19 zeigt die Anpassung der Werte für $C_{A}^{*}$ nach der empirischen Funktionsgleichung von Odar und Hamilton. Es ergibt sich folgende Funktion für die Ergebnisse aus der optischen Objektverfolgung:

$$
C_{A(\exp , \text { optisch })}^{*}=(2,34 \pm 0,03)-\frac{(0,42 \pm 0,04)}{(636 \pm 293) A c^{2}+1} .
$$

Die Ergebnisse der Messungen mit der Kraftwaage aus Abschnitt 7.4.5, sowie die empirische Funktion nach den Messwerten von Odar und Hamilton sind ebenfalls mit eingezeichnet.

Aus den Kraftmessungen mittels optischer Objektverfolgung werden höhere Werte für $C_{A}^{*}$ ermittelt, als aus den Waagenmessungen. Da die empirische Funktion von Odar und Hamilton ebenfalls höhere Werte vorhersagt, und $C_{A}^{*}=2$ sein muss für kleine $A c$, wird 


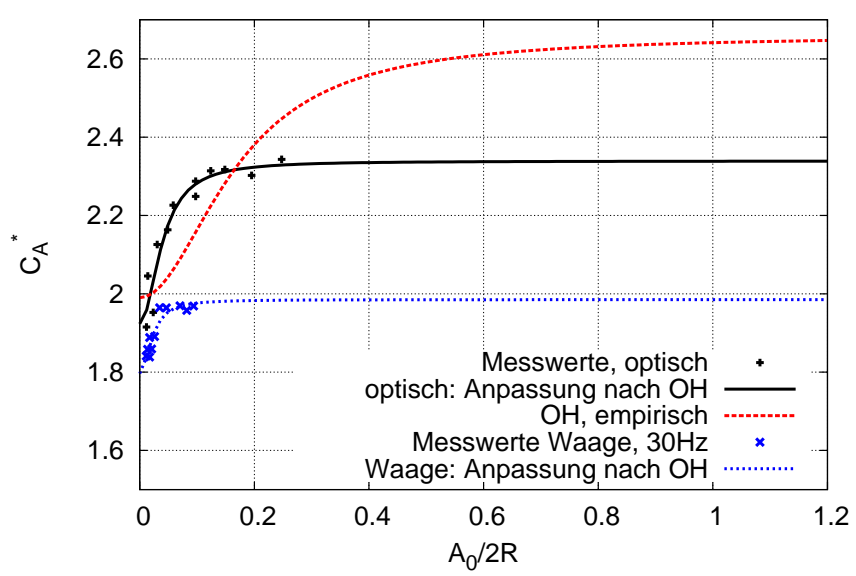

Abbildung 7.19.: Vergleich der Messwerte und angepassten Funktionsgleichungen für $C_{A}^{*}$ in Abhängigkeit von $A c$, ermittelt mit der Kraftwaage sowie der optischen Objektverfolgung mit der empirischen Funktion von Odar und Hamilton $(\mathrm{OH})$.

deutlich, dass die Messungen mit der Kraftwaage ein viel zu niedriges $C_{A}^{*}$ ermitteln.

Aus den optischen Messungen ergibt sich $\lim _{A c \rightarrow \infty} C_{A}^{*}=(2,34 \pm 0,03)$. Der Verlauf der Kurve von Odar und Hamilton passt im kleinen $A c$-Bereich weder zu den Waagenmessungen noch zu den optischen Messungen, allerdings haben sie genau in diesem Bereich auch keine Messwerte. Da der Verlauf der Kurve sowohl für die Waagenmessungen als auch für die optischen Messungen qualitativ gleich verläuft, ist davon auszugehen, dass Odar und Hamilton diesen Bereich mit ihrer Funktion nicht richtig einschätzen. Ihre Funktion ist lediglich eine Anpassung der gemessenen Werte, die ja nur im großen $A c$ Bereich existieren.

Da sich $C_{A}^{*}$ bereits ab einem Wert für $A c=0,2$ dem Grenzwert von $C_{A}^{*}=2,34$ annähert, kann vereinfachend für die Kraft auf eine Kugel in einem instationären Strömungsfeld die folgende Gleichung verwendet werden:

$$
F=2,34 \pi R^{3} \nabla p \text {. }
$$

Der potentialtheoretische Kraftanteil dominiert also nach wie vor die Kraft auf die Kugel, allerdings gilt in einer nicht schleichenden und nicht potentialtheoretischen Strömung nicht mehr der Wert $C_{A}^{*}=2$, sondern ein Wert, welcher von der Auslenkung der Luft $A_{0}$, sowie dem Kugelradius $R$ abhängt.

\subsection{Diskussion}

Die Messungen der Kraft auf eine Kugel im instationären Feld eines Lautsprechers haben gezeigt, dass über einen großen Reynoldszahlenbereich sowohl die Stokes'sche Reibungskraft $F_{D}$ als auch die Basset-Kraft $F_{H}$ so klein sind, dass sie zu vernachlässigen sind. 
Ablösung spielt in diesen Bereichen ebenfalls noch keine Rolle, da eine Kugel etwa 2/5 ihres Radius zurücklegen muss, bevor Ablösung beginnt [52]. Dies entspricht für eine Kugel mit einem Radius von $R=15 \mathrm{~mm}$ einer Strecke von $6 \mathrm{~mm}$. In Abbildung 7.17 ist zu erkennen, dass die Kugel noch nicht mal 1/100 dieser Strecke zurücklegt.

Die Kraft auf eine Kugel in instationären Strömungen hängt also maßgeblich von ihrer Beschleunigung ab, so dass die zusätzliche-Masse-Kraft $F_{A}$ die Kraft auf die Kugel dominiert.

Das Kraftmesselement war für die Messungen ungeeignet, da dessen Eigenfrequenzen eine zu große Rolle spielen. Die Ergebnisse zeigen eine Frequenzabhängigkeit, die bei den Messungen mittels optischer Objektverfolgung nicht mehr vorhanden sind. Umgekehrt waren Messungen bei einer niedrigen Frequenz $(f=30 \mathrm{~Hz})$ und der $R=15 \mathrm{~mm}$ Kugel sehr ungenau, da sich das Signal kaum mehr vom Rauschen unterscheiden ließ. Bei der optischen Objektverfolgung war dies kein Problem, da durch die hohe Auflösung im Bereich von unter $500 \mu \mathrm{m} / \mathrm{px}$ auch sehr kleine Bewegungen noch ausreichend gut verfolgt werden konnten.

In Kapitel 2.3 wurde hergeleitet, dass die potentialtheoretische Kraft auf eine Kugel ausgedrückt werden kann als:

$$
F=C_{A}^{*} \cdot \pi R^{3} \nabla p,
$$

mit $C_{A}^{*}=2$. Die Messungen haben gezeigt, dass dieser Wert nur für schleichende Strömungen oder für Potentialströmungen korrekt ist. Für eine größere Beschleunigungszahl $A c$, welche als das Verhältnis zwischen Auslenkung der Luft und dem Durchmesser der Kugel ausgedrückt werden kann, ergibt sich eine Abhängigkeit von Ac. Allerdings wird schnell ein Grenzwert erreicht, so dass die Kraft vereinfacht ausgedrückt werden kann als:

$$
F=2,34 \cdot \pi R^{3} \nabla p
$$

Werden diese Ergebnisse nun wieder auf das Problem der instationären Strömungen, welche durch Zugvorbeifahrten entstehen, übertragen, ergeben sich die folgenden Überlegungen: Im 1:25 Modell ergibt sich eine Geschwindigkeit neben dem Zug von $v \approx 6 \mathrm{~m} / \mathrm{s}$ (vgl. Abschnitt 4). Die Frequenz der Kraftkurve beträgt $f \approx 300 \mathrm{~Hz}$. Daraus ergibt sich für eine $R=20 \mathrm{~mm}$ Kugel mit $A_{0}=v /(2 \pi f)$ eine Beschleunigungszahl von $A_{0} / 2 R=0,06$.

Im Originalmaßstab darf laut der TSI-Norm [13] die Geschwindigkeit am Gleis von $v=15,5 \mathrm{~m} / \mathrm{s}$ nicht überschritten werden, daher wird diese Geschwindigkeit für $v$ angenommen. Die Frequenz der Kraftkurve beträgt bei einem Zug der Fahrtgeschwindigkeit $u_{\infty}=200 \mathrm{~km} / \mathrm{h}$ in etwa $f \approx 20 \mathrm{~Hz}$. Bei einer 25 -mal größeren Kugel, also $R=0,5 \mathrm{~m}$ ergibt sich für $A c=A_{0} / 2 R \approx 0,12$. Also wurden in der instationären Kraftmessung im Lautsprecherfeld sowohl die Verhältnisse für das 1:25-Modell, als auch für Züge im Originalmaßstab abgedeckt.

Für größere Kugeln wird die Beschleunigungszahl kleiner, demzufolge auch die Zahl $C_{A}^{*}$. 


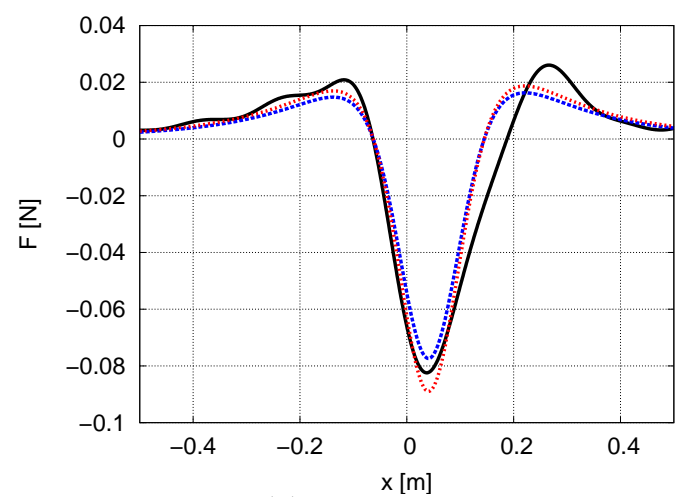

(a) x-Richtung

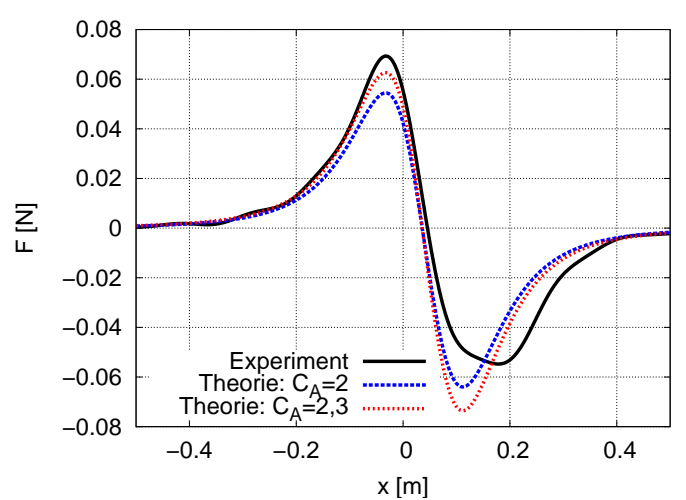

(b) y-Richtung

Abbildung 7.20.: Vergleich der gemessenen Kraft auf eine Kugel $(R=20 \mathrm{~mm})$ bei der Vorbeifahrt $\left(u_{\infty}=40 \mathrm{~m} / \mathrm{s}, y_{M}=12 \mathrm{~cm}\right)$ im Vergleich mit der potentialtheoretischen Kraftvorhersage mit $C_{A}^{*}=2$ und $C_{A}^{*}=2,3$.

Eine Verringerung von $C_{A}^{*}$ von 2,3 auf 2,1 beträgt allerdings nur gut $10 \%$. Aus sicherheitsrelevanter Sicht wäre es von daher sinnvoll den Wert bei $C_{A}^{*}=2,3$ zu belassen und die wirkende Kraft auf die Kugel mithilfe der vereinfachten Formel 7.58 zu berechnen. Selbst wenn die Kraft ca. $10 \%$ zu hoch eingeschätzt würde, ist das Vorhersagemodell für die wirkende Kraft dadurch immer noch deutlich genauer und näher an der Realität für komplexere Zuggeometrien als das bisher in der Literatur verwendete Modell der rotationssymmetrischen Halbkörper.

In Abschnitt 6 wurden die Kräfte bei der Vorbeifahrt auf Kugeln gemessen und mit der potentialtheoretischen Kraft (Gleichung 7.57) mit $C_{A}^{*}=2$ verglichen. Die theoretische Kraftamplitude unterschätzte die gemessene Kraftamplitude leicht. In Abbildung 7.20 sind nun die gemessenen Kraftkurven in Fahrtrichtung des Zuges (x-Richtung), sowie lateral dazu (y-Richtung) aufgetragen. Es handelt sich um die Messung mit der Kugel des Radius $R=20 \mathrm{~mm}$, der Fahrtgeschwindigkeit $u_{\infty}=40 \mathrm{~m} / \mathrm{s}$ bei einem Gleisabstand vom Mittelpunkt der Kugel zur Mitte vom Gleis von $y_{M}=12 \mathrm{~cm}$. Zum Vergleich sind die theoretischen Kräfte einmal mit $C_{A}^{*}=2$ und einmal mit $C_{A}^{*}=2,3$ berechnet und eingezeichnet worden. Es ist zu erkennen, dass die Vorhersagegenauigkeit der gemessenen Kraftamplitude um $50 \%$ verbessert wird, wenn für $C_{A}^{*}=2,3$ anstatt $C_{A}^{*}=2$ eingesetzt wird. 
KAPITEL 8

\section{Zusammenfassung und Ausblick}

In bisherigen Veröffentlichungen wurde gezeigt, dass unter Annahme der Reibungsfreiheit des betrachteten Fluides, die Potentialtheorie als adäquate Methode herangezogen werden kann, um wirkende Kräfte im instationären Strömungsfeld eines Zugkopfes vorherzusagen. Es wurden jedoch weder reale Zuggeometrien noch unterschiedliche Körperformen berücksichtigt. Es ist daher nicht einzugrenzen, wo die Grenzen der Potentialtheorie liegen und bei welchen Körpern weitere physikalische Effekte, wie eben beispielsweise die Reibung relevant werden. Die genaue Kenntnis der physikalischen Effekte ist notwendig, um möglichst genaue Vorhersagen bezüglich der Gefahren für Personen in der Nähe eines vorbeifahrenden Zuges treffen zu können. Des Weiteren ist es nur bedingt möglich, Messungen mit benötigter Genauigkeit im Originalmaßstab durchzuführen. Daher wäre es für die Entwicklung von neuen Zuggeometrien günstig, wenn die aerodynamisch induzierten Geschwindigkeiten und wirkende Kräfte auf Körper in Gleisnähe durch einen vorbeifahrenden Zug bereits in der Entwurfsphase in Modellexperimenten überprüft werden könnten.

In dieser Arbeit wurde ein analytisches Modell hergeleitet, welches die aerodynamischen Lasten auf einen Körper in instationärer Strömung, induziert durch die Kopfwelle eines vorbeifahrenden Zuges sowohl für Modellexperimente als auch für den Originalmaßstab vorhersagen kann.

Zur Validierung dieses analytischen Modells wurden Modellexperimente im Maßstab 1:25 an der Tunnel-Simulations-Anlage Göttingen (TSG) am Deutschen Zentrum für Luft- und Raumfahrt (DLR) durchgeführt. Es wurden drei verschiedene Zuggeometrien verwendet: ein ICE3-Modell, ein NGT-Modell, sowie ein Potentialzugmodell. Geschwindigkeitsmessungen mittels Hitzdraht-Anemometrie haben gezeigt, dass die Strömung um den Potentialzug der mit dem analytischen Modell vorhergesagten Strömung entspricht. 


\section{Zusammenfassung und Ausblick}

Die Kräfte auf Kugeln und Scheiben wurden mithilfe eines eindimensionalen piezoresistiven Kraftmesselementes gemessen. Es zeigte sich bei den Kugeln eine sehr gute Übereinstimmung mit der Potentialtheorie mit leichten Abweichungen in der Amplitude und der Phase. Die Abweichungen in der Phase konnten durch Messungen in verschiedenen Höhen der Kugel über der Schienenoberkante geklärt werden. Sie entstehen vermutlich durch von der Unterbodenströmung des Zuges erzeugten Verwirbelungen. Diese Bodeneffekte werden nicht in der Potentialtheorie berücksichtigt. Ab einer Kugelposition auf halber Zughöhe stimmen die Kräfte aus Theorie und Experiment überein.

Zur Überprüfung der Messtechnik wurden weitere, nicht-intrusive Kraftmessungen durch optische Objektverfolgung der Kugel durchgeführt. Diese zeigten eine geringere Abweichung gegenüber der Potentialtheorie bezüglich Amplitude und Phase als die Messungen mit der Kraftwaage. Die durch die optische Objektverfolgung bestimmten Kräfte sind systematisch kleiner als die Vorhersage-Werte der Potentialtheorie und die Messungen des Kraftelementes liegen systematisch über den potentialtheoretischen Werten.

Um die Abweichungen zwischen den Experimenten und der Potentialtheorie weiter zu untersuchen, wurden anschließend Kraftmessungen im instationären Feld eines Lautsprechers durchgeführt. Für die Kräfte auf Kugeln in periodisch instationären Strömungen existiert eine Erweiterung zur Potentialtheorie, bisher jedoch nur für schleichende Strömungen, also Strömungen mit kleinen Geschwindigkeiten und demzufolge kleinen Reynoldszahlen. Bei der Zugvorbeifahrt treten hingegen hunderttausendfach größere Reynoldszahlen auf.

Basset [4], Boussinesq [5] und Oseen [6] leiteten eine Gleichung ab (BBO-Gleichung), in der neben dem potentialtheoretischen Term sowohl die Stokes'sche Reibungskraft als auch die so genannte „Basset-Kraft" auftreten. Letztere berücksichtigt zusätzlich die zeitliche Änderung der Beschleunigung. Experimentelle Untersuchungen wurden bisher lediglich von Odar und Hamilton $(\mathrm{OH})$ durchgeführt [11]. Sie gewichteten die einzelnen Terme der BBO-Gleichung mit experimentell bestimmten Faktoren. Sie führten ihre Experimente jedoch wieder nur für kleine Reynoldszahlen bis $R e=62$ durch.

Die Experimente in der vorliegenden Arbeit decken einen bisher nicht gemessenen Bereich von $R e=200$ bis $R e=1800$ ab. Sie haben gezeigt, dass der potentialtheoretische Kraftanteil, die so genannte „zusätzliche-Masse-Kraft" die Kraft auf die Kugel in allen gemessenen Fällen dominiert. Die erhaltenen Ergebnisse lassen sich auf die Zugvorbeifahrt sowohl im Modellexperiment als auch im Originalmaßstab übertragen.

Die Stokes'sche Reibungskraft ist vernachlässigbar klein und die Basset-Kraft macht nur circa 5-10\% im Vergleich zur zusätzliche-Masse-Kraft aus. Allerdings hat die Kraftwaage selbst schon einen systematischen Fehler von in etwa 10\%, der sowohl die Amplitude als auch die Phase beeinflusst. 
Genau wie bei $\mathrm{OH}$ konnte gezeigt werden, dass der Gewichtungsfaktor $C_{A}^{*}$ für die zusätzliche-Masse-Kraft, welcher in potentialtheoretischen Strömungen $C_{A}^{*}=2$ ist, auch für größere Reynoldszahlen von diesen unabhängig ist. $C_{A}^{*}$ hängt von der so genannten Beschleunigungszahl Ac ab, welche als das Verhältnis des Hubes, also der Auslenkung der Luft, zum Durchmesser der verwendeten Kugel ausgedrückt werden kann. Die Messungen mit der Kraftwaage haben eine deutliche Frequenzabhängigkeit von $C_{A}^{*}$ gezeigt, was vermuten ließ, dass die Resonanz der Kraftwaage bereits einen großen Einfluss auf die Messung hat.

Daher wurden die Kraftmessungen im Lautsprecherfeld mit mittels optischer Objektverfolgung wiederholt. Diese bestätigten die Unabhängigkeit von $C_{A}^{*}$ von der Reynoldszahl und eine Abhängigkeit von der Beschleunigungszahl $A c . C_{A}^{*}$ nähert sich bereits für kleine $A c$ einem Grenzwert an, so dass vereinfacht $C_{A}^{*}$ gleich dem Grenzwert gesetzt werden kann. Es wurde abgeschätzt, dass die auftretenden Beschleunigungszahlen bei der Zugvorbeifahrt sowohl im 1:25 Modell als auch im Originalmaßstab den verwendeten Beschleunigungszahlen aus dem Lautsprecherexperiment entsprechen, die erhaltenen experimentellen Ergebnisse also auf die Kräfte auf Kugeln bei der Zugvorbeifahrt übertragen werden können.

Ein Vergleich der Messergebnisse aus den TSG-Messungen mit den theoretischen Kräften sowohl mit $C_{A}^{*}=2$, also dem potentialtheoretischen Wert und $C_{A}^{*}=2,3$ zeigen, dass die Abweichung zwischen gemessener und theoretischer Kraftamplitude signifikant reduziert werden kann.

Für Kugeln wurde in dieser Arbeit ein einfaches analytisches Vorhersagemodell für wirkende Kräfte bei der Zugvorbeifahrt entwickelt, welches sowohl für die Kräfte im Modellexperiment, als auch im Originalmaßstab angewendet werden kann, da es unabhängig von der Reynoldszahl ist und nur vom Kugelradius und der durch den Zug verdrängten Luft abhängt.

Die Grenzen des analytischen Modells zeigen sich bei den Kräften auf Scheiben bei der Zugvorbeifahrt. Hier zeigte sich keine Übereinstimmung mit der Potentialtheorie. Die gemessene Kraft auf die Scheibe war mehr als doppelt so hoch wie von der Potentialtheorie vorhergesagt. Ein Vergleich mit der stationären Aerodynamik zeigte ebenfalls keine Übereinstimmung. Lediglich eine Kombination und Gewichtung der instationär und stationär berechneten Kraft konnte die gemessenen Daten abbilden. Bei der Scheibe löst die Strömung an den scharfen Kanten ab, so dass die Potentialtheorie als alleinige Beschreibung unzureichend ist. Allerdings genügt auch die stationäre Aerodynamik nicht. Dieser Zusammenhang und der physikalische Hintergrund der Kräfte auf Scheiben als weitere Körperformen sollte in zukünftigen Arbeiten weiter untersucht werden. 



\section{Literaturverzeichnis}

[1] Bechert, D: Der Einfluss der aerodynamischen Druckwelle auf die Geräuschmessung bei Hochgeschwindigkeitszügen / DFVLR Institut für Exprimentelle Strömungsmechanik Göttingen, Abteilung Turbulenzforschung. 1980. - Forschungsbericht

[2] Ehrenfried, K.: Messungen zur Zug-Infrastruktur-Wechselwirkung in der Tunnelsimulationsanlage TSG / Deutsches Zentrum für Luft- und Raumfahrt. 2013. Forschungsbericht

[3] Stokes, G. G.: Mathematical and Physical Papers. 3 (1851), Nr. 1

[4] Basset, A. B.: A treatise on hydrodynamics: with numerous examples. Bd. 2. Deighton, Bell and Company, 1888

[5] Boussinesq, J. V.: Sur la resistance ... d'une sphere solide. In: C.R. des Seances de l'Academie 100 (1885)

[6] Oseen, C. W.: Hydrodynamik. Akademische Verlagsgesellschaft, 1927

[7] Mei, R ; Lawrence, C. J. ; Adrian, R. J.: Unsteady drag on a sphere at finite Reynolds number with small fluctuations in the free stream velocity. In: Journal of Fluid Mechanics 233 (1991), S. 613-631

[8] MeI, R ; Adrian, R. J.: Flow past a sphere with an oscillation in the free-strem velocity and unsteady drag at finite Reynolds number. In: Journal of Fluid Mechanics 237 (1992), S. 323-341

[9] Lovalenti, P. M. ; Brady, J. F.: The force on a sphere in a uniform flow with small-amplitude oscillations at finite Reynolds number. In: Journal of Fluid Mechanics 256 (1993), S. 607-614 


\section{LITERATURVERZEICHNIS}

[10] MeI, R: Flow due to an oscillating sphere and an expression for unsteady drag on the sphere at finite Reynolds number. In: Journal of Fluid Mechanics 270 (1994), S. $133-174$

[11] Odar, F. ; Hamilton, W. S.: Forces on a sphere accelerating in a viscous fluid. In: Journal of Fluid Mechanics 18 (1964), Nr. 2, S. 302-314

[12] Steinheuer, J.: Aerodynamische Wirkungen von schnellfahrenden Schienenfahrzeugen auf die Umgebung / Institut für Entwurfsaerodynamik Braunschweig. 1981.

- Forschungsbericht

[13] Amtsblatt der Europäischen Union: Entscheidung der Kommission vom 21. Februar 2008 Über die technische Spezifikation für die Interoperabilität des Teilsystems Fahrzeuge des transeuropäischen Hochgeschwindigkeitsbahnsystems. 32008

[14] Mackrodt, P.-A. ; Pfizenmaier, E.: Aerodynamik und Aeroakustik für Hochgeschwindigkeitszüge. In: Physik in unserer Zeit 18 (1987), Nr. 3, 65-76. http: //dx.doi.org/10.1002/piuz.19870180301. - ISSN 1521-3943

[15] Baker, C: The flow around high speed trains / School of Civil Engineering University of Birmingham, Edgbaston, Birmingham, B15 2TT, United Kingdon. 2008. - Forschungsbericht

[16] Hucho, W.-H.: Aerodynamik der stumpfen Körper. Vieweg Teubner Verlag, 2011

[17] Jordan, S.C. ; Sterling, M. ; Baker, C.J.: Modelling the response of a standing person to the slipstream generated by a passenger train. In: Proceedings of the Institution of Mechanical Engineers, Part F: Journal of Rail and Rapid Transit 223 (2009), S. 567-579

[18] Gerhardt, H.J ; Krüger, O: Wind and train driven air movements in train stations. In: Journal of Wind Engineering and Industrial Aerodynamics 74-76 (1998), Nr. 0, 589 - 597. http://www.sciencedirect.com/science/article/pii/ S0167610598000531. - ISSN 0167-6105

[19] Sanz-Andres, A ; Santiago-Prowald, J: Train-induced pressure on pedestrians. In: Journal of Wind Engineering and Industrial Aerodynamics 90 (2002), Nr. 8, 1007 - 1015. http://www.sciencedirect.com/science/article/pii/ S0167610502002167. - ISSN 0167-6105

[20] Sanz-Andres, A. ; A.Laver'on ; Cuerva, A. ; Baker, C.: Vehicle-induced force on pedestrians. In: Journal of Wind Engineering and Industrial Aerodynamics 92 (2004), S. 185-198

[21] Wieghardt, K. ; Dillmann, A. (Hrsg.): Theoretische Strömungsmechanik. Universitätsverlag Göttingen, 2005 
[22] Frenzel, B. ; Gebhard, F.: Physik Formelsammlung: FüIngenieure und Naturwissenschaftler. Springer Science \& Business Media, 2007

[23] Collatz, G.: Potentialtheoretische Untersuchungen der hydrodynamischen Wechselwirkung zweier Schiffskörper. In: Jahrbuch d. Schiffbautechnischen Gesellschaft. Bd 57. Berlin, Göttingen, Heidelberg, Springer Verlag, 1963, S. 281-329

[24] Butcher, J. C.: Numerical Methods for Ordinary Differential Equations. John Wiley \& Sons, New York, ISBN 978-0-471-96758-3., 2003

[25] Ehrenfried, K.: Strömungsakustik: Skript zur Vorlesung. Mensch \& Buch, 2004

[26] Lord RAYLEIGH F.R.S.: XXXVII. On the passage of waves through apertures in plane screens, and allied problems. In: Philosophical Magazine Series 5 43:263 (1897), S. 259-272

[27] Sir Horace Lamb: Hydrodynamics. Cambridge University Press, 1895

[28] Möser, M.: Körperschall. Springer, 1967

[29] Sanz-Andres, A. ; Santiago-Prowald, J. ; Baker, C. ; Quinn, A.: Vehicleinduced loads on traffic sign panels. In: Journal of Wind Engineering and Industrial Aerodynamics 91 (2003), Nr. 7, 925 - 942. http://www. sciencedirect.com/ science/article/pii/S0167610503000357. - ISSN 0167-6105

[30] Johnson, T ; Dalley, S: 1:25 Scale Moving Model Tests for the TRANSAERO Project. In: A eurpopean initiative on transient aerodynamics for railway system optimisation, Springer Berlin Heidelberg, 2002, S. 123-135

[31] Loose, S. ; Heckmann, A.: Contributions of aerodynamic. In: Europe Rail Technology Review Special: NGT- Next Generation Train (2011), S. 41-50. - TZ-Verlag \& Print GmbH, ISBN 978-3-7771-0435-5

[32] Dantec Dynamics: StreamLine Pro Anemometer System. Publication No.: PI119v7. http://www.dantecdynamics.com/docs/products-and-services/ fluid-mechanics/cta/PI119_StreamLine_Pro_Anemometer_System.pdf. - Abgerufen am 28.11.2016

[33] Bruun, H. H.: Hot-wire Anemometry: Principles and Signal Analysis. Oxford University Press, 1995

[34] TSI: TSI THERMAL ANEMOMETRY PROBES. http://www.tsi.com/ uploadedFiles/_Site_Root/Products/Literature/Catalogs/Hotwire_ Catalog_2980465.pdf. Version: 2013. - Abgerufen am 28.11.2016

[35] KIng, L. S.: On the Convection Heat Transfer from Small Cylinders in a Stream of Fluids. Determination of Convection Constants of Small Platinum Wires with Application to Hot-Wire Anemometry. In: Phil. Trans. Roy. Soc. London 214A (1914), S. $373-432$ 


\section{LITERATURVERZEICHNIS}

[36] METEK: Phantom v1210 Data Sheet. http://www.ptnordic.dk/media/30427/ phantom_v1610_-v1210.pdf. Version: 2013. - Abgerufen am 28.11.2016

[37] NikON: UV-Nikkor $105 \mathrm{~mm}$ f/4.5 Instruction Manual

[38] Canny, J: A Computational Approach to Edge Detection. In: IEEE Trans Pattern Anal Mach Intell 8(6) (1986), S. 679-698

[39] Rutschmann, S.: Experimentelle Untersuchungen zur Anwendbarkeit einer Kraftmesstechnik basierend auf optischer Objektverfolgung in CCD-Kameraaufnahmen. Bachelorarbeit am Insitut für Aerodynamik und Strömungstechnik am Deutschen Zentrum für Luft- und Raumfahrt Göttingen und Georg-August-Universität Göttingen, 2009

[40] Sobel, I. E.: Camera Models and Machine Perception, Stanford University, Diss., 1970

[41] In: Moré, J. J.: The Levenberg-Marquardt Algorithm: Implementation and Theory. Springer, Berlin, Heidelberg, New York, 1978, S. 105-116

[42] Savitzky, A. ; Golay, M. J. E.: Smoothing and Differentiation of Data by Simplified Least Squares Procedures. In: Analytical Chemistry 36 (1964), Nr. 8, S. $1627-1639$

[43] Kreiss, J-P ; Neuhaus, G.: Einführung in die Zeitreihenanalyse. Springer, 2006

[44] Lapple, C. E.: Particle Dynamics. In: Ver. Deut. Ing (1951)

[45] RCF: Woofer LF18N401 Data Sheet. http://www.rcf.it/de_DE/products/ precision-transducers/neodymium-low-frequency-transducers/LF18N401. abgerufen am 23.12.2016

[46] Polytec: OFV-5000 Vibrometer Controller Datenblatt. http://www . polytec.com/de/produkte/schwingungsmesssysteme/einpunkt-vibrometer/ modulare-systeme/ofv-5000-vibrometer-controller/. Version: 102016. Abgerufen am 14.12.2016

[47] Polytec: OFV-505 Messkopf Datenblatt. http://www.polytec. com/de/produkte/schwingungsmesssysteme/einpunkt-vibrometer/ modulare-systeme/ofv-50x-vibrometer-messkopf/. Version: 102015. Abgerufen am 14.12.2016

[48] Microflown Technologies: Manual PU Mini. http://www.microflown.com/ products/standard-probes/pu-mini.html. - Abgerufen am 23.12.2016

[49] Schiller, L. ; Naumann, A: Über die grundlegende Berechnung bei der Schwerkraftaufbereitung. In: Ver. Deutscher Ingenieure 77 (1933), S. 318-320 
[50] Michaelides, E. E. ; Roig, A.: A Reinterpretation of the Odar and Hamilton Data on the Unsteady Equation of Motion of Particles. In: AIChE Journal 57 (2011), S. 2997-3002

[51] Endevco: Piezoresistive pressure transducer. - Data Sheet

[52] Boltze, E.: Grenzschichten an Rotationskörpern in Flüssigkeiten mit kleiner Reibung, Georg-August-Universität Göttingen, Diss., 1908 

Appendices 

ANHANG $\mathrm{A}$

Geschwindigkeitskurven der Vorbeifahrtmessungen

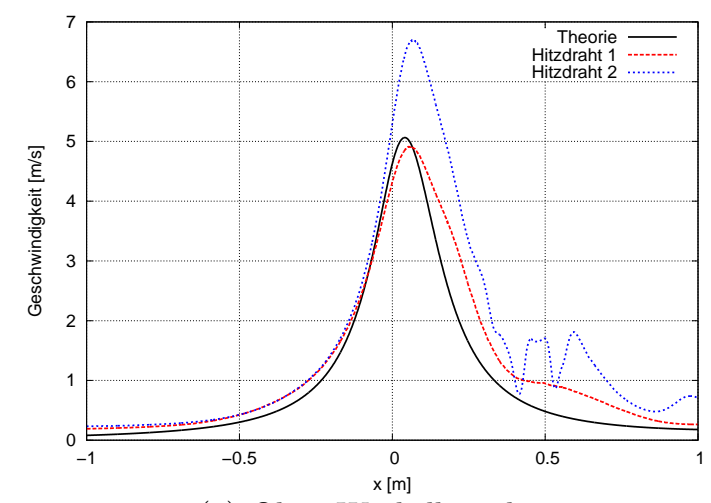

(a) Ohne Winkelkorrektur

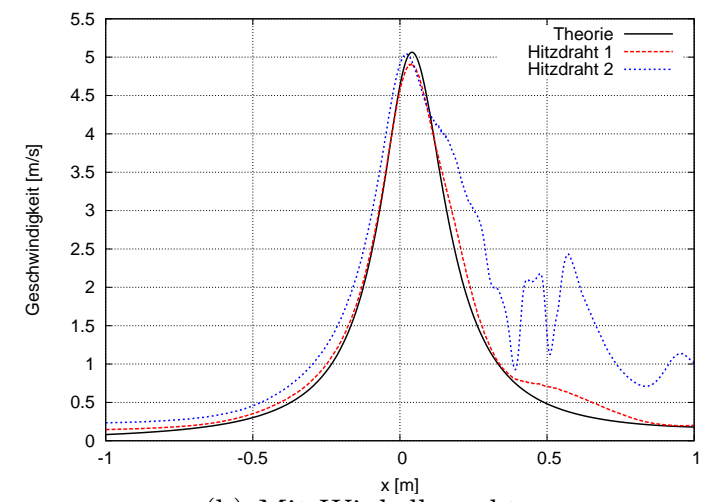

(b) Mit Winkelkorrektur

Abbildung A.1.: Vergleich unkorrigiertes und korrigiertes Geschwindigkeitssignal für $y_{M}=10 \mathrm{~cm}, z_{M}=4,5 \mathrm{~cm}, u_{\infty}=40,48 \mathrm{~m} / \mathrm{s}$. 


\section{A. Geschwindigkeitskurven der Vorbeifahrtmessungen}

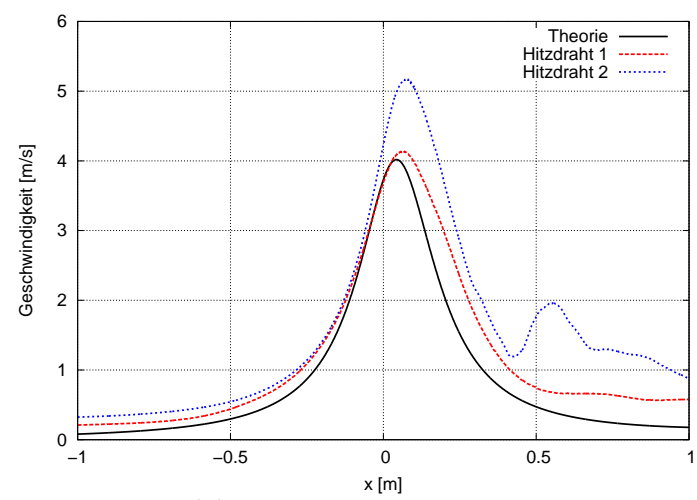

(a) Ohne Winkelkorrektur

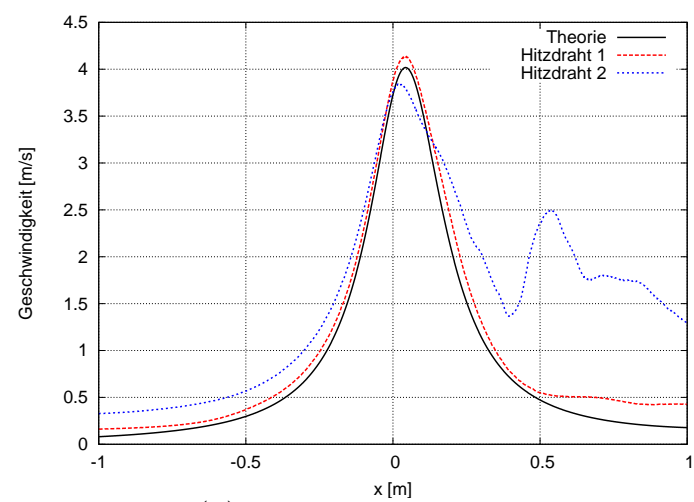

(b) Mit Winkelkorrektur

Abbildung A.2.: Vergleich unkorrigiertes und korrigiertes Geschwindigkeitssignal für $y_{M}=12 \mathrm{~cm}, z_{M}=4,5 \mathrm{~cm}, u_{\infty}=40,27 \mathrm{~m} / \mathrm{s}$.

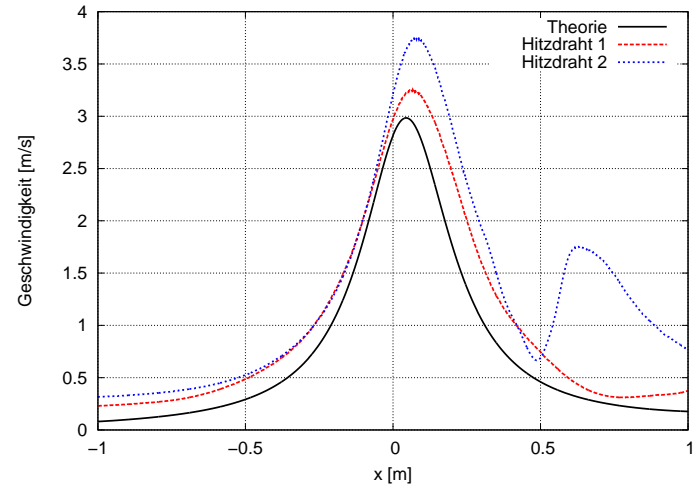

(a) Ohne Winkelkorrektur

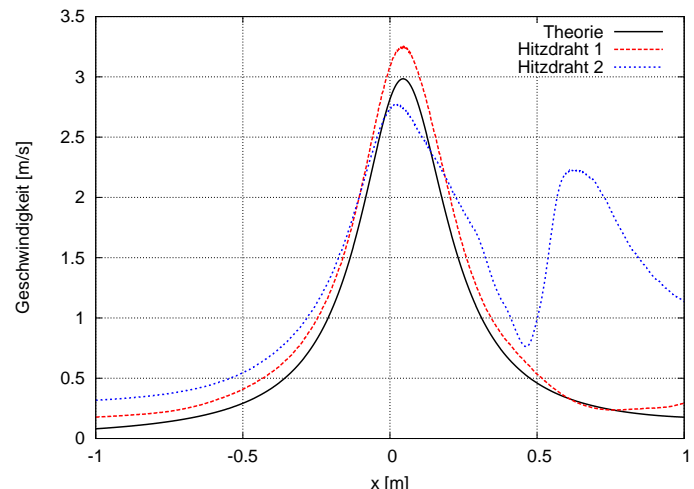

(b) Mit Winkelkorrektur

Abbildung A.3.: Vergleich unkorrigiertes und korrigiertes Geschwindigkeitssignal für $y_{M}=15 \mathrm{~cm}, z_{M}=4,5 \mathrm{~cm}, u_{\infty}=40,24 \mathrm{~m} / \mathrm{s}$. 


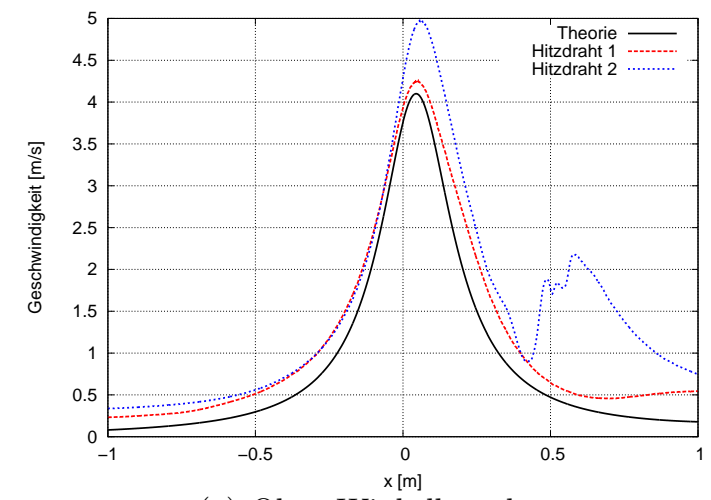

(a) Ohne Winkelkorrektur

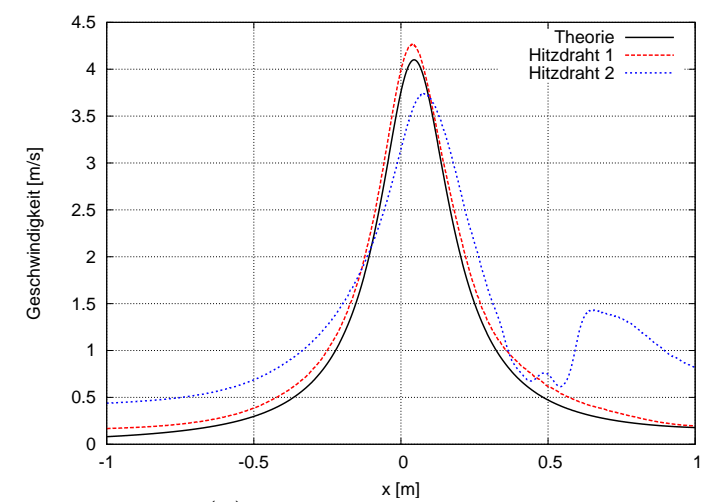

(b) Mit Winkelkorrektur

Abbildung A.4.: Vergleich unkorrigiertes und korrigiertes Geschwindigkeitssignal für $y_{M}=12 \mathrm{~cm}, z_{M}=6 \mathrm{~cm}, u_{\infty}=40,27 \mathrm{~m} / \mathrm{s}$.

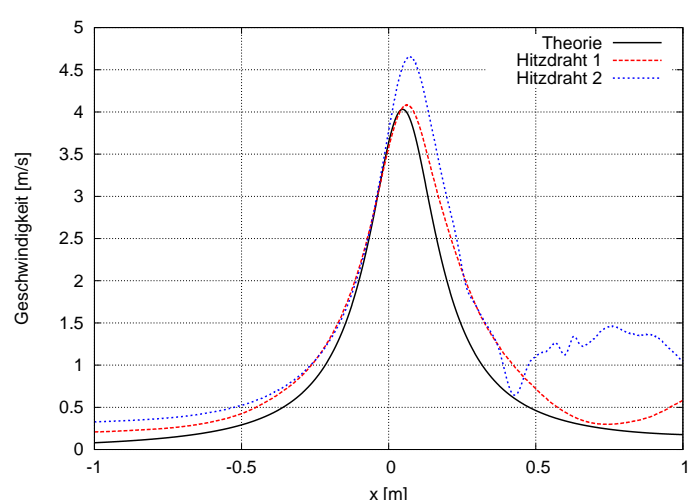

(a) Ohne Winkelkorrektur

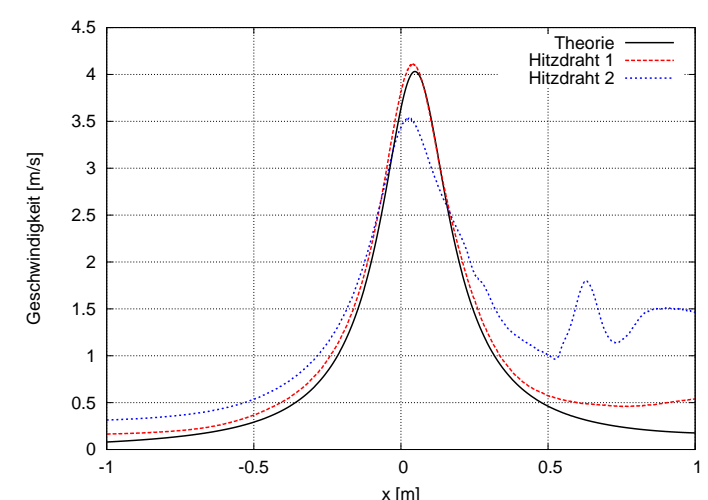

(b) Mit Winkelkorrektur

Abbildung A.5.: Vergleich unkorrigiertes und korrigiertes Geschwindigkeitssignal für $y_{M}=12 \mathrm{~cm}, z_{M}=8 \mathrm{~cm}, u_{\infty}=40,15 \mathrm{~m} / \mathrm{s}$. 
A. Geschwindigkeitskurven der Vorbeifahrtmessungen

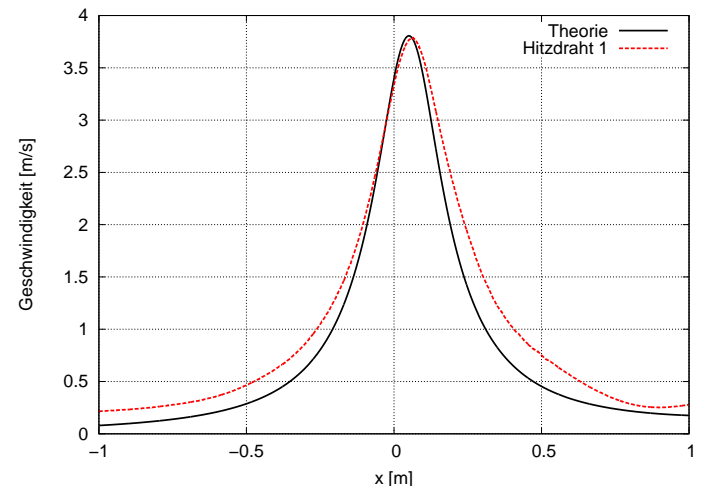

(a) Ohne Winkelkorrektur

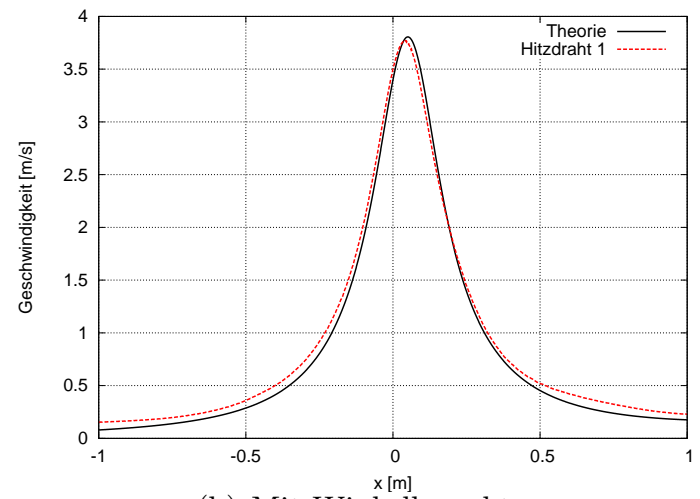

(b) Mit Winkelkorrektur

Abbildung A.6.: Vergleich unkorrigiertes und korrigiertes Geschwindigkeitssignal für $y_{M}=12 \mathrm{~cm}, z_{M}=10 \mathrm{~cm}, u_{\infty}=40,33 \mathrm{~m} / \mathrm{s}$. Hitzdraht 2 ist bei diesem Versuch leider kaputt gegangen. 


\section{ANHANG B}

\section{Kraftkurven der Vorbeifahrtmessungen}

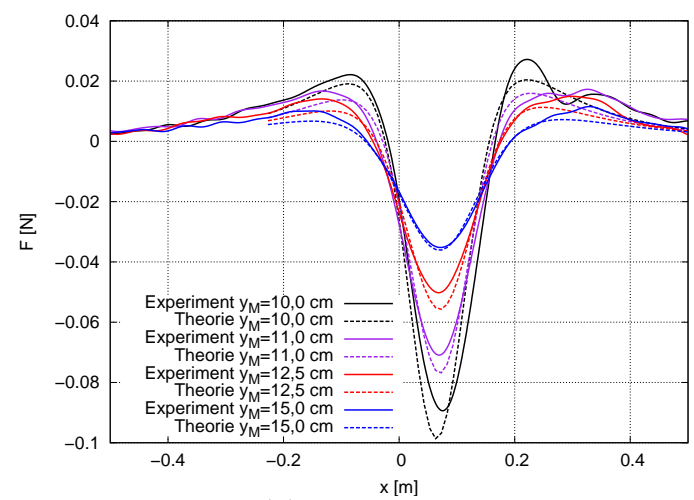

(a) x-Richtung

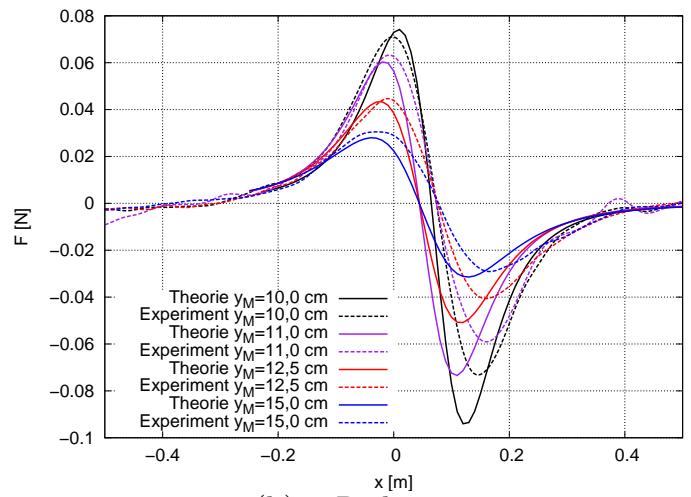

(b) y-Richtung

Abbildung B.1.: Vergleich der experimentellen Kräfte induziert vom ICE3-Modell auf die Kugel $(R=2 \mathrm{~cm})$ für verschiedene Gleisabstände mit den jeweiligen potentialtheoretischen Kraftkurven für $z_{M}=4,5 \mathrm{~cm}$ und $u_{\infty}=37,7 \mathrm{~m} / \mathrm{s}$. 


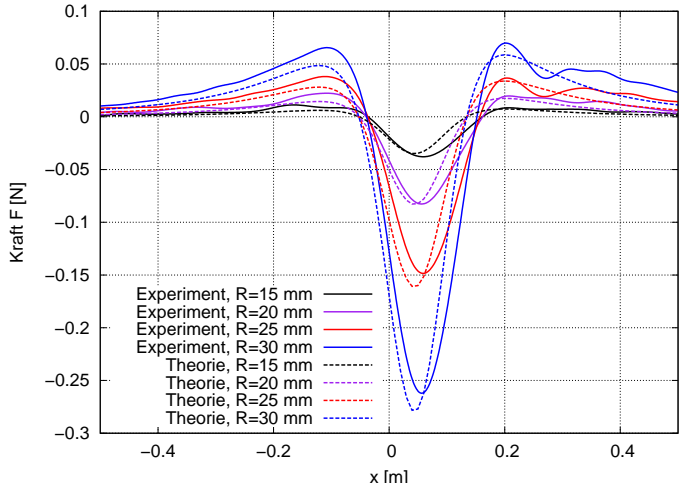

(a) x-Richtung

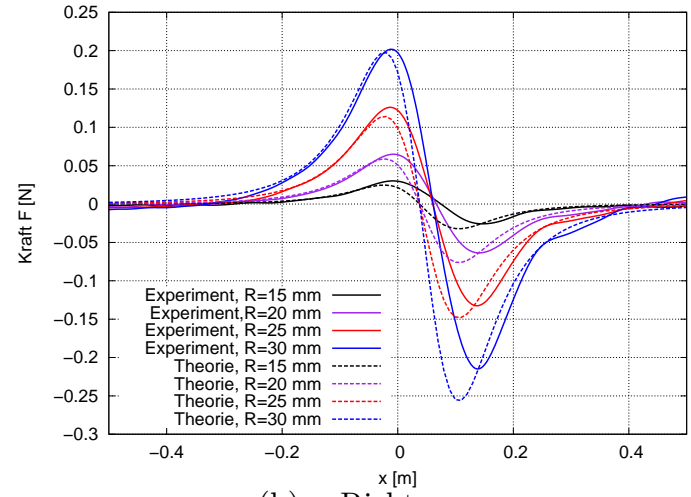

(b) y-Richtung

Abbildung B.2.: Vergleich der experimentellen Kräfte induziert vom ICE3-Modell auf verschiedene Kugeln mit den jeweiligen potentialtheoretischen Kraftkurven für $z_{M}=4,5 \mathrm{~cm}, u_{\infty}=37,7 \mathrm{~m} / \mathrm{s}$ und $y_{M}=11 \mathrm{~cm}$.

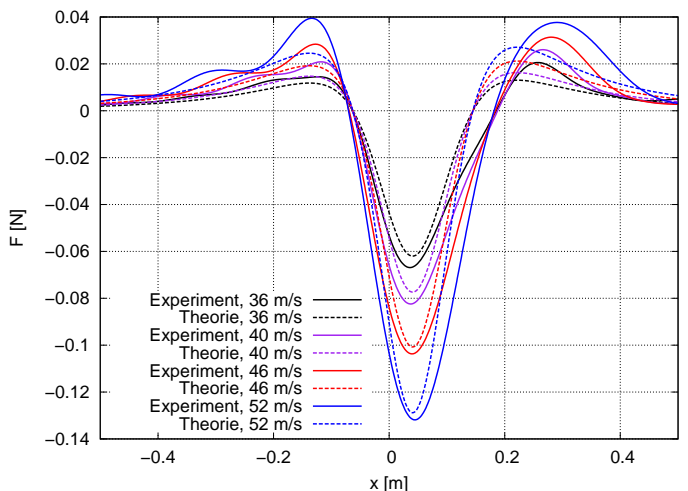

(a) x-Richtung

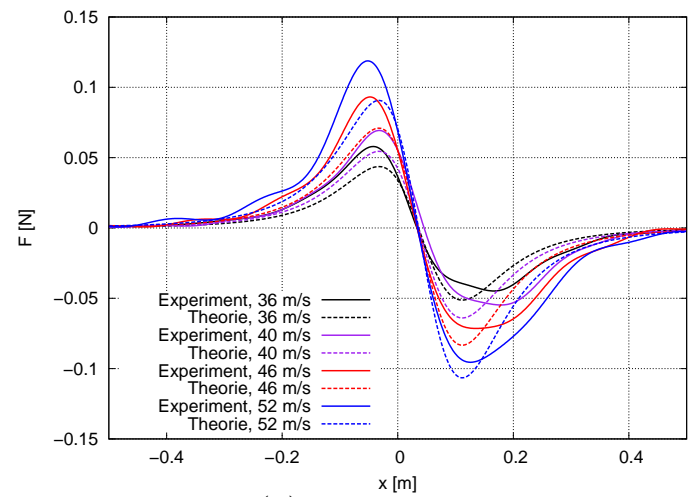

(b) y-Richtung

Abbildung B.3.: Vergleich der experimentellen Kräfte auf die Kugel $(R=2 \mathrm{~cm})$ induziert vom Potentialmodell für verschiedene Fahrtgeschwindigkeiten mit den jeweiligen potentialtheoretischen Kraftkurven für $z_{M}=4,5 \mathrm{~cm}$ und $y_{M}=12 \mathrm{~cm}$. 


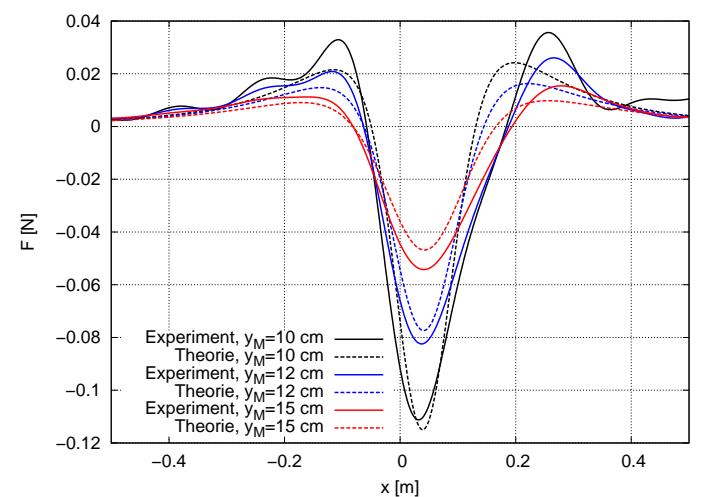

(a) x-Richtung

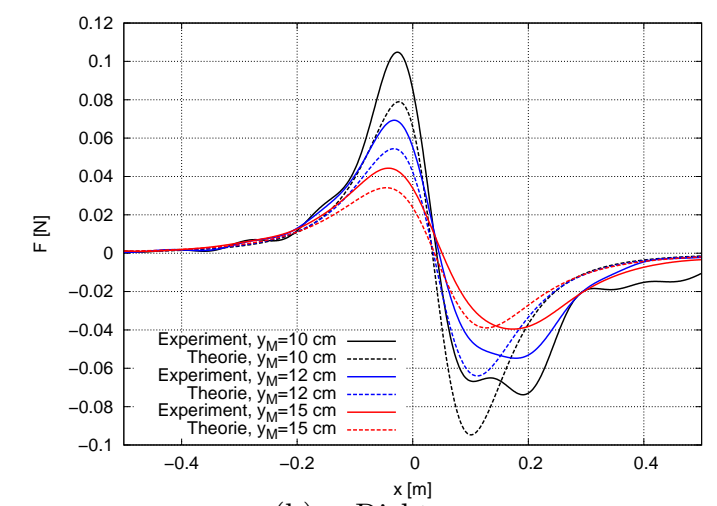

(b) y-Richtung

Abbildung B.4.: Vergleich der experimentellen Kräfte auf die Kugel $(R=2 \mathrm{~cm})$ induziert vom Potentialmodell für verschiedene Gleisabstände mit den jeweiligen potentialtheoretischen Kraftkurven für $z_{M}=4,5 \mathrm{~cm}$ und $u_{\infty}=40 \mathrm{~m} / \mathrm{s}$.

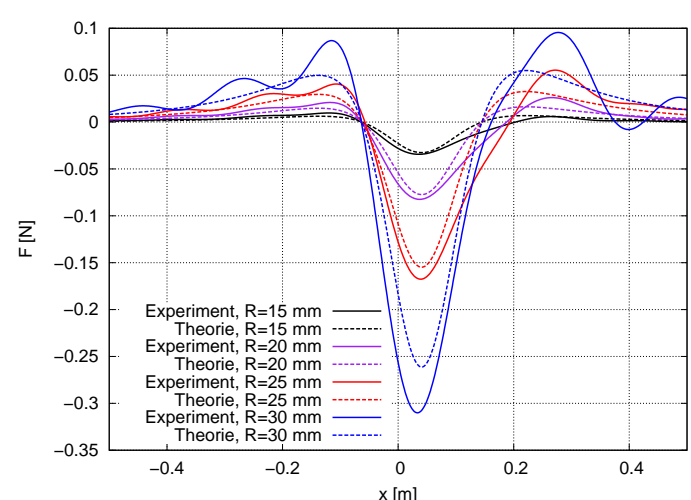

(a) x-Richtung

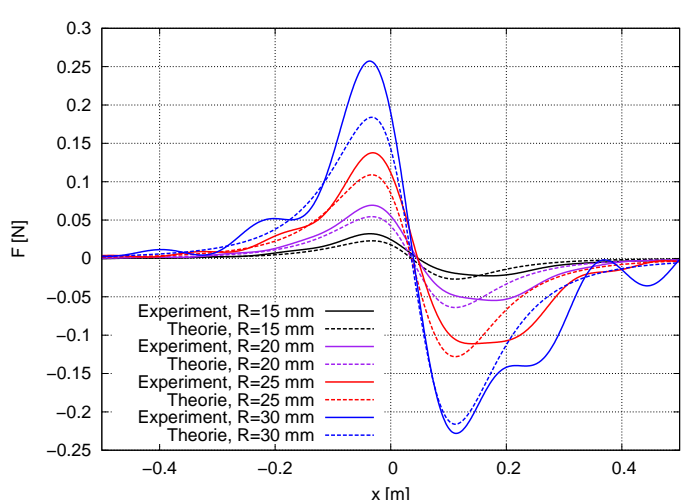

(b) y-Richtung

Abbildung B.5.: Vergleich der experimentellen Kräfte induziert vom Potentialmodell auf verschiedene Kugeln mit den jeweiligen potentialtheoretischen Kraftkurven für $z_{M}=4,5 \mathrm{~cm}, u_{\infty}=40 \mathrm{~m} / \mathrm{s}$ und $y_{M}=12 \mathrm{~cm}$. 


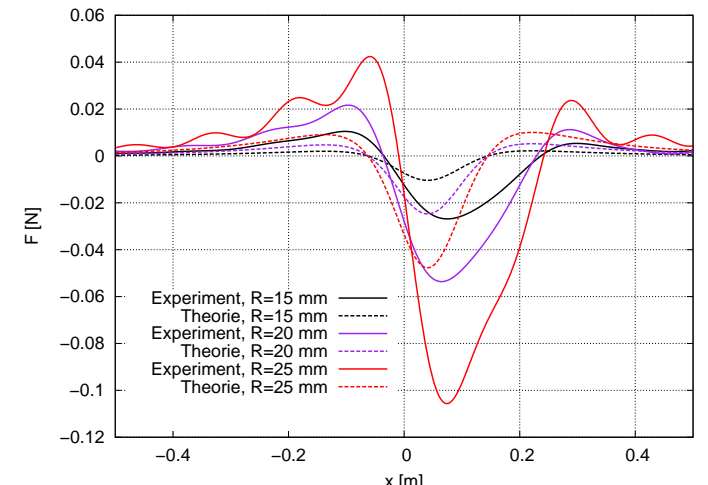

(a) x-Richtung

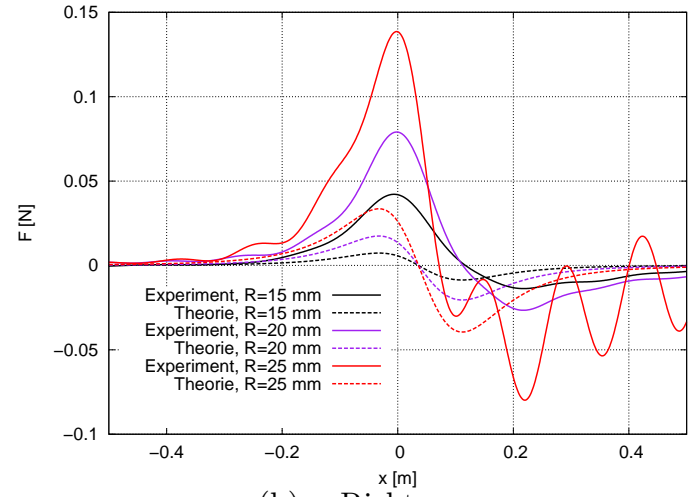

(b) y-Richtung

Abbildung B.6.: Vergleich der experimentellen Kräfte induziert vom Potentialmodell auf verschiedene Scheiben mit den jeweiligen potentialtheoretischen Kraftkurven für $z_{M}=4,5 \mathrm{~cm}, u_{\infty}=40 \mathrm{~m} / \mathrm{s}$ und $y_{M}=12 \mathrm{~cm}$.

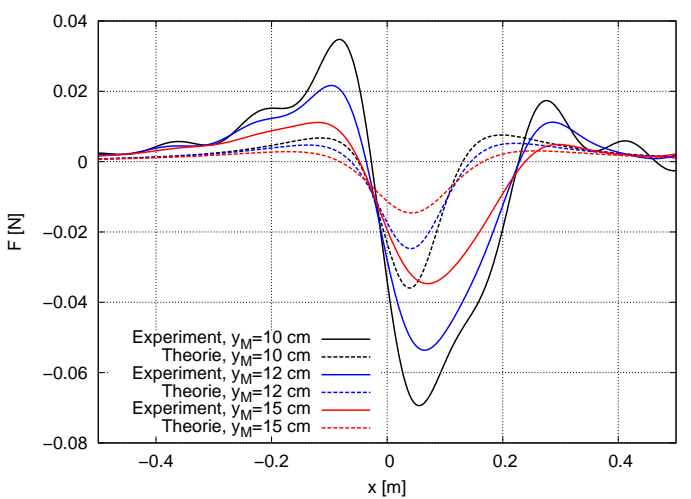

(a) x-Richtung

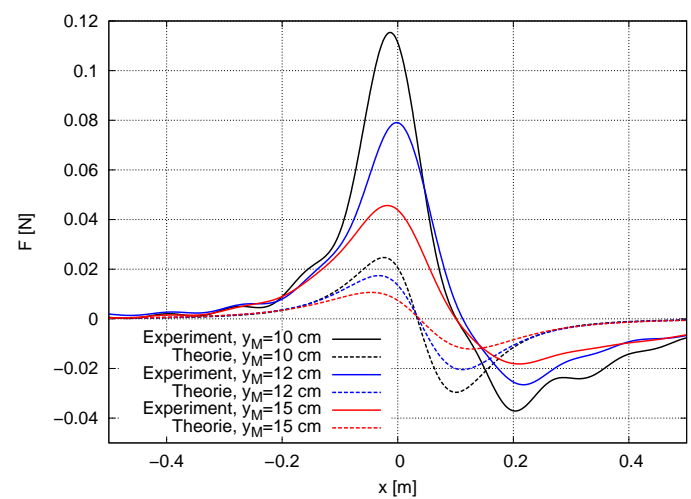

(b) y-Richtung

Abbildung B.7.: Vergleich der experimentellen Kräfte induziert vom Potentialmodell auf die Scheibe $(R=2 \mathrm{~cm})$ für verschiedene Gleisabstände mit den jeweiligen potentialtheoretischen Kraftkurven für $z_{M}=4,5 \mathrm{~cm}$ und $u_{\infty}=40 \mathrm{~m} / \mathrm{s}$. 
anhang $\mathrm{C}$

Lautsprechermessungen

\begin{tabular}{|c|c|} 
Frequenz $\mathrm{f}[\mathrm{Hz}]$ & Faktor $(\mathrm{f})=v_{\text {Kugelhöhe }} / v_{\text {Membran }}$ \\
\hline \hline 10 & 0,491 \\
\hline 14 & 0,492 \\
\hline 18 & 0,501 \\
\hline 20 & 0,502 \\
\hline 24 & 0,508 \\
\hline 28 & 0,513 \\
\hline 30 & 0,514 \\
\hline 34 & 0,520 \\
\hline 38 & 0,518 \\
\hline 41 & 0,524 \\
\hline 60 & 0,558 \\
\hline 71 & 0,560 \\
\hline 81 & 0,543 \\
\hline 91 & 0,549 \\
\hline 101 & 0,546 \\
\hline 110 & 0,538 \\
\hline 131 & 0,537 \\
\hline 151 & 0,527 \\
\hline 171 & 0,515 \\
\hline 191 & 0,501 \\
\hline
\end{tabular}

Tabelle C.1.: Normierfaktoren für die Geschwindigkeit auf Kugelhöhe. 


\begin{tabular}{|c|c|c|c|c|c|c|c|c|}
\hline $\mathrm{f}[\mathrm{Hz}]$ & Amp $[\mathrm{mV}]$ & $\mathrm{R}[\mathrm{mm}]$ & $\operatorname{Re}$ & $\mathrm{CA}$ & CD & $\mathrm{CH}$ & $\mathrm{A} 0 / \mathrm{R}$ & $F_{H} / F_{A}[\%]$ \\
\hline 30 & 100 & 20 & 343,47 & 1,89 & 0,65 & 12,30 & 0,034 & 9,21 \\
\hline 30 & 100 & 25 & 429,79 & 1,86 & 0,60 & 10,77 & 0,028 & 6,56 \\
\hline 30 & 100 & 30 & 512,81 & 1,84 & 0,56 & 10,11 & 0,023 & 5,18 \\
\hline 30 & 150 & 20 & 519,52 & 1,89 & 0,55 & 11,77 & 0,052 & 8,80 \\
\hline 30 & 150 & 25 & 649,37 & 1,86 & 0,51 & 10,70 & 0,042 & 6,51 \\
\hline 30 & 150 & 30 & 775,25 & 1,84 & 0,48 & 10,39 & 0,034 & 5,33 \\
\hline 30 & 200 & 20 & 696,81 & 1,88 & 0,50 & 12,69 & 0,070 & 9,52 \\
\hline 30 & 200 & 25 & 870,83 & 1,86 & 0,46 & 11,00 & 0,056 & 6,71 \\
\hline 30 & 200 & 30 & 1040,15 & 1,83 & 0,43 & 11,11 & 0,046 & 5,71 \\
\hline 30 & 300 & 15 & 789,77 & 1,97 & 0,48 & 16,52 & 0,140 & 15,81 \\
\hline 30 & 300 & 20 & 1051,99 & 1,87 & 0,43 & 13,32 & 0,105 & 10,05 \\
\hline 30 & 300 & 25 & 1313,72 & 1,85 & 0,40 & 11,87 & 0,084 & 7,26 \\
\hline 30 & 300 & 30 & 1570,19 & 1,83 & 0,38 & 11,68 & 0,070 & 6,01 \\
\hline 30 & 350 & 15 & 923,73 & 1,96 & 0,45 & 17,56 & 0,164 & 16,92 \\
\hline 30 & 350 & 20 & 1230,39 & 1,88 & 0,41 & 13,75 & 0,123 & 10,37 \\
\hline 30 & 350 & 25 & 1535,31 & 1,85 & 0,38 & 11,88 & 0,098 & 7,26 \\
\hline 30 & 350 & 30 & 1837,37 & 1,83 & 0,36 & 12,26 & 0,082 & 6,33 \\
\hline 30 & 400 & 15 & 1057,62 & 1,97 & 0,43 & 17,59 & 0,188 & 16,85 \\
\hline 30 & 400 & 20 & 1408,97 & 1,87 & 0,39 & 14,38 & 0,141 & 10,87 \\
\hline 30 & 400 & 25 & 1758,12 & 1,84 & 0,36 & 12,42 & 0,112 & 7,61 \\
\hline 30 & 400 & 30 & 2103,72 & 1,82 & 0,34 & 12,70 & 0,093 & 6,56 \\
\hline 71 & 100 & 15 & 263,95 & 2,39 & 0,72 & 1,16 & 0,020 & 0,59 \\
\hline 71 & 100 & 20 & 351,47 & 2,13 & 0,64 & 5,91 & 0,015 & 2,55 \\
\hline 71 & 100 & 25 & 439,63 & 2,06 & 0,59 & 9,07 & 0,012 & 3,23 \\
\hline 71 & 100 & 30 & 528,58 & 2,07 & 0,55 & 14,17 & 0,010 & 4,20 \\
\hline 71 & 150 & 15 & 394,74 & 2,38 & 0,61 & 1,95 & 0,030 & 1,00 \\
\hline 71 & 150 & 20 & 525,61 & 2,13 & 0,55 & 6,01 & 0,022 & 2,59 \\
\hline 71 & 150 & 25 & 658,11 & 2,06 & 0,51 & 9,21 & 0,018 & 3,28 \\
\hline 71 & 150 & 30 & 791,21 & 2,07 & 0,48 & 13,52 & 0,015 & 4,00 \\
\hline 71 & 200 & 15 & 524,82 & 2,38 & 0,55 & 3,03 & 0,039 & 1,56 \\
\hline 71 & 200 & 20 & 699,10 & 2,13 & 0,50 & 6,76 & 0,030 & 2,92 \\
\hline 71 & 200 & 25 & 875,30 & 2,06 & 0,46 & 9,29 & 0,024 & 3,31 \\
\hline 71 & 200 & 30 & 1052,25 & 2,07 & 0,43 & 13,71 & 0,020 & 4,05 \\
\hline 110 & 100 & 15 & 193,82 & 2,54 & 0,82 & 17,94 & 0,009 & 6,96 \\
\hline 110 & 100 & 20 & 258,26 & 2,34 & 0,73 & 20,58 & 0,007 & 6,50 \\
\hline 110 & 100 & 25 & 322,88 & 2,34 & 0,66 & 25,27 & 0,006 & 6,38 \\
\hline 110 & 100 & 30 & 388,59 & 2,51 & 0,62 & 36,91 & 0,005 & 7,23 \\
\hline 110 & 150 & 15 & 289,27 & 2,54 & 0,69 & 16,90 & 0,014 & 6,56 \\
\hline 110 & 150 & 20 & 385,88 & 2,33 & 0,62 & 21,09 & 0,011 & 6,69 \\
\hline 110 & 150 & 25 & 482,29 & 2,34 & 0,57 & 25,41 & 0,008 & 6,41 \\
\hline
\end{tabular}




\begin{tabular}{|r|r|r|r|r|r|r|r|r|}
\hline 110 & 150 & 30 & 580,54 & 2,51 & 0,53 & 36,90 & 0,007 & 7,24 \\
\hline 110 & 200 & 15 & 382,12 & 2,55 & 0,62 & 16,26 & 0,019 & 6,29 \\
\hline 110 & 200 & 20 & 509,70 & 2,33 & 0,56 & 20,67 & 0,014 & 6,54 \\
\hline 110 & 200 & 25 & 637,95 & 2,35 & 0,51 & 25,23 & 0,011 & 6,36 \\
\hline 110 & 200 & 30 & 765,51 & 2,52 & 0,48 & 36,65 & 0,009 & 7,16 \\
\hline 110 & 300 & 15 & 560,45 & 2,55 & 0,54 & 15,97 & 0,027 & 6,16 \\
\hline 110 & 300 & 20 & 747,52 & 2,34 & 0,49 & 20,58 & 0,020 & 6,51 \\
\hline 110 & 300 & 25 & 937,39 & 2,35 & 0,45 & 25,57 & 0,016 & 6,44 \\
\hline 110 & 300 & 30 & 1127,23 & 2,51 & 0,42 & 37,68 & 0,014 & 7,39 \\
\hline 131 & 100 & 15 & 152,17 & 2,71 & 0,90 & 14,84 & 0,006 & 4,94 \\
\hline 131 & 100 & 20 & 205,06 & 2,48 & 0,80 & 34,58 & 0,005 & 9,42 \\
\hline 131 & 100 & 25 & 253,93 & 2,56 & 0,73 & 52,70 & 0,004 & 11,16 \\
\hline 131 & 100 & 30 & 305,91 & 2,87 & 0,68 & 89,71 & 0,003 & 14,12 \\
\hline 131 & 150 & 15 & 227,25 & 2,72 & 0,76 & 12,62 & 0,009 & 4,18 \\
\hline 131 & 150 & 20 & 305,77 & 2,48 & 0,68 & 34,27 & 0,007 & 9,36 \\
\hline 131 & 150 & 25 & 379,57 & 2,55 & 0,62 & 52,27 & 0,006 & 11,09 \\
\hline 131 & 150 & 30 & 457,46 & 2,86 & 0,58 & 88,81 & 0,005 & 13,99 \\
\hline 131 & 200 & 15 & 300,22 & 2,74 & 0,68 & 12,46 & 0,012 & 4,12 \\
\hline 131 & 200 & 20 & 404,88 & 2,48 & 0,61 & 34,11 & 0,009 & 9,30 \\
\hline 131 & 200 & 25 & 502,53 & 2,56 & 0,56 & 51,94 & 0,007 & 11,00 \\
\hline 131 & 200 & 30 & 604,68 & 2,87 & 0,52 & 89,40 & 0,006 & 14,09 \\
\hline
\end{tabular}

Tabelle C.2.: Ergebnisse: Messung mit der Kraftwaage.

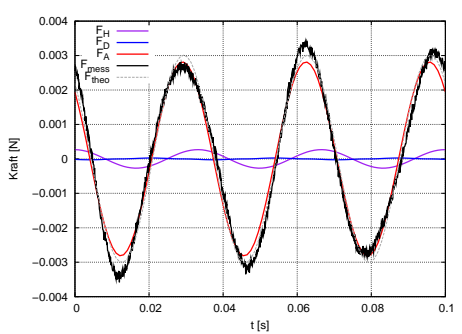

(a) $R=\stackrel{\text { 18 }}{20} \mathrm{~mm}$

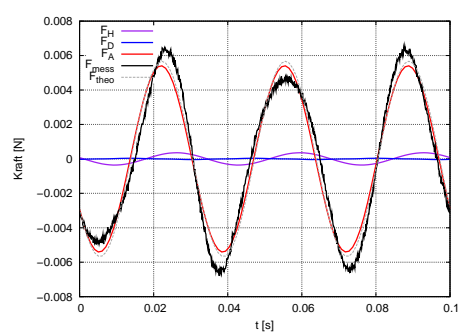

(b) $R=25 \mathrm{~mm}$

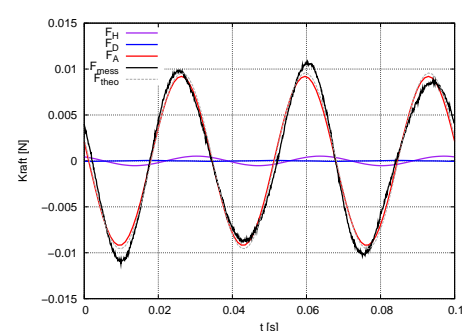

(c) $R=30 \mathrm{~mm}$

Abbildung C.1.: Kraftanteile im Vergleich mit der gemessenen Kraft für $f=30 \mathrm{~Hz}$. 


\section{Lautsprechermessungen}

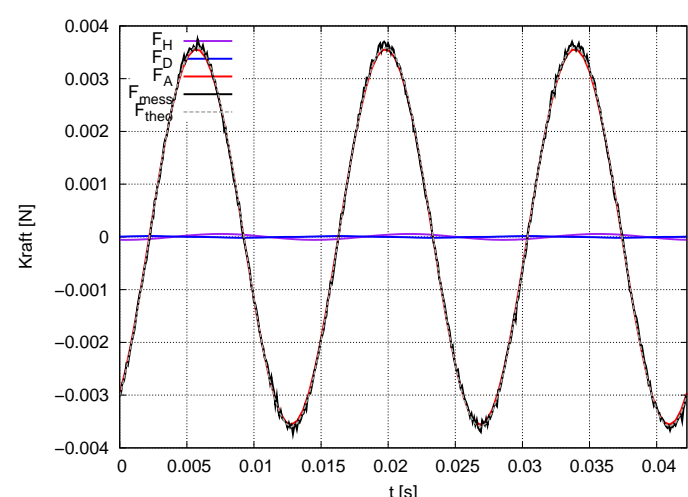

(a) $R=15 \mathrm{~mm}$

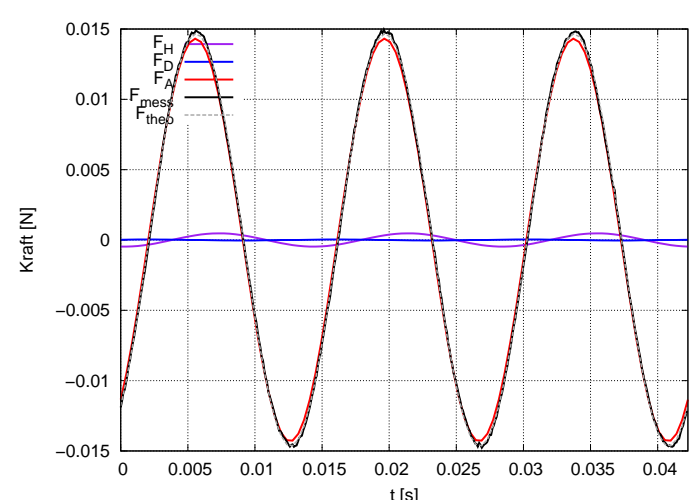

(c) $R=25 \mathrm{~mm}$

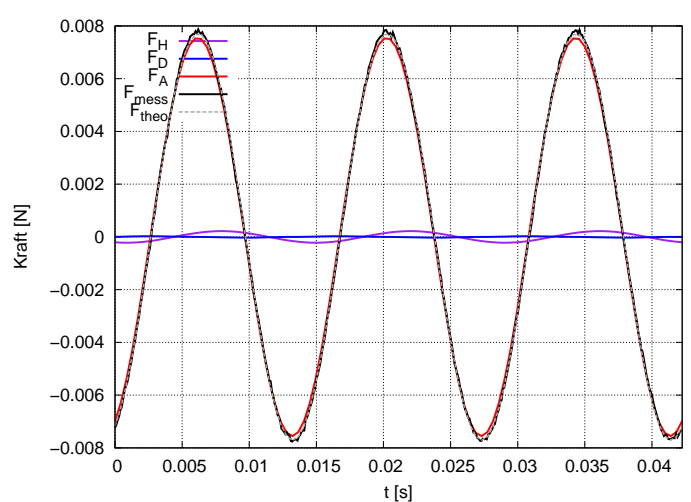

(b) $R=20 \mathrm{~mm}$

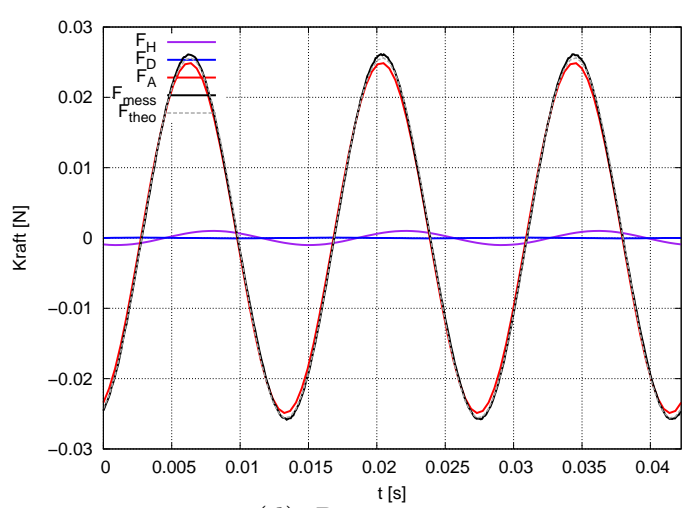

(d) $R=30 \mathrm{~mm}$

Abbildung C.2.: Kraftanteile im Vergleich mit der gemessenen Kraft für $f=71 \mathrm{~Hz}$. 


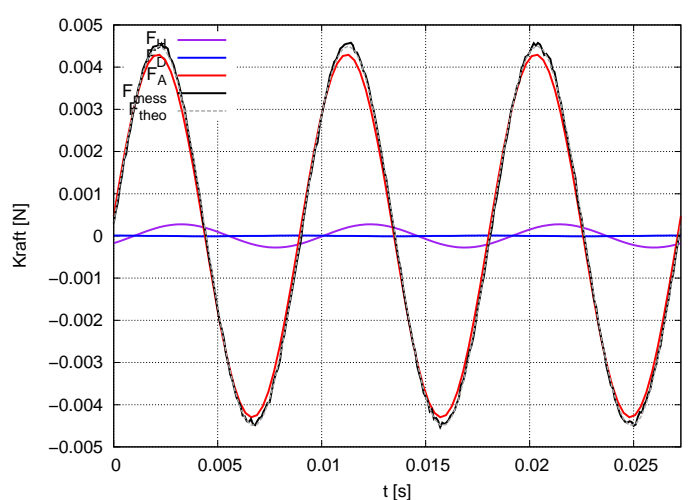

(a) $R=15 \mathrm{~mm}$

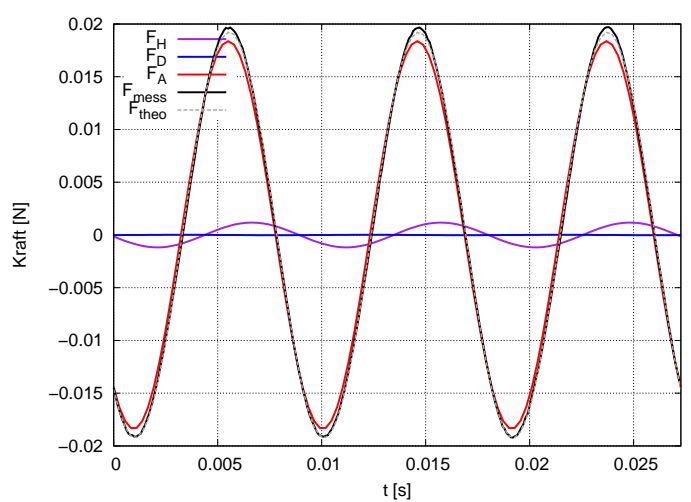

(c) $R=25 \mathrm{~mm}$

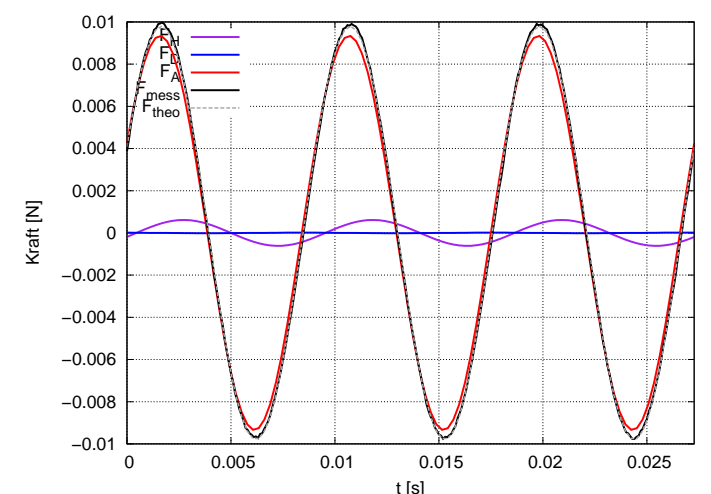

(b) $R=20 \mathrm{~mm}$

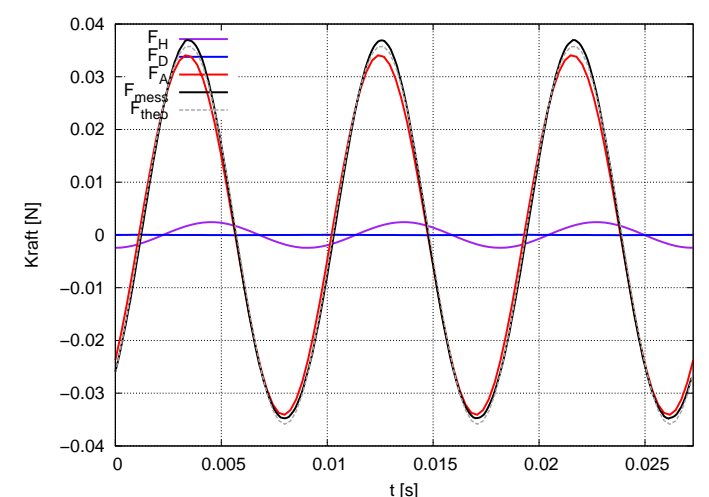

(d) $R=30 \mathrm{~mm}$

Abbildung C.3.: Kraftanteile im Vergleich mit der gemessenen Kraft für $f=110 \mathrm{~Hz}$. 


\section{Lautsprechermessungen}

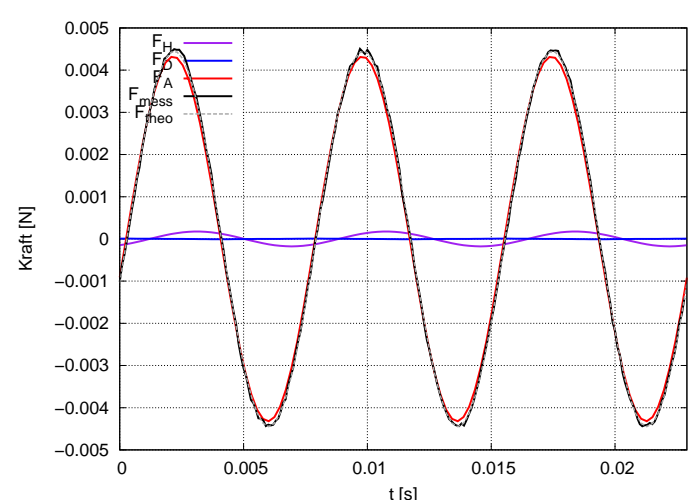

(a) $R=15 \mathrm{~mm}$

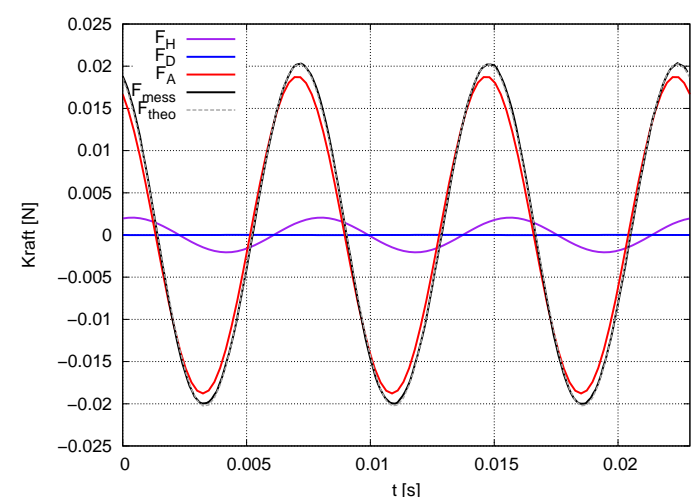

(c) $R=25 \mathrm{~mm}$

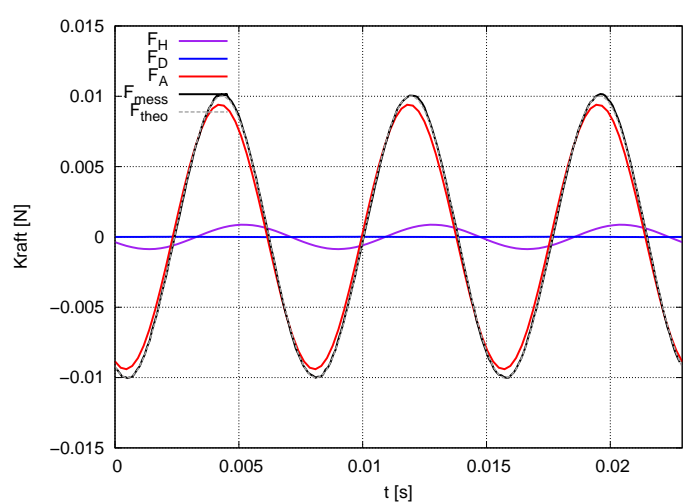

(b) $R=20 \mathrm{~mm}$

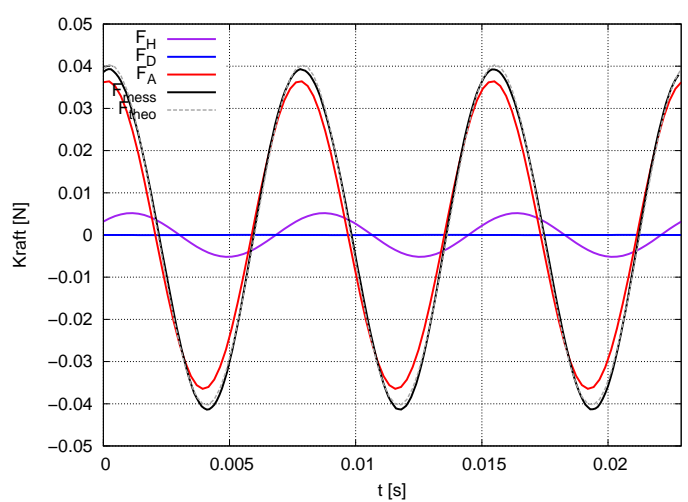

(d) $R=30 \mathrm{~mm}$

Abbildung C.4.: Kraftanteile im Vergleich mit der gemessenen Kraft für $f=110 \mathrm{~Hz}$. 


\begin{tabular}{|r|r|r|r|r|r|r|r|r|}
\hline $\mathrm{f}[\mathrm{Hz}]$ & Amp $[\mathrm{mV}]$ & $\mathrm{R}[\mathrm{mm}]$ & $A_{0}[\mathrm{~mm}]$ & $\mathrm{Re}$ & $\Delta F[\mathrm{mN}]$ & $A_{0} / 2 R$ & $A_{0} \omega[\mathrm{m} / \mathrm{s}]$ & $C_{A}^{*}$ \\
\hline \hline 30 & 200 & 15 & 0,743 & 278,59 & 0,77 & 0,025 & 0,140 & 2,267 \\
\hline 30 & 200 & 20 & 0,743 & 371,50 & 1,409 & 0,019 & 0,140 & 1,769 \\
\hline 30 & 200 & 25 & 0,743 & 464,38 & 3,452 & 0,015 & 0,140 & 2,214 \\
\hline 30 & 200 & 30 & 0,743 & 557,24 & 5,994 & 0,012 & 0,140 & 2,213 \\
\hline 30 & 1000 & 15 & 3,716 & 1393,03 & 3,944 & 0,124 & 0,700 & 2,343 \\
\hline 30 & 1000 & 20 & 3,716 & 1857,37 & 7,508 & 0,093 & 0,700 & 1,885 \\
\hline 30 & 1000 & 25 & 3,716 & 2321,87 & 18,021 & 0,074 & 0,700 & 2,317 \\
\hline 30 & 1000 & 30 & 3,716 & 2786,09 & 31,157 & 0,062 & 0,700 & 2,314 \\
\hline 40 & 500 & 15 & 1,465 & 732,17 & 2,65 & 0,049 & 0,368 & 2,249 \\
\hline 40 & 500 & 20 & 1,465 & 976,22 & 4,968 & 0,037 & 0,368 & 1,781 \\
\hline 40 & 500 & 25 & 1,465 & 1220,27 & 12,167 & 0,029 & 0,368 & 2,226 \\
\hline 40 & 500 & 30 & 1,465 & 1464,29 & 20,417 & 0,024 & 0,368 & 2,164 \\
\hline 40 & 1000 & 15 & 2,929 & 1464,43 & 5,396 & 0,098 & 0,736 & 2,302 \\
\hline 40 & 1000 & 20 & 2,929 & 1952,46 & 9,518 & 0,073 & 0,736 & 1,711 \\
\hline 40 & 1000 & 30 & 2,929 & 2928,68 & 42,951 & 0,049 & 0,736 & 2,288 \\
\hline 71 & 500 & 15 & 0,851 & 754,70 & 5,802 & 0,028 & 0,380 & 2,688 \\
\hline 71 & 500 & 20 & 0,851 & 1006,58 & 9,634 & 0,021 & 0,380 & 1,885 \\
\hline 71 & 500 & 25 & 0,851 & 1258,22 & 24,767 & 0,017 & 0,380 & 2,480 \\
\hline 71 & 500 & 30 & 0,851 & 1509,79 & 38,693 & 0,014 & 0,380 & 2,240 \\
\hline 71 & 500 & 15 & 0,851 & 754,93 & 5,729 & 0,028 & 0,380 & 2,655 \\
\hline 110 & 500 & 15 & 0,457 & 628,28 & 5,903 & 0,015 & 0,316 & 2,126 \\
\hline 110 & 500 & 20 & 0,457 & 837,75 & 11,933 & 0,011 & 0,316 & 1,812 \\
\hline 110 & 500 & 25 & 0,457 & 1047,17 & 29,159 & 0,009 & 0,316 & 2,268 \\
\hline 110 & 500 & 30 & 0,457 & 1256,62 & 47,572 & 0,008 & 0,316 & 2,141 \\
\hline 131 & 500 & 15 & 0,352 & 576,37 & 5,934 & 0,012 & 0,290 & 1,952 \\
\hline 131 & 500 & 20 & 0,352 & 768,54 & 11,154 & 0,009 & 0,290 & 1,551 \\
\hline 131 & 500 & 25 & 0,352 & 960,63 & 28,754 & 0,007 & 0,290 & 2,046 \\
\hline 131 & 500 & 30 & 0,352 & 1152,75 & 46,541 & 0,006 & 0,290 & 1,915 \\
\hline 200 & 500 & 20 & 0,142 & 473,71 & 17,441 & 0,004 & 0,179 & 2,576 \\
\hline 200 & 500 & 25 & 0,142 & 592,13 & 36,051 & 0,003 & 0,179 & 2,727 \\
\hline 200 & 500 & 30 & 0,142 & 710,58 & 56,46 & 0,002 & 0,179 & 2,480 \\
\hline 200 & 500 & 15 & 0,142 & 355,30 & 9,396 & 0,005 & 0,179 & 3,287 \\
\hline
\end{tabular}

Tabelle C.3.: Ergebnisse der Kraftmessung im instationären Lautsprecherfeld mit optischer Objektverfolgung. 



\section{E B E N S L A U F}

\section{PERSÖNLICHE ANGABEN}

Name: $\quad$ Sabrina Rutschmann

Staatsangehörigkeit: deutsch

Geburtsdatum: $\quad 18.02 .1987$

Geburtsort: Göttingen

\section{AUSBILDUNG UND STUDIUM}

$1999-2006$

Juni 2006

$2006-2011$

September 2009

September 2011

$2011-2017$
Felix-Klein-Gymnasium Göttingen

Abschluss Abitur, Note: 2,0

Studium der Physik an der Georg-August-Universität Göttingen

Abschluss Bachelor of Science (Physik), Note 2,3

Abschluss Master of Science (Physik), Note 1,4 mit Auszeichnung

Promotionsstudium in Physik an der Georg-AugustUniversität Göttingen

\section{BERUFSERFAHRUNG}

$2009-2011$

$2011-2017$
Studentische Hilfskraft am Deutschen Zentrum für Luftund Raumfahrt, Göttingen

Doktorandin und wissenschaftliche Mitarbeiterin am Deutschen Zentrum für Luft- und Raumfahrt, Göttingen 
2012

2013

2013

2013

2014
18. DGLR Fachsymposium der STAB, Stuttgart

International Workshop on Train Aerodynamics, Birmingham, Großbritannien

Rail Aerodynamics 2013, Berlin

WCRR 2013, Sydney, Australien

Railways 2014, Korsika, Frankreich

\section{VERÖFFENTLICHUNGEN}

2013

2013

2014

2014
„A flexible potential flow model for the prediction of aerodynamic forces on track side objects" in: Proceedings of the 10th World Congress on Railway Research (WCRR)

"Validation of a potential flow model for the prediction of the aerodynamic lods on track side objects“" in: Aerodynamics of Trains and Infrastructure, 44/201. Verlag des IVF Bahntechnik e.V. ISBN 978-3-940727-37-4

„Aerodynamic Loads induced by Passing Trains on Track Side Objects" in: New Results in Numerical and Exprimental Fluid Mechanics IX, Springer International Publishing. ISBN 978-3-319-03157-6

„Simplified model to estimate forces on track side objects" in: Proceedings of the Second International Conference on Railway Technology: Research, Development and Maintenance, ISBN: 978-1-905088-59-1 